Aus der Abteilung Anatomie und Embryologie

(Prof. Dr. med. C. Viebahn)

im Zentrum Anatomie

der Medizinischen Fakultät der Universität Göttingen

\title{
Paläopathologische Untersuchungen am Cranium der Population von Baunach (Oberfranken) mit besonderer Berücksichtigung der spezifischen und unspezifischen Infektionskrankheiten
}

Ein Beitrag zur Rekonstruktion des Gesundheitsstatus einer frühmittelalterlichen dörflichen Population Süddeutschlands

\author{
Inaugural-Dissertation \\ zur Erlangung des Doktorgrades \\ der Medizinischen Fakultät \\ der Georg-August-Universität zu Göttingen
}

vorgelegt von

Christian Schulz

aus

Sondershausen

Göttingen 2011 
Dekan: Prof. Dr. med. C. Frömmel

I. Berichterstatter: Prof. Dr. med. Dr. phil. M. Schultz

II. Berichterstatter/in:

III. Berichterstatter/in:

Tag der mündlichen Prüfung: 


\section{Inhaltsverzeichnis}

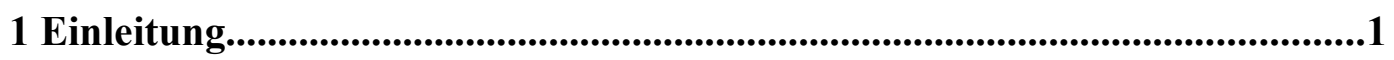

1.1 Historischer Hintergrund des Baunacher Gräberfelds..................................1

1.2 Aufgabe der Arbeit.............................................................................2

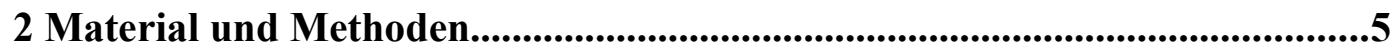

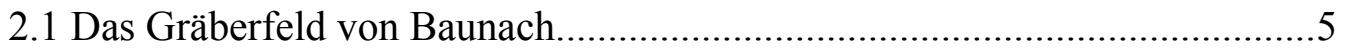

2.2 Einteilung des Alters und Geschlechts........................................................5

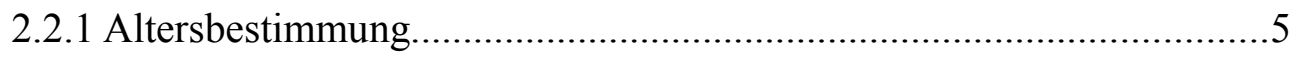

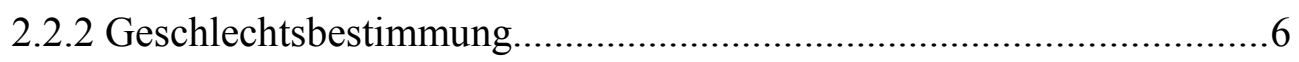

2.3 Paläopathologische Bearbeitung der Skeletfunde........................................ 7

2.4 Katalogbeschreibung...................................................................... 10

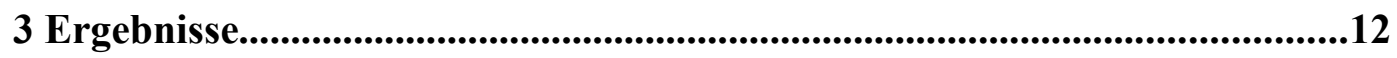

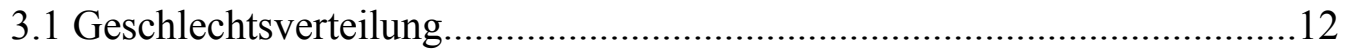

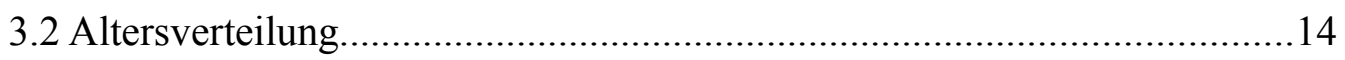

3.2.1 Altersverteilung der Männer............................................................16

3.2.2 Altersverteilung der Frauen............................................................... 18

3.2.3 Altersverteilung nicht bestimmbarer Individuen..............................21

3.3 Pathologische Befunde in der Nasenhöhle..............................................23

3.3.1 Anatomische und physiologische Grundlagen...................................23

3.3.2 Erkrankungen in der Nasenhöhle......................................................24

3.3.3 Ergebnisse Nasenhöhle.....................................................................26

3.4 Pathologische Befunde in den Nasennebenhöhlen...................................29

3.4.1 Anatomische und physiologische Grundlagen..................................29

3.4.2 Erkrankungen der Nasennebenhöhlen..............................................31

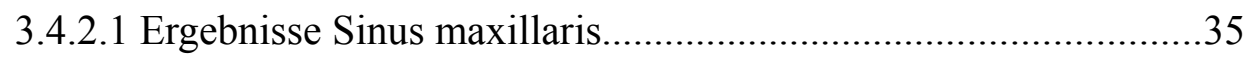

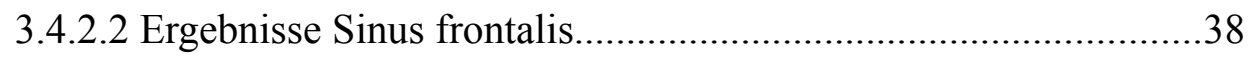

3.5 Pathologische Befunde im Mittelohr .......................................................42

3.5.1 Anatomische und physiologische Grundlagen..................................42

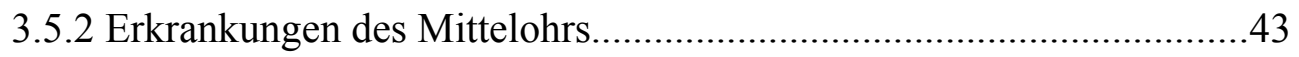

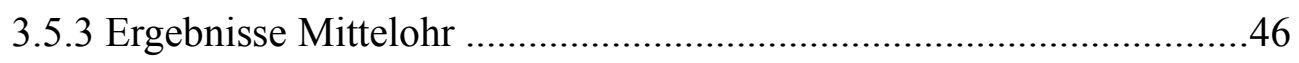

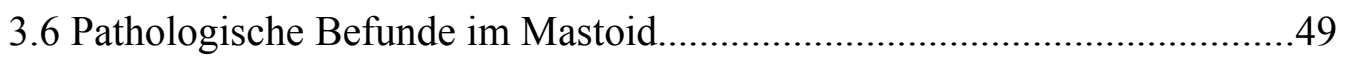

3.6.1 Anatomische und physiologische Grundlagen.................................49 


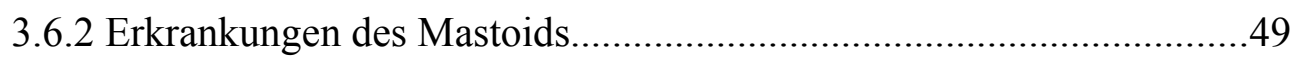

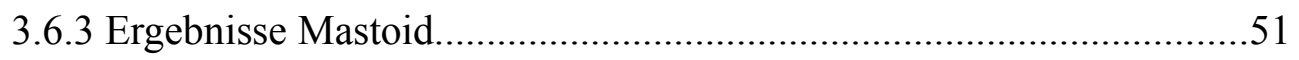

3.7 Pathologische Befunde im Schädelinnenraum........................................58

3.7.1 Anatomische und physiologische Grundlagen....................................58

3.7.2 Erkrankungen des Schädelinnenraumes..........................................60

3.7.2.1 Tuberkulöse Meningitis..........................................................61

3.7.3 Ergebnisse Schädelinnenraum ....................................................63

3.8 Pathologische Befunde am Schädeldach ................................................6 69

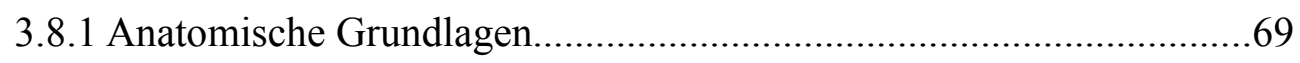

3.8.2 Erkrankungen des Schädeldachs......................................................69

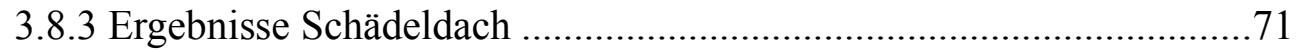

3.9 Pathologische Befunde in den Sulci der venösen Hirnblutleiter..................75

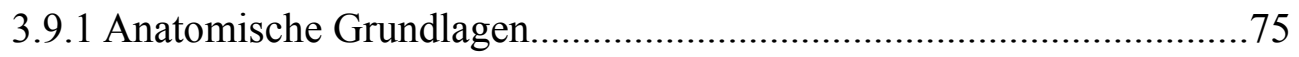

3.9.2 Erkrankungen der venösen Hirnblutleiter...........................................76

3.9.2.1 Ergebnisse Sinus sagittalis superior....................................... 78

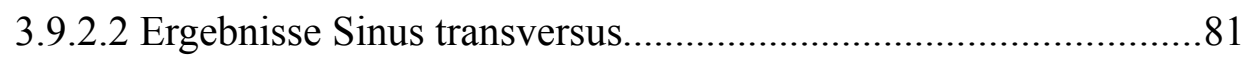

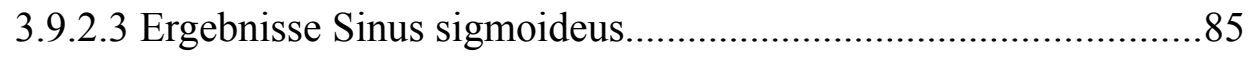

3.10 Pathologische Befunde in der Orbita......................................................89

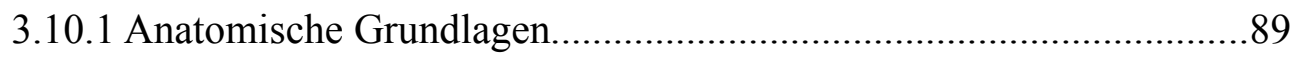

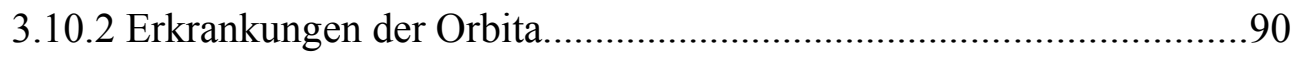

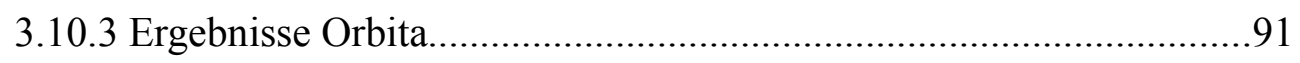

3.11 Pathologische Befunde am harten Gaumen...........................................96

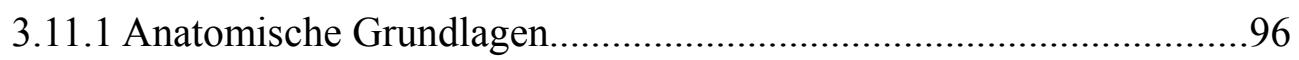

3.11.2 Erkrankungen des harten Gaumens.............................................96

3.11.3 Ergebnisse harter Gaumen.........................................................98

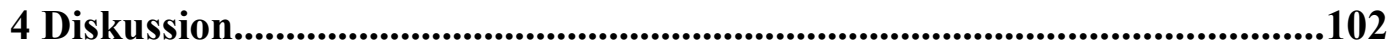

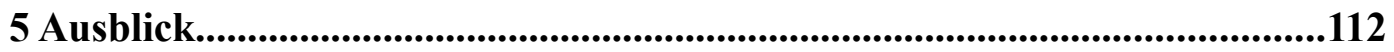

6 Zusammenfassung................................................................................16

7 Anhang: Bild 1 - 30................................................................................................119

8 Literaturverzeichnis...........................................................................................163

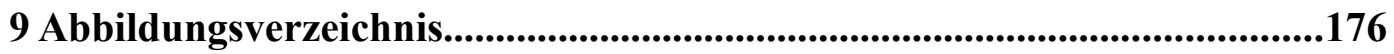

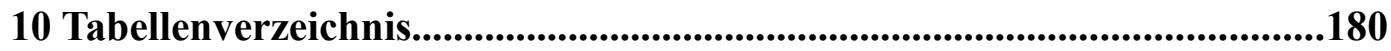




\section{Einleitung}

\subsection{Historischer Hintergrund des Baunacher Gräberfelds}

Die heute etwa 4000 Einwohner zählende Stadt Baunach liegt im Landkreis Bamberg im Tal des Flüsschens Baunach und nahe dessen Mündung in den Main. Der Landkreis Bamberg befindet sich in Oberfranken im Norden Bayerns. Eine lang bestehende Besiedelung dieser Landschaft wird durch zahlreiche Bodenfunde belegt und reicht bis in die Steinzeit (WALTER 2002). Die erstmalige schriftliche Erwähnung einer Stadt an diesem Ort findet sich als frühmittelalterliches "Bunahu" im Rahmen einer Schenkungsurkunde an das Kloster Fulda im Jahre 802. Eine Kirche wird in Bunahu um 816 angegeben, wobei für 824 eine Kirchenweihung in Bunahu belegt ist. Im Rahmen einer Neubebauung des Marktplatzes in Baunach wurde 1998 eine Grabung notwendig. Bauarbeiten stießen auf einen unzerstörten frühmittelalterlichen Bestattungshorizont neben der Kirche St. Oswald. Durch die Außenstelle Oberfranken des bayerischen Landesamtes für Denkmalpflege und die Ausgrabungsfirma ReVe, Büro für Archäologie (Bamberg), wurde ein 89 Gräberfelder umfassender Friedhofshorizont freigelegt. Die Gräber befanden sich in unmittelbarer Nähe einer Kirche, deren Weihung für das Jahr 824 schriftlich belegt ist. Aus diesem Datum und den wenigen gefundenen Grabbeigaben aus den älteren Gräbern kann man eine Belegung des Friedhofes von ca. 820 bis ungefähr 1000 annehmen. Mehrheitlich fanden sich frühmittelalterliche beigabenlose Körpergräber, wobei sich die wenigen Grabbeigaben auf weibliche Ohr- und Fingerringe sowie Trachtbestandteile beschränkten. Das Fundspektrum umfasste in erster Linie Frauentracht aus dem 8. und 9. Jahrhundert. Überwiegend konnten zur genaueren chronologischen Einordnung für die Karolingerzeit typische Schmuckformen geborgen werden. Es fanden sich auch vereinzelte spätmerowingische Schmuckformen. Alle Toten wurden in gestreckter Rückenlage mit dicht anliegenden Armen in einfachen Erdgräbern beigesetzt. Grabüberschneidungen und Mehrfachbelegungen kamen nahe der Kirche häufiger vor. Einige der Größe nach vermutlich als Kindergräber einzuordnende Grabstätten zeigten sich leer (HENSCH 1998). 
Der Erhaltungszustand der vorgefundenen Skelete reicht von fast komplett vorhandenen Individuen bis hin zu sehr stark fragmentarisch erhaltenen Skeletfunden. Dabei überwiegen fragmentarisch erhaltene Skelete mit partiell stark erodierter Oberfläche.

\subsection{Aufgabe der Arbeit}

Durch archäologische Untersuchungen sind viele Lebensumstände der Menschen aus vergangener Zeit bekannt. Jedoch vermitteln diese nur eine indirekte Aussage über die Auswirkung des Alltags auf das einzelne Individuum. Genauere Aussagen über individuelle Lebensumstände und deren Auswirkungen können durch rein archäologische Forschungsmethoden nicht formuliert werden. Häufig liegen auch keine weiteren Überlieferungen vor, so dass nur Vermutungen und Spekulationen über Leben und Sterben unserer Vorfahren getroffen werden können.

Mit Hilfe paläopathologischer Untersuchungen an geeignetem Skeletmaterial lassen sich Erkrankungshäufigkeiten und -verteilungsmuster beweisen. Auch epidemiologische Untersuchungen sowie Aussagen über auffällige Unterschiede in der sozialen Struktur können daraus entstehen. Werden diese paläopathologischen Befunde in historische Forschungsergebnisse eingebunden, erhöht sich die Aussagekraft historischer Funde. Menschliche Hintergründe geschichtlicher Ereignisse werden deutlich und ein differenzierter Blick auf archäologisch gewonnene historische Funde ist möglich.

Mit Hilfe paläopathologischer Aufarbeitung der bei archäologischen Grabungen freigelegten Skeletfunde können für das einzelne Individuum bezüglich Auswirkungen und Umstände des Lebensalltags genauere Aussagen getroffen werden (SCHULTZ 1982). Die Paläopathologie wird somit als das Studium der Krankheiten in der Vorgeschichte aus historischen, diagnostischen und epidemiologischen Perspektiven beschrieben, welche verschiedenste Aspekte der sozialen und physischen Umwelt untersuchter Individuen erklären kann (LARSEN 2003, ORTNER 2003, WELLS 1967). 
Da sich Spuren verschiedener Erkrankungen an Skeleten auch noch nach Jahrhunderten erhalten haben, können so mit Hilfe paläopathologischer Untersuchungen die archäologischen Ergebnisse ergänzt werden. Unser Bild von der Geschichte kann somit unabhängig von schriftlichen Überlieferungen umfassender ausfallen (DASTUGUE 1978).

In den nachfolgenden Kapiteln sollen die Spuren verschiedener Krankheiten sowie mögliche Krankheitsauswirkungen auf das alltägliche Leben und die Gesellschaftsstruktur erörtert werden. Durch das Wissen um bestehende Erkrankungen, verbunden mit der archäologisch nachgewiesen Umgebungssituation, wird ein Verständnis der alltäglichen Lebensumstände bei den frühmittelalterlichen Skeletfunden nach paläopathologischer Untersuchung umfassender.

Bestimmte Erkrankungen können für viele Lebensumstände einer frühmittelalterlichen Population charakteristisch sein. Die Verteilungsmuster der in dieser Untersuchung nachgewiesenen Erkrankungen vermitteln hinsichtlich der dörflichen Lebensgemeinschaft des frühmittelalterlichen Baunach wichtige Hinweise auf damalige Lebensumstände (PADBERG 1992). Durch eventuell bei den Geschlechtern zu erwartende Unterschiede ist eine gewisse soziologische Betrachtung des damaligen Zusammenlebens möglich. Auch die Bedeutung von Kindern im Mittelalter kann durch Auswertung der vorkommenden Krankheiten und Verteilungsmuster näher beleuchtet werden.

Der Schwerpunkt dieser paläopathologischen Untersuchung liegt auf der Ätiologie und Epidemiologie verschiedener Erkrankungen. Dabei beziehen sich die in dieser Arbeit vorgelegten Ergebnisse ausschließlich auf die am Cranium der Baunacher Individuen erhobenen Befunde.

Ein Vergleich mit Daten, welche an anderen, etwa zeitgleich lebenden Populationen der Karolingerzeit erhoben wurden, beleuchtet die Lebensumstände einer Zeit, in der es noch keine flächendeckende medizinische Versorgung mit Antibiotika und modernen Therapiekonzepten gab. Da dieser Zustand jedoch auch in unserer heutigen Zeit für viele Menschen in Krisengebieten und Entwicklungsländern Normalität ist, können durch die paläopathologische Forschung auch aktuell verwertbare Erkenntnisse gewonnen werden. Es zeigen sich bei unbehandelt ver- 
laufenden Erkrankungen häufig Erkrankungsschweregrade, welche in der heutigen Zeit mit modernen Therapiekonzepten und adäquater medizinischer Versorgungsstruktur nicht mehr auftreten.

Die paläopathologische Untersuchung kann helfen, das Verständnis für die Bedeutung einer Erkrankung des einzelnen Individuums und auch für den Verbund einer dörflichen Lebensgemeinschaft im historischen Kontext zu vergrößern.

Da verschiedene Erkrankungen und Erkrankungsverteilungen einen Indikator für den Sozialzustand einer Population darstellen, sind die paläopathologischen Ergebnisse im Vergleich mit aktuell gewonnenen Daten zudem ein Instrument zur Darstellung der Entwicklung unterschiedlicher Erkrankungen und Zivilisationsprozesse. 


\section{Material und Methoden}

\subsection{Das Gräberfeld von Baunach}

Die Skelete wurden von der Außenstelle Bamberg des Bayerischen Landesamtes für Denkmalpflege zur weiteren paläopathologischen Bearbeitung nach Göttingen ausgeliehen. Hier erfolgte im Zentrum Anatomie Göttingen, Abteilung Morphologie, Sektion Paläopathologie eine Auswertung der Funde, wobei sich diese Arbeit auf das Cranium beschränkt und sich ausschließlich mit den Krankheiten dieser Region beschäftigt. Soweit durch die Grabung geborgen liegt jedoch auch das Postcranium der Skelete zur weiteren Untersuchung vor.

Es kam häufig bei Mehrfachbelegungen und Grabüberschneidungen zur Vermischung der Skelete mehrerer Individuen, welche bei der paläopathologischen Untersuchung initial durch Auslegung der vorhandenen Langknochen und Schädel einem Individuum zugeordnet werden mussten.

Insgesamt sind 87 Individuen vorhanden, von denen jedoch nur 52 Individuen (59,8 Prozent) mit mindestens einem Fragment des Schädels repräsentiert sind.

Die für diese Untersuchungen in die Arbeit bearbeiteten Individuen sind der Tabelle 2 zu entnehmen.

\subsection{Einteilung des Alters und Geschlechts}

\subsubsection{Altersbestimmung}

Die Altersbestimmung der Individuen erfolgte überwiegend nach FEREMBACH et al. (1979) sowie teilweise nach SZILVÁSSY (1988). Bis auf sieben Individuen konnten alle Skelete bestimmt werden. Diese sieben Skelete waren nach diesen Verfahren bei zu fragmentarischem und erodiertem Erhaltungszustand nicht bestimmbar oder wiesen als Kinderskelete noch keine geschlechtsspezifischen Merkmale auf. 
Die ermittelten Lebensjahre lagen in einer Spanne von 0 bis maximal 75 Jahren. Zur Vereinfachung der Ergebnisdarstellung erfolgte eine Unterteilung der Lebensalter in neun Klassen (Tab. 1) :

\begin{tabular}{|c|c|c|}
\hline $0-1,9$ & Lebensjahre & Infans Ia \\
\hline $2-5,9$ & Lebensjahre & Infans Ib \\
\hline $6-13,9$ & Lebensjahre & Infans II \\
\hline $14-19,9$ & Lebensjahre & Juvenil \\
\hline $20-29,9$ & Lebensjahre & Frühadult \\
\hline $30-39,9$ & Lebensjahre & Spätadult \\
\hline $40-49,9$ & Lebensjahre & Frühmatur \\
\hline $50-59,9$ & Lebensjahre & Spätmatur \\
\hline $60+$ & Lebensjahre & Senil \\
\hline
\end{tabular}

Tabelle 1: Lebensalter Klasseneinteilung

Mit Hilfe dieser Einteilung wurde die demographische Verteilung mit Hilfe eines computergestützten Rechenprogramms, welches Frau Dr. Kosak, Anthropologische Abteilung des Archäologischen Institutes der Ukrainischen Akademie der Wissenschaften zu Kiew, dem Bearbeiter zur Verfügung gestellt hatte, berechnet.

\subsubsection{Geschlechtsbestimmung}

Die anthropologische Geschlechtszuordnung erfolgte überwiegend nach FEREMBACH et al. (1979) und SJVØLD (1988). Das Postcranium (sofern vorhanden) wurde dazu - wie auch schon bei der Altersbestimmung - mit benutzt.

Bei nicht eindeutig zu bestimmenden Individuen erfolgte eine Einteilung in Untergruppen. 
Daraus resultieren folgende Geschlechtszugehörigkeiten:

1. M: männliches Individuum

2. $\mathrm{M} \gg \mathrm{W}$ : sehr wahrscheinlich männliches Individuum

3. $\mathrm{M}>\mathrm{W}$ : wahrscheinlich männliches Individuum

4. $\quad \mathrm{M}>=\mathrm{W}$ : eher männlich als weibliches Individuum

5. W: weibliches Individuum

6. $\mathrm{W}>>\mathrm{M}$ : sehr wahrscheinlich weibliches Individuum

7. W $>$ M: wahrscheinlich weibliches Individuum

8. $\mathrm{W}>=\mathrm{M}$ : eher weibliches als männliches Individuum

9. $\mathrm{M}=\mathrm{W}$ : Individuum weist Merkmale beider Geschlechter auf (unbestimmbar)

Individuen aus den Kategorien eins bis vier werden als männliche Individuen gewertet.

Als weibliche Individuen gelten Vertreter der Kategorien fünf bis acht.

In der neunten Kategorie finden sich Skelete, an denen das knöcherne Material keine Zuordnung des Geschlechts zulässt beziehungsweise keine überwiegende Tendenz der geschlechtsspezifischen Merkmale erkennbar ist.

\subsection{Paläopathologische Bearbeitung der Skeletfunde}

Die vorhandenen Schädelknochen wurden zunächst, sofern dies für eine genaue Befundung noch nicht ausreichend geschehen war, von Erde gereinigt und in anatomisch-topographischem Zusammenhang ausgelegt. Diese Reinigung erfolgte ausschließlich mit Wasser und Pinsel oder trocken mit einer Bürste. Das zum Teil sehr stark fragmentierte Material wurde nach Möglichkeit - zur Erleichterung der Zuordnung und Dokumentation - zu größeren Fragmenten mit Holzkaltleim 
(PONAL®) zusammengesetzt. Der Erhaltungszustand der Knochen und die Repräsentation des Craniums wurden schriftlich in speziellen Formblättern dokumentiert.

Zunächst erfolgte eine achtfach vergrößernde lupenmikroskopische Untersuchung der gesäuberten Knochen. Bei fraglichen oder pathologisch erscheinenden Befunden konnten weiterführende Untersuchungen wie Lichtmikroskopie, Rasterelektronenmikroskopie, Endoskopie oder Radiologie herangezogen werden, um ein Höchstmaß an diagnostischer Aussagekraft und Genauigkeit zu erreichen.

Knochenproben für die Knochendünnschliffmikroskopie und Rasterelektronenmikroskopie wurden an repräsentativen Stellen entnommen.

Für lichtmikroskopische Untersuchungen wurden die Knochenproben zu zwei Dünnschliffen in den Stärken von 50 bzw. $70 \mu \mathrm{m}$ (SCHULTZ und DROMMER 1983; Methode von SCHULTZ , in: Knussmann 1988a, 1988b;) verarbeitet und anschließend unter einfachem und polarisiertem Durchlicht mit einem Polarisationsmikroskop der Firma LEICA ${ }^{\circledR}$ untersucht. Die Befunde wurden fotografisch dokumentiert.

Die für die Rasterelektronenmikroskopie bestimmten Proben wurden zunächst mit einer Gold-Palladiumschicht bedampft und anschließend mit dem Rasterelektronenmikroskop DPM 960 der Firma Zeiss ${ }^{\circledR}$ befundet.

Alle für die mikrokopische Untersuchung aufgearbeiteten Knochen sowie sämtliche anderen Krankheitsspuren wurden vorher zu Dokumentations- und Vergleichszwecken digital fotografiert (Toshiba ${ }^{\circledR}$ PDR-M25, Pentax ${ }^{\circledR}$ Optio RS $330)$.

Die radiologische Untersuchung von fraglichen oder pathologisch interessanten Befunden erfolgte mit Hilfe des Geräts „Faxitron“ der Firma Hewlett \& Packard ${ }^{\circ}$. Für die Aufnahmen wurde der folienlose Röntgenfilm „Cronex NDT 65 “ der Firma Du Pont ${ }^{\circledR}$ verwendet.

Die folgende Tabelle 2 listet die für diese Arbeit verwendeten 52 Baunacher Individuen mit Schädel auf. 


\begin{tabular}{|c|c|c|c|}
\hline Individuumbezeichnung & Grabnummer & Geschlecht & Lebensalter \\
\hline Bau 1 & 1 & weiblich & (55) $60-65(69)$ \\
\hline Bau 2 & 2 & männlich = weiblich & (5) $6-7(8)$ \\
\hline Bau 3 & 3 & männlich & (50) $55-59(65)$ \\
\hline Bau 4 & 4 & männlich & \\
\hline Bau 5 & 5 & weiblich & (9) $12-12(16)$ \\
\hline Bau 6 & 6 & männlich & (30) $35-49(55)$ \\
\hline Bau 8 (= Bau 15) & 8 & männlich & $24-29$ \\
\hline Bau 9 & 9 & männlich & (55) $60-69(75)$ \\
\hline Bau 11 & 10 & männlich $>=$ weiblich & $30-39(49)$ \\
\hline Bau 16 & 15 & männlich = weiblich & $>25$ \\
\hline Bau 17 & 14 & männlich & $60-65(69)$ \\
\hline Bau 17a & 14 & männlich & $35-49$ \\
\hline Bau 19 & 12 & männlich & (45) $50-55(59)$ \\
\hline Bau 20 & 20 & weiblich $>=$ männlich & (15) $17-19(20)$ \\
\hline Bau 21 & 21 & weiblich & $25-29$ \\
\hline Bau 30 & 28 & männlich & (25) $30-39$ \\
\hline Bau 31 & 29 & männlich = weiblich & (24) $25-30$ \\
\hline Bau 33 & 30 & weiblich & (30) $35-45$ \\
\hline Bau 35-1 & 32 & weiblich > männlich & $20-25$ \\
\hline Bau 35-1a & 34 & männlich = weiblich & (7) $8-10(11)$ \\
\hline Bau 35-2-1 & 34 & männlich & (50) $55-59(65)$ \\
\hline Bau 35-2-2 & 32 & weiblich > männlich & $15-20$ \\
\hline Bau 36 & 33 & männlich & $25-29(30)$ \\
\hline Bau 37 & 34 & männlich $=$ weiblich & (6) $7-9(10)$ \\
\hline Bau 39 & 36 & männlich & $60-65(69)$ \\
\hline Bau 40 & 40 & männlich & (24) $25-30(35)$ \\
\hline Bau 41 & 37 & männlich = weiblich & $1,5-2$ \\
\hline Bau 47 & 41 & männlich & $21-25(30)$ \\
\hline Bau 49 & 43 & männlich & $35-55$ \\
\hline Bau 52 & 44 & männlich = weiblich & $25-45(49)$ \\
\hline Bau 55 & 47 & weiblich & (22) $25-30$ \\
\hline Bau 59 & 48 & männlich = weiblich & ca. 10. Monat \\
\hline Bau 61 & 51 & männlich & $60-65(69)$ \\
\hline Bau 63 & 53 & männlich >= weiblich & (35) $40-50(55)$ \\
\hline Bau 65 & 55 & weiblich & (25) $30-39(45)$ \\
\hline Bau 66 & 56 & weiblich $>=$ männlich & $45-55$ \\
\hline Bau 67 & 57 & männlich & $40-49$ \\
\hline Bau 68 & 58 & männlich & (18) $20-21(25)$ \\
\hline Bau 69 & 59 & männlich & $20-21(24)$ \\
\hline Bau 74 & 64 & weiblich > männlich & (3) $3,5-4(4,5)$ \\
\hline Bau 79 & 69 & männlich & (45) $50-55(59)$ \\
\hline Bau 80 & 70 & männlich & (50) $55-65$ \\
\hline Bau 82 & 71 & männlich = weiblich & (4) $4,5-5,5(6)$ \\
\hline Bau 86 & 75 & männlich & $30-59(65)$ \\
\hline Bau 87 & 76 & männlich = weiblich & $45-55$ \\
\hline Bau 88 & 77 & weiblich > männlich & (45) $50-55(59)$ \\
\hline Bau 89 & 78 & männlich $\gg$ weiblich & $55-65$ \\
\hline Bau 93 & 82 & männlich $\gg$ weiblich & (20) $23-25$ \\
\hline Bau 94 & 83 & männlich > weiblich & $25-39(45)$ \\
\hline Bau 96 & 85 & männlich & $(50) 55-60(65)$ \\
\hline Bau 98 & 86 & weiblich & $22-25$ \\
\hline Bau 106 & 88 & weiblich $\gg$ männlich & $25-29(30)$ \\
\hline
\end{tabular}

Tabelle 2: Individuen Baunach mit Cranium 


\subsection{Katalogbeschreibung}

Die bei der paläopathologischen Untersuchung gewonnenen Befunde wurden in ein standardisiertes Schädel-Untersuchungsformular zur Dokumentation der Befunde (nach SCHULTZ 1986, 1994) eingetragen (Abb. 1).

\begin{tabular}{|c|c|c|c|}
\hline Signatur : & & Grab-Nr.: & $\begin{array}{l}\text { Archäologische } \\
\text { Bemerkung: }\end{array}$ \\
\hline Alter : & & Geschlecht: & \\
\hline \multicolumn{4}{|c|}{ Makroskopischer Befund } \\
\hline Lamina externa & re & & i \\
\hline Lamina interna & re & & i \\
\hline Fossa cranii ant. & re & & i \\
\hline Fossa cranii med. & re & & $\mathrm{i}$ \\
\hline Fossa cranii post. & re & & i \\
\hline \multicolumn{4}{|l|}{ Sella } \\
\hline \multicolumn{4}{|l|}{ Clivus } \\
\hline \multicolumn{4}{|l|}{ Sulc.sin.sag.sup. } \\
\hline Sulc.sin.transversi & re & & $\mathrm{i}$ \\
\hline Sulc.sin.sigmoidei & re & & i \\
\hline Orbitadach & re & & i \\
\hline Orbitaboden & re & & $\mathrm{i}$ \\
\hline $\begin{array}{l}\text { Ductus } \\
\text { nasolacrimalis }\end{array}$ & re & & i \\
\hline Sinus frontalis & re & & $\mathrm{i}$ \\
\hline Sinus maxillaris & re & & $\mathrm{i}$ \\
\hline Sinus sphenoidalis & re & & $\mathrm{i}$ \\
\hline Cell. ethmoidales & re & & $\mathrm{i}$ \\
\hline Nasenhöhle & re & & $\mathrm{i}$ \\
\hline Jochbeinaußenfl. & re & & i \\
\hline Begr. Apert. pirif. & re & & $\mathrm{i}$ \\
\hline Mittelohr & re & & i \\
\hline Meatus acust. ext. & re & & i \\
\hline Meatus acust. int. & re & & i \\
\hline Proc. Mastoideus & re & & i \\
\hline
\end{tabular}

Abbildung 1: Formblatt zur pathologischen Auswertung des Cranium 
Zusätzlich erfolgte eine grafische Darstellung der jeweiligen pathologischen Befunde in einem gesonderten Formblatt, welches die Erhaltung sowie die Repräsentanz der Lamina interna des gesamten Schädels und der Lamina externa des Schädeldaches dokumentiert.

Anschließend wurden die Untersuchungsergebnisse mit Windows ${ }^{\circledR}$ Excel in statistisch auswertbare Tabellen umgewandelt. 


\section{Ergebnisse}

\subsection{Geschlechtsverteilung}

Von den vorhandenen 87 Individuen waren 52 durch Schädel oder Schädelfragmente repräsentiert. Somit lagen für die Untersuchung 59,8 Prozent der Gesamtindividuenzahl vor.

Insgesamt zeigte sich folgende Aufteilung der Geschlechter der anthropologisch bestimmbaren Individuen (Abb. 2):

Es konnten 41 Männer, zu denen auch Skelete mit den Merkmalen männlich >= weiblich, männlich $>$ weiblich und männlich $>>$ weiblich identifiziert werden. Diese Gruppe entspricht 47 Prozent der Gesamtindividuen.

Für die 27 vorhandenen Frauen ergibt sich der entsprechende Wert von 31 Prozent. Auch hier zählten Individuen mit den Geschlechtsmerkmalen weiblich $>=$ männlich, weiblich $>$ männlich und weiblich $>>$ männlich als Frauen.

Für die nicht differenzierbaren Individuen, also männlich = weiblich, ergibt sich bei 19 vorhanden Individuen mit diesen Merkmalen eine Häufigkeit von 22 Prozent.

Weibliche Individuen machen etwa ein Drittel (31 Prozent) der Gesamtindividuen aus. Skelete mit nicht bestimmbarem Geschlecht sind zu etwa einem Fünftel ( 22 Prozent ) zu finden. 


\section{Geschlechtsverteilung der Baunacher \\ Gesamtpopulation $(n=87)$}

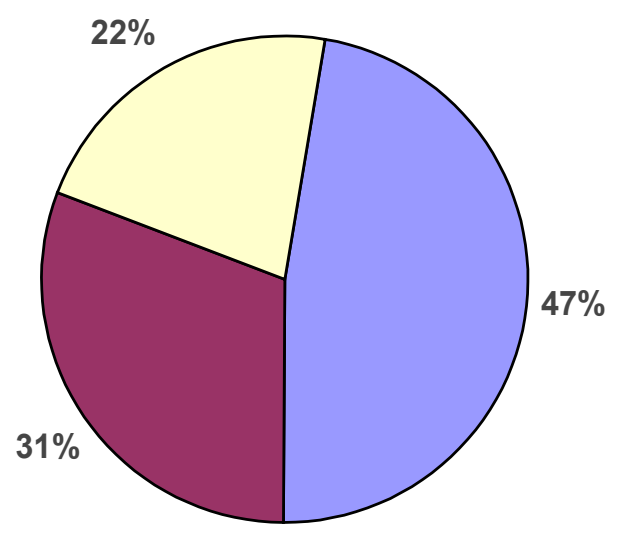

口 Männer ( $n=41)$

$\square$ Frauen $(\mathrm{n}=27)$

$\square$ Mann = Frau $(n=19)$

Abbildung 2: Geschlechtsverteilung in der gesamten Population von Baunach (7. - 9. Jhd.)

Die Verteilung der Geschlechter der in dieser Arbeit behandelten 52 Individuen ist Abbildung 3 zu entnehmen:

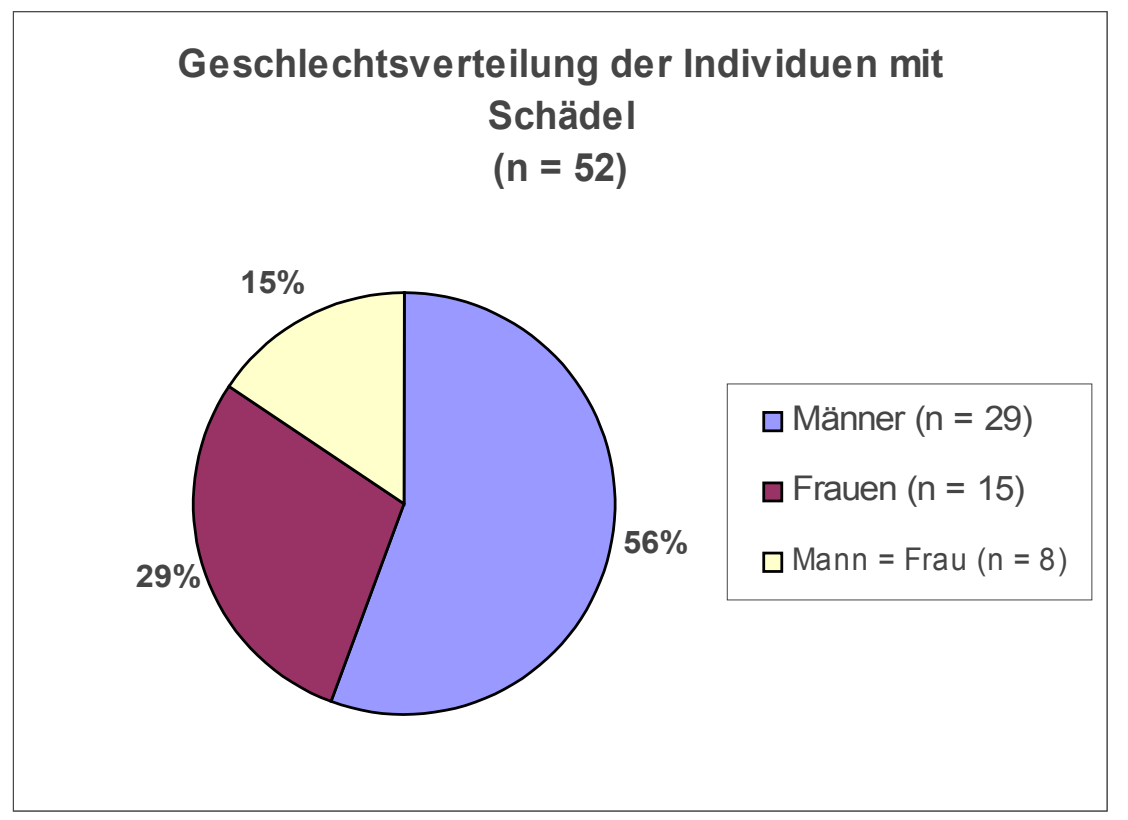

Abbildung 3: Geschlechtsverteilung der Baunacher Individuen mit erhaltenem Schädel (7. - 9. Jhd.) 
Das Diagramm stellt die prozentuale Verteilung der für die Arbeit untersuchten 29 Männer, 15 Frauen und 8 nicht bestimmbare Individuen dar. Es wurden wiederum Skelete mit männlich $>=$ weiblich, männlich $>$ weiblich männlich als Männer und weiblich $>=$ männlich, weiblich $>$ männlich sowie weiblich als Frauen gezählt. Das Überwiegen männlicher Individuen ist hier noch stärker als in der Gesamtpopulation ausgeprägt.

\subsection{Altersverteilung}

Die durch Bewertung von Individualmerkmalen am Skelet ( Cranium mit Zähnen sowie Postcranium ) ermittelten Sterbealter der Individuen ist der Tabelle $2 \mathrm{zu}$ entnehmen. Die statistische Auswertung zeigt für die Individuen mit Schädel folgende Ergebnisse (Abb. 4):

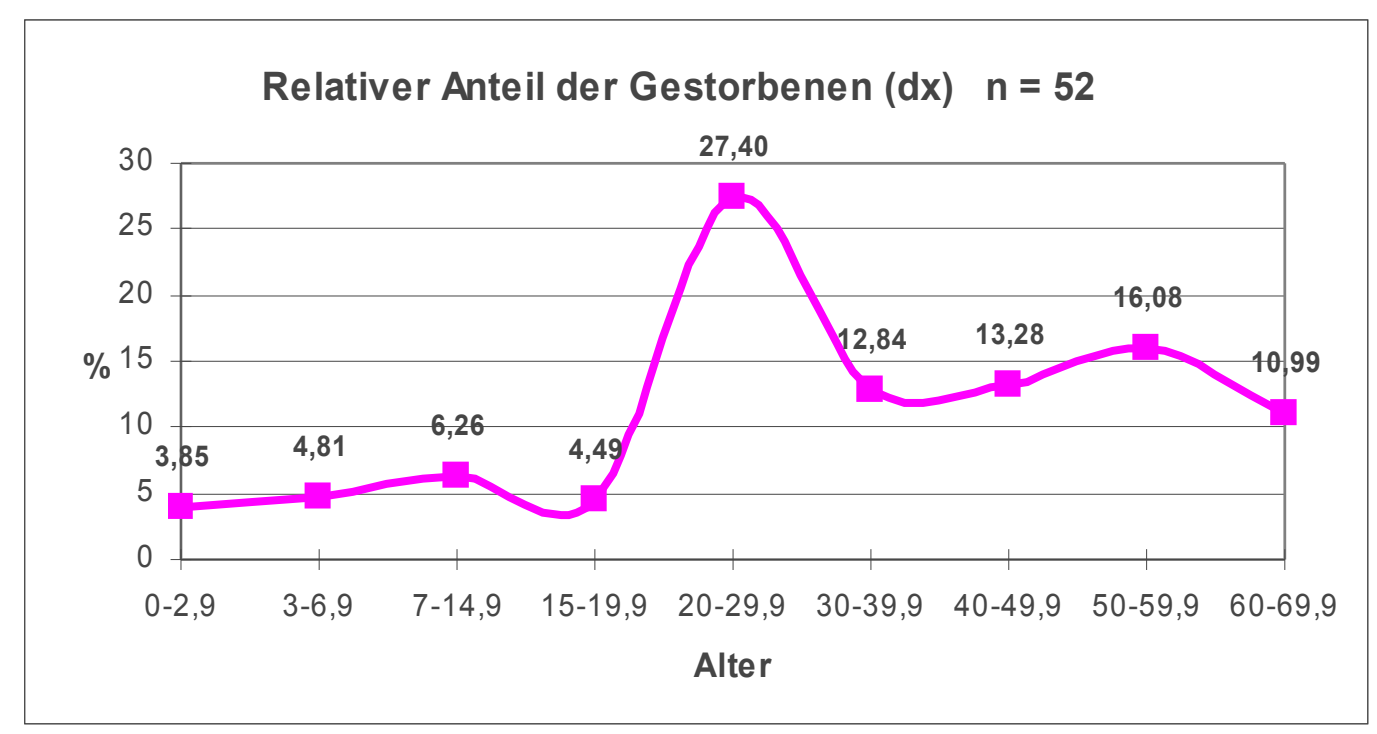

Abbildung 4: Sterbehäufigkeit der Individuen mit Schädel (7. - 9. Jhd.) 
Dabei fällt auf, dass die meisten Menschen im frühadulten Lebensalter, also von 20-29 Jahren, verstarben. Bemerkenswert ist weiterhin, dass die beobachtete hohe Säuglings- und Kindersterblichkeit anderer vergleichbarer Populationen (KÖLBL 2004) bei den vorhandenen Individuen aus Baunach nicht besteht.

Unerwartet ist ebenfalls der mit 11 Prozent relativ hohe Anteil der im senilen Altersbereich verstorbenen Personen. Die Menschen erreichten also auch vor mehr als 1000 Jahren im Frühmittelalter ein Lebensalter von über 60 Jahren, obwohl die durchschnittliche Lebenserwartung bei Geburt zu dieser Zeit nur 35,1 Jahre betrug. Allerdings stieg die Lebenserwartung nach dem Erreichen des 20 . Lebensjahres auf 41,3 Jahre an.

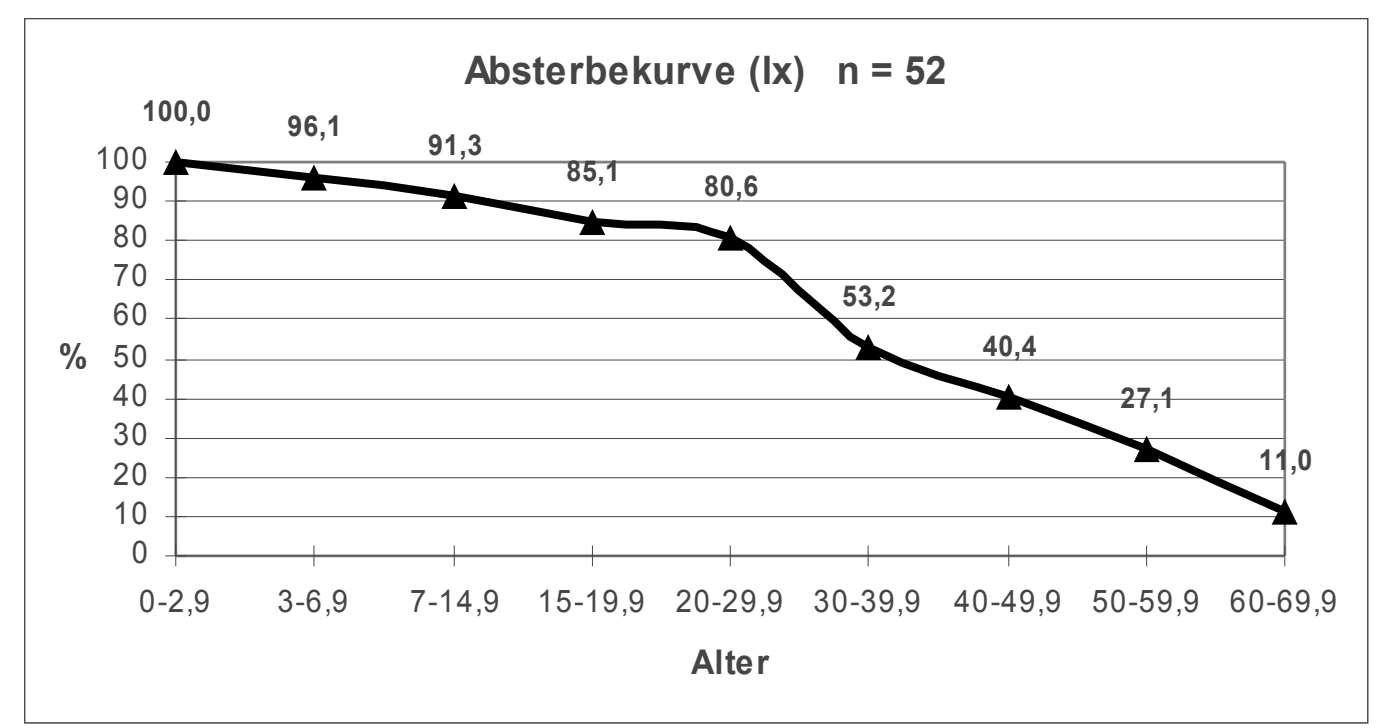

Abbildung 5: Absterbekurve der Individuen mit Schädel (7. - 9. Jhd.)

Über die Zahl der Überlebenden informiert die sogenannte Absterbekurve (Abb. 5), die für Baunach einen relativ gleichmäßig fallenden Verlauf zeigt. Anhand einer Absterbekurve mit der relativen Zahl der überlebenden Individuen in den verschiedenen Altersstufen kann eine Reflektion des Gesundheitszustandes der Personen und somit der von exogenen Faktoren determinierten Lebensbedingungen erfolgen (SCHULTZ 1989). 


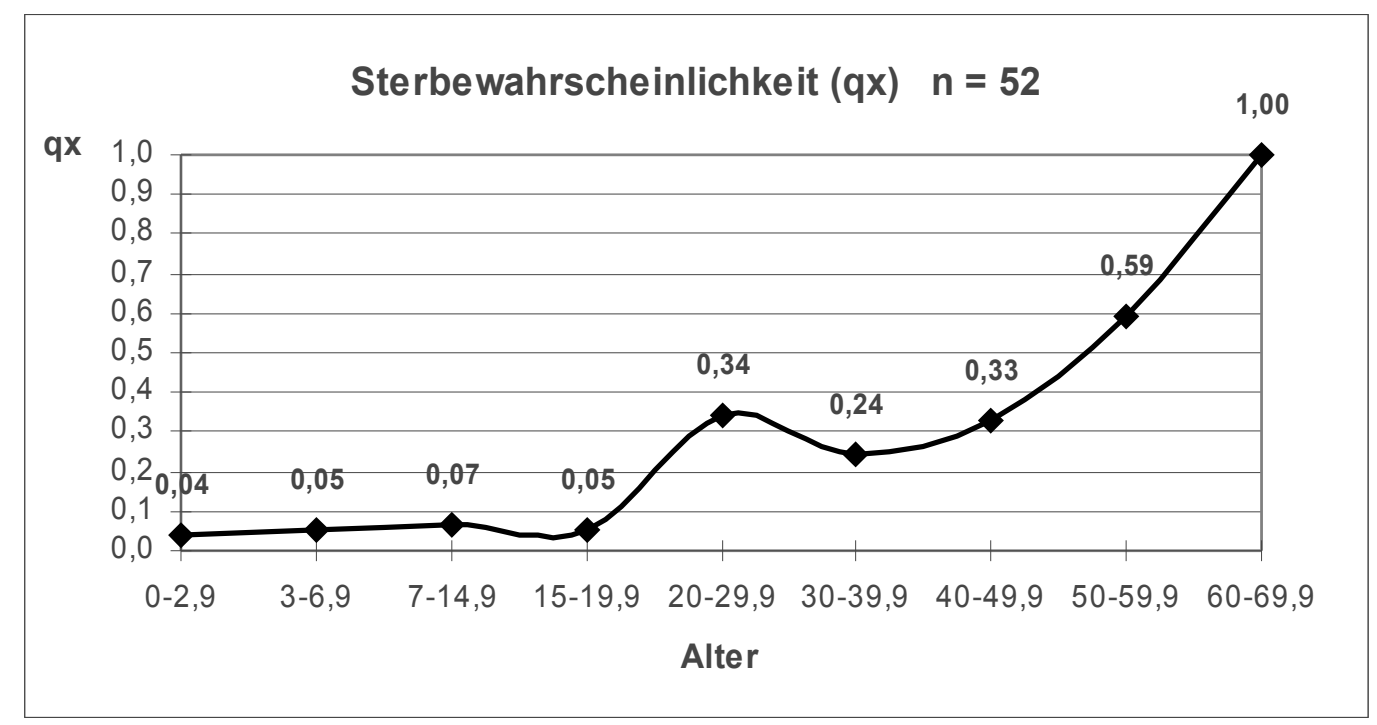

Abbildung 6: Sterbewahrscheinlichkeit der Individuen mit Schädel ( 7.-9. Jhd.)

Die größte Sterbewahrscheinlichkeit der Individuen mit Cranium aus Baunach ist in der Gruppe der frühadulten Individuen zu finden (Abb. 6). Mögliche Gründe hierfür werden bei den jeweiligen Geschlechtern im Folgenden aufgeführt.

\subsubsection{Altersverteilung der Männer}

Die durchschnittliche Lebenserwartung der Männer lag bei der Geburt bei 43,6 Jahren und somit deutlich über dem entsprechenden Wert der Baunacher Gesamtpopulation. In der statistischen Auswertung des Sterbealters der Männer zeigen sich folgende Ergebnisse: 


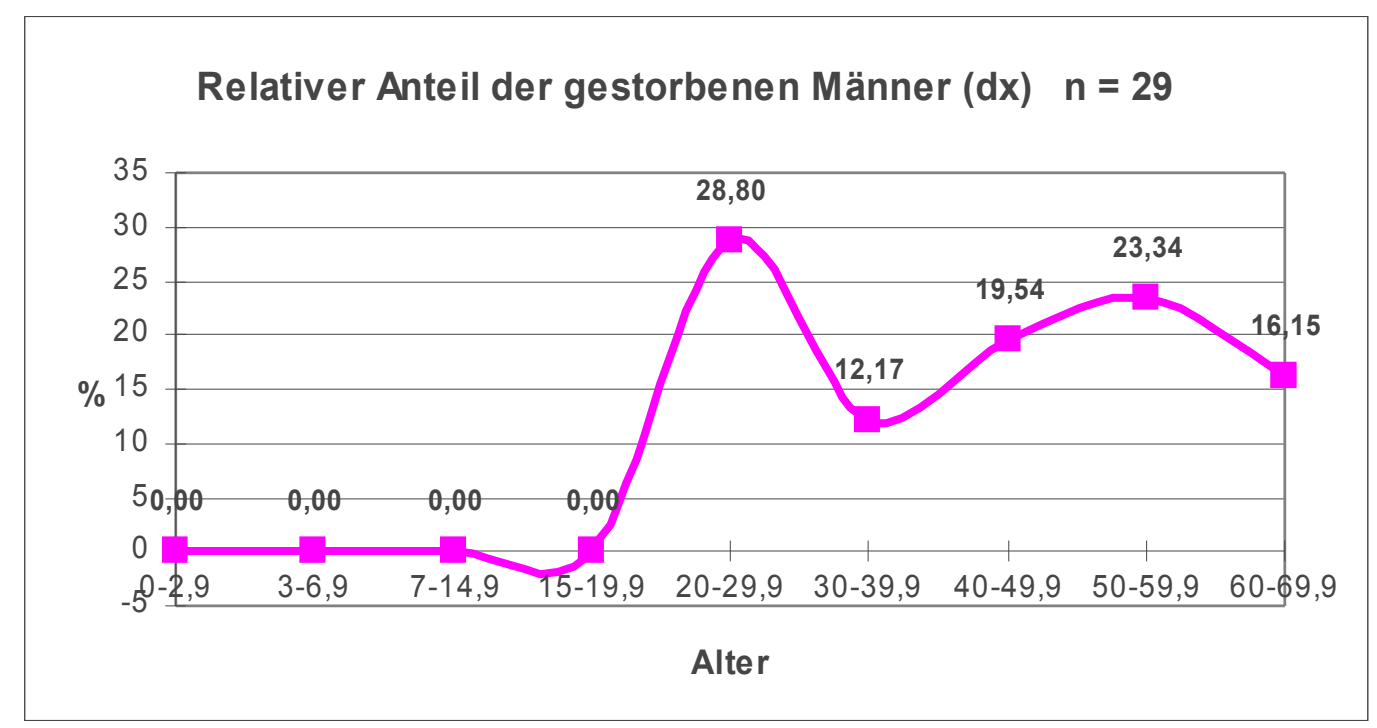

Abbildung 7: Relativer Anteil der gestorbenen Männer (7. - 9. Jhd.)

Der Anteil der frühadult verstorbenen Männer ist ähnlich wie in der Gesamtpopulation am höchsten. Die Kurve in Abbildung 7 weist einen zusätzlichen Anstieg für die im spätmaturen Lebensalter Verstorbenen auf. Die Gruppe der im senilen Altersbereich verstorbenen Männer ist größer als die Gruppe der spätadult verstorbenen Männer. War also der frühadulte Lebensabschnitt überlebt, stieg die Wahrscheinlichkeit, ein höheres Lebensalter zu erreichen, deutlich.

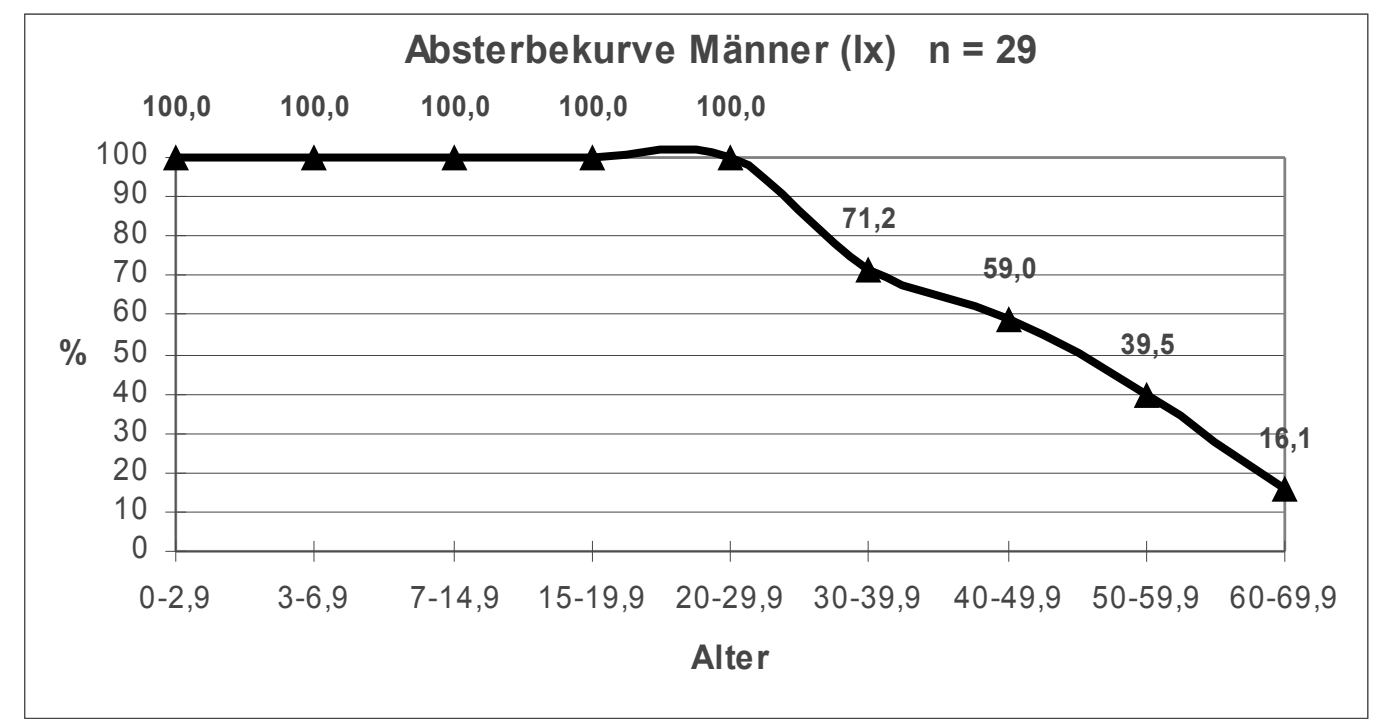

Abbildung 8: Absterbekurve der Männer (7. - 9. Jhd.) 
In der Absterbekurve (Abb. 8) wird deutlich, dass offenbar keine Männer im Kindes- und Jugendalter verstorben sind.

Diese unterschiedliche Sterbewahrscheinlichkeit der einzelnen Altersklassen demonstriert auch Abbildung 9. Der Tiefpunkt der Kurve im spätadulten Lebensabschnitt zeigt, dass in diesem Alter für Männer die niedrigste Wahrscheinlichkeit $\mathrm{zu}$ sterben bestand.

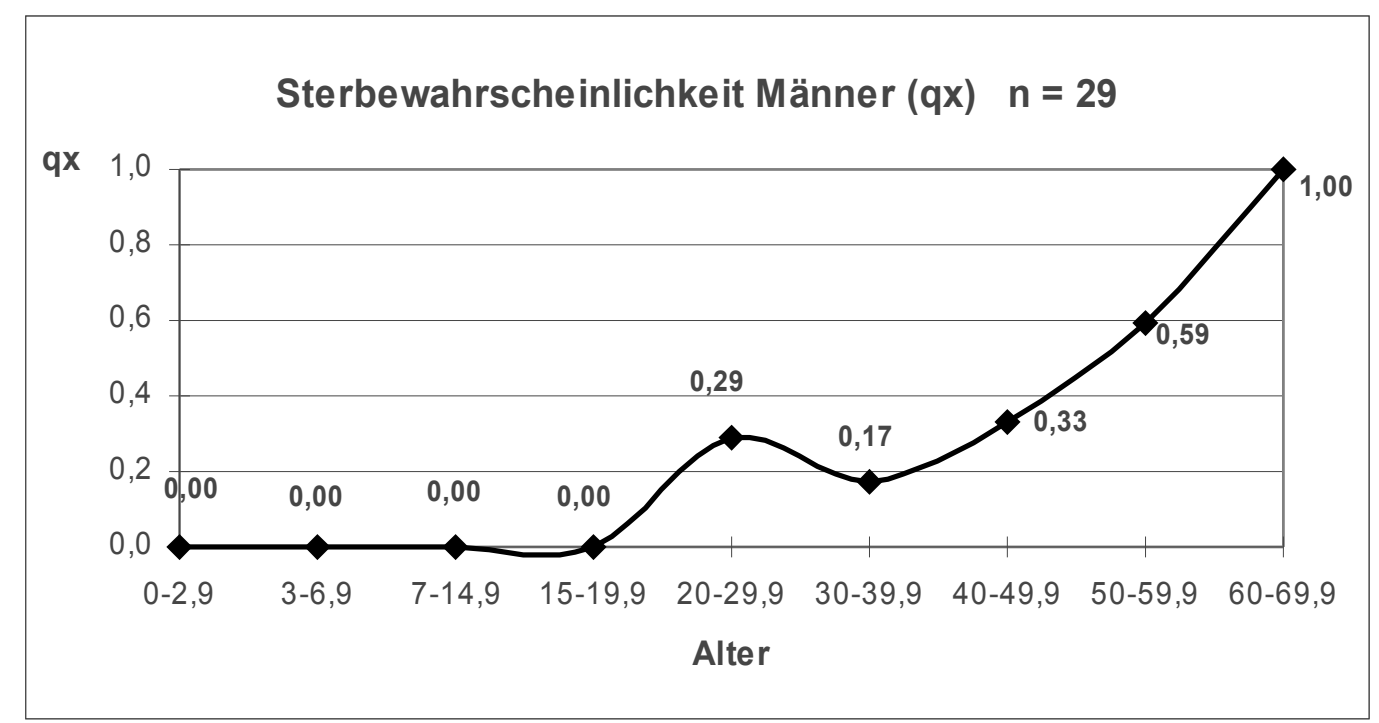

Abbildung 9: Sterbewahrscheinlichkeit der Männer (7. - 9. Jhd.)

\subsubsection{Altersverteilung der Frauen}

Eine Frau hatte bei der Geburt eine durchschnittliche Lebenserwartung von 33,0 Jahren. Allerdings erhöhte sich diese ab dem erreichten 15. Lebensjahr auf 34,1 Jahre und ab dem erreichten 20. Lebensjahr auf 37,3 Jahre. 


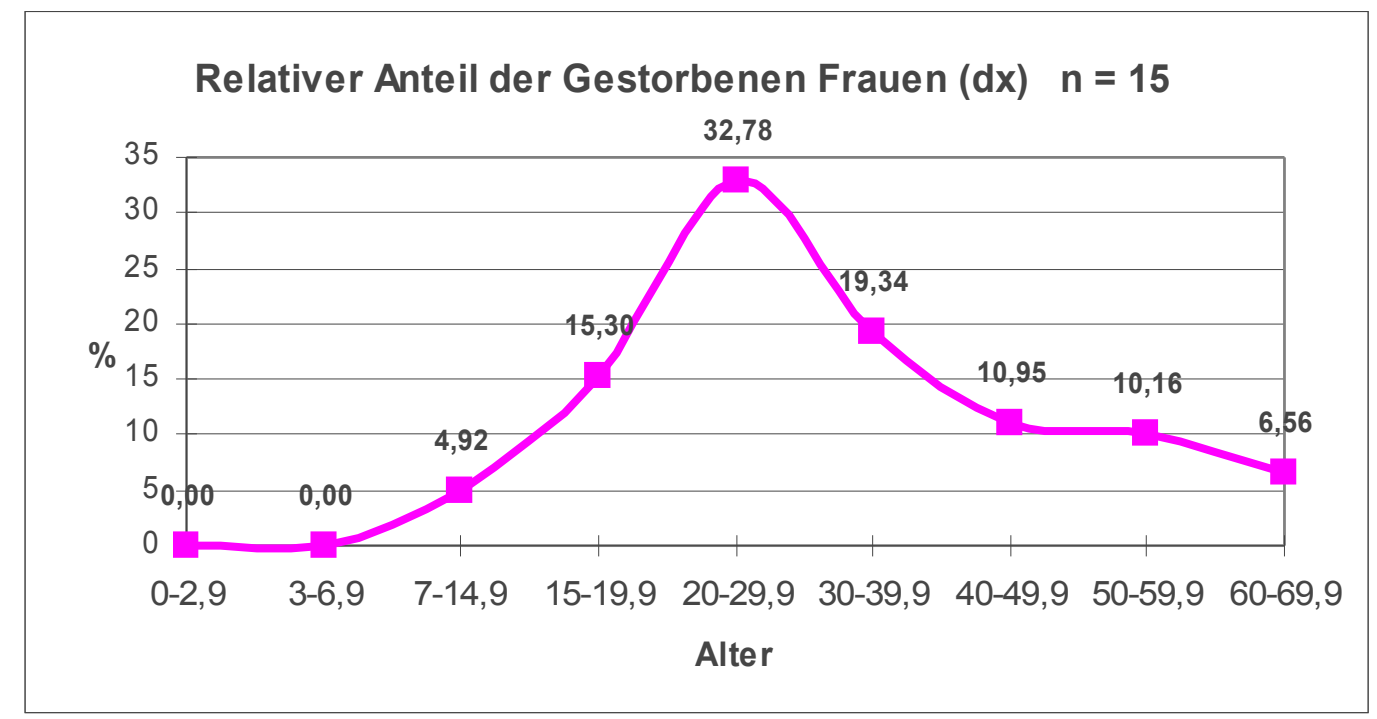

Abbildung 10: Relativer Anteil der gestorbenen Frauen (7. - 9. Jhd.)

Das Maximum der weiblichen Skelete (33 Prozent) kann dem frühadulten Lebensabschnitt zugeordnet werden. Insgesamt finden sich zwei Drittel der verstorbenen weiblichen Individuen zwischen 15 und 40 Jahren (Abb. 10). Diese Altersgruppen können als der fertile Lebensabschnitt einer Frau angesehen werden.

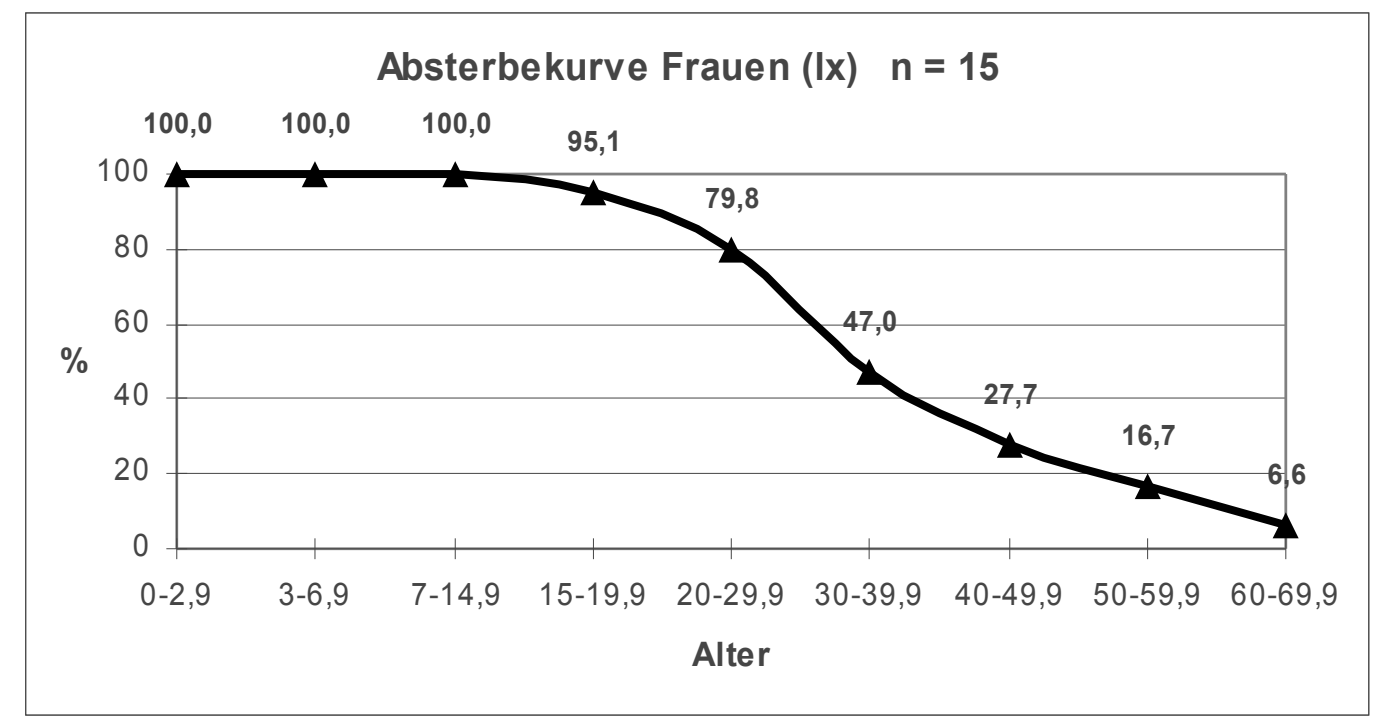

Abbildung 11: Absterbekurve der Frauen (7. - 9. Jhd.) 
Vergleichbar den männlichen Individuen erscheinen in der Absterbekurve der Frauen (Abb.11) keine Verstorbenen in den Jahren von Null bis 15. Während bei Männern im Alter von 15 bis 40 Jahren ein Abfall von knapp einem Drittel verzeichnet werden kann, steigt dieser Verlust bei Frauen um mehr als die Hälfte (53 Prozent).

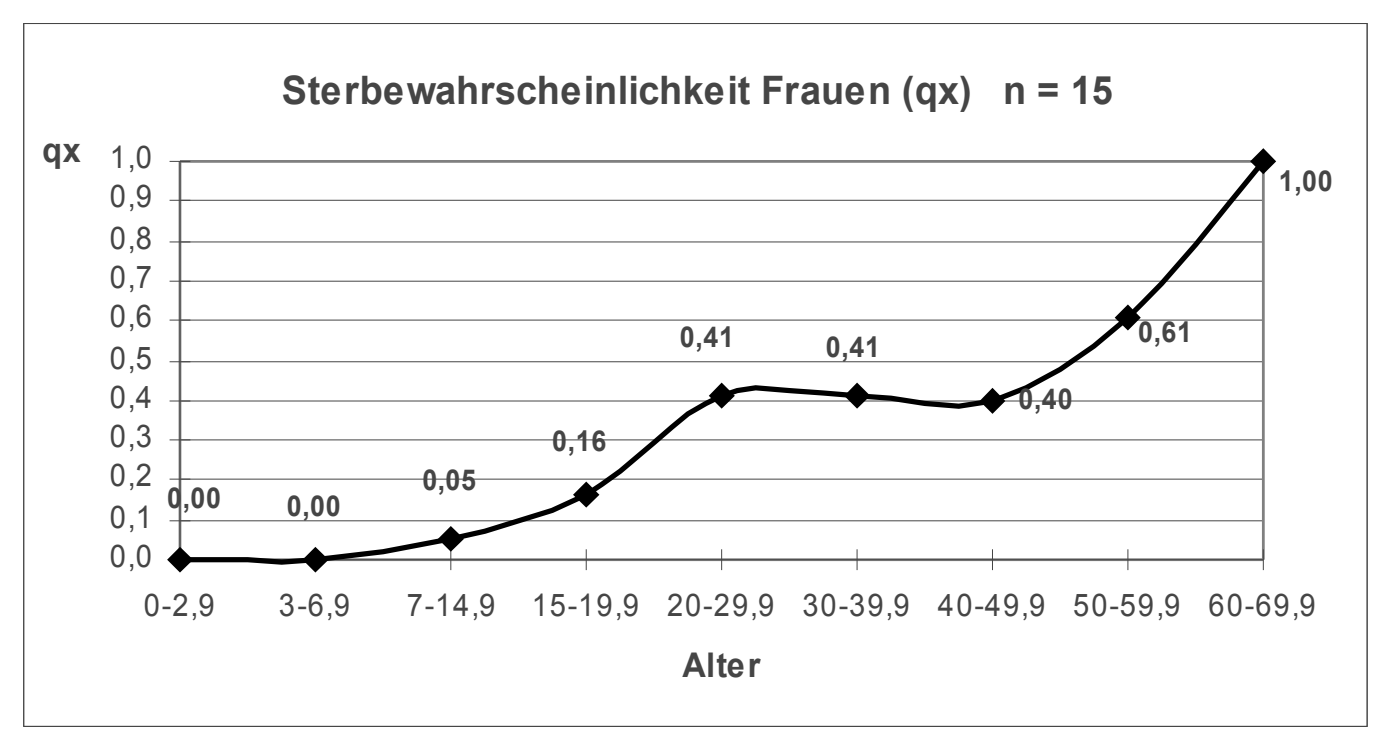

Abbildung 12: Sterbewahrscheinlichkeit der Frauen (7. - 9. Jhd.)

Die Darstellung der Sterbewahrscheinlichkeit verdeutlicht die Sterbewahrscheinlichkeit der Frauen in Bezug auf die verschiedenen Lebensaltersabschnitte (Abb.12). Im Vergleich mit der Sterbewahrscheinlichkeit der Männer besitzen Frauen mit 41 Prozent für einen Tod im frühadulten Lebensalter eine stark erhöhte Wahrscheinlichkeit gegenüber den Männern mit 29 Prozent. Der erhöhte Prozentsatz zu sterben setzt sich auch im spätadulten Alter fort. Dieser beträgt bei den Männern nur 19 Prozent, während Frauen wiederum mit 41 Prozent mehr als den doppelten Wert zeigen. Auch hier kann eine Schwangerschaft im spätadulten Lebensabschnitt als Ursache angesehen werden. 


\subsubsection{Altersverteilung nicht bestimmbarer Individuen}

Die durchschnittliche Lebenserwartung bei der Geburt der Individuen ohne Geschlechtszuordnung lag bei 8,7 Jahren. Allerdings stieg diese sprunghaft bei Erreichen des juvenilen Lebensalters. Mit 15 Jahren lag diese schon bei 26,7 Jahren. In der Abbildung 13 zeigt sich der prozentuale Anteil der Individuen nach Altersklassen. Bis auf ein spätadultes Individuum sind alle Vertreter dieser Kategorie dem Kindes- und Kleinkindalter zu zuordnen.

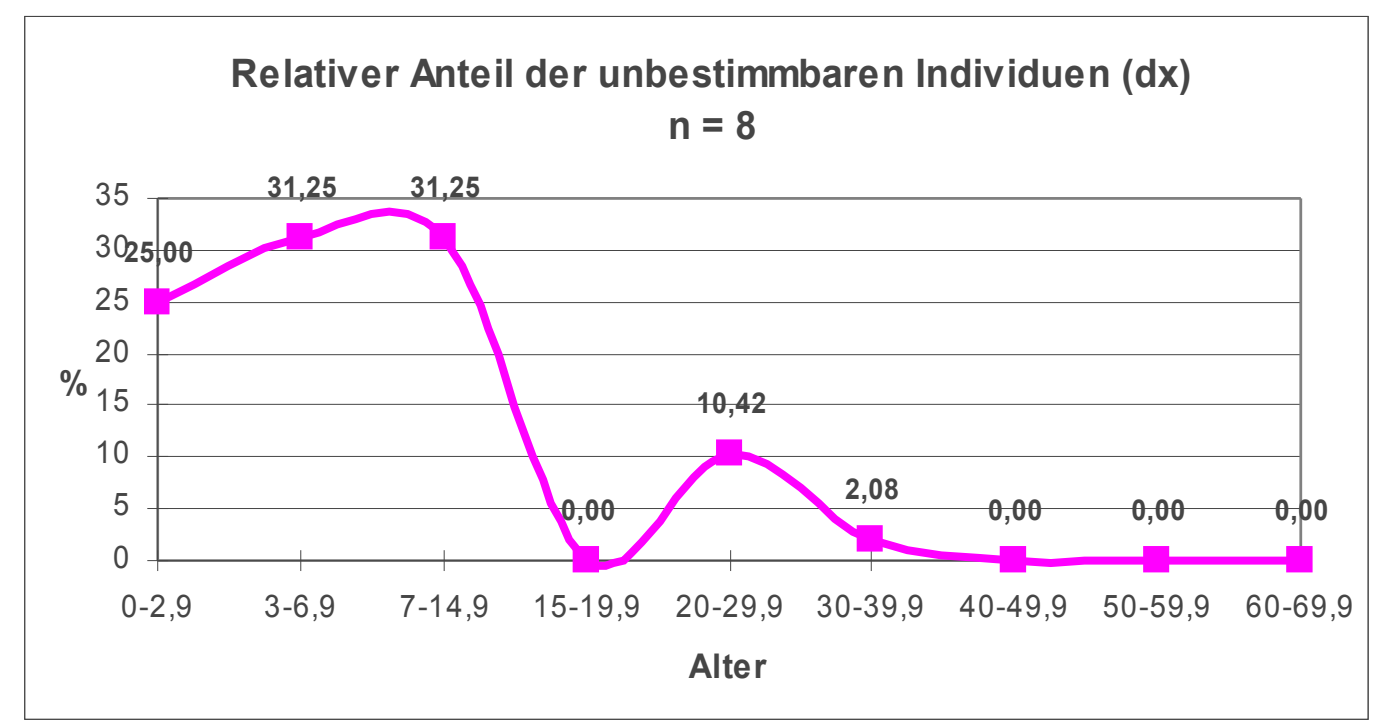

Abbildung 13: Relativer Anteil der gestorbenen unbestimmbaren Individuen (7. - 9. Jhd.)

Eine Aussage zum Verlauf der Absterbekurve ist bei nur 8 Individuen als Ausgangspunkt nur spekulativ. Man findet jedoch den größten Anteil der nicht bestimmbaren Individuen in den Gruppen Infans Ia bis Infans II (Abb. 13). Es ist $\mathrm{zu}$ sehen, dass viele Kinder verstarben (Abb. 14) und somit bis zum Erreichen eines juvenilen Alters eine große Sterbewahrscheinlichkeit bestand (Abb. 15). Ab einem juvenilen Lebensalter kann aber von Werten ausgegangen werden, welche in den Kapiteln 3.2.1 sowie 3.2.2 beschrieben worden sind. 


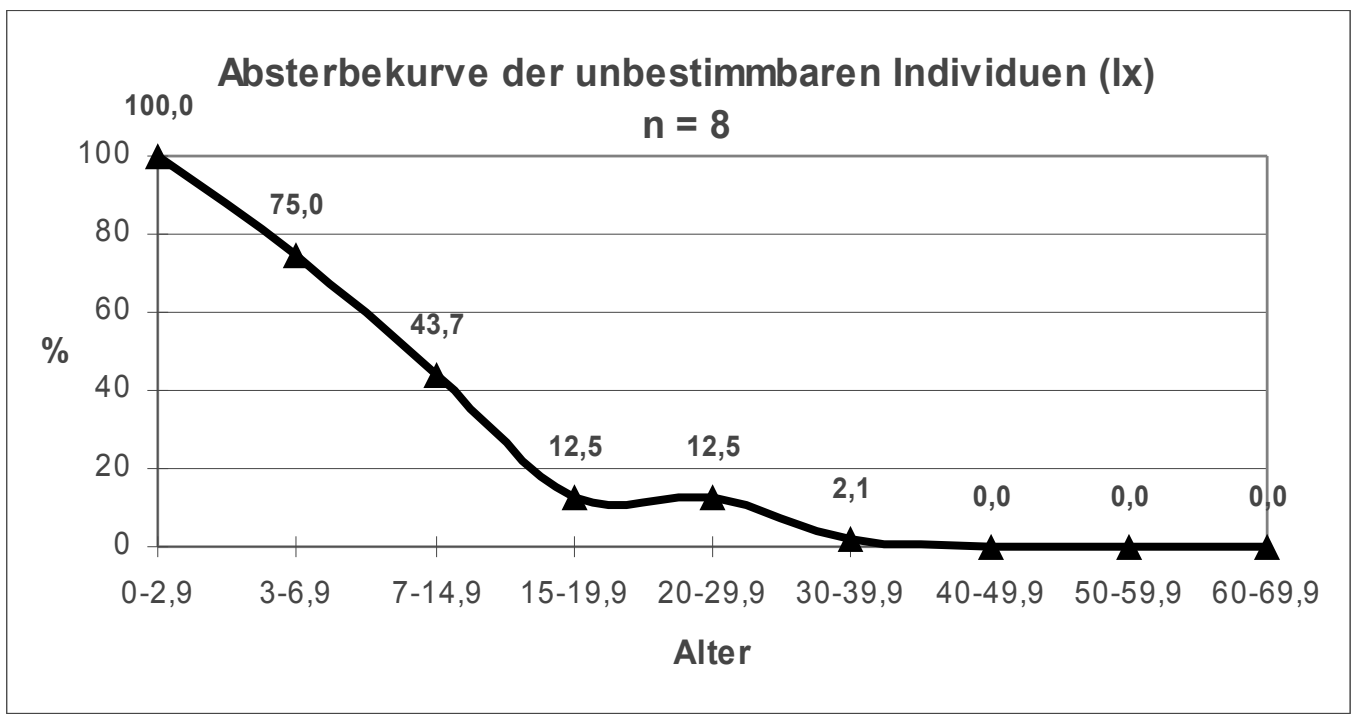

Abbildung 14: Absterbekurve der unbestimmbaren Individuen (7. - 9. Jhd.)

\section{Sterbewahrscheinlichkeit unbestimmbare Individuen (qx)$$
n=8
$$

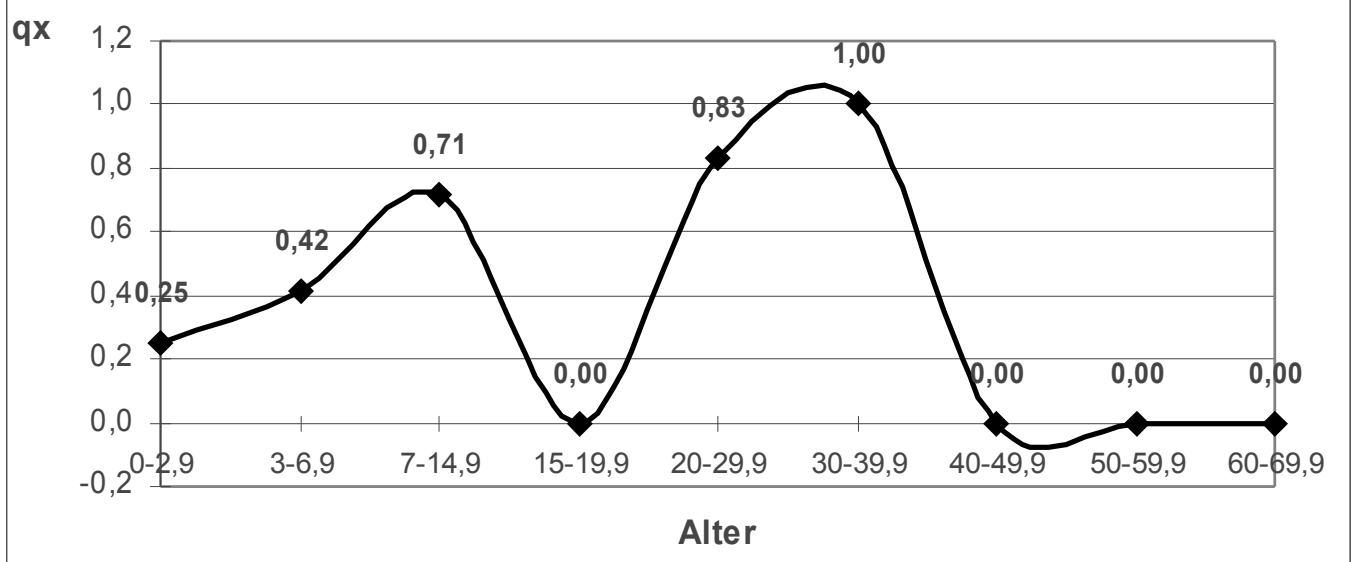

Abbildung 15: Sterbewahrscheinlichkeit der unbestimmbaren Individuen (7. - 9. Jhd.) 


\subsection{Pathologische Befunde in der Nasenhöhle}

\subsubsection{Anatomische und physiologische Grundlagen}

Die Nasenhöhle stellt den obersten Abschnitt des Respirationstraktes dar. Dieser ist - beginnend kurz hinter den kräftigen Terminalhaaren am Naseneingang zur Insektenabwehr und Filterung von gröberen Partikeln - ausgekleidet von nichtverhornendem Plattenepithel. Die knöcherne Begrenzung der Nasenhöhlen erfolgt aus Os frontale, Os nasale, Vomer, Maxilla, Os ethmoidale, Os sphenoidale, der Concha nasalis inferior und den Cartilagines nasi als Restteil des Septums und der knorpeligen Nasenspitze.

Die physiologische Aufgabe der Nasenhöhle ist die Vorbereitung der Atemluft für die unteren Atemwege (vergleiche Lehrbücher Innere Medizin, Anatomie). Dazu gehören das Anwärmen, das Anfeuchten, die Reinigung von Staubteilchen und Bakterien und letztendlich durch olfaktorische Nervenzellen auch eine Prüfung auf die chemische Beschaffenheit der Atemluft.

Das Erwärmen der Luft lässt sich am Beispiel eines „Warmwasserheizkörpers“ verdeutlichen. Die Nasenmuscheln stellen die Heizrippen dar, die durch das Blut erwärmt werden. Auch beim Befeuchten der Luft spielen die Nasenmuscheln eine wichtige Rolle. Ihre gefäßreiche Schleimhaut produziert Wasserdampf und reichert so die Atemluft mit Feuchtigkeit an. Zur groben Filterung der Atemluft findet man Terminalhaare im Bereich der durch Nasenknorpel begrenzten Nase. Gebunden werden feinere Partikel in einem dünnen Schleimbelag, der eine zusätzliche Barriere zur Infektabwehr darstellt. Im Rachen findet sich schließlich ein Ring aus lymphatischem Gewebe, der sowohl den respiratorischen als auch den gastrointestinalen Trakt abschirmt. Diese auch als Waldeyer'scher Rachenring bezeichnete Formation besteht aus der Tonsilla pharyngealis, der Tonsilla tubaria, der Tonsilla lingualis und der Tonsilla palatina. Diese sind jeweils paarig angelegt. Die chemische Zusammensetzung wird durch Filamente des Nervus olfactorius, dem Riechnerv, überprüft. Dieses geschieht, um beispielsweise auf eventuell giftige Gase oder auch auf ungenießbare Speisen aufmerksam zu machen. 
Im Bereich der Nasenhöhle befindet sich der Übergang zwischen innerer und äußerer Körperoberfläche.

\subsubsection{Erkrankungen in der Nasenhöhle}

Die Entzündung der Nasenschleimhaut, die Rhinitis, gehört zu den häufigsten infektiösen Affektionen und trägt meist den Charakter eines banalen Schnupfens. Sämtliche Lebensalter sind davon betroffen. Eine deutliche Abhängigkeit von der Jahreszeit ist nachweisbar (JÜRGENSEN 1889).

Meist ist die Infektion nur auf die Oberfläche, das respiratorische Flimmerepithel, beschränkt. Bei fortgeschrittener oder chronischer Erkrankung kann sich die Infektion - beispielsweise eine granulomatöse Rhinitis - jedoch auch auf die tiefer liegenden Schichten des Knorpel- und Knochensystems ausweiten (ZIEGLER 1898). Diese sind einer paläopathologischen Auswertung zugänglich.

In der Mehrzahl der Fälle wird eine Rhinitis durch eine Virusinfektion ausgelöst und verläuft ohne weitere Komplikationen. Die Symptome einer unkomplizierten Rhinitis sind wässrige Sekretion und eine Behinderung der Nasenatmung durch Anschwellen der Schleimhaut. Ein banaler Virusinfekt der Nasenschleimhaut stellt für ein immunkompetentes Individuum allerdings meist kein Problem dar. Trotzdem kann sich diese Infektion bei gewissen Viren (Viren sind organotrop, d.h. sie siedeln nur in einem bestimmten Gewebetyp, zum Beispiel der respiratorischen Schleimhaut) auch auf die unteren Atemwege ausdehnen und zu einer Bronchitis oder schlimmer zu einer viralen Lungenentzündung führen. Allerdings geschieht dies fast ausschließlich bei Kleinkindern und älteren Menschen, also bei Vorliegen eines insuffizienten Immunsystems.

Wenn aber, bedingt durch den Virusinfekt und die damit verbundene Irritation der Nasenschleimhaut, auf einer abwehrgeschwächten Schleimhaut zusätzlich Bakterien siedeln, kann dies zu weiter reichenden Konsequenzen führen. Man bezeichnet diesen Prozess auch als Superinfektion. 
Die Folgen einer bakteriellen Besiedlung kann man grob in drei Klassen einteilen:

1. Infektion der Atemwege

2. Hyperplasie der Schleimhäute mit eitriger Sekretion

3. Ausbreiten der Infektion über die Atemwege hinaus.

Bei einer Infektion der oberen Atemwege besteht immer die Gefahr einer absteigenden Entzündung. So kann eine schwere Lungenentzündung oder auch eine Entzündung der Stirn-, Kiefer- oder Keilbeinhöhle sowie der Siebbeinzellen aus einer bakteriell superinfizierten Rhinitis erwachsen. Bei chronischer Nasen- bzw. Nasennebenhöhlenentzündung kann diese ständig Keime in die Atemwege streuen und auch dort eine Entzündung unterhalten. Man spricht dann von einem sinubronchialem Syndrom (Abb. 16). Natürlich können auch bronchiale Infektionen aufsteigen und eine Sinusitis unterhalten.

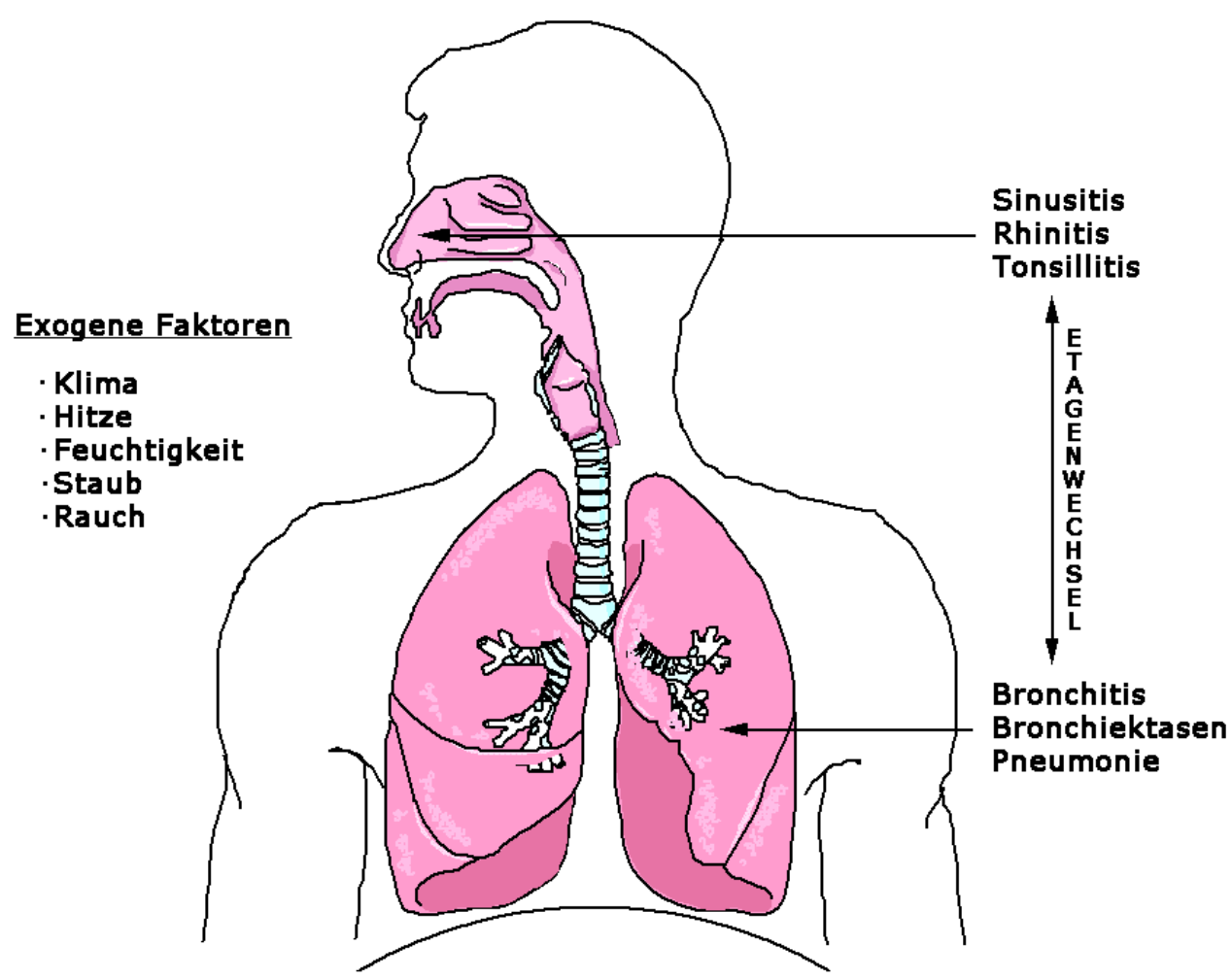

Abbildung 16: Begünstigende Faktoren und Wechselwirkungen eines sinu bronchialen Syndroms

(Quelle:Modifiziert von http://www.ambulancetechnicianstudy.co.uk/images/RESPIR.gif) 
Bei bakteriellen Infektionen der Nasenhöhle besteht auch immer die Gefahr einer Ausbreitung in benachbarte Schädelstrukturen mit gelegentlich dramatischen Folgen. So kann eine Infektion sich über die Nasenhöhle auf die Kieferhöhle ausbreiten. Dort verursacht sie dann durch die enge räumliche Nähe zur Orbita eine Orbitalphlegmone oder eine Entzündung des Tränennasengangs.

Eine durch bakterielle Rhinitis verursachte Infektion der Stirnhöhle kann durch Fortleitung der Entzündung (per continuitatem, hämatogen, Fisteln) mit Beteiligung des Gehirns ohne moderne Medikamente und chirurgische Interventionen auch den Tod des Individuums durch Sinusthrombose, Meningitis oder Enzephalitis zur Folge haben (YOUNIS 2002). Somit kann ein zunächst simpler Schnupfen durch Ausbreitung der Infektion eine ernste vitale Bedrohung eines Individuums darstellen.

Die paläopathologische Untersuchung der Nasenhöhle und der sie umgebenden Strukturen kann also wertvolle Hinweise für Epidemiologie und die Ausprägungsgrade dieser Krankheitsgruppe liefern .

\subsubsection{Ergebnisse Nasenhöhle}

Es wurden alle Individuen mit mindestens einer befundbaren Nasenhöhle untersucht. Ein Individuum wurde als krank bewertet, bei dem sich Spuren eines pathologischen Prozesses zeigten. In Abbildung 17 erkennt man eine starke Dominanz an männlichen Individuen. 


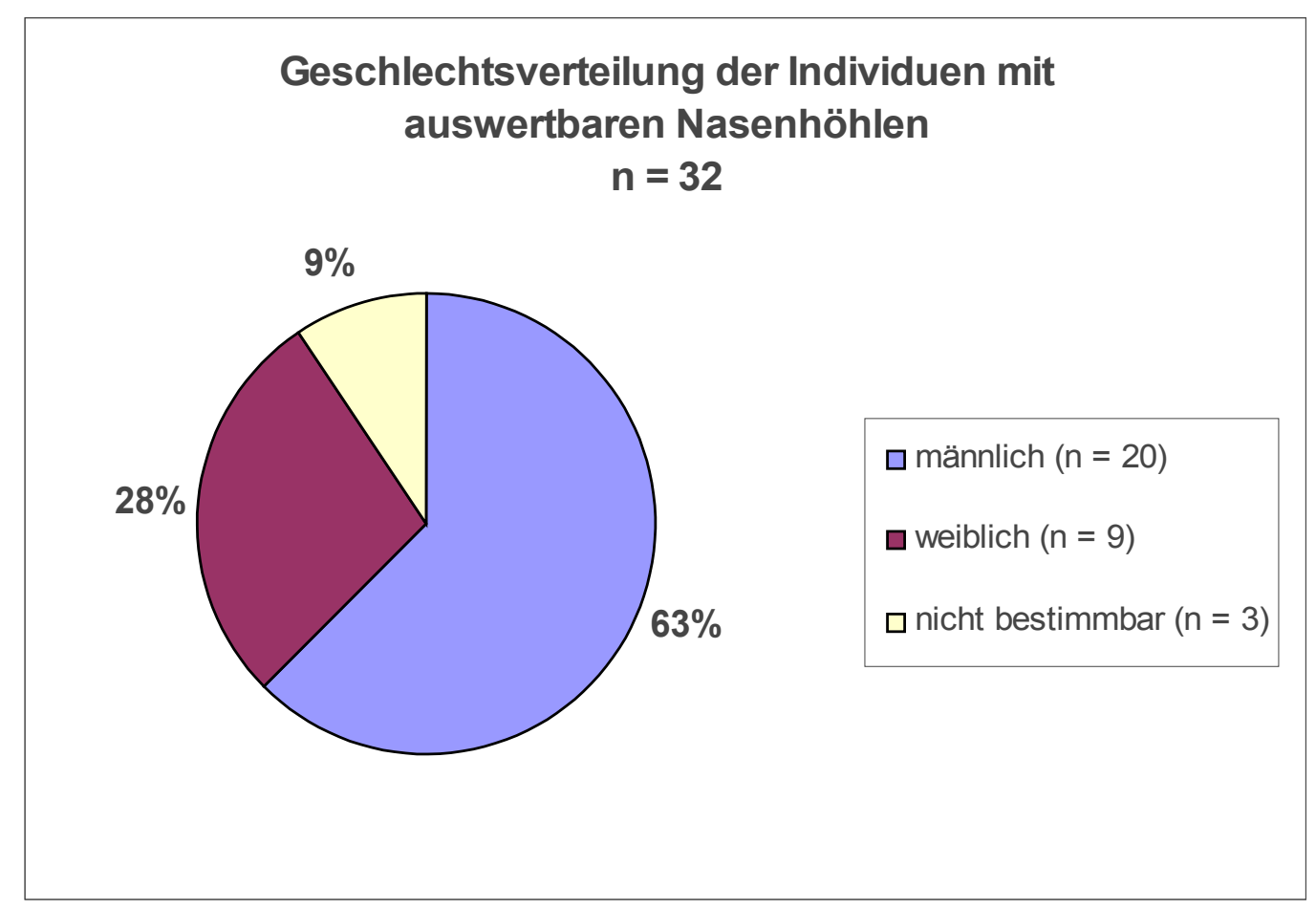

Abbildung 17: Geschlechtsverteilung der Nasenhöhlen

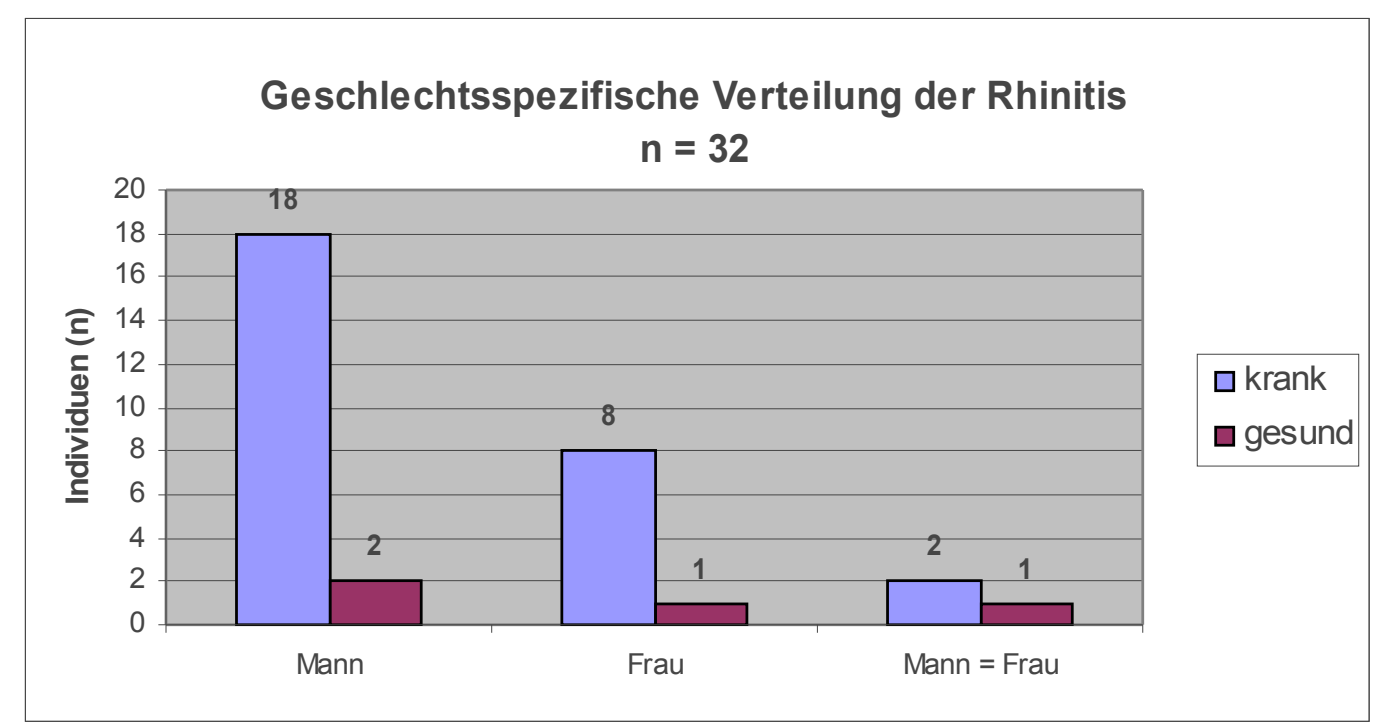

Abbildung 18: Geschlechtsspezifische Verteilung der Nasenhöhlenentzündungen 
Als Spuren eines pathologischen Prozesses gelten eine über die Norm poröse Knochenoberfläche, deutlich vermehrte Gefäßimpressionen oder poröse Platten oder Neubildungen als Spuren einer durch Nasenschleimhautreizung hervorgerufenen pathologischen Antwort der Knochenhaut. Die für die einzelnen Geschlechter gefundenen Fallzahlen sind in Abbildung 18 aufgeführt.

Aus diesen Zahlen ergeben sich auf die Geschlechter bezogen folgende Erkrankungshäufigkeiten (Abbildung 19):

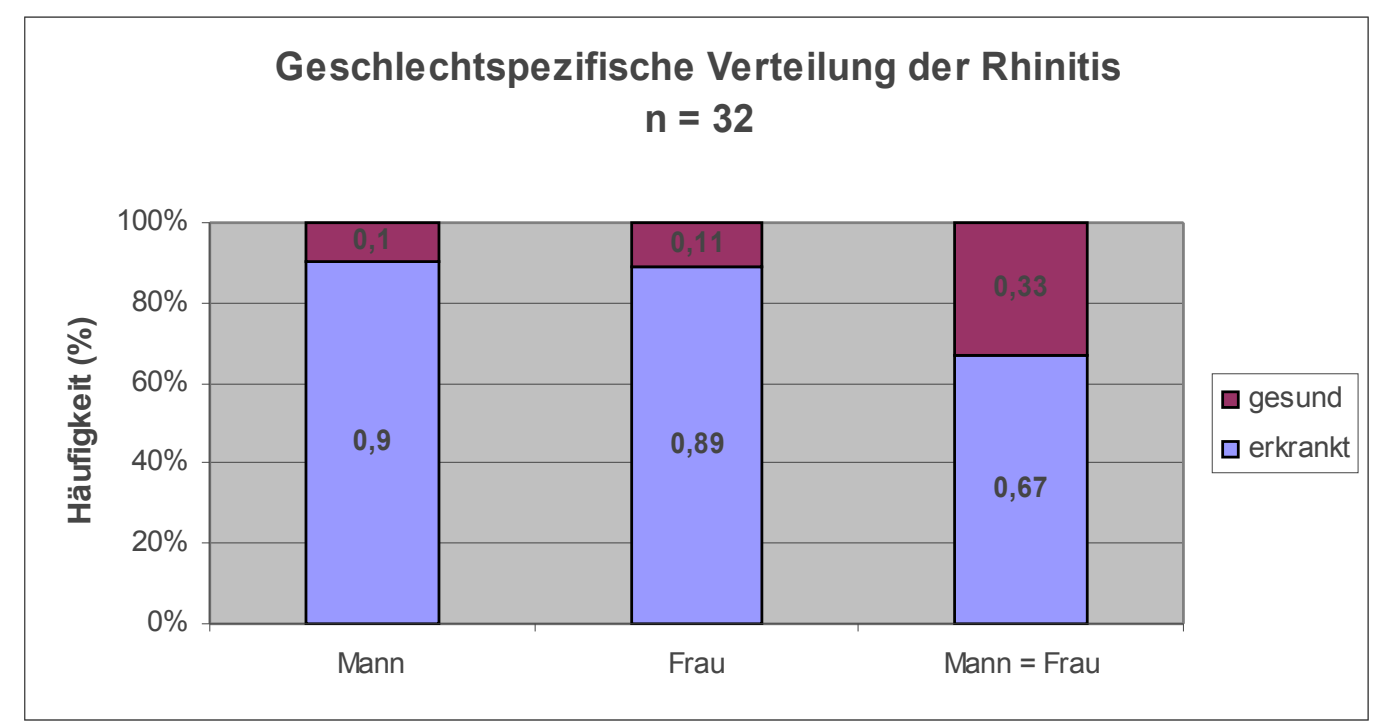

Abbildung 19: Geschlechtsspezifische Erkrankungshäufigkeit der Nasenhöhlenentzündung

Auffällig ist eine ähnliche Krankheitshäufigkeit um 90 Prozent bei Männern und Frauen. Die Gruppe der nicht bestimmbaren Individuen zeigt mit 67 Prozent einen etwas niedrigeren Anteil der Erkrankungen. 


\subsection{Pathologische Befunde in den Nasennebenhöhlen}

\subsubsection{Anatomische und physiologische Grundlagen}

Als Nasennebenhöhlen werden die luftgefüllten Räume in den Nachbarknochen der Nasenhöhle bezeichnet. Diese pneumatischen Räume sind wie die Nasenhöhle von Nasenschleimhaut bedeckt. Histologisch zeigt sich ein zweireihiges Flimmerepithel. Im Gegensatz zur Schleimhaut der Cavitates nasi finden sich in den Sinus paranasales kein ausgeprägtes submuköses Bindegewegebe und keine physiologisch angelegten Venenplexus und arteriovenöse Anastomosen (LEONHARDT 1990). In der Regel sind also die Wände der Nasennebenhöhlen physiologisch glatt.

Nasennebenhöhlen sind ursprünglich bei der Geburt aus Gründen der Raumersparnis und Geburtserleichterung im Mutterleib und Geburtserleichterung nicht vorhanden und entwickeln sich erst in den Jahren der Kindheit und des Wachstums. Sie entstehen durch Ausstülpungen der Nasenschleimhaut und Resorption der entsprechenden Knochen während der ersten Lebensjahre und schaffen somit einen neuen, luftgefüllten Raum (KLOSE, GRUNDMANN 1953).

Dieser pneumatisierte Knochen dient mehreren Aufgaben. Unter anderem wird dadurch das Schädelgewicht bei größerem Volumen durch diese Art Leichtbau deutlich reduziert. Durch das höhere Volumen wird gleichzeitig auch eine Oberflächenvergrößerung der Nasenschleimhaut erreicht, so dass diese in größerem Umfang ihren Aufgaben nachkommen kann. So wird also eine stärkere Erwärmung und Anfeuchtung der Atemluft sowie eine über Schleimhäute vermittelte Immunbarriere für über die Atemluft eindringende Keime erreicht. Auch als vergrößerter Resonanzraum unserer Sprache spielen die Nasennebenhöhlen eine wichtige Rolle.

Die Nasennebenhöhlen sind jeweils über einen dünnen Kanal mit der Nasenhöhle verbunden. Über diesen Kanal kommunizieren die luftgefüllten Sinus mit dem Cavum nasi. Ein Eindringen von Infektionen, ausgehend vom Ostium naturale, über diese Verbindung ist bei Entzündungen der Atemwege möglich. 
In Abbildung 20 sind die topographischen Verhältnisse der Lagebeziehungen der Nasennebenhöhlen dargestellt.

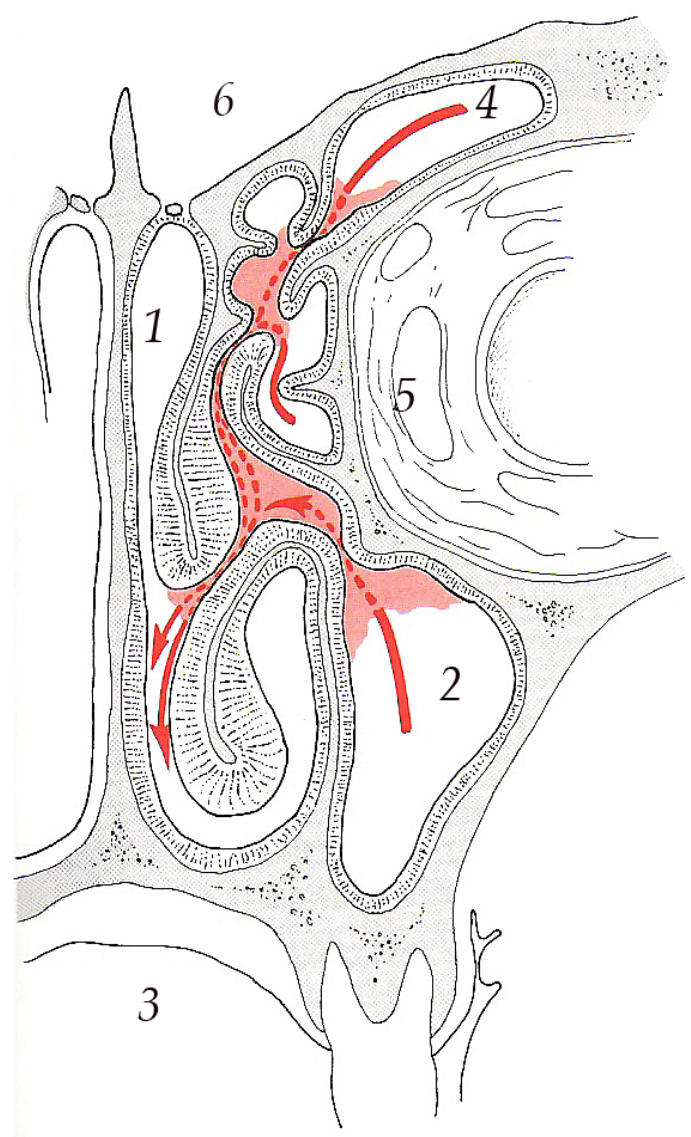

Abbildung 20: Übersicht topographische Beziehung der Nasenhöhle (Quelle: Kastenbauer, Tardy jr. 1995; S.455)

1. Nasenhöhle

2. Kieferhöhle

3. Mundhöhle

4. Stirnhöhle

5. Orbita

6. Endocranium 
Die Ausprägung der Nasennebenhöhlen erfolgt nicht genau seitengleich. Es kann also durch Pneumatisationshemmung ein Sinus schwächer als der kontralaterale ausgeprägt sein. Bei angeborenen oder durch Krankheit bedingten Minderausprägungen oder fehlenden Anlagen der Nasennebenhöhlen werden ihre physiologischen Funktionen wie oben beschrieben nicht oder nur vermindert ausgeführt. Somit resultiert eine erhöhte Anfälligkeit für respiratorische Infekte.

\subsubsection{Erkrankungen der Nasennebenhöhlen}

Infektionen der Nasennebenhöhlen (Sinusitis) können auf vielfältigen Wegen erfolgen. Die Hauptursache stellen über die Atemluft aufgenommene Keime wie Pneumokokken, Haemophilus influenzae, Staphylokokken oder Streptokokken dar (SIMON, STILLE 2000). Auch über Tröpfcheninfektion aufgenommenes virales Infektionsmaterial kann eine Entzündung der Schleimhaut verursachen. Diese aerogen bedingten Infektionen werden durch nasskaltes Klima deutlich begünstigt.

Die Population aus Baunach kann diesem Klima eindeutig zugeordnet werden. Auch räumlich enges Zusammenleben vieler Menschen erhöht die Rate von aerogenen Infektionen. Bei den Baunacher Individuen ist davon auszugehen, dass sie wie in der damaligen Zeit üblich als Großfamilie in einem Haus lebten (BECK, STEUER 1997). Es ist anzunehmen, dass eine vielköpfige Familie unter einem Dach zusammen wohnte und respiratorische Infektionen unter den Familienmitgliedern untereinander relativ schnell weitergegeben wurden.

Über den Blutkreislauf können septische Herde in den Sinus paranasales entstehen. Möglich ist auch eine Ausbreitung der Infektion auf direktem Wege durch den Knochen, also „per continuitatem“, beispielsweise ausgehend von vereiterten Zahnwurzeln in den Sinus maxillaris. Dabei entstehen durch die Eiteransammlung zur Druckentlastung Fistelgänge, welche infektiöses Material in den Sinus bringen. Bei räumlich sehr enger Beziehung der Kieferhöhle zum Zahnhalteapparat sind Erkrankungen der Zähne sowie des Zahnhalteapparates somit als begünsti- 
gende Faktoren anzusehen. Also sind alle begünstigenden Faktoren für eine Zahnwurzelentzündung wie Karies, Stomatitis oder Skorbut auch gleichzeitig Risikofaktoren für eine Sinusitis maxillaris (Abb.21).

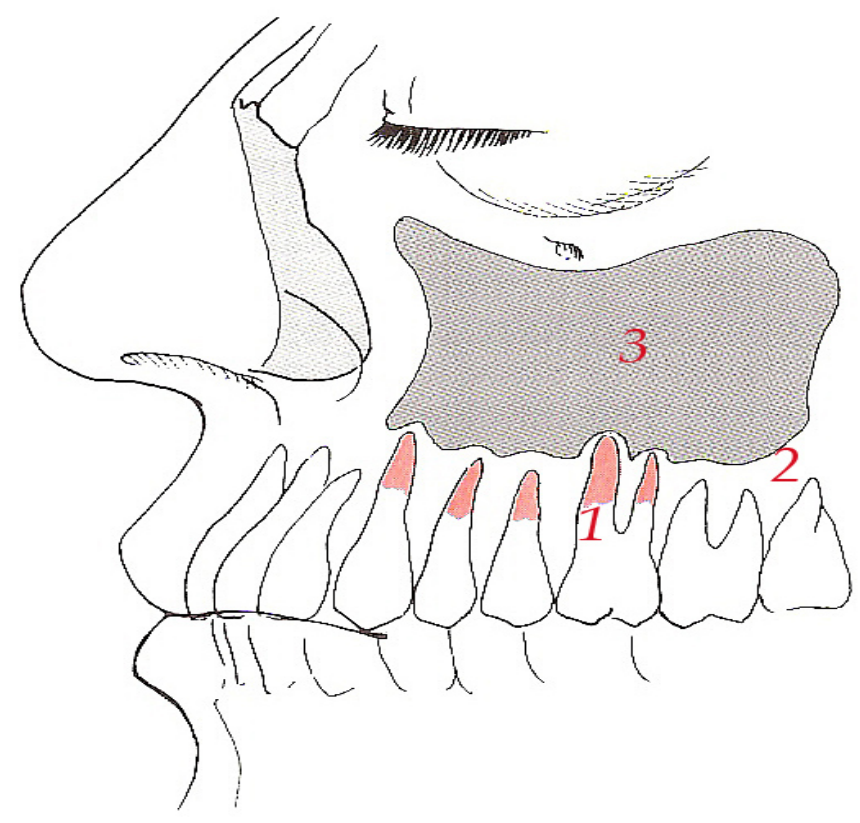

Abbildung 21: Beziehungen des Zahnhalteapparates des Oberkiefers zur Kieferhöhle beim Erwachsenen (Quelle: Kastenbauer, Tardy jr. 1995; S.455)

1. Zahnhalteapparat

2. Oberkiefer

3. Sinus maxillaris

Bei einer entzündeten Nasennebenhöhle ist die Infektion sehr gut räumlich abgeschottet und die Infektionserreger können sich demzufolge auch sehr gut entwickeln. Durch eine Ausbreitung der Infektion können von einer infizierten Nasennebenhöhle aus auch alle anderen Sinus infiziert werden. Die Folge ist eine Pansinusitis, die mit starkem Fieber und starken Schmerzen einhergeht. Da direkt hinter dem Sinus frontalis die Hirnhäute und das Gehirn liegen, wird durch eine eitrige Stirnhöhlenentzündung gelegentlich auch der Schädelinnenraum durch 
Einschmelzung mit Fistelbildung erreicht. Bedingt durch Anastomosen im venösen Abfluss ist auch eine hämatogene Ausstreuung der Erreger vom Sinus frontalis auf den endocraniellen Raum möglich. Die Folge kann eine Meningitis, eine Enzephalitis oder eine Sinusthrombose sein.

Durch die physiologisch angelegte Verbindung von Nasennebenhöhle und Mittelohr (Tuba auditiva) können Erkrankungen des Respirationstraktes auch das Mittelohr erreichen. Wieder sind Kinder stärker gefährdet, da deren Tubeneingang noch größer und der Weg bis ins Mittelohr kürzer ist als bei Erwachsenen. Hat die Infektion das Mittelohr erreicht, kann eine Otitis media, eine Mittelohrentzündung, entstehen. Hauptkomplikationen dieser Krankheit sind Meningitis, Sepsis und bleibende Hörschäden.

Auch die Orbita ist nur durch eine hauchdünne Knochenmembran von der Kieferhöhle getrennt. So kann sich eine Infektion wieder durch eitrige Einschmelzung oder hämatogen leicht auf diese ausdehnen. Die Folge wäre eine gefährliche und schmerzhafte Orbitalphlegmone und/oder eine Dakryozystitis mit begleitender Konjunktivitis. Eine deutliche Beeinträchtigung des Sehvermögens bei chronischem Verlauf ist möglich.

Ein Beispiel für eine ausgeprägte abszedierte Sinusitis maxillaris mit Fistel zur Mundhöhle wird in den Bildern neun bis zwölf im Anhang gezeigt.

Es wird also deutlich, welche weitreichenden Folgen für das alltägliche Leben eines Menschen durch eine an sich banale Infektion der Nebenhöhlen entstehen können. Beispiele für Ausbreitungsrichtungen einer Sinusitis paranasalis sind in Abbildung 22 dargestellt. 


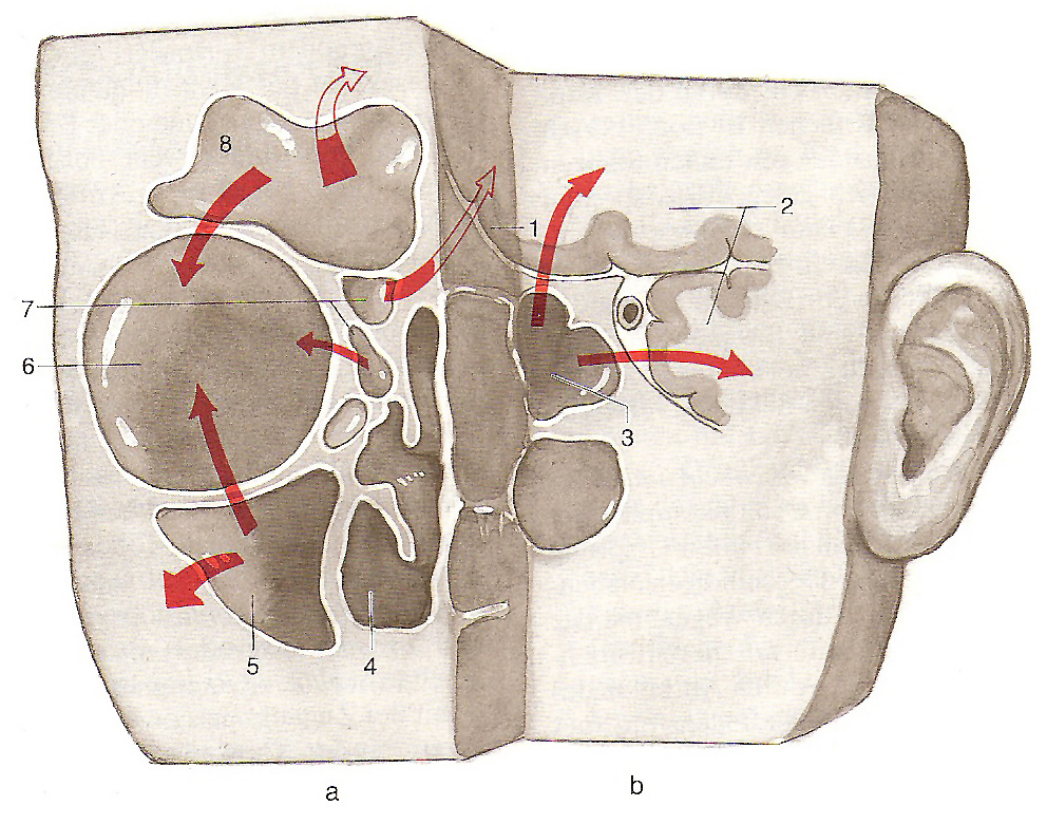

Abbildung 22: Mögliche Richtungen der Infektionsausbreitung bei Sinusitis (Quelle: Kastenbauer, Tardy jr. 1995; S.525)

a. Frontalschnitt in Höhe Stirnhöhle-Kieferhöhle-Siebbein

b. Frontalschnitt in Höhe Keilbeinhöhle

1. basaler Liquorraum

2. Gehirn

3. Keilbeinhöhle

4. Nasenhöhle

5. Kieferhöhle

6. Orbita

7. Siebbein

8. Stirnhöhle 


\subsubsection{Ergebnisse Sinus maxillaris}

Bei der Untersuchung der Erkrankungen des Sinus maxillaris wurde soweit möglich die Sinusinnenfläche befundet. Als krank gewertet wurden Veränderungen der Oberfläche wie zum Beispiel eine fein poröse Corticalis als Hinweis für eine Hyperämisierung der Schleimhäute zu Lebzeiten des Individuums. Auch Veränderungen wie poröse Platten, unphysiologisch auftretende Gefäßimpressionen oder reaktive Neubildungen nach chronischer Reizung des Periosts gelten als Zeichen einer Sinusitis.

Ein Individuum wurde als ,krank“ gewertet, wenn mindestens ein Sinus pathologische Veränderungen zeigte.

Eine postmortale Eröffnung oder Punktion der Sinus paranasales wurde nicht durchgeführt.

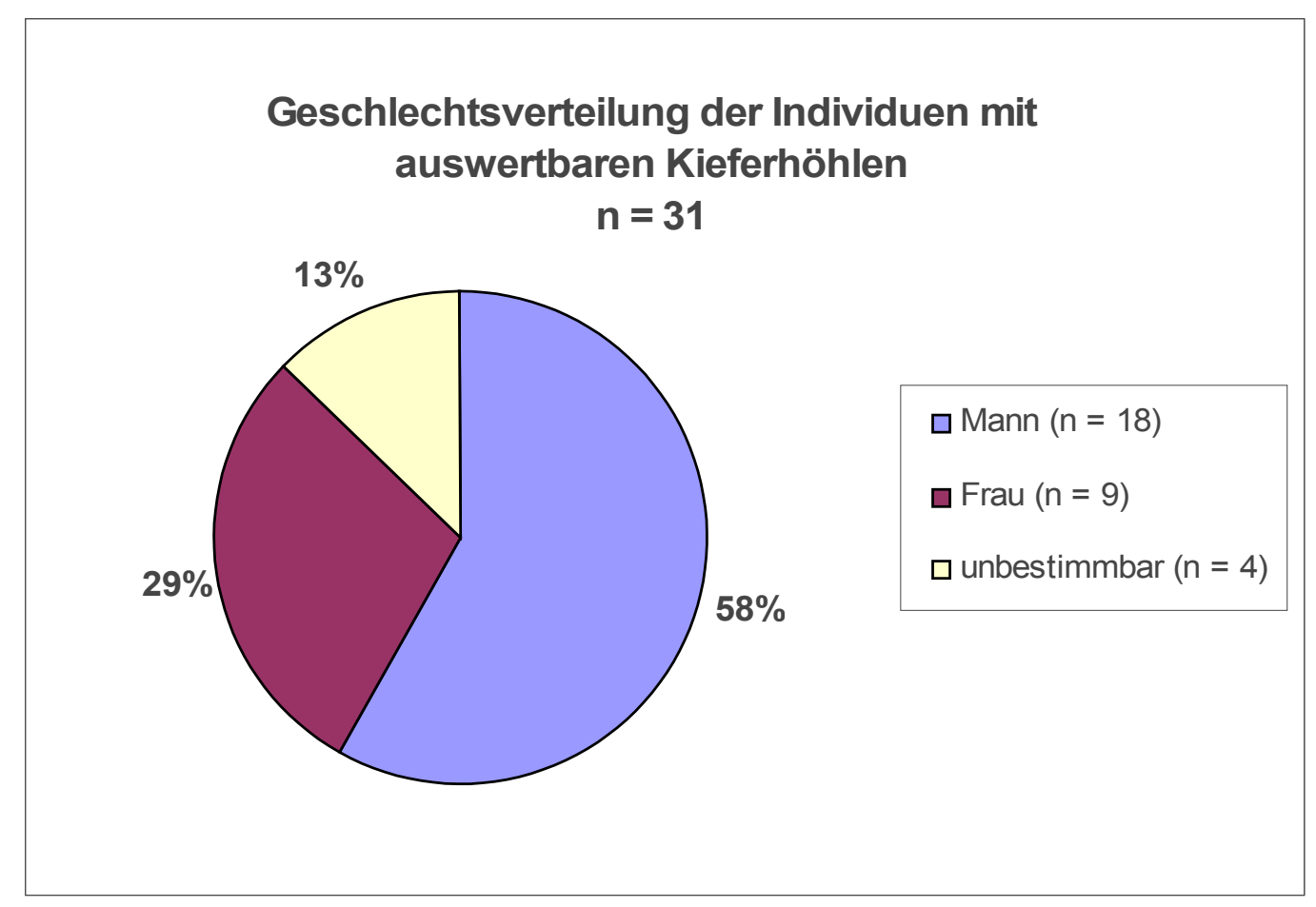

Abbildung 23: Geschlechtsverteilung der auswertbaren Kieferhöhlen 
Es können die Kieferhöhlen von 31 Individuen zur paläopathologischen Untersuchung verwendet werden. Die Geschlechtsverteilung der untersuchten Individuen mit Sinus maxillaris wird in Abbildung 23 graphisch wiedergegeben.

Alle Geschlechter sind von Erkrankungen der Kieferhöhle in unterschiedlichem Schweregrad stark betroffen (Abb. 24).

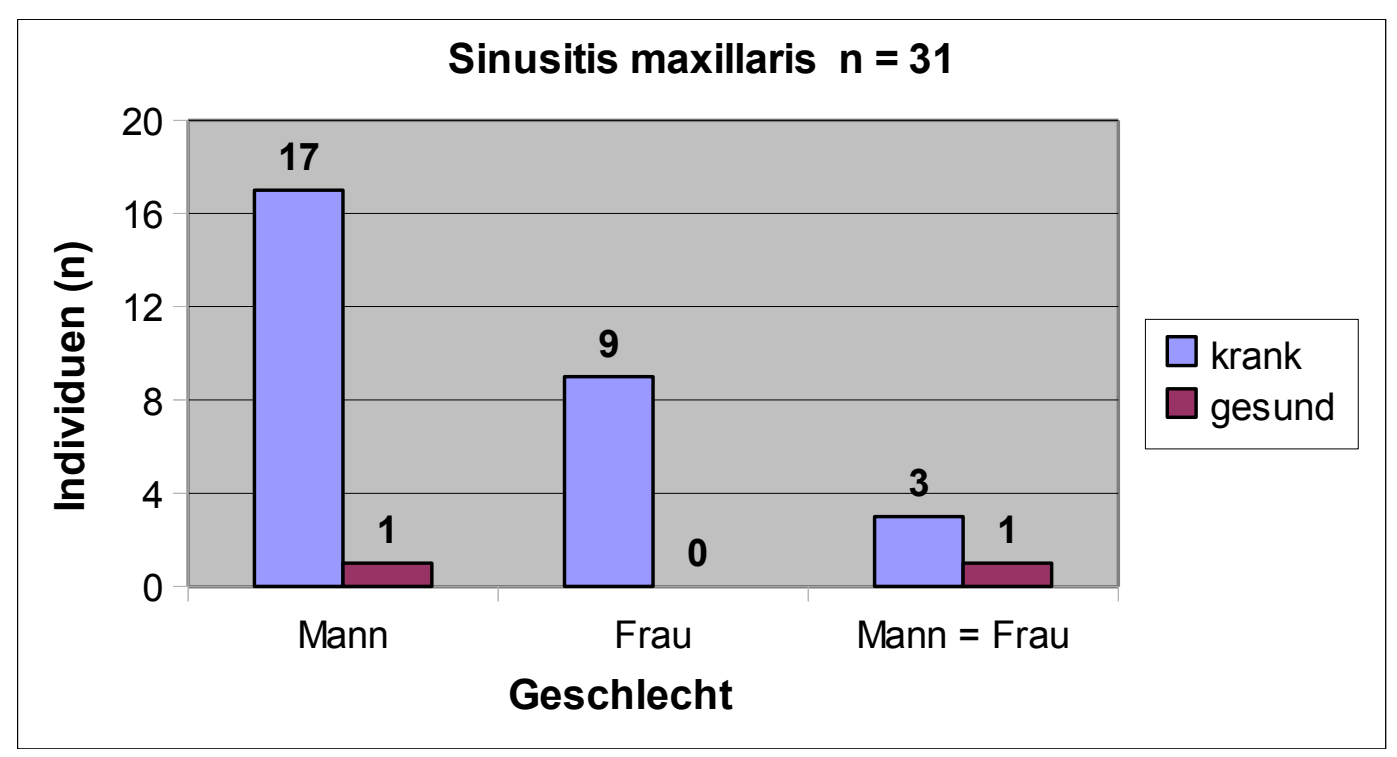

Abbildung 24: Geschlechtsspezifische Verteilung der Kieferhöhlenentzündungen

Es zeigt sich ein deutliches Überwiegen der erkrankten Kieferhöhlen: 94 Prozent der Männer und sogar 100 Prozent der Frauen weisen mindestens einen erkrankten Sinus maxillaris auf. Frei von pathologischen Veränderungen sind nur zwei der 31 Individuen. Allerdings sind in dieser Einteilung alle Ausprägungsgrade von pathologischen Läsionen enthalten. Im Diagramm ist ein Individuum mit feinen netzförmigen Gefäßimpressionen auf der Knochenoberfläche als Zeichen für eine chronisch hyperämische Schleimhaut bei seröser Rhinitis genauso krank wie ein Individuum mit eingeschmolzenen Fistelgängen nach massiv eitriger Sinusitis maxillaris. 
Als schwer pathologisch verändert zählten Kieferhöhlen, deren Oberfläche Entzündungszeichen wie reaktive Neubildungen oder Einschmelzungen aufwies. Dagegen wurden Kieferhöhlen mit nur schwach veränderter Oberfläche und lediglich vermehrten Gefäßimpressionen und poröser Corticalis als schwach pathologisch verändert angesehen.

Bei akut oder chronisch Erkrankten sind stärkere Krankheitszeichen zu erwarten als bei Individuen, die eine solche Infektion überstanden haben und deren Kieferhöhlen sich wieder organisieren. Aber auch zu Lebzeiten bereits gesunde und symptomfreie Individuen bieten bei der paäopathologischen Untersuchung noch sichtbare Spuren einer vergangenen Sinusitis wie vermehrte Gefäßimpressionen und nahezu vollständig integrierte Auflagerungen und werden als erkrankt gewertet.

Eine hyperämische Schleimhaut mit resultierenden vermehrten Gefäßimpressionen kann als Reaktion auf eine chronische Reizung durch Heuschnupfen, inhalative Noxen wie Qualm und Rauch oder nach Abheilung und Reorganisation schwerer Infektionen mit bleibender Hypervaskularisation entstehen. Vermehrte netzartige Gefäßimpressionen ohne Neubildungen oder Einschmelzungen als Zeichen einer ausgeprägten bakteriellen Infektion sind an der knöchernen Kieferhöhle nachweisbar. Individuen mit diesen Merkmalen stellen in der Population Baunach schwach ausgeprägte pathologische Veränderungen dar.

Im Gegenteil dazu gilt als schwer erkrankt eine durch bakterielle Superinfektion verursachte Sinusitis maxillaris. Bei akut oder chronisch Erkrankten sind stärkere Krankheitszeichen zu erwarten als bei Individuen, die eine solche Infektion überstanden haben und deren Kieferhöhlen sich wieder organisieren. Aber auch zu Lebzeiten bereits gesunde und symptomfreie Individuen bieten bei der paläopathologischen Untersuchung noch sichtbare Spuren einer vergangenen Sinusitis und werden als erkrankt gewertet. Im folgenden Diagramm (Abb. 25) erfolgt eine Differenzierung in leicht und schwer erkrankte Fälle. 


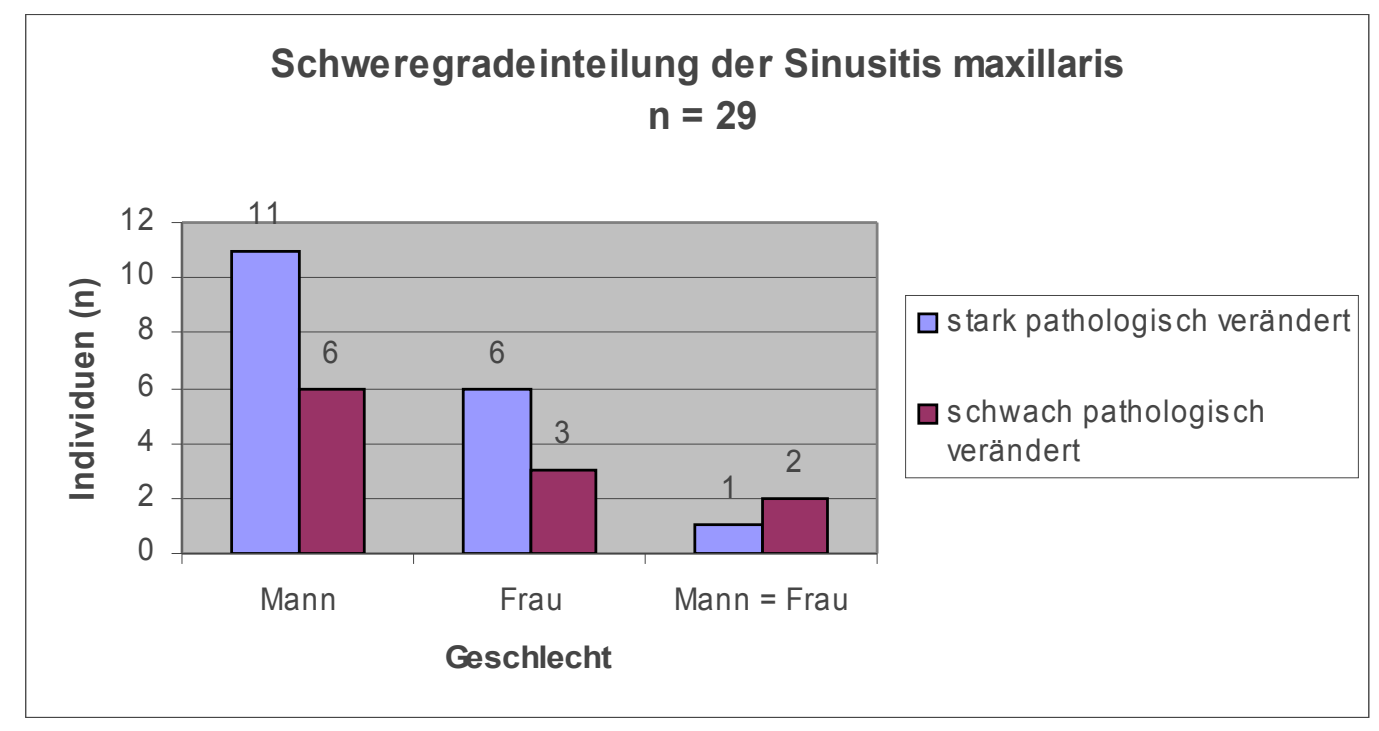

Abbildung 25: Einteilung nach Schweregrad der Kieferhöhlenentzündung

Hier zeigt sich, dass bei Männern (54 Prozent stark pathologisch verändert) und Frauen (50 Prozent stark pathologisch verändert) die stark pathologisch veränderten Kieferhöhlen überwiegen. In der die Kinder repräsentierenden Kategorie Mann $=$ Frau sind lediglich 33 Prozent als stark pathologisch veränderte Sinus maxillares zu finden.

\subsubsection{Ergebnisse Sinus frontalis}

Für eine Sinusitis frontalis gelten ähnliche Risikofaktoren und Komplikationsmöglichkeiten wie für eine Sinusitis maxillaris. Allerdings schafft die enge räumliche Nähe zum Gehirn mehr intracranielle Komplikationen (Abb. 26). Die Möglichkeiten für die Entstehung einer Meningitis, Enzephalitis oder Sinusthrombose und ihre Konsequenzen für die Gesundheit eines Individuums wurden bereits bei der Sinusitis maxillaris genannt. 


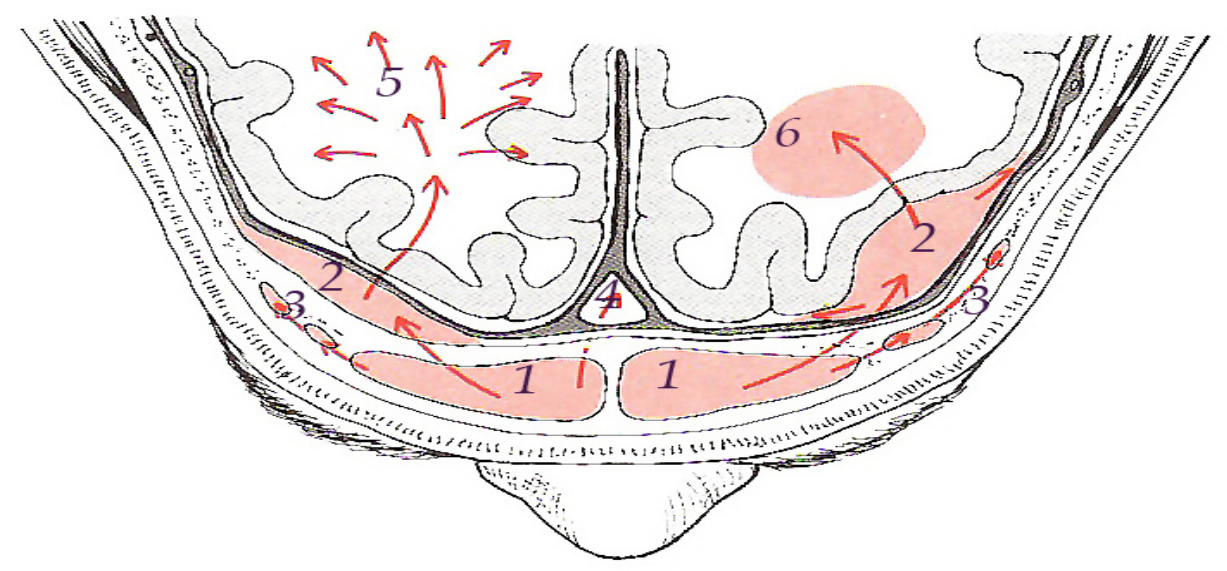

Abbildung 26: Endocranielle Komplikationsmöglichkeiten bei Sinusitis frontalis (Quelle: Kastenbauer, Tardy jr. 1995; S.527)

1. Sinusitis frontalis

2. epiduraler Abszess rechts, subduraler Abszess links

3. Ostitis

4. Thrombose des Sinus sagittalis superior

5. Enzephalitis

6. Hirnabszess

Es konnten für die Untersuchung alle die Individuen berücksichtigt werden, bei denen mindestens eine Stirnhöhle eröffnet war und deren Zustand eine Befundung zuließ. Bei vielen Schädeln war die Stirnhöhle nicht einsehbar, ohne den komplett erhaltenen Schädel zu zerstören. Daraus erklärt sich auch der relativ geringe Stichprobenumfang (Abb. 27). 


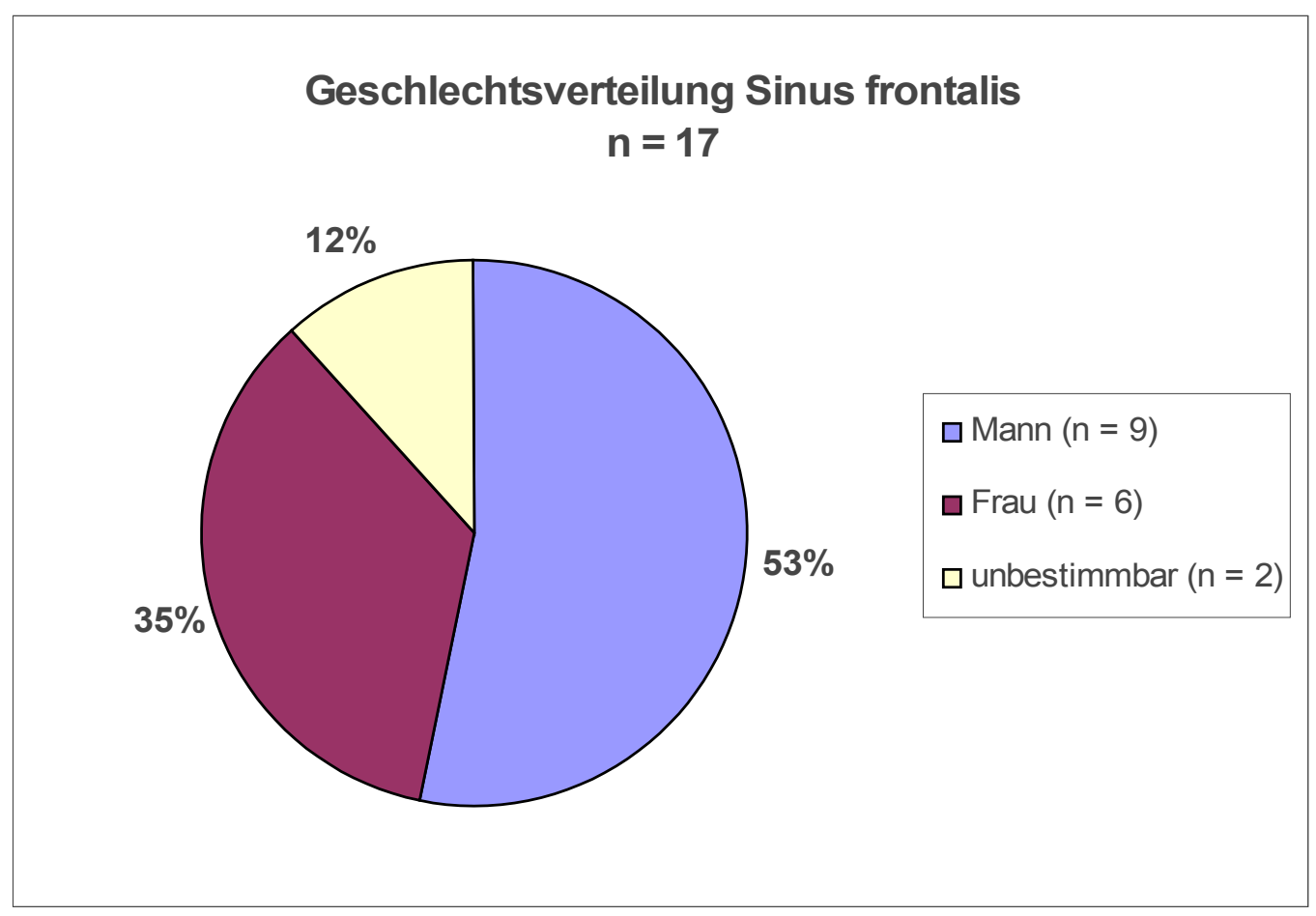

Abbildung 27: Geschlechtsverteilung der Individuen mit auswertbaren Stirnhöhlen

Die Geschlechtsverteilung entspricht den bisherigen Verteilungen bei Sinusitis maxillaris und Rhinitis. Bei der Inzidenz der Sinusitis frontalis ergeben sich für die Baunacher Individuen folgende Ergebnisse (Abb. 28):

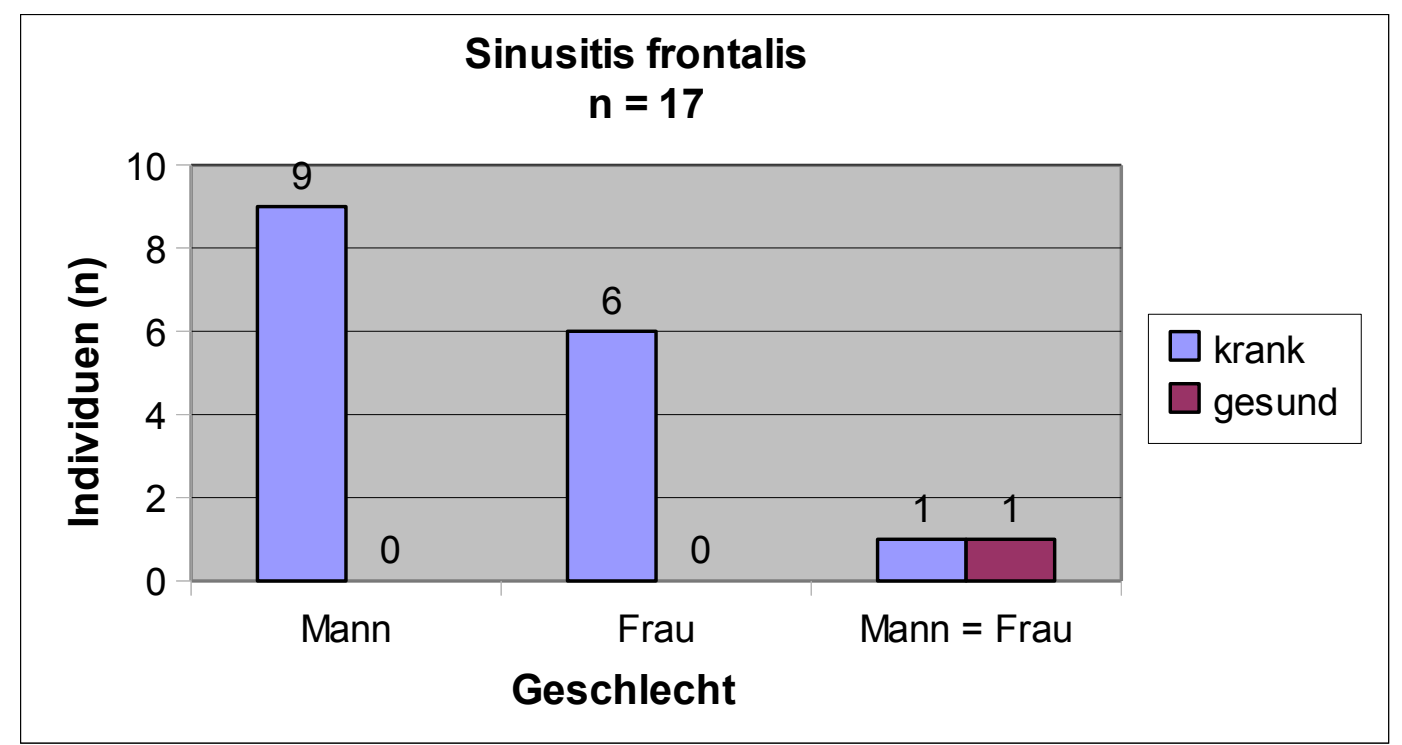

Abbildung 28: Geschlechtsspezifische Verteilung der Stirnhöhlenentzündung 


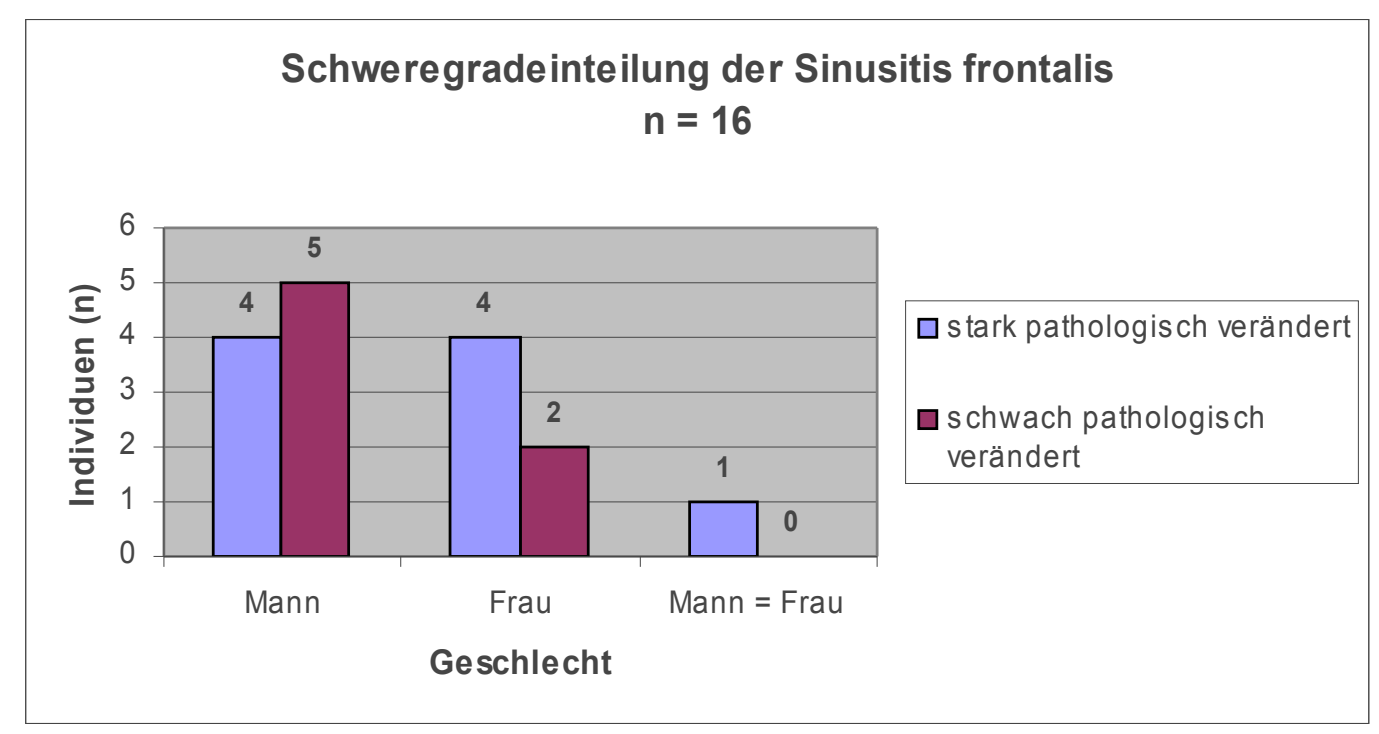

Abbildung 29: Einteilung nach Schweregrad der Stirnhöhlenentzündung

Die Einteilung in Schweregrade der pathologisch veränderten Stirnhöhlen zeigt in Abbildung 29 und 30, dass sich bei den Männern nur etwa jede zweite Stirnhöhle (44 Prozent) in einem stark pathologisch veränderten Zustand befand. Zeichen schwacher pathologischer Veränderungen, beispielsweise bei einer Schleimhautreizung oder bei sich im Zustand der Organisation befindlichen erkrankten Knochenoberfächen, sind bei 56 Prozent der Männer in Baunach zu finden.

Bei den Frauen stellt sich eine andere Situation dar. Hier finden sich 67 Prozent mit stark pathologisch veränderter Oberfläche. Nur ein Drittel der erkrankten Frauen befindet sich im Heilungsstadium oder zeigt Zeichen einer Schleimhautreizung. 


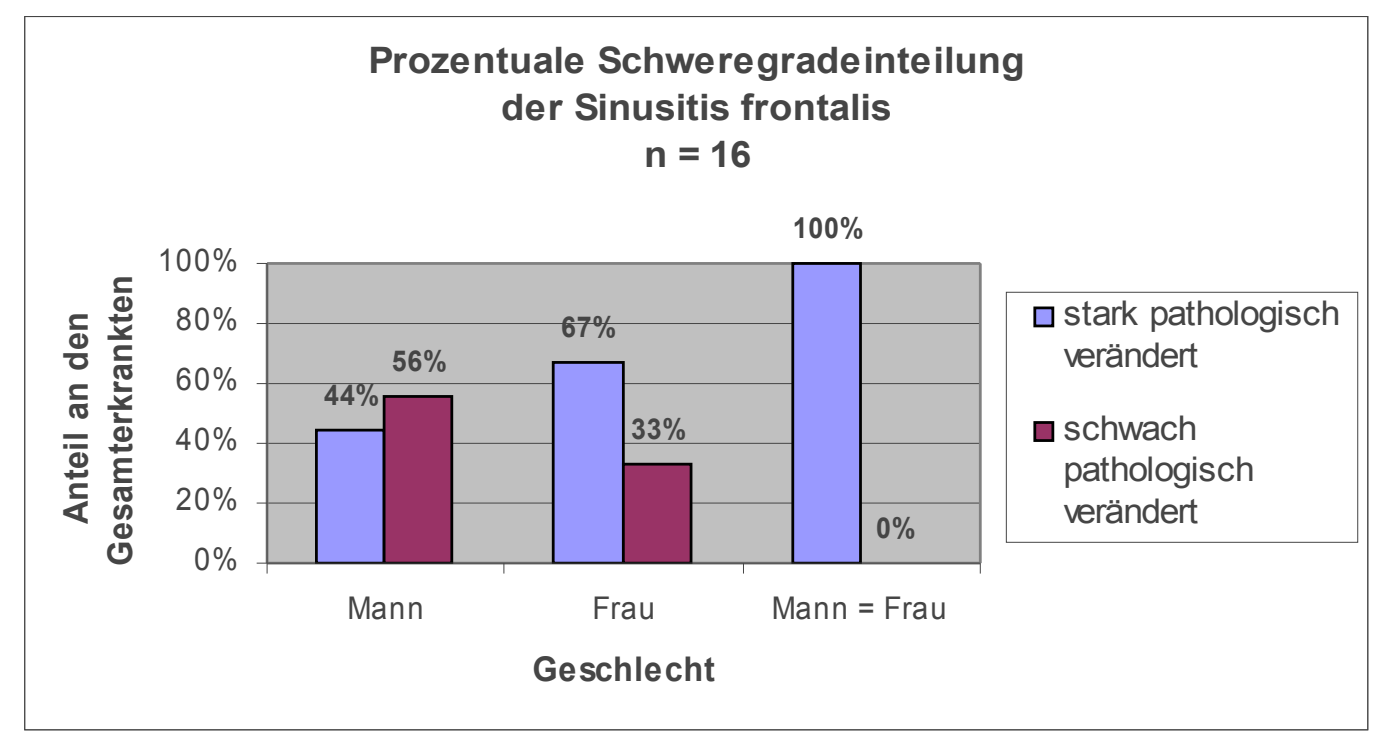

Abbildung 30: Prozentuale Schweregradeinteilung bei Stirnhöhlenentzündung

Anders als bei den Kieferhöhlenerkrankungen findet man bei den Männer keinen deutlich höheren Anteil an schwach erkrankten Stirnhöhlen. Bei den Frauen gleichen sich die Verteilungen von stark und schwach pathologisch veränderten Oberflächen. Die Verteilung bei Mann = Frau ist bei einer Stichprobenzahl von lediglich 2 Individuen und einem erkrankten Sinus frontalis nicht aussagekräftig.

\subsection{Pathologische Befunde im Mittelohr}

\subsubsection{Anatomische und physiologische Grundlagen}

Das Ohr ist Sitz der Reizaufnahme für den Hör- und den Gleichgewichtssinn. Um diese Aufgabe geschützt vor Traumata und anderen Gefahren wahrzunehmen, befinden sich alle wichtigen Strukturen dieser beiden Sinne in einem der härtesten Knochen des menschlichen Körpers, der Pars petrosa (dt.: Felsenbein) des Os temporale. 
Das Ohr gliedert sich in 3 Abschnitte: 1. äußeres Ohr mit Gehörgang

2. Mittelohr

3. Innenohr.

Der äußere Gehörgang befindet sich zum größten Teil im Os temporale und ist zudem überwiegend von knorpeliger Struktur und mit verhornender Epidermis ausgekleidet. Ein kurzer knöcherner Gang, der Meatus acusticus externus, reicht in das Os temporale und begrenzt abschließend das Mittelohr. Dieser Teil dient vor allem der Schallleitung.

Das Trommelfell trennt den äußeren Gehörgang vom Mittelohr. Hier findet eine Schallverstärkung um den Faktor von circa 20 statt. Dies wird erreicht, indem die Druckimpulse der Schallwellen von einer relativ großen Membran (Trommelfell) aufgenommen werden und durch die Weiterleitung über die drei Gehörknöchelchen Hammer, Amboss und Steigbügel auf eine viel kleinere Membran (ovales Fenster) weitergeleitet werden. Letztlich überträgt der Steigbügel mechanisch die Schallwellen am ovalen Fenster auf einen flüssigkeitsgefüllten Raum im Innenohr. Aus Gründen des Druckausgleichs ist das Mittelohr mit dem Rachenraum über einen Kanal, die Eustachische Röhre oder Tuba auditiva, verbunden. Das Mittelohr ist ebenso wie die pneumatisierten Bereiche des Schläfenbeins mit Schleimhaut ausgekleidet. Die Druckwellen werden hier auf das Labyrinthus cochlearis (Hörorgan) und das Labyrinthus vestibularis (Gleichgewichtsorgan) weitergeleitet. In diesen äußerst geschützt liegenden Systemen findet dann die neuronale Codierung der empfangenen Impulse statt.

\subsubsection{Erkrankungen des Mittelohrs}

Das Mittelohr ist wesentlich für das Hören, das benachbarte Innenohr verantwortlich für den Gleichgewichtssinn. Erkrankungen in diesem Bereich können diese also stark beeinflussen. Bedingt durch die Lage des Mittelohrs und die damit verbundene Nähe zum Gehirn und bedeutenden Nerven und Gefäßen kann eine solche Erkrankung große Auswirkungen auf das betreffende Individuum haben. 
Eine Entzündung des Mittelohrs wird als Otitis media bezeichnet. Zumeist wird diese durch über die eustachische Röhre aus dem Pharynxs aufsteigenden Keime verursacht (Abbildung 31). Häufige Erreger einer akuten Mittelohrentzündung in aktuellen Studien sind gegenwärtig tubogen eingewanderte Krankheitserreger aus dem Nasen-Rachen-Raum und den tieferen Atemwegen wie Streptoccocus pneumonia (NUSSINOVITCH et al. 2004) oder andere Strepptokokkenstämme, sowie Haemophilus influenza oder Staphylokokken (NAUMANN, SCHERER 1998). Auch virale Infektionen mit konsekutiver bakterieller Superinfektion sind möglich. Diese Bakterien neigen zur Eiterbildung und Einschmelzung der begrenzenden Strukturen des Mittelohrs. Man kann davon ausgehen, dass ähnliche bzw. die gleichen Keime schon unsere Vorfahren plagten. Vor der Einführung der Antibiotikatherapie war eine Otitis media aufgrund der Nachbarschaft wichtiger Strukturen sehr häufig mit lebensbedrohlichen Komplikationen behaftet. Unter Einsatz moderner Therapie und Pharmaka konnte die Komplikationsrate in westeuropäischen Ländern von 17 Prozent auf 1 Prozent gesenkt (PREYER 2004). Daher ist die Prävalenz einer eitrigen Otitis media mit ihren bedrohlichen Komplikationen in heutiger Zeit niedriger als in paläopathologisch untersuchten Populationen.

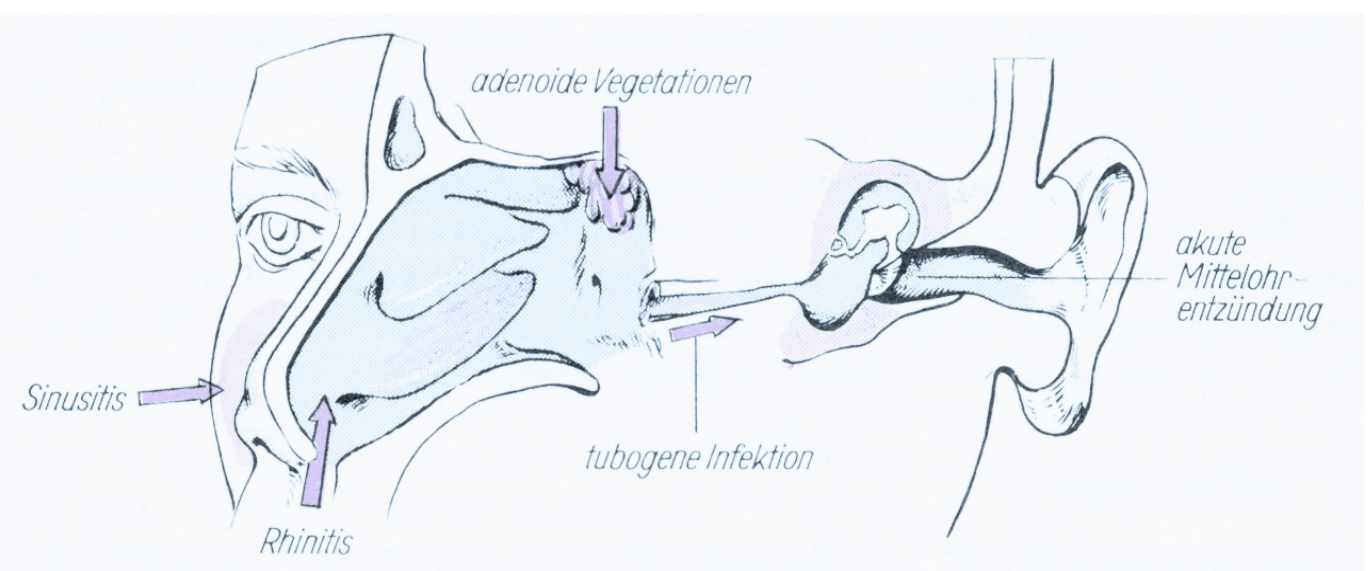

Abbildung 31: schematische Darstellung möglicher Infektionswege einer Otitis media (Quelle: Helms, Jahrsdorfer 1996;S.258) 
Eine eitrige Otitis media kann auch bei intakter Immunabwehr ohne antibiotische Therapie heilen. Dabei perforiert das Trommelfell, der Eiter kann abfließen und das Mittelohr vernarbt. Schallleitungsschwerhörigkeit ist eine häufige Folge.

Die Mittelohrentzündung kann aber auch in ein chronisches Stadium übergehen. Dies ist seltener als eine akute Mittelohrentzündung und beruht auf einer eitrigen Ostitis oder Mukositis im Bereich des Mittelohres. Meist wird in diesem chronischen Stadium kein Schmerz empfunden. Neu aufgetretene Schmerzen im Zusammenhang mit chronischem Eiterfluss aus dem Mittelohr sind ein Hinweis auf eine sich entwickelnde Komplikation.

Diese werden topografisch in extracraniell und endocraniell unterschieden. Lebensbedrohlich sind zumeist die endocraniellen, die sich in der Schädelhöhle abspielen und häufig zu einer weiteren Infektionsausbreitung führen.

Die folgende Tabelle 3 sowie die Abbildung 32 verdeutlichen mögliche Komplikationen sowie eventuelle Ausbreitungsrichtungen einer Otitis media.

\begin{tabular}{|l|l|}
\hline \multicolumn{1}{|c|}{ Extracranielle Komplikationen } & \multicolumn{1}{|c|}{ Intracranielle Komplikationen } \\
\hline Otitis externa & Meningitis \\
Labyrinthitis & Epiduralabszess \\
Fazialisparese & Subduralempyem \\
Chronische Otitis media & Hirnabszess \\
Petrositis & Sinusvenenthrombose \\
Mastoiditis & Otogener Hydrocephalus \\
\hline
\end{tabular}

Tabelle 3: Komplikationen der Otitis media

Es wird also deutlich, was für eine Beeinträchtigung des Alltags oder sogar Lebensgefahr sich aus einer Otitis media ergeben kann, die nicht durch spontane Trommelfellperforation zur Abheilung gelangt. 
Sämtliche endocranielle Komplikationsmöglichkeiten können bei ausbleibender Behandlung zum Tod des Kranken führen. Auch im Zeitalter der Antibiotika-Therapie ist häufig die chirurgische Therapie zur Abzessentlastung bei Eiterherden unumgänglich.

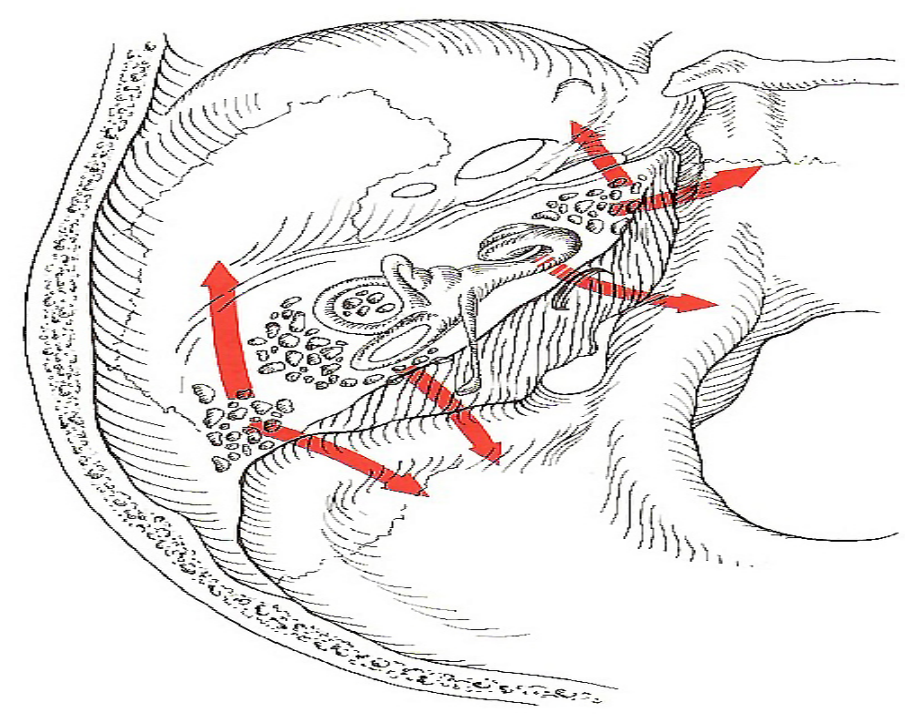

Abbildung 32: mögliche intracranielle Ausbreitungsrichtungen bei Otitis media (Quelle: Helms, Jahrsdorfer 1996; S.263)

\subsubsection{Ergebnisse Mittelohr}

Bei der Untersuchung der Mittelohren der Baunacher Skelete konnten 39 Individuen berücksichtigt werden. Ein Individuum muss durch mindestens 1 gut erhaltenes Mittelohr repräsentiert sein, um in die Wertung zu gelangen.

Endoskopisch untersucht wurde das Promontorium. Dies ist bei gesunden Personen annähernd oval und zeigt eine glatte Oberfläche. Ein Individuum galt als krank, wenn mindestens an einem Mittelohr krankhafte Veränderungen im Sinne von Einschmelzungen, zapfen- oder dornartige Neubildungen, alte organisierte plattenartige Neubildungen oder überdurchschnittlich starke Gefäßimpressionen bei der endoskopischen Untersuchung zu sehen waren. All diese Veränderungen 
gehen in der Regel auf entzündliche Vorgänge am Knochen zurück und repräsentieren somit sehr wahrscheinlich Spuren einer akuten oder sehr wahrscheinlich chronischen Otitis media bzw. deren Abheilungszustand.

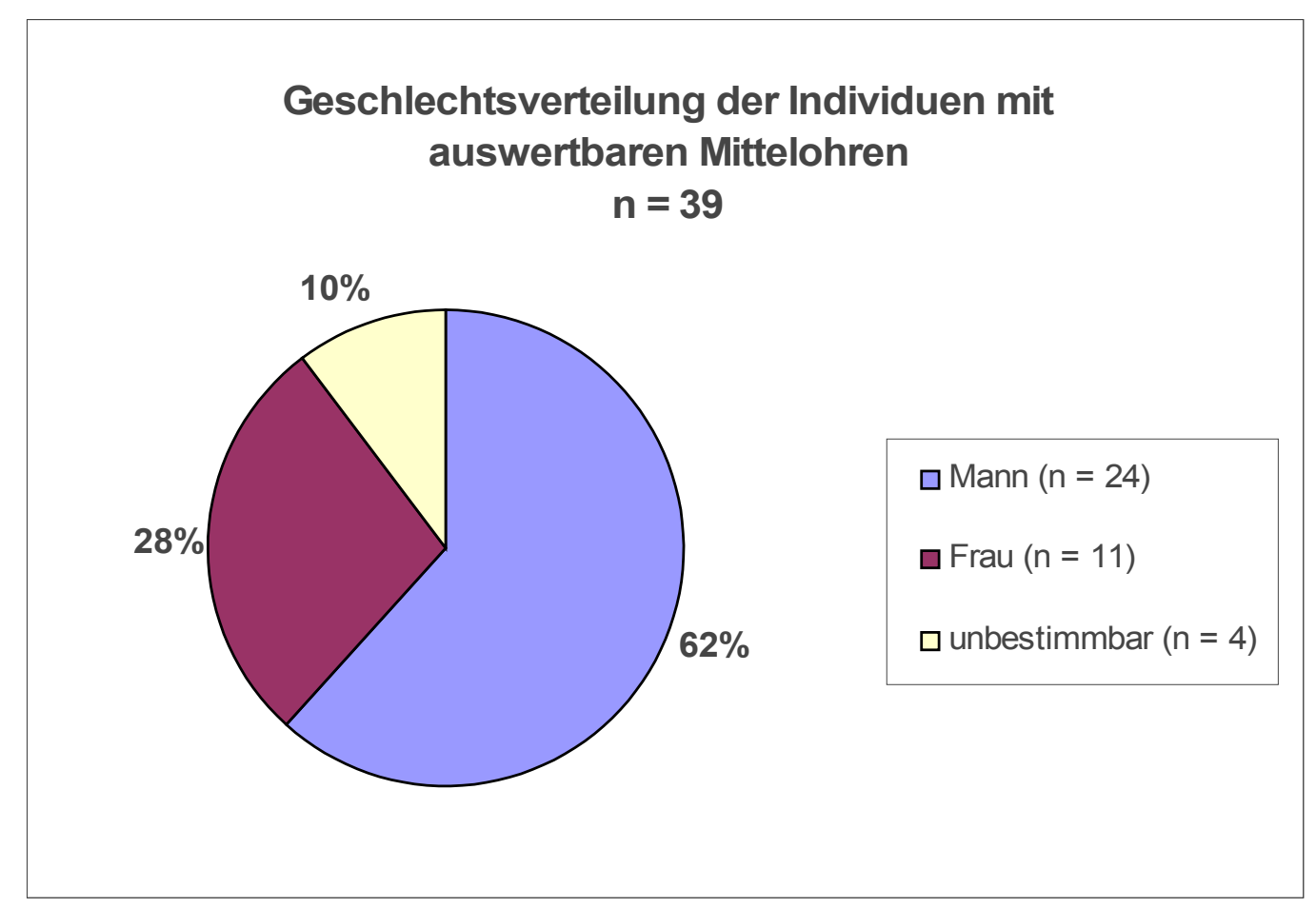

Abbildung 33: Geschlechtsverteilung der Mittelohren

Die Abbildung 33 weist eine deutliche Dominanz männlicher Individuen mit verwertbaren Mittelohren auf.

Bei einer Einteilung der Geschlechter in "gesundes Mittelohr" und "krankes Mittelohr“" ergeben sich dabei folgende Verteilungen: 


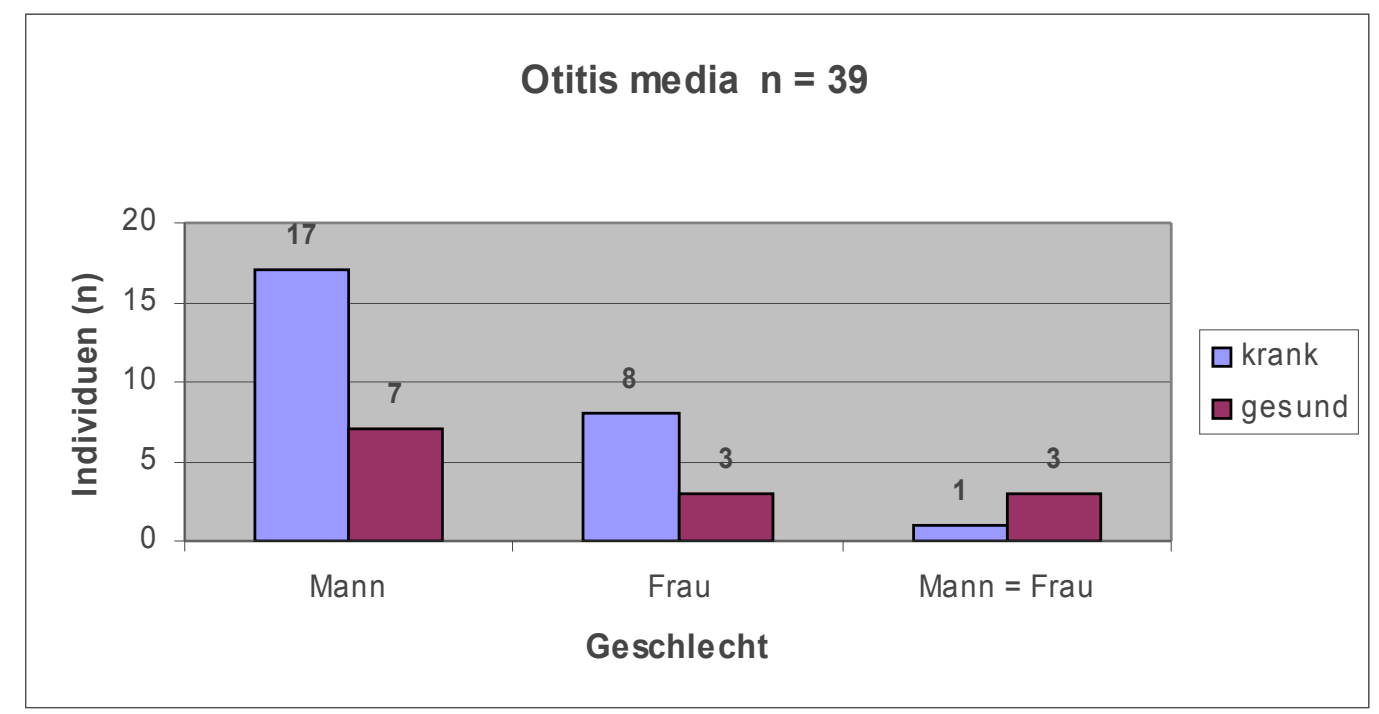

Abbildung 34: Geschlechtsspezifische Verteilung der Mittelohrentzündung

Bei der prozentualen Verteilung innerhalb der Geschlechter zeigen sich ausgeglichene Verteilungsverhältnisse bei Mann und Frau. Grafisch werden die Fallzahlen sowie die prozentuale Verteilung in den Abbildungen 34 und 35 dargestellt.

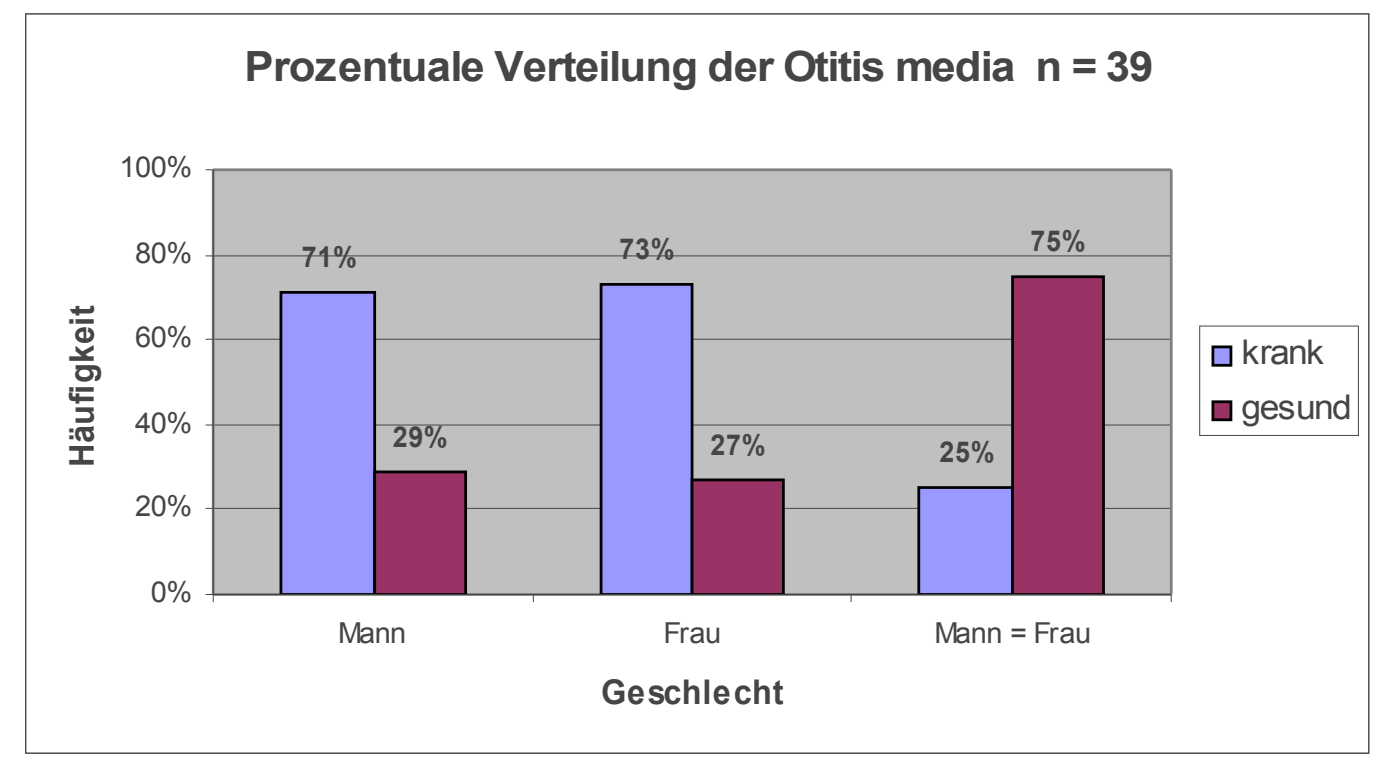

Abbildung 35: Prozentuale Verteilung der Mittelohrentzündung 


\subsection{Pathologische Befunde im Mastoid}

\subsubsection{Anatomische und physiologische Grundlagen}

Der Processus mastoideus (dt. Warzenfortsatz) dient als knöcherner Ansatz des den Kopf bewegenden Musculus sternocleidomastoideus. Die Ausprägung des Warzenfortsatzes hängt somit von der individuellen körperlichen Konstitution sowie vom Geschlecht ab. Aber auch der Pneumatisationsgrad ist für die Größe und Ausprägung des Processus mastoideus von Bedeutung.

Die bei der Geburt ursprünglich bestehende Kompaktheit der Spongiosa des Processus mastoideus wird im Verlaufe des Wachstums durch das Einsprossen von Epithelknospen aus dem Bereich des Mittelohres aufgehoben. Es bilden sich pneumatische Räume, ausgehend von dem schon beim Neugeborenen vorhandenem Antrum. Dieser Prozess sollte mit dem Erreichen des sechsten Lebensjahres abgeschlossen sein und kann fast alle Abschnitte des Os temporale einschließen (MARX 1939). Durch frühkindliche Mittelohrentzündungen mit Erkrankung der aussprossenden Schleimhaut kann eine Pneumatisationshemmung eintreten. Der sonst pneumatisierte Bereich des Os temporale bleibt dann spongiös strukturiert (WITTMAACK 1918). Aber auch eine Entzündung des Mittelohres nach abgeschlossener Pneumatisation kann durch osteoklastische und osteoblastische Vorgänge zu einem spongiösen Aufbau des Processus mastoideus führen.

\subsubsection{Erkrankungen des Mastoids}

Laut WERNER und LIPPERT (2003) geht jede Otitis media mit einer Begleitentzündung des Warzenfortsatzes einher. Eine Mastoiditis stellt also unter allen otogen entzündlichen Komplikationen einer Otitis media die häufigste Begleitentzündung dar. Die vermutliche Ursache dieser Entzündung ist eine Verlegung des Ausgangs aus dem Antrum mastoideum der pneumatischen Zellen in die Cavitas tympani durch entzündlich geschwollene Schleimhaut und/oder Eiter. Dies führt zu einem Abflusshindernis von entzündlichem Sekret und Eiter aus den pneumatisierten Zellen des Warzenfortsatzes und schließlich zu einer Einschmelzung der 
knöchernen Septen. Es bildet sich somit aus dem initialen Sekretverhalt ein Empyem im Os temporale (MARX 1939). Im späteren Krankheitsverlauf ist auch ein Durchbruch durch die Corticalis möglich, bei der sich der im Mastoid gebildete Eiter drainiert. Die Drainagerichtung ist entscheidend für den weiteren Krankheitsverlauf. Bricht der Eiter an der Spitze des Warzenfortsatzes nach außen, entsteht ein subperiostaler Abszess, auch Bezold-Mastoiditis genannt. Dieser Abszess schmilzt entlang der Faszie des M. sternocleidomastoideus bis zum Ursprung des Muskels ein und entleert sich dort häufig über die Haut. Die akute und schmerzhafte Erkrankung wäre in diesem Falle zunächst überwunden, da der Abszess nun eine Drainage zur Körperoberfläche gebildet hat und somit eine Abszessentlastung gegeben ist. Eine chronische Fistel ist häufig die Folge.

Allerdings kann sich eine Mastoiditis auch endocraniell drainieren. Dort kann kein Eiter abfließen und die Auswirkungen dieser endocraniellen Komplikation einer Mastoiditis stellen somit ohne chirurgische Intervention eine lebensbedrohliche Situation dar. Die folgende Grafik (Abbildung 36) gibt einen Überblick über mögliche Drainagerichtungen:

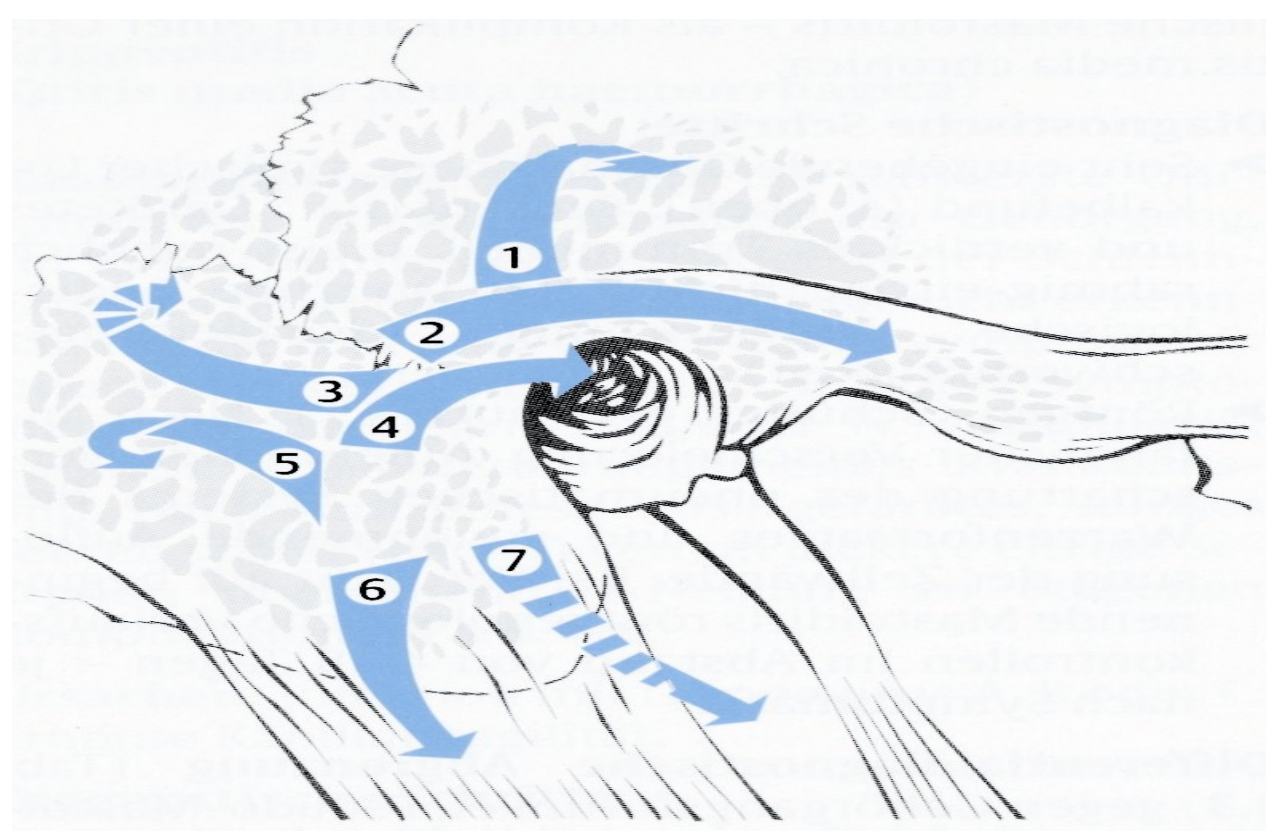

Abbildung 36: Mögliche Abszessentlastungsrichtungen bei Mastoiditis

(Quelle: Helms, Jahrsdorfer 1996; S.268) 
1. Richtung Squama temporalis und mittlere Schädelgrube

2. Richtung Jochbogen (Zygomatizitis)

3. Richtung Sinus sigmoideus und hintere Schädelgrube

4. Richtung äußere Gehörgang

5. Richtung Planum mastoideum (Subperiostaler Abszess)

6. Richtung Mastoidspitze und Ansatz M. sternocleidomastoideus (Bezold'scher Senkungsabszess)

7. Richtung mediale Fläche des Mastoids (Mouret'sche Mastoiditis, Parapharyngealabszess)

\subsubsection{Ergebnisse Mastoid}

Bei der Untersuchung der Mastoide erfolgte eine Präparation aller vorhandenen Warzenfortsätze. Dazu wurde die Spitze des Mastoids dorso-medial eröffnet. Im Regelfall befinden sich dort die etwa pfefferkorngroßen pneumatischen Zellen mit glatter Knochenoberfläche. Bei zu harter Corticalis, die einer schonenden Öffnung widerstand, wurden die Schläfenbeine geröntgt. Allerdings lässt sich im Röntgenbild nur der Pneumatisationszustand und größere Einschmelzungen bestimmen. Feinere Knochenoberflächenveränderungen auf den Septen der Cellulae mastoideae können so nicht beurteilt werden.

Von den Individuen der Population Baunach weisen 38 mindestens ein erhaltenes und für eine Befundung geeignetes Mastoid auf. In der Abbildung 37 wird die Geschlechtsverteilung der Individuen mit mindestens einem erhaltenen Mastoid dargestellt.

Ein Individuum zählt als krank, wenn mindestens ein Mastoid pathologische Veränderungen in den Cellulae mastoideae aufweist. 


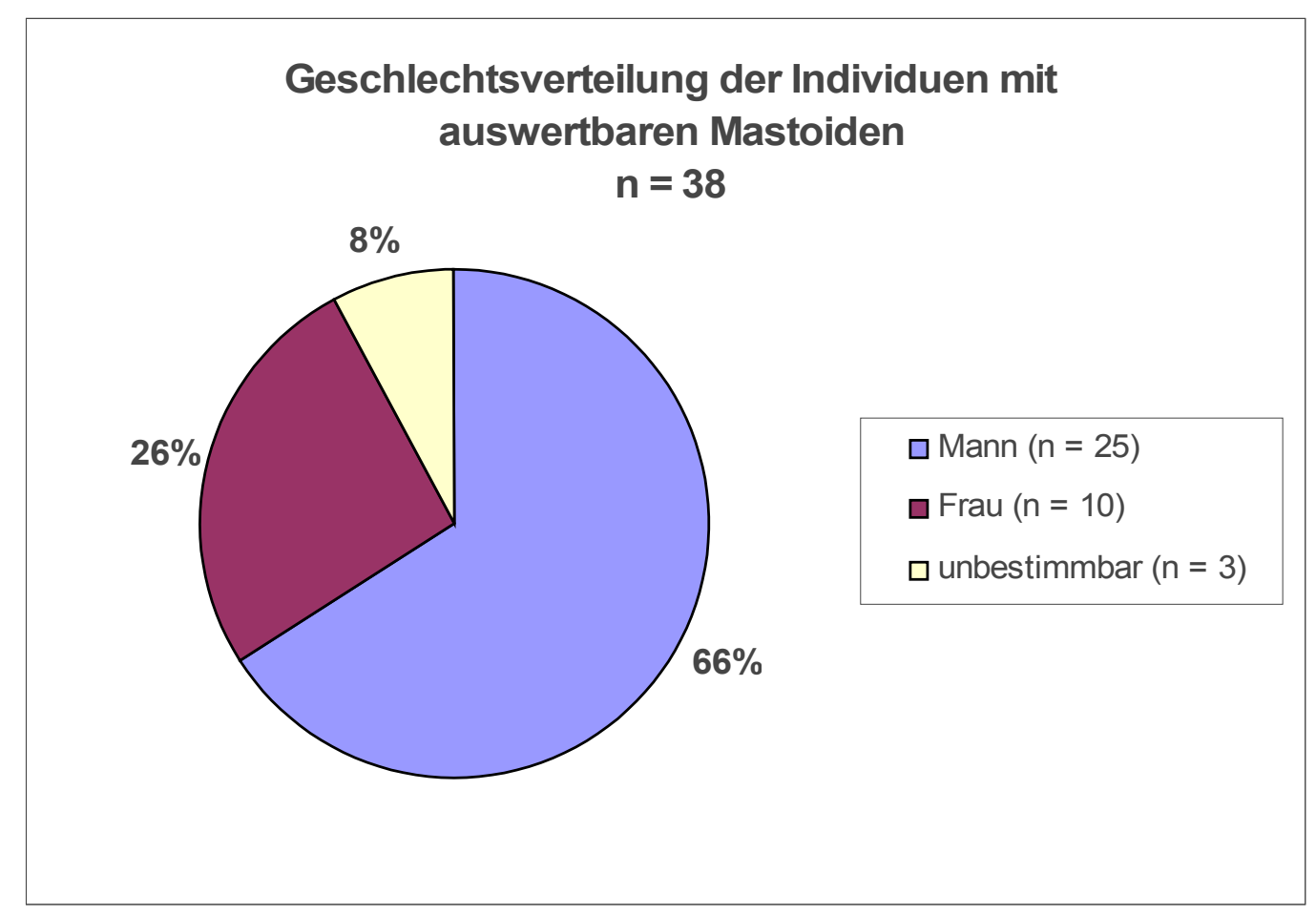

Abbildung 37: Geschlechtsverteilung der auswertbaren Mastoide

Aus der Gesamtmenge zeigen 21 Individuen, also 55 Prozent, Zeichen einer Mastoiditis. Zum Teil finden sich spitze nadelartige Neubildungen in etwa haselnussgroßen eingeschmolzenen Zellen als Zeichen für einen entzündlichen Vorgang mit Einschmelzungen und reaktiven ungeordneten Knochenneubildungen. Es sind aber auch Veränderungen im Sinne einer abgeheilten und in Organisation befindlichen Mastoiditis wie wulstartige Neubildungen oder netzförmige Gefäßimpressionenn auf den Wänden der Cellulae mastoideae zu finden. 


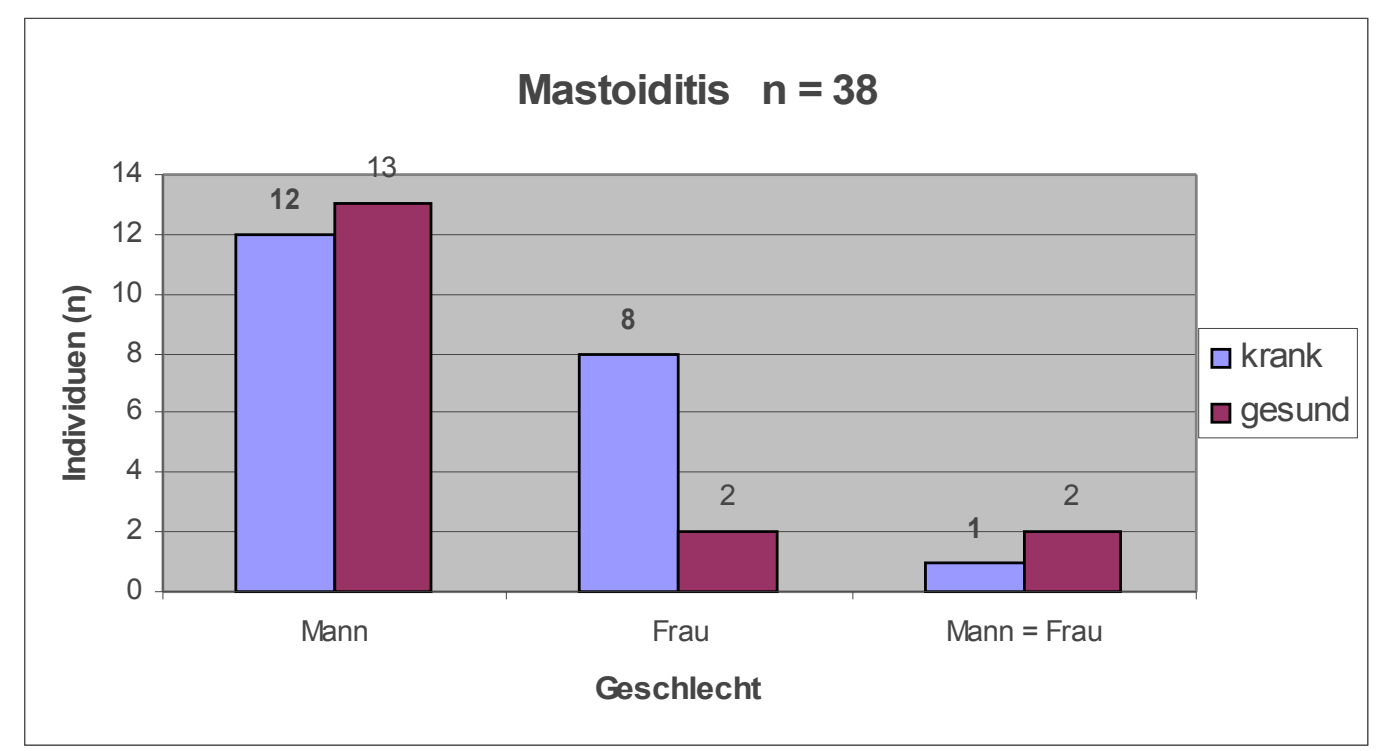

Abbildung 38: Geschlechtsspezifische Verteilung der Warzenfortsatzentzündung

Der Anteil an kranken und gesunden Individuen wird in Abbildung 38 deutlich.

Dabei zeigt sich, dass Frauen deutlich häufiger kranke Mastoide als Männer besitzen. In Abbildung 39 ist diese Verteilung graphisch dargestellt.

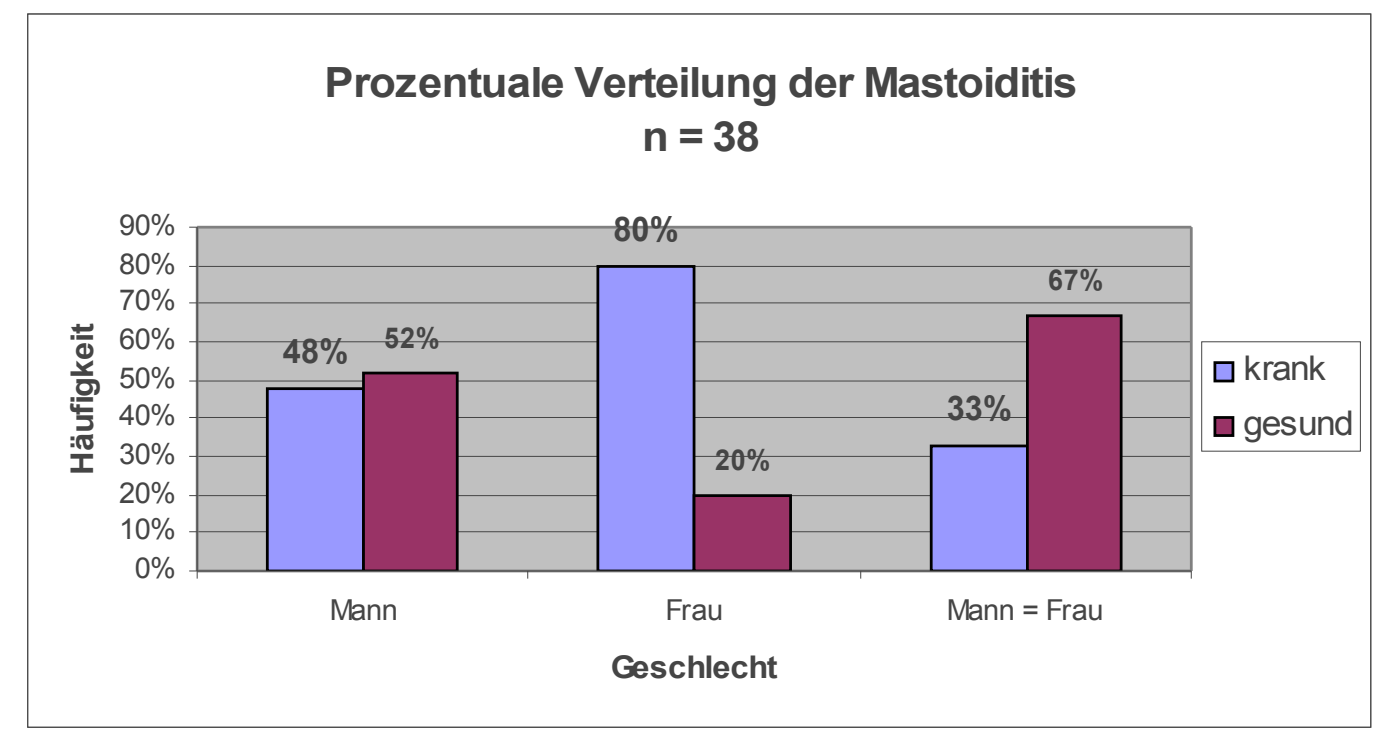

Abbildung 39: Prozentuale Verteilung der Warzenfortsatzentzündung 
Die Mastoide der Gruppe Mann = Frau zeigen nur zu einem Drittel pathologische Veränderungen. Ein ähnliches Verhältnis findet sich auch bei der prozentualen Verteilung der Otitis media in dieser Gruppe. Bei den Schädeln aus Baunach gehören zu dieser Gruppe meistens Kleinkinder und Jugendliche.

Eine chronische Otitis media in frühem Alter führt zu einer Pneumatisationshemmung des Mastoids. In der Regel nimmt diese Erkrankung ihren Ursprung in einer Minderbelüftung des Mittelohrs bei chronischer Tubenfunktionsstörung (WERNER, LIPPERT 2003).

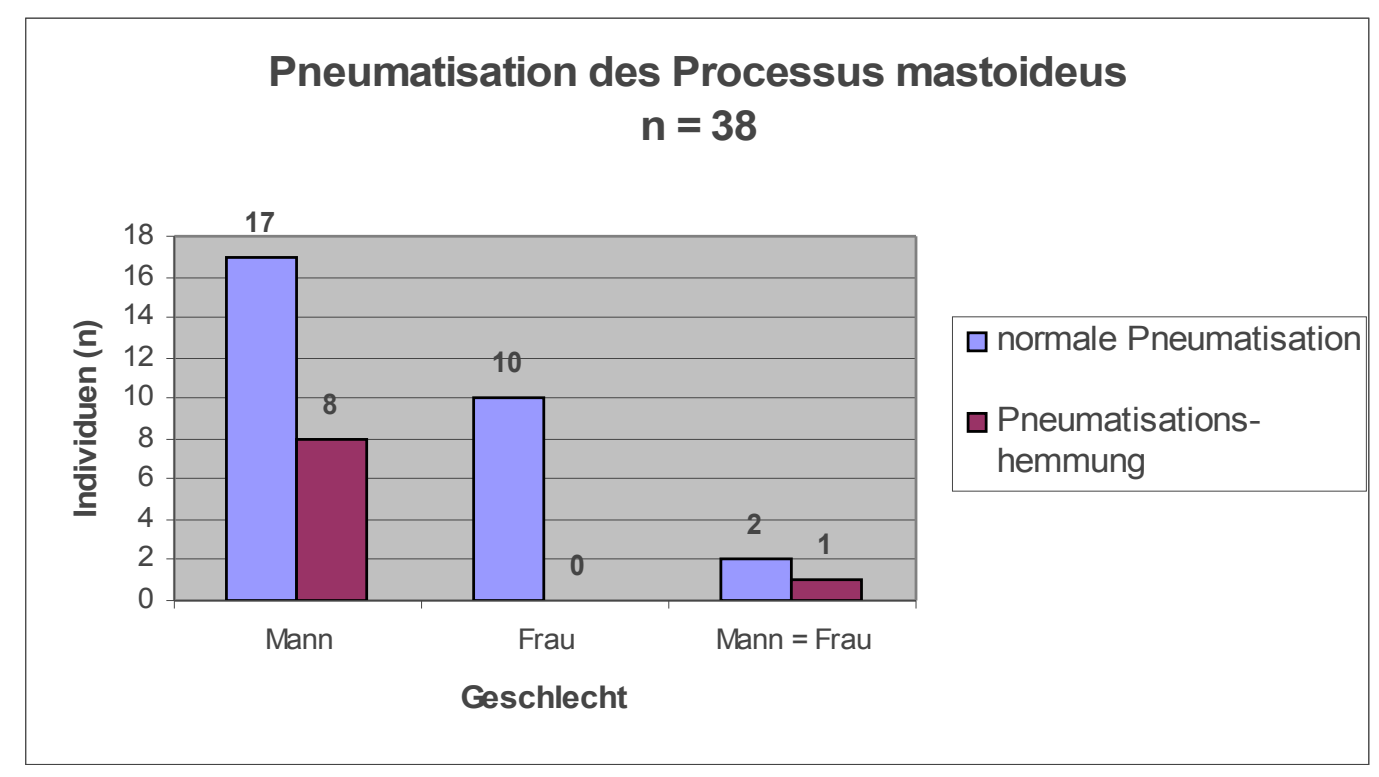

Abbildung 40: Pneumatisation des Warzenfortsatzes

Von den 38 Individuen, welche durch mindestens ein Mastoid repräsentiert sind, zeigen 23,7 Prozent eine Pneumatisationshemmung beziehungsweise ein komplett spongiöses Mastoid. Es ist somit anzunehmen, dass wenigstens ein Viertel der Baunacher Population durch eine chronische Mittelohrentzündung geplagt wurde. Die nach Geschlecht differenzierte Darstellung der Mastoidstruktur findet man in Abbildung 40 . 
Eine chronische Mittelohrentzündung bei bereits abgeschlossener und normaler Pneumatisation kann einen pathologischen Umbau des Mastoids bewirken. Der spongiosaähnliche Umbau der Cellulae mastoideae und die damit verbundene spongiöse Verdichtung spricht also für das vorausgegangene Vorliegen einer Mittelohrentzündung.

Bei der Gegenüberstellung Mastoid und Mittelohr ist eine relative Häufung von Personen mit gesundem Mittelohr und gesundem Mastoid oder eben krankes Mittelohr und krankes Mastoid zu erwarten. Dazu bilden jeweils ein Mittelohr und das zugehörige Mastoid ein Paar. Beide müssen in einem Zustand sein, der eine Befundung der Strukturen erlaubt. In Baunach konnten so 58 Mittelohr-MastoidPaare gebildet werden.

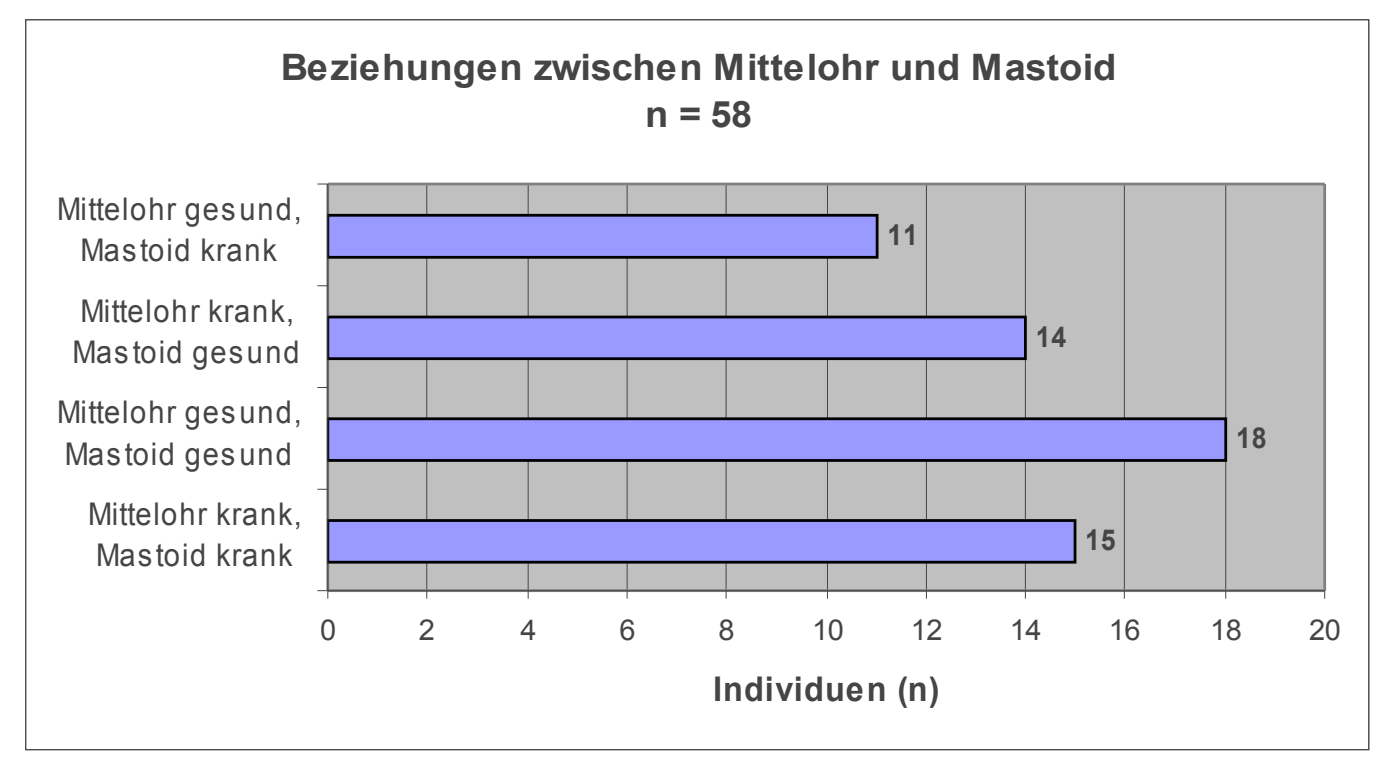

Abbildung 41: Beziehungen zwischen der Erkrankung des Mittelohres und des Mastoids

Als erkrankt galt ein Mastoid, wenn sich in den pneumatischen Räumen Neubildungen, Einschmelzungen oder Gefäßimpressionen an den Septenwänden nachweisen ließen. Auch eine Spongiosierung der Cellulae mastoideae sowie eine primär spongiöse Struktur des Processus mastoideus wurden als krank gewertet. Die Kriterien einer Otitis media sind im Kapitel 3.5.3 erläutert worden. 
Medizinisch erwartet man bei den untersuchten Strukturen Paare mit gleichem Erkrankungs- beziehungsweise Gesundheitsstatus.

So stellt sich dieser Zusammenhang bei der Mehrzahl (33 Paare; 57 Prozent) der untersuchten Fälle dar. Bei diesen 33 Paaren ist sowohl am Mastoid als auch am Mittelohr der gleiche Gesundheitszustand zu erkennen.

Den größten Anteil (31 Prozent) bilden Paare, bei denen sowohl das Mittelohr als auch das zugehörige Mastoid keinerlei pathologische Veränderungen zeigen.

Dagegen weisen 25 Paare, also 43 Prozent der Gesamtmenge, einen vom Mittelohrbefund unabhängigen Gesundheitszustand des Mastoids auf. Die Gruppe der kranken Mittelohren mit gesundem Mastoid (24 Prozent) ist größer als die Gruppe mit isoliert auftretendem kranken Mastoid. Nur elf Paare (19 Prozent) weisen eine isolierte Erkrankung des Mastoids bei gesundem Mittelohr auf.

In der Abbildung 41 findet man die einzelnen Konstellationsmöglichkeiten mit Fallzahlen dargestellt.

Auch bei der Korrelation von erkrankten oder gesunden Nasennebenhöhlen und Mittelohren wird eine pathophysiologisch bedingte Häufung jeweils gleicher Befundqualitäten erwartet.

Das Ergebnis dieser Untersuchung ist in folgendem Diagramm (Abb. 42) dargestellt. Die Abkürzung MO steht für Mittelohr, NNH für Nasennebenhöhlen. 


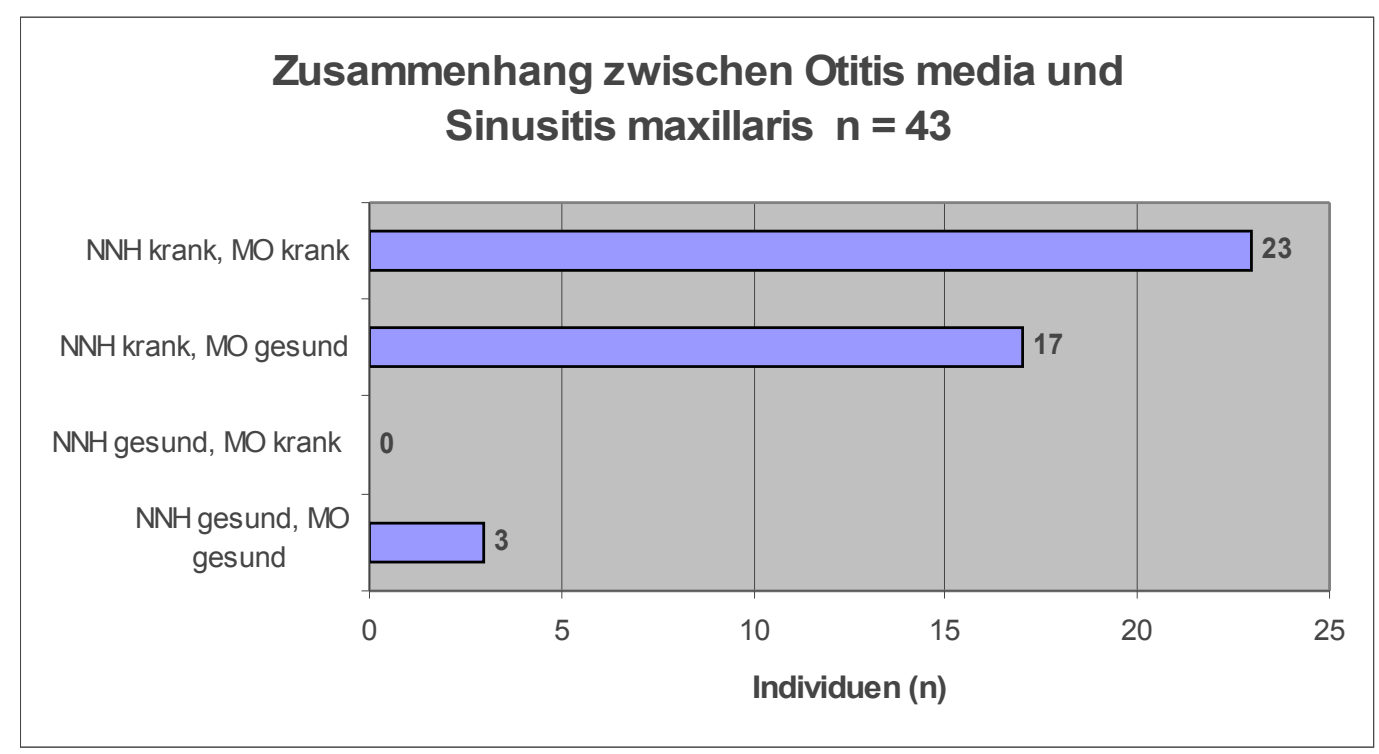

Abbildung 42: Zusammenhang zwischen Mittelohrentzündung und Kieferhöhlenentzündung

Es sind 43 Paare von befundbarem Mittelohr und korrespondierendem Sinus maxillaris auszumachen. Bei weiteren 24 Mittelohren kann keine Untersuchung der Nasennebenhöhlen auf Grund der knöchernen Intaktheit des Gesichtsschädels ohne dessen Zerstörung durchgeführt werden. Diese Mittelohren werden somit nicht in die Untersuchung einbezogen.

Von diesen 43 Paaren zeigt die überwiegende Mehrzahl (93 Prozent) eine pathologische Veränderung im Bereich der Nasennebenhöhlen. Nur in dieser Gruppe sind auch erkrankte Mittelohren zu finden. Von den 40 Paaren mit Sinusitis maxillaris weisen 23 (57,5 Prozent) auch eine Otitis media auf. In 42,5 Prozent der Fälle, also den restlichen 17 Paaren mit erkrankten Nasennebenhöhlen, ist das Mittelohr gesund. Dagegen sind nur 7 Prozent, also drei Paare, im Bereich der Kieferhöhlen gesund. Bei diesen findet sich kein erkranktes Mittelohr. Somit bestätigen sich die in Kapitel 3.5.2 genannten Bedingungen für eine Mittelohrentzündung auch bei der paläopathologischen Untersuchung. Dass Vorhandensein einer Sinusitis stellt also in Baunach eine wesentliche Voraussetzung für eine Otitis dar. 


\subsection{Pathologische Befunde im Schädelinnenraum}

\subsubsection{Anatomische und physiologische Grundlagen}

Bei den umfassenden Aufgaben des Gehirns ist dieses das wohl schutzbedürftigste Organ des Körpers. Um dieser Aufgabe gerecht zu werden, verfügt der das Hirn umschließende Abschnitt des Schädels über mehrere Schutzmechanismen.

Diese werden unterteilt in drei Abschnitte (LIPPERT 1996):

1. verformbare Zone: Kopfschwarte

Als Kopfschwarte bezeichnet man die aus stabilem Bindegewebe aufgebaute Aponeurose (Sehnenplatte) des M. occipitofrontalis. In älteren anatomischen Quellen wird dieser häufig auch als „M. epicranius“ (BERGMANN et al. 1921) bezeichnet. Diese ist fest mit der behaarten Kopfhaut verwachsen und locker mittels subaponeurotischem Bindegewebe mit dem Periost der Schädelknochen verbunden. Das den Kopf schützende Weichgewebe ist somit mehrere Millimeter dick.

2. stabile Zone: Schädelknochen

Ähnlich wie bei den Röhrenknochen zeichnet sich der Aufbau des knöchernen Schädeldaches durch eine sogenannte „Sandwich-Konstruktion“ aus (zwei stabile Schichten sind durch einen locker gebauten Abstandshalter, die Diploë, verbunden).

\section{Verzögerungszone: Liquorräume}

Das zentrale Nervensystem wird allseits vom Liquor cerebrospinalis (Hirnwasser) umspült. Somit muss das Gehirn bei einem Aufprall erst den Liquor verdrängen, bevor es an der Hirnhaut aufschlagen kann. Dadurch kommt es nur selten zu einem direkten Anprall. Unterstützend wird der Liquorraum durch das straffe Bindegewebe der Dura mater (harte Hirnhaut) in einzelne Kompartimente unvollständig untergliedert, so dass sich einwirkende Kräfte nicht ungebremst über den gesamten Liquorraum verteilen können. 
Die Abbildung 43 zeigt eine anatomische Darstellung zur Verdeutlichung der 3 Kompartimente.

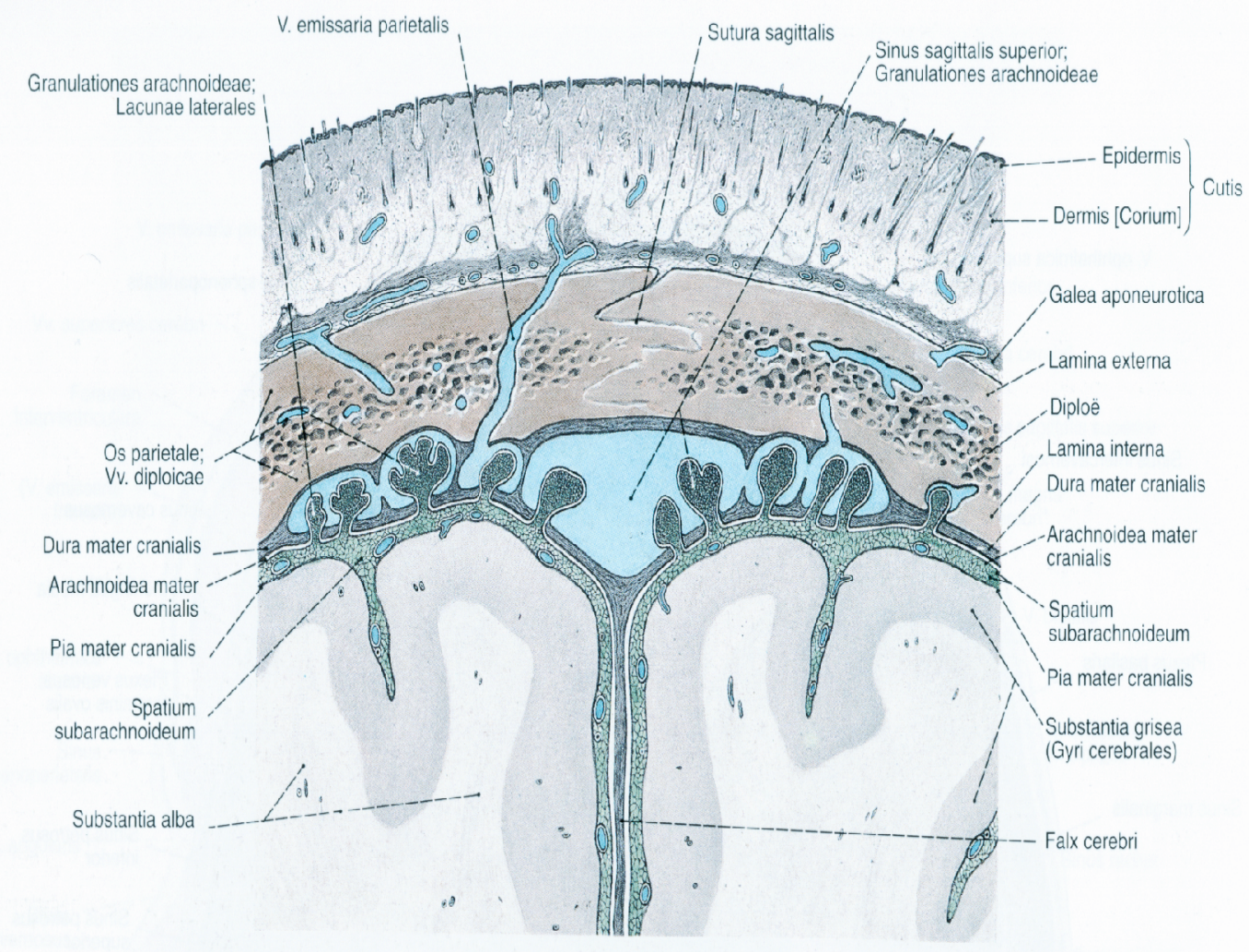

Abbildung 43: Schematisierter Frontalschnitt durch Schädeldach und Hirnhäute am Ansatz der Falx cerebri (Quelle: Sobotta 1993, S. 256)

Somit ist das Gehirn im Schädelinneren optimal vor direkter Gewalteinwirkung geschützt.

Der endocranielle Raum wird durch mehrere Schädelknochen begrenzt. Diese sind nicht durch Synarthrosen im Sinne von synoviale Gelenken sondern durch Knochennähte (Syndesmosen-Synostosen) miteinander verbunden. Im Laufe des Lebens verknöchern diese vollständig in bestimmten Altersgrenzen zu festen Verbindungen und sind somit wichtige Marker zur Altersbestimmung am Schädel. 


\subsubsection{Erkrankungen des Schädelinnenraumes}

In der vorliegenden Population weist eine große Zahl der Skelete Läsionen im Endocranium auf, welche auf meningeale Reizungen schließen lassen.

Die Lamina interna als eine knöcherne Begrenzung des endocraniellen Raumes besitzt im Normalfall eine glatte Oberfläche und bis auf die Impressionen der Meningealarterien keine Abdrücke von Gefäßen. Bedeckt wird die Lamina interna von der Dura mater, welche sich in ein äußeres periostales Blatt und ein inneres meningeales Blatt gliedern lässt. In der Regel stellt sich diese Struktur bei Erwachsenen als morphologische Einheit dar. Die harte Hirnhaut (Dura mater) besitzt eine dem Periost ähnliche Struktur. Bei in diesem Bereich stattfindenden Erkrankungen kann es so zu knöchernen Relikten von Entzündungen oder Blutungen im Schädelinnenraum kommen. Da bei Kindern der Schädel und mit ihm auch die Hirnhäute physiologischen Wachstumsprozessen unterliegen, sind knöcherne Manifestationen im Bereich der harten Hirnhaut in diesem Alter besonders rasch ausgeprägt (SCHULTZ 1993).

In der Population Baunach sind oft Spuren einer meningealen Reizung zu erkennen. Häufig befinden diese sich an mehreren Stellen im Schädel. Verschiedene Erkrankungen zeigen allerdings ein charakteristisches Verteilungsmuster von Entzündungsherden und somit speziellen Verteilungsmustern bestimmter Hirnhautreizungen.

Beispielsweise findet man bei einer tuberkulösen Meningitis sehr häufig stecknadelkopfgroße Knötchen (Druckatrophie durch Tuberkelgranulome) überwiegend basal, also an den tieferen Stellen im Schädel wie zum Beispiel der mittleren und hinteren Schädelgrube (HUEBSCHMANN 1928). Dies erklärt auch die klinischen Symptome wie Hirnnervenausfälle und den durch Verklebung der Aperturae laterales et mediana (Foramina Luschkae und Magendi) verursachten Hirndruckanstieg durch Hydrocephalus internus (BODECHTEL 1963).

Da eine Vielzahl der Individuen die für eine tuberkulöse Meningitis typischen Krankheitszeichen und Verteilungsmuster zeigt, widmet sich der folgende Abschnitt dieser wichtigen und schwerwiegenden chronischen Infektionskrankheit. 


\subsubsection{Tuberkulöse Meningitis}

Die Tuberkulose ist eine Infektionskrankheit, welche als Epidemie auftritt und dann allmählich einen endemieartigen Charakter annimmt. Durch ihre Neigung zur chronischen Infektion und ihre leichte Verbreitung kann sie als Volkskrankheit bezeichnet werden. Damit wird deutlich, dass eine Tuberkulose nicht nur eine Angelegenheit des erkrankten Individuums ist. Durch ihre chronischen Organschädigungen verbunden mit hohem Infektionsrisiko besitzt diese Erkrankung eine große soziale Bedeutung.

Prinzipiell kann diese Infektionskrankheit durch hämatogene Streuung nach Erstinfektion den gesamten Körper treffen.

Durch den chronischen Erkrankungscharakter können Spuren einer Tuberkulose sich auch knöchern manifestieren. Die Methoden der Paläopathologie können somit eine Infektion mit Mycobacterium nachweisen (SCHULTZ 1999).

Eine häufige Manifestationsform einer hämatogen ausgebreiteten Tuberkulose ist die Leptomeningitis tuberculosa. Bei der paläopathologischen Untersuchung der Schädel aus Baunach sind häufig Spuren im Sinne einer chronischen oder akut exacerbierten Leptomeningitis tuberculosa zu finden.

Die tuberkulöse Meningitis ist eine durch das Mykobacterium tuberculosis verursachte endocranielle Infektion im Bereich der Hirnhäute.

Diese ist fast nie als Primärinfektion zu sehen. Voraussetzung ist eine Infektion mit dem äußerst umweltstabilen Bakterium auf aerogenem Weg (BRAUN, RENZ-POLSTER 1999). Diese Stabilität des Erregers beruht auf seiner wachsartigen Hülle, welche für die Immunabwehr eine schwer lysierbare Barriere darstellt (MIKSITS, HAHN 1999). Nach einigen Autoren kann auch die orale Aufnahme von Mycobacterium tuberculosis bzw. dem weniger humanpathogenen Mycobacterium bovis zur Erkrankung führen (ASCHOFF 1919; VINCENT, GUTIERREZ PEREZ 1999). Mycobacterium bovis ist ein in Rindern und anderen Haustieren vorkommendes Mycobacterium. Die Tiere können durch eine Eutertuberkulose infizierte Milch ausscheiden. Untersuchungen aus einer Zeit mit epidemisch vorkommender TBC vermuten über zehn Prozent Haustier-assoziierter Tuberkuloseerkrankungen der untersuchten Bakterienstämme (LINDIG 1964). 
Untersuchungen zu Beginn des 20. Jahrhunderts beschäftigen sich ausgiebig mit der TBC-Übertragung zwischen Mensch und Nutzvieh. Häufig werden bovine Mycobakterien im menschlichen Sektionsgut nachgewiesen, wobei hier vor allem Kinder ein erhöhtes Ansteckungsrisiko besitzen (TAUTE 1907). Die Erkrankung bildet dann ihren Primärkomplex in Form der Hals-Lymphknoten-Tuberkulose, der Kehlkopf-Tuberkulose, der Magen-Darm-Tuberkulose oder, bei Milchkontakt mit der Haut, eine cutane Tuberkulose aus.

Die Abwehrlage und die aufgenommene Bakterienmenge entscheiden über den Krankheitsverlauf des Organismus. So führt eine Infektion mit Mycobacterium tuberculosis nicht zwingend zur manifesten Erkrankung. Nur etwa 5 bis 10 Prozent der Infizierten erkranken an der Tuberkulose (DEUTSCHES ÄRZTEBLATT, Jg.105, Heft 7). Ein immunkompetentes Individuum kapselt die aufgenommenen Tuberkel ab und inaktiviert diese somit. Es bilden sich granulomatöse Herde, welche nach längerem Bestehen über das Zwischenstadium der sogenannten Verkäsung (käsige Nekrose), auch mineralisieren können. Die Krankheit bricht nicht aus.

Die Entstehung von Granulomen ist als ein Eingrenzugsversuch des zellulären Anteils der Immunantwort zu deuten (RASSNER 2000). Bei schwacher Abwehrlage und insbesondere bei Mangelernährung (KOLLATH 1947) können die Bakterien allerdings das umgebende Gewebe einschmelzen und dabei Anschluss an die Gefäßversorgung erlangen. Darüber ist eine hämatogene Aussaat der Tuberkelbakterien in den gesamten Körper möglich. Die Erkrankung begünstigende, resistenzmindernde Faktoren wurden bei den Nasen- und Nasennebenhöhlenerkrankungen aufgeführt.

Gelangt Mycobacterium tuberculosis auf hämatogenen Weg an die Meningen, kann es zu einer tuberkulösen Meningitis mit den typischen Veränderungen an den Knochen der Schädelbasis kommen.

Im Bildanhang sind verschiedene Erkrankungsausprägungen einer endocraniellen Tuberkulosinfektion aufgeführt. 


\subsubsection{Ergebnisse Schädelinnenraum}

Als an Tuberkulose erkrankt gelten Individuen, an deren Schädelbasis man die durch Druckatrophie der Tuberkelgranulome oder die durch eine osteolytisch wirksame Entzündungseaktion verursachten Grübchen (STOCHDORPH 1990) findet. Diese Tuberkelimpressionen erscheinen als flache Grübchen, die sehr häufig auch miteinander konfluieren. Oft sind auch in unmittelbarer Umgebung der Grübchen Zeichen meningealer Reizungen wie netzartige Gefäßimpressionen, eine fein poröse Knochenoberfläche und/oder kleine Neubildungen zu erkennen. Bei der Leptomeningitis tuberculosa finden sich diese Krankheitsmanifestationen meist an der Schädelbasis in der Fossa cranii anterior, media und posterior (SCHULTZ 1999, 2001, TEMPLIN 1993).

Für diese Untersuchung konnten alle Schädel ausgewertet werden, bei denen mindestens ein an den Schädelgruben beteiligter Knochen für eine Befundung zur Verfügung stand. Ein Individuum gilt als an Tuberkulose erkrankt, wenn sich dort morphologisch an mindestens einer Stelle der makroskopische oder mikroskopische Verdacht auf die Krankheit ergibt.

Wie auch schon zuvor bei anderen Erkrankungen ist die Häufigkeit der tuberkulösen Meningitis bei Männern größer (Abbildung 44). 


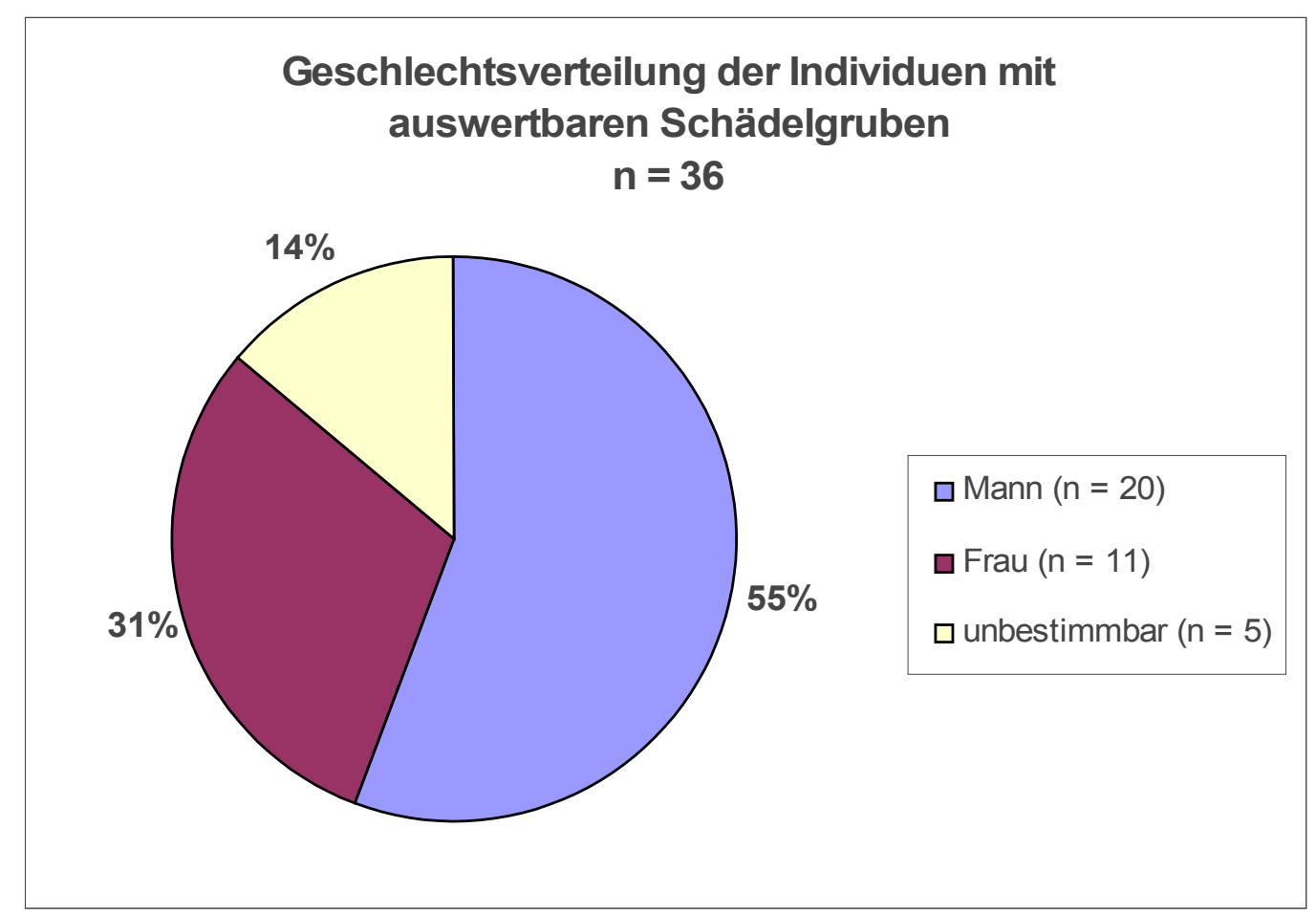

Abbildung 44: Geschlechtsverteilung der Individuen mit Schädelgruben

Bei der geschlechtsbezogenen Verteilung des Verdachtes auf tuberkulöse Meningitis ergibt sich folgendes Diagramm (Abbildung 45):

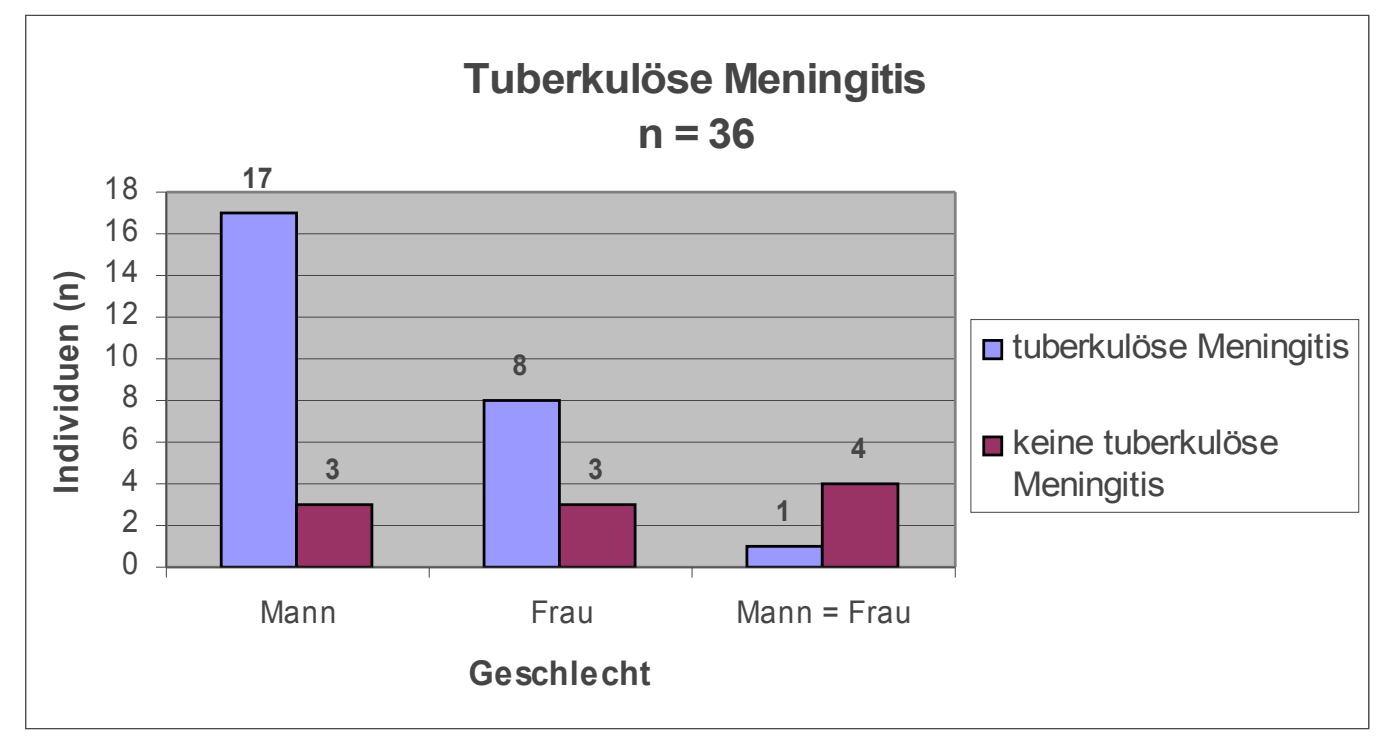

Abbildung 45: Geschlechtsspezifische Verteilung der tuberkulösen Meningitis 
Es fällt auf, dass unbestimmbare Individuen, in Baunach zumeist Kinder, geringere Erkrankungsfälle zeigen als erwachsene Individuen (Abbildung 46).

Mögliche Gründe dafür sind der geringe Altersdurchschnitt dieser Gruppe und die damit verbundene geringere Wahrscheinlichkeit, an dieser chronischen Infektion zu erkranken und somit pathologisch veränderte Schädelknochen aufzuweisen.

Bei den Skeleten mit Spuren einer tuberkulöser Meningitis findet man jedoch fast immer auch die oben aufgeführten Zeichen einer unspezifischen Hirnhautreizung. Eine meningeale Reaktion muss jedoch nicht zwangsläufig durch Tuberkulose hervorgerufen werden. Auch die in den Kapiteln zuvor beschriebenen unspezifischen Infektionen des Schädels können durch Aussaat der Erreger zu einer Reizung der Meningen führen.

In der Population Baunach zeigen nur drei Männer, drei Frauen und vier nicht bestimmbare Individuen keine pathologischen Veränderungen an den Schädelgruben. An der überwiegenden Mehrzahl der Schädel finden sich Spuren akuter oder chronischer meningealer Reizzustände.

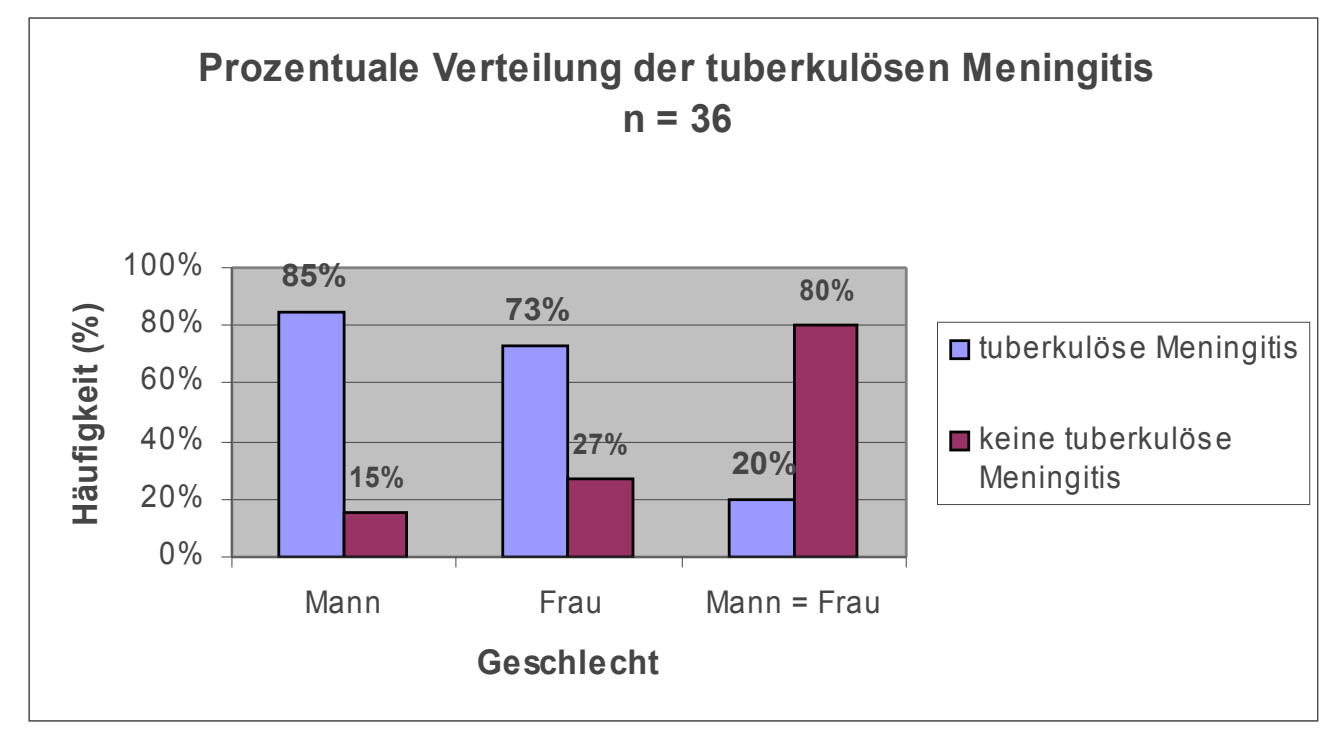

Abbildung 46: Geschlechtsdifferenzierte prozentuale Verteilung der tuberkulösen Meningitis 
Bei der prozentualen Aufteilung ergibt sich für Männer mit 85 Prozent und Frauen mit 73 Prozent eine deutliche Krankheitshäufigkeit für die tuberkulöse Meningitis. Grafisch dargestellt wird dies in der Abbildung 46.

Da diese Krankheit chronisch verläuft und das Individuum längere Zeit klinisch asymptomatisch bleiben kann, ist bei großer anzunehmender "Dunkelziffer" von erkrankten Personen diese Erkrankungshäufigkeit durchaus vorstellbar.

Bei der Untersuchung findet man an den Schädelgruben nicht nur spezifische Hinweise für Tuberkulose. Häufig sind auch unspezifische Zeichen für eine durchgemachte Hirnhautreizung zu beobachten. Diese meningealen Reaktionen kommen oft in der Umgebung von tuberkulosetypischen Läsionen vor. Oft sind diese aber auch die einzigen Repräsentanten einer Erkrankung der Hirnhäute. Die folgende Abbildung 47 stellt die einzelnen Häufungen dar.

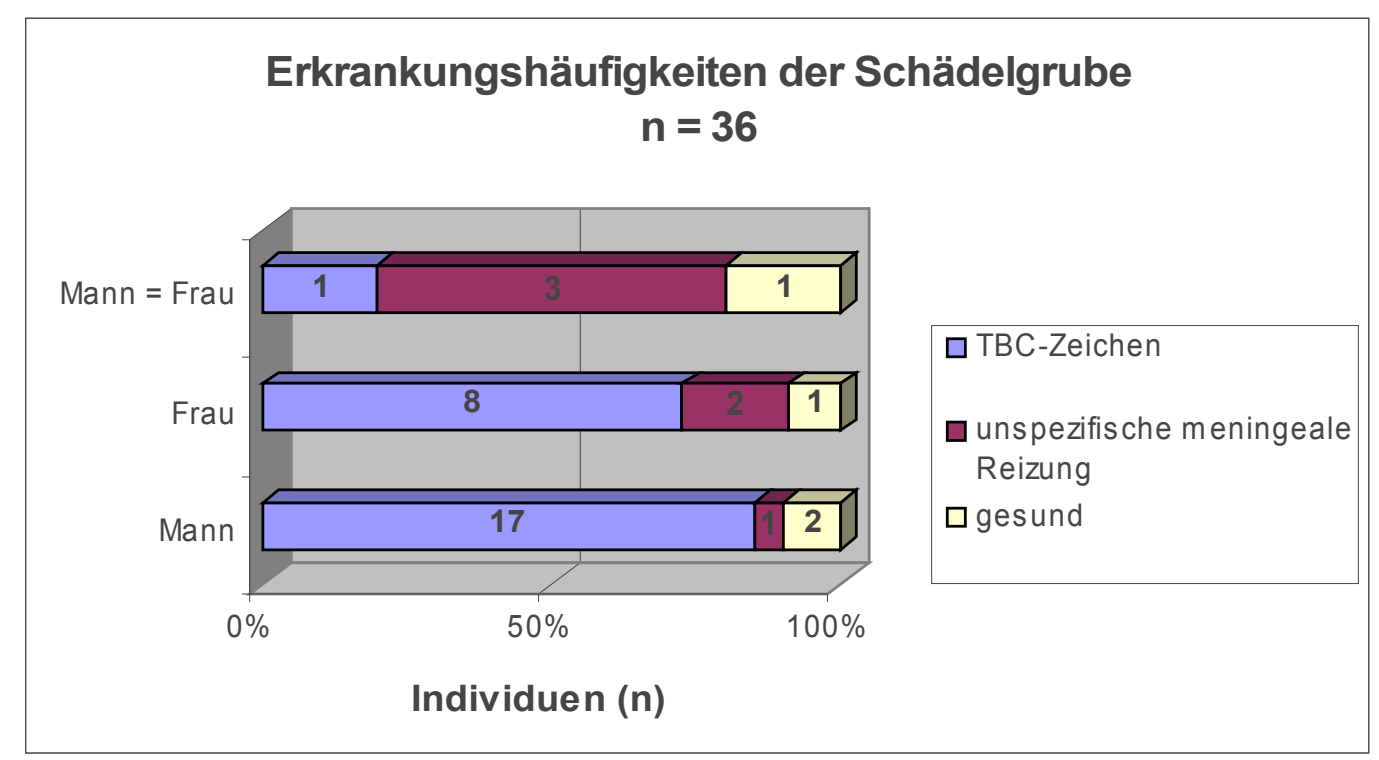

Abbildung 47: Verteilung der Fälle mit Spuren meningealer Reaktionen in den Schädelgruben

Auch unspezifische Zeichen von meningealen Reaktionen sind als krankhafte Prozesse zu werten. Eine gesunde Lamina interna besitzt eine glatte Oberfläche und keine Neubildungen oder poröse Auflagerungen. Diese sind als Zeichen einer ent- 
zündlichen Reaktion der Hirnhäute zu werten und stellen somit Hinweise für Meningitiden anderer Genese dar. Aber auch eine TBC kann solche Läsionen verursachen (SCHULTZ 1987, 1993, 2001, 2003).

Unspezifische meningeale Reaktionen an der Hirnbasis, also an den schon genannten Schädelgruben, stellen ein Zeichen für eine Meningokokken-Meningitis dar (STEINBERG 1933). Diese Infektion wird durch Meningokokken verursacht, deren Eintrittspforte eine Primärinfektion im Sinne eines Meningokokkenkatarrh im Nasen-Rachen-Raum darstellt (SCHUERMANN 1958). Der Häufigkeitsgipfel findet sich im infantilen und juvenilen Lebensabschnitt (ASCHOFF 1919). In Baunach stellen sich vergleichbare Ergebnisse dar. Bei der Gruppe Mann = Frau finden sich diese Zeichen prozentual am häufigsten (60 Prozent). Da hier die meisten Kinder und Jugendlichen zu finden sind kann man annehmen, dass die Infektion mit Meningokokken eine Ursache für die meningealen Reizungen ist.

Aber auch eine Tuberkulose hat bei der kindlichen Infektion einen Erkrankungsgipfel im zweiten bis dritten Lebensjahr. Da eine unbehandelte tuberkulöse Hirnhautentzündung durchschnittlich nach drei Wochen (HERAUSGEBERAUFSATZ 1952) zum Tode führt, sind bei derartig erkrankten Kindern Spuren dieser Erkrankung am Knochen erwarten. Der kindliche Knochen zeigt sehr schnell Manifestationen einer meningealen Reizung (SCHULTZ 1993, 1999, 2001).

Fasst man die Gruppen der an Hirnhauttuberkulose und an einer unspezifischen Hirnhautentzündung erkrankten Personen zu einer Gruppe zusammen, ergeben sich folgende Häufigkeiten (Abbildung 48). 


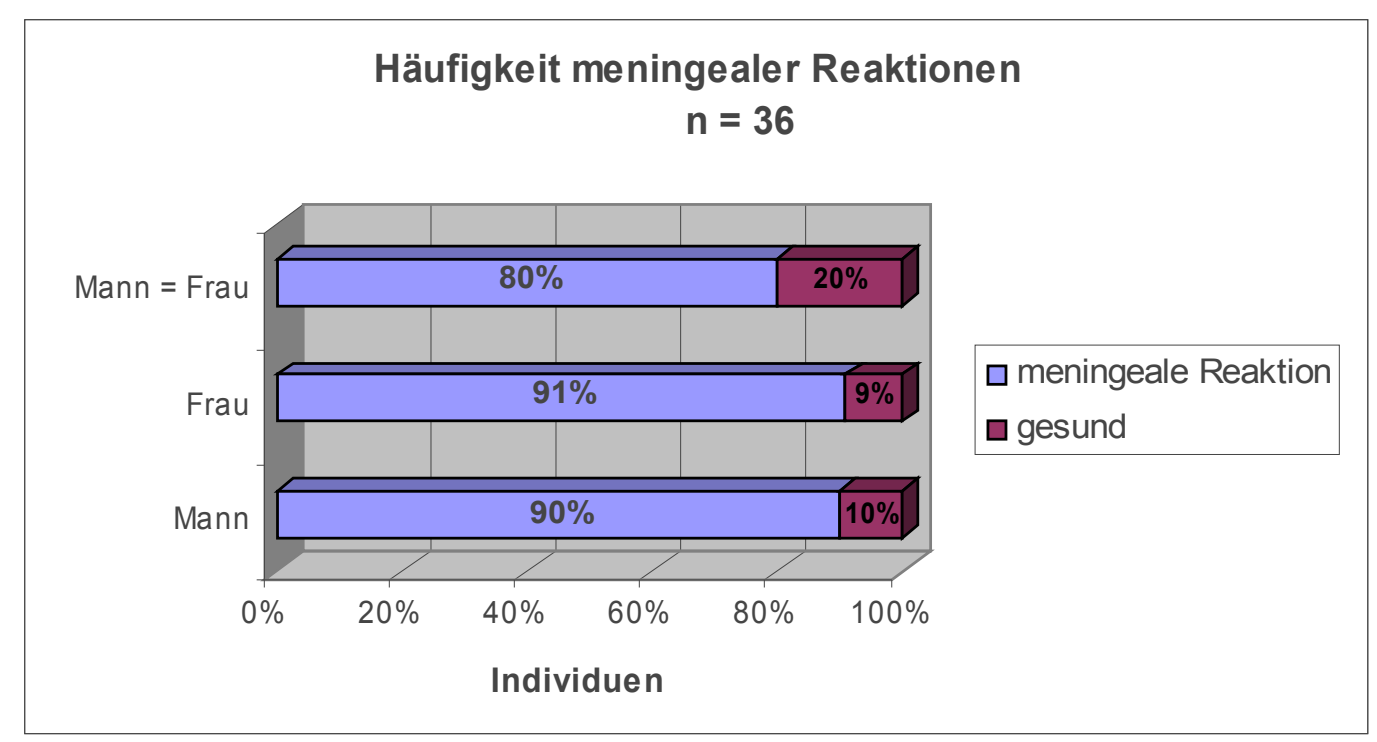

Abbildung 48: Prozentuale Verteilung der meningealen Reaktionen

Spuren einer meningealen Reizung lassen sich bei 80-90 Prozent der Baunacher Skelete feststellen. Ähnliche Erkrankungshäufungen aus vergleichbaren Epochen sind dabei auch in anderen Populationen in Mittel- und Süddeutschland zu finden (ROUMELIS 2007; GRESKY et al. 2003).

Männer und Frauen zeigen fast identische Erkrankungshäufigkeiten. Die Häufigkeit meningealer Reaktionen bei der Gruppe Mann = Frau ist mit 80 Prozent etwas niedriger als bei Männern und Frauen. 


\subsection{Pathologische Befunde am Schädeldach}

\subsubsection{Anatomische Grundlagen}

Das Schädeldach wird beim Menschen aus dem Os frontale, den Ossa parietalia und dem Os occipitale gebildet, die im Laufe des Lebens über Knochennähte zusammenwachsen.

Es werden am Schädeldach drei Schichten unterschieden. Die äußerste Schicht ist die Lamina externa. Diese geht fließend in den Teil des Knochens über, der als Diploë bezeichnet wird. Nach innen abschließend und somit endocraniell gelegen folgt die Lamina interna.

Bei Erkrankungen des Schädeldaches findet man makroskopisch von außen sichtbar die erkrankten Läsionen zunächst auf der Lamina externa. Bei einer weiterführenden mikroskopischen Befundung kann auch die Beschaffenheit der Diploë und der Lamina interna beurteilt und so häufig der Infektionsweg geklärt werden. In der Diploë ist der Knochen nicht massiv wie in den beiden anderen Abschnitten, sondern als Schwammknochen spongiös aufgebaut. Die dort befindlichen Venen stehen mit den Venen der Kopfhaut und den Blutleitern des Gehirns in Verbindung. So können diese Gefäße in begrenztem Umfang als Kollateralkreislauf bei Sinusvenenthrombose dienen. Aber durch diese Verbindung können auch Infektionen hämatogen von der äußeren Kopfhaut zu den Meningen und darüber bis zum Gehirn vordringen.

\subsubsection{Erkrankungen des Schädeldachs}

Eine Infektion der Schädelaußenfläche kann durch direkten Befall der Kopfhaut mit Krankheitserregern verursacht werden, wie es zum Beispiel bei Furunkeln oder einem Erysipel der Fall ist. Eine chronische Infektion mit Kopfläusen oder Pilzen wirkt durch die geschädigte Hautbarriere ebenfalls begünstigend für einen bakteriellen Befall des Schädeldaches und der darüber liegenden Galea aponeuro- 
tica bzw. Kopfschwarte (ZIEGLER 1898, RASSNER 2000). Auch bei traumatischen Läsionen mit Verletzung der Kopfschwarte können Infektionserreger eindringen und Spuren an der Lamina externa des Schädeldaches hinterlassen.

Die Knochen des Schädeldaches können aber auch durch ein Trauma geschädigt werden, bei dem je nach Verletzungsart eine Fraktur, ein subperiostales Hämatom oder auch eine Osteomyelitis entstehen kann. Das Schädeldach ist an archäologischen Funden durch seine große Fläche und die exponierte Lage sehr gut zu untersuchen. Allerdings birgt dies auch eine größere Zerstörungsgefahr im Sinne der Diagenese, z. Bsp. Erde und Sand, Wasser, Wurzeln, Pilze, Algen, Bakterien, Arthropoden (SCHULTZ 1997).

Die freie Schädeldecke ist durch die Muskelmarken der mimischen Muskulatur sowie durch die Kau- und Nackenmuskulatur begrenzt. Das von diesen Strukturen umschlossene Areal des Schädeldaches wird von der Galea aponeurotica bedeckt. Erkrankungen, die zu einer Schädigung in diesem Bereich führen können, wurden bereits genannt.

In den Bildern 17 und 18 im Anhang werden makroskopische und elektronenmikroskopische Eindrücke einer ausgeprägten Läsion des Schädeldaches eines Baunacher Individuums dargestellt.

Die gesunde Lamina externa ist glatt und zeigt nur vereinzelte kleine Foramina, durch die die Emissarvenen eine Verbindung zwischen Kopfhaut und Diploë herstellen. Bei Erkrankungen des Schädels wird die Oberfläche zunehmend poröser, was auf eine Zunahme der Blutflussmenge, also eine Hypervaskularisation, schließen lässt. Auch können sich fein-poröse Platten als Zeichen der Organisation einer ehemals hämorhagischen Läsion bilden und schließlich zu einer Verdickung integriert werden. Bei entzündlich bedingten Läsionen werden auch osteolytische Grübchen und kleine zapfenartige Neubildungen beobachtet. 


\subsubsection{Ergebnisse Schädeldach}

Alle Skelete, bei denen eine Beurteilung der Lamina externa des Schädeldaches möglich ist, werden für die folgende Auswertung einbezogen. Dazu zählen auch die Individuen, bei denen nur Fragmente des Schädeldaches erhalten sind.

In der Population Baunach können so 38 Individuen berücksichtigt werden.

Das folgende Diagramm der Abbildung 49 zeigt die Verteilung der vorhandenen Schädeldächer nach dem Geschlecht.

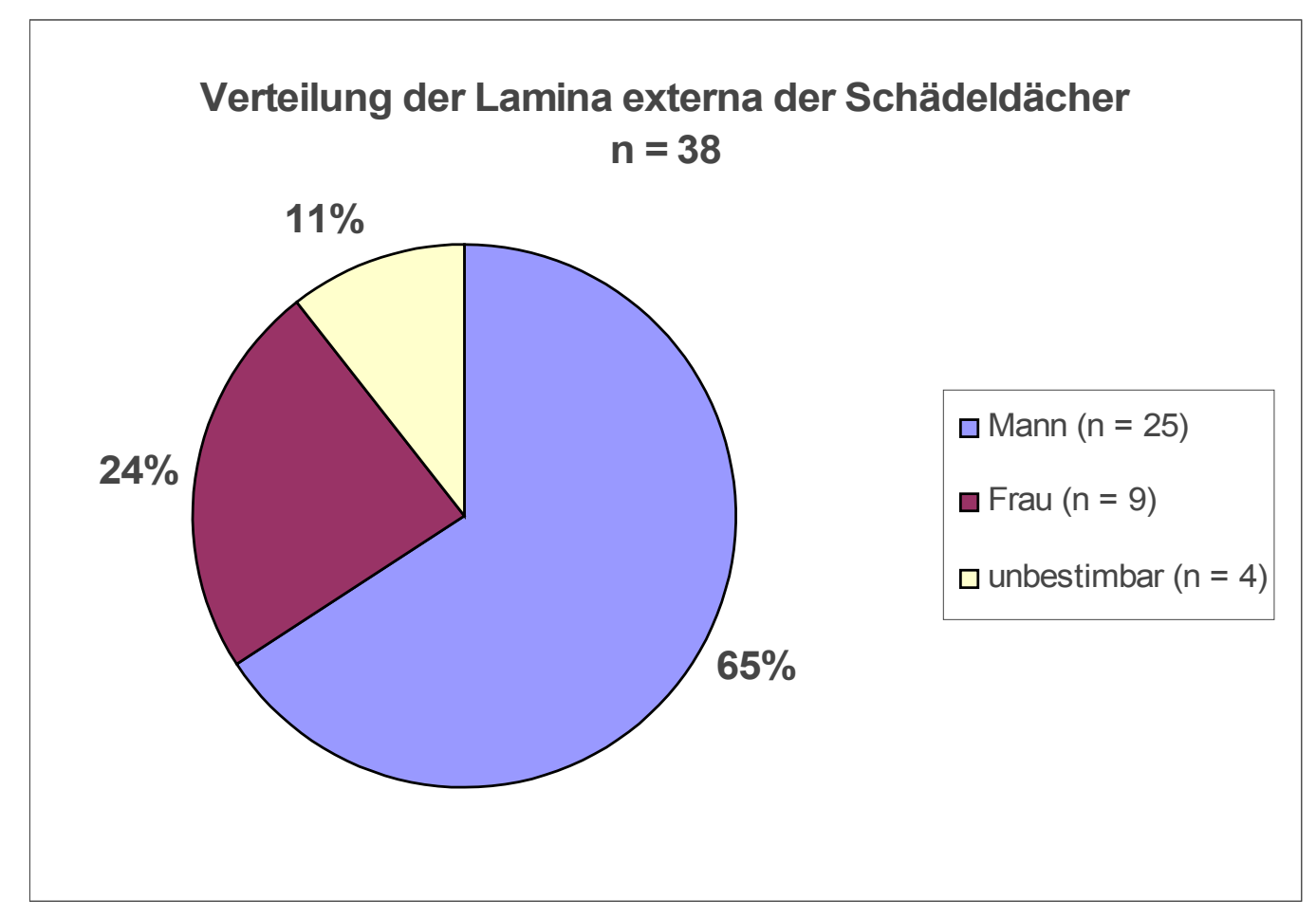

Abbildung 49: Geschlechtsspezifische Verteilung der Individuen mit Schädeldach

Bei der Unterscheidung in kranke und gesunde Lamina externa des Schädeldachs zeigt sich, dass das männliche Geschlecht nicht nur in der absoluten Anzahl der verwertbaren Schädeldächer, sondern auch in der prozentualen Häufigkeit einer pathologisch veränderten Lamina externa deutlich überwiegt. Dies wird in der Abbildung 50 dargestellt. 


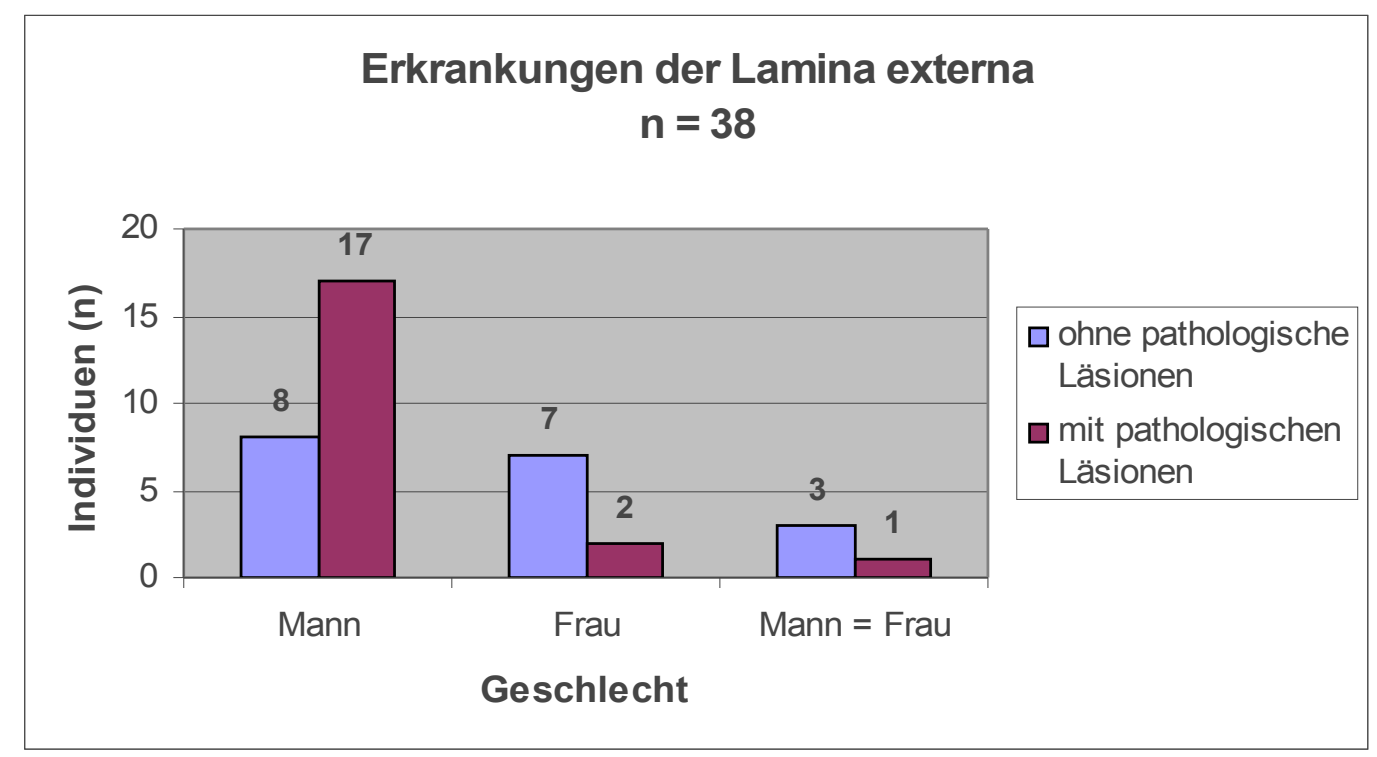

Abbildung 50: Geschlechtsspezifische Verteilung Kopfschwartenentzündung Lamina externa

Es finden sich von 25 männlichen Individuen 17 Schädeldächer mit pathologischen Veränderungen (68 Prozent).Dagegen sind nur 22 Prozent der weiblichen Schädeldächer erkrankt. Auch bei den Individuen, deren Geschlecht nicht bestimmt werden kann, sind nur 25 Prozent mit pathologischen Läsionen vertreten. Männer aus der Baunacher Population sind also weitaus stärker als Frauen oder Kinder an Läsionen des Schädeldaches erkrankt.

Als pathologische Läsion zählt eine poröse Oberfläche innerhalb der Grenzen der Galea aponeurotica, was als generalisierte Kopfschwartenentzündung zu interpretieren ist. Die Entzündung kann sich durch die umgebenden Muskelmarkenansätze und die daraus resultierende stabile räumliche Begrenzung nur schwer außerhalb des Schädeldaches ausbreiten.

Auch eine einzelne Läsion auf der physiologisch glatten Schädeldachcorticalis ist als pathologisch verändert zu werten. Bei hämorrhagischer Komponente, also plattenartigen oder porös verdickten Auflagerungen mit vermehrten Gefäßimpressionen in der Peripherie auf sonst gesunder Lamina externa, ist ein Trauma eine mögliche Ursache. Bei einem Schlag auf den Kopf kann es zu einer subperiostalen 
Einblutung mit einer umgebenden ödematösen Gewebeschwellung kommen, die langsam wieder organisiert und integriert wird. Die Folge ist eine einzelne, von der Umgebung scharf abgegrenzte Auflagerung.

Bei sehr starker Gewalt oder der Verwendung eines spitzen Gegenstands kann eine offene Wunde entstehen über die Keime eintreten, die dann wiederum eine Kopfschwartenentzündung verursachen. Durch entsprechenden Kopfschutz (z.B. Lederhauben oder Helme) kann die einwirkende scharfe Gewalt zu einem einfachen Schlag gemindert werden. Die Kombination aus Kopfschwartenentzündung und hämorrhagischer Läsion kann also für ein mit starker Kraft oder Waffengewalt ausgeführtes stumpfes Trauma sprechen. Dieser Verletzungsvorgang ist für kriegerische Auseinandersetzungen typisch. Aber auch ein starker Schlag oder Sturz auf den Schädel im Sinne eines Bagatelltraumas kann eine hämorrhogische Läsion auf dem Schädeldach verursachen.

Von chirurgisch bedingten Infektionen durch Operationen am Schädeldach ist bei dieser Population nicht auszugehen. Im Gegensatz zur Steinzeit, aus der Schädel mit erfolgreichen Trepanationen erhalten sind, findet man aus der Zeit der Karolinger keine derartigen Funde in Mitteleuropa (RÜSTER 1984).

Bei der Verteilung dieser durch mögliche kriegerische Handlungen begünstigten Veränderungen des Schädeldaches zeigt sich in Abbildung 51, dass das männliche Geschlecht hier eindeutig überwiegt.

Sicher traumatisch bedingte Läsionen lassen sich nur bei erwachsenen männlichen Schädeln finden. 


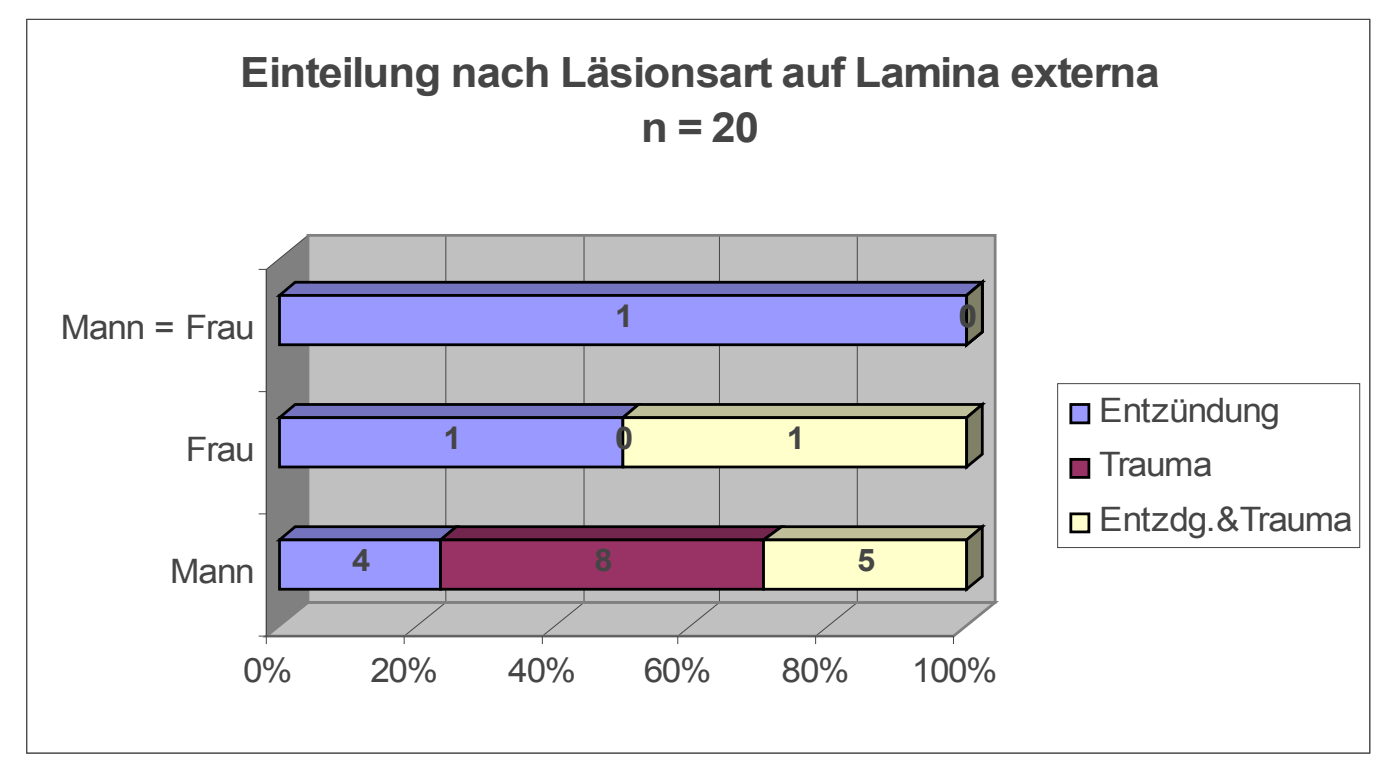

Abbildung 51: Läsionen im Bereich der Lamina externa differenziert nach

Geschlecht

Auch dieses spricht für einen eher traumatisch bedingten Ursprung der Schädeldachveränderungen der Männer, da heute bei einer durch Kopfparasiten wie Pediculus humanus capitis verursachten Kopfschwartenentzündung das weibliche Geschlecht sowie die Kinder deutlich überwiegen (DERMATOLOGIE IN PRAXIS UND KLINIK 1980).

Eine alleinige Kopfschwartenentzündung ohne Trauma findet man bei 4 von 25 Männern (16 Prozent). Dieser Wert liegt deutlich unter denen der Frauen und Kinder. Somit ist es wahrscheinlich, dass eine Pediculosis capitis eine wichtige Ursache für eine Kopfschwartenentzündung nicht männlicher Individuen in Baunach darstellt. 


\subsection{Pathologische Befunde in den Sulci der venösen Hirnblutleiter}

\subsubsection{Anatomische Grundlagen}

Der venöse Rückstrom im Gehirn unterscheidet sich von dem des restlichen Körpers. Das gesammelte venöse Blut des Gehirns fließt nicht durch Venen, sondern durch Duplikaturen der harten Hirnhaut. Diese Blutleiter, die Sinus durae matris, besitzen im Gegensatz zu den Venen keine Muskelwandung oder Venenklappen, die einen Rückfluss des Blutes verhindern. Durch den Einbau des venösen Blutgefäßes in die straffe und feste Dura mater kann sich der Sinus nicht verengen oder erweitern. Das Blut wird nur durch die arterielle Pulsation und nicht durch Muskelpumpe und Venenklappen unterstützt zurück zum Herzen gepumpt.

Bei Erkrankungen der Blutgefäße in der harten Hirnhaut ist der Blutabfluss des Gehirns gefährdet beziehungsweise eingeschränkt. Dies kann zu einer Stauung des Blutes im Gehirn mit den möglichen klinischen Symptomen führen, die für das jeweilige betroffene Gefäß typisch sind. So findet man bei einer Thrombose des Sinus sagittalis superior, welcher mit Venen der Nasenhöhle in Verbindung steht, häufig Nasenbluten oder eine Cyanose des Gesichts. Dagegen verrät sich eine Thrombose der Sinus transversus durch eine retroaurikuläre Schwellung und Ödeme, da dieser Sinus mit einer Vene des Processus mastoideus kommuniziert. Bei einer Thrombose des Sinus cavernosus, welcher Blut aus der Orbita drainiert, wird ein Lidödem beobachtet. Auch ist in diesem Fall der Bulbus protrahiert und die Venen des Augenhintergrundes geschwollen, was klinisch mit einer Sehminderung einhergeht. Dieser in Zeiten ohne adäquate medizinische Versorgung nicht selten auftretende Pathomechanismus wurde früh beschrieben und häufig im Wochenbett beobachtet (JÜRGENSEN 1889). 


\subsubsection{Erkrankungen der venösen Hirnblutleiter}

Die Ursachen einer Sinusthrombose decken sich in vieler Hinsicht mit den pathogenetischen Faktorenen der normalen venösen Stase.

Zusätzlich zu diesen Faktoren tritt eine Thrombose der Sinus häufig bei marantischen oder kachektischen Personen auf. Kinder sind in diesem Fall häufig betroffen. Auch bei Schwangerschaften und im Wochenbett, wobei ein insgesamt erhöhtes Thromboserisiko besteht, tritt diese Erkrankung relativ häufig auf (QUANDT 1969). Einen Überblick über mögliche Ursachen einer Sinusvenenthrombose (SVT) gibt Tabelle 4 (BRANDT et al. 2003). 


\begin{tabular}{|c|c|}
\hline Ursachen einer SVT & Mögliche Beispiele \\
\hline Hormoneller Zusammenhang & $\begin{array}{ll}\text { - } & \text { Gravidität } \\
\text { - } & \text { Wochenbett } \\
\text { - } & \text { postabortiv }\end{array}$ \\
\hline Lokale Kompressionen & $\begin{array}{ll}\text { - } & \text { Meningeome } \\
\text { - } & \text { Obere Einflussstauung }\end{array}$ \\
\hline $\begin{array}{c}\text { Autoimmunerkrankungen / } \\
\text { Vaskulitiden }\end{array}$ & $\begin{array}{ll}\text { - } & \text { Lupus erythematodes } \\
\text { - } & \text { Morbus Crohn } \\
\text { - } & \text { Colitis ulcerosa }\end{array}$ \\
\hline Trauma & $\begin{array}{ll}\text { - } & \text { Schädel-Hirn-Trauma } \\
\text { - } & \text { Schädelfraktur }\end{array}$ \\
\hline Gerinnungsstörungen & $\begin{array}{ll}\text { - } & \text { Protein-C-Mangel } \\
\text { - } & \text { Protein-S-Mangel } \\
\text { - } & \text { Antithrombin-III-Mangel }\end{array}$ \\
\hline Neoplasien & $\begin{array}{ll}\text { - } & \text { Polycythaemia vera } \\
\text { - } & \text { Lymphatische und } \\
& \text { Myeloische Leukämien }\end{array}$ \\
\hline Infektionen & $\begin{array}{l}\text { - Systemische bakterielle, } \\
\text { virale, parasitäre und } \\
\text { mykotische Infektionen } \\
\text { - } \quad \text { lokale Infektionen am Schädel }\end{array}$ \\
\hline Andere Allgemeinerkrankungen & $\begin{array}{ll}\text { - } & \text { schwere Dehydratation } \\
\text { - } & \text { Marasmus } \\
\text { - } & \text { Leberzirrhose } \\
\text { - } & \text { Nephrotisches Syndrom }\end{array}$ \\
\hline
\end{tabular}

Tabelle 4: Ursachen und klinische Beispiele einer Sinusvenenthrombose (SVT)

Ist der den Sinus verschließende Thrombus zusätzlich durch Bakterien infiziert, kann sich eine infizierte Sinusthrombose entwickeln. Es entsteht eine eitrige Entzündung in der Umgebung der Thrombose, welche direkt per continuitatem oder hämatogen weitere entzündliche Komplikationen auslösen kann. Die septische Meningoencephalitis ist eine lebensbedrohliche Komplikation. Hervorgerufen 
wird diese durch Entzündungen, welche in der Nachbarschaft der Sinus ablaufen und Anschluss an die Sinusstrukturen finden. Ein Beispiel für eine solche Entzündung ist eine eitrige Entzündung des Felsenbeines, welche sich im Anschluss an eine Otitis media ausbilden kann. Auch eine bakterielle Sinusitis frontalis kann zu einer solchen lebensbedrohlichen Komplikation führen. Bedingt durch den venösen Abfluss von Blut aus dem Gesichtsbereich über die Hirnsinus können auch Furunkel oder Erysipele des Gesichts eine Thrombose der venösen Blutgefäße des Gehirns auslösen. Entzündungen der Kopfhaut können sich über die Venae emissariae in den endocraniellen Raum ausbreiten und dort eine Sinusthrombose verursachen. Entzündliche und septische Metastasen streuende Herde werden ebenfalls auch oft in den Lungen, den Gelenken oder den Herzklappen gefunden (BODECHTEL 1963).

Eine Behandlung einer solchen infizierten Sinusthrombose war bis zur Einführung der Antibiotika nicht möglich und führte fast immer zum Tod des Individuums.

\subsubsection{Ergebnisse Sinus sagittalis superior}

Es können insgesamt die Sinus sagittales superiores von 37 Baunacher Skeleten untersucht werden. Notwendig dafür sind eine representative Erhaltung sowie die komplette Einsehbarkeit des Sulcus des Sinus sagittalis superior, was für eine verlässliche Befundung unerlässlich ist. Die Aufteilung der für die Statistik aussagekräftigen Sinus sagittalis superior zeigt folgende Abbildung 52. 


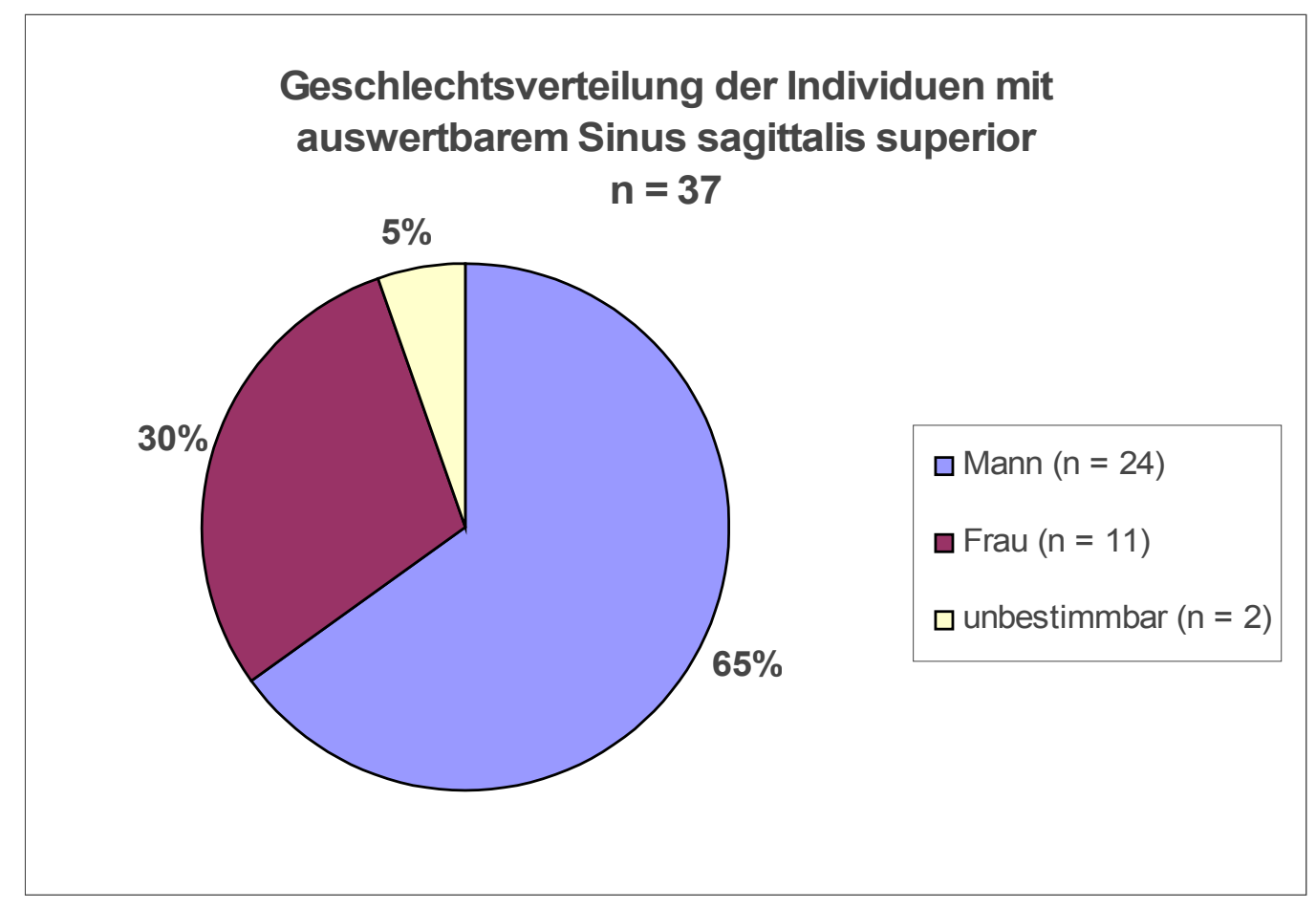

Abbildung 52: Geschlechtsverteilung der Individuen mit Sinus sagittalis superior

Ein gesunder Sinus besitzt eine glatte Oberfläche mit gelegentlichen feinlumigen Poren, in welche die Brückenvenen aus der Diploë münden. Als krank gilt ein Sinus, wenn die Oberfläche plattenartige Neubildungen oder fein poröse Platten zeigt. Dies weist auf eine entzündliche beziehungsweise hämorrhagische Genese der Läsion hin. Auch eine feinporöse Oberfläche des Sulcus des Sinus sagittalis superior gibt einen Hinweis auf pathologische Umbauprozesse. Hier ist anzunehmen, dass diese Oberfläche unphysiologisch stark vaskularisiert war. Eine Hypervaskularisation findet man bei Reizungen jeglicher Genese. Auch Abdrücke feiner Gefäße im Sinusbereich gelten als pathologisch, da diese Region bei Gesunden frei von Gefäßimpressionen ist.

Im Bereich des Sulcusrandes gelten die gleichen Kriterien. Beim Gesunden ist diese Region ein flacher und glatter Wulst. Bei perisinuösen Prozessen zeigt sich die Oberfläche je nach Art der Pathogenese wie zuvor beschrieben.

Für den Sinus sagittalis superior ergibt sich in der Baunacher Population eine folgende Verteilung (Abbildung 53): 


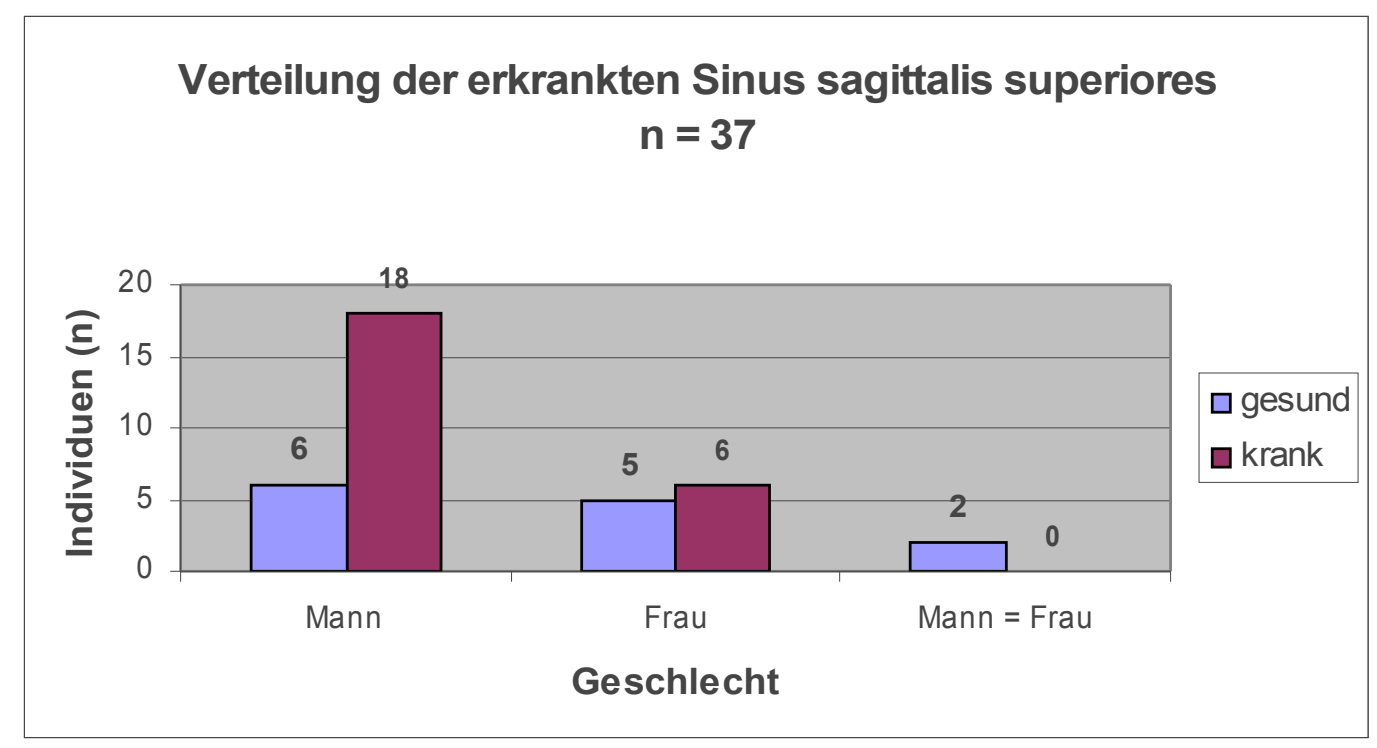

Abbildung 53: Geschlechtsspezifische Verteilung der erkrankten Sinus sagittalis superiores

Eine deutliche Häufung von pathologisch veränderten Sinus sagittales superior von 75 Prozent bei den männlichen Individuen ist zu erkennen. Diese Ergebnisse ähneln denen der Erkrankungshäufikeit der Lamina externa bei Männern (68 Prozent). Ein Zusammenhang zwischen extracranieller Entzündung der Kopfschwarte und erkrankten Sinus sagittales ist bei räumlich enger Nachbarschaft der Strukturen und gemeinsamen venöser Versorgung über die V. emissaria möglich.

Frauen zeigen dagegen ein ausgeglichenes Verhältnis zwischen krankhaft veränderten und gesunden Sinus.

Ein ebenso unausgeglichenes Verhältnis findet man auch bei der Aufteilung nach Läsionsort (Abbildung 54). Bei Männern ist der Sinusrand mit 61 Prozent häufiger als der eigentliche Sulcus des Sinus sagittalis superior mit 39 Prozent erkrankt. Hingegen weisen Frauen eine ausgewogene Verteilung zwischen erkranktem Sinusrand und Sinusfläche auf (jeweils 50 Prozent). Bei erkranktem Sinusrand spricht man von einer Perisinusitis. 


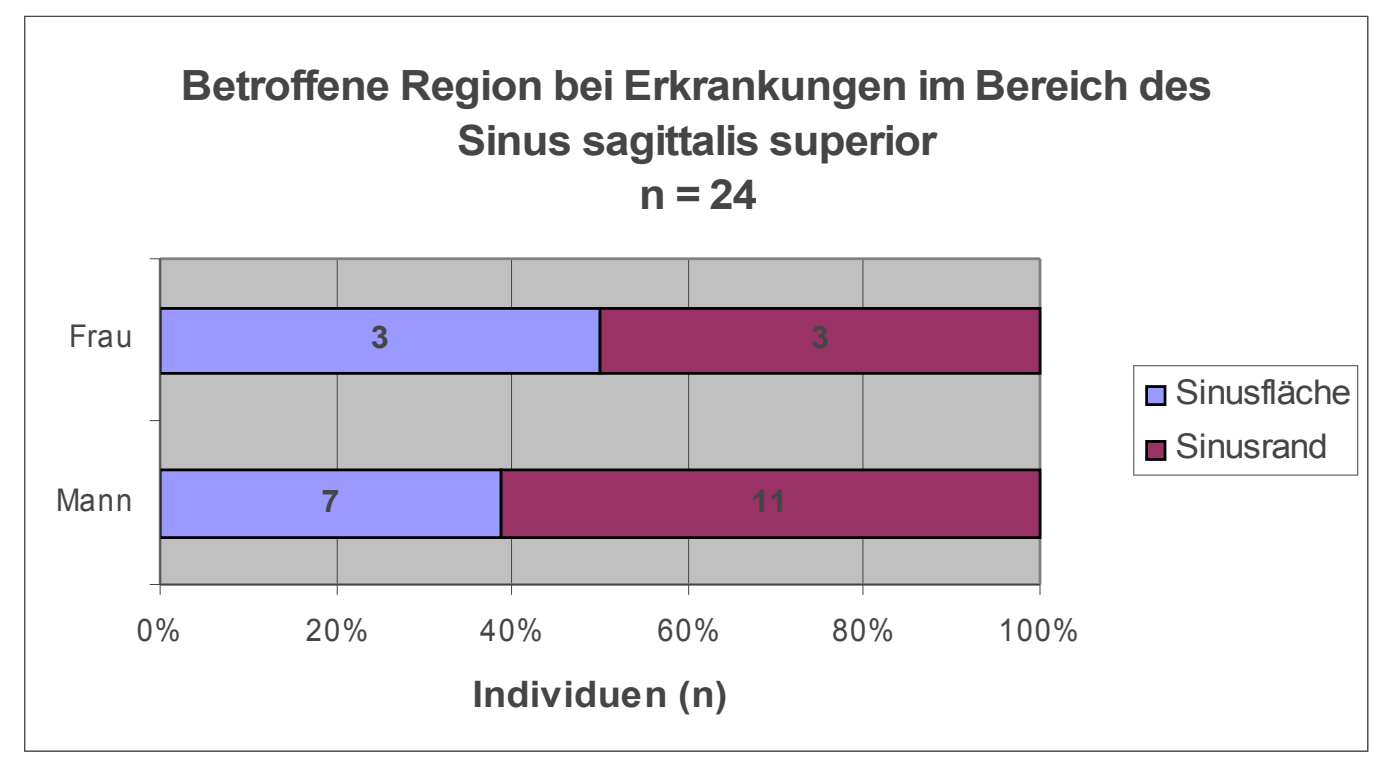

Abbildung 54: Geschlechtsspezifische Verteilung der betroffenen Regionen des erkrankten Sinus sagittalis superior

\subsubsection{Ergebnisse Sinus transversus}

Der Sulcus des Sinus transverus ist paarig und überwiegend auf dem rechten und linken Os occipitale angelegt.

Bei der Untersuchung der Sinus transversis konnten insgesamt 52 Sinus mit in die Berechnungen einbezogen werden. Hier musste wiederum zusätzlich zum Erhaltungszustand auch die makroskopische Befundungsmöglichkeit gewährleistet sein, was bei vollständig erhaltenen Schädeln oft nicht der Fall war.

Die Aufteilung der untersuchten Sinus transversi nach Geschlecht gibt folgendes Diagramm in Abbildung 55 wieder: 


\section{Verteilung der Sinus transversi

$$
\mathrm{n}=\mathbf{5 2}
$$

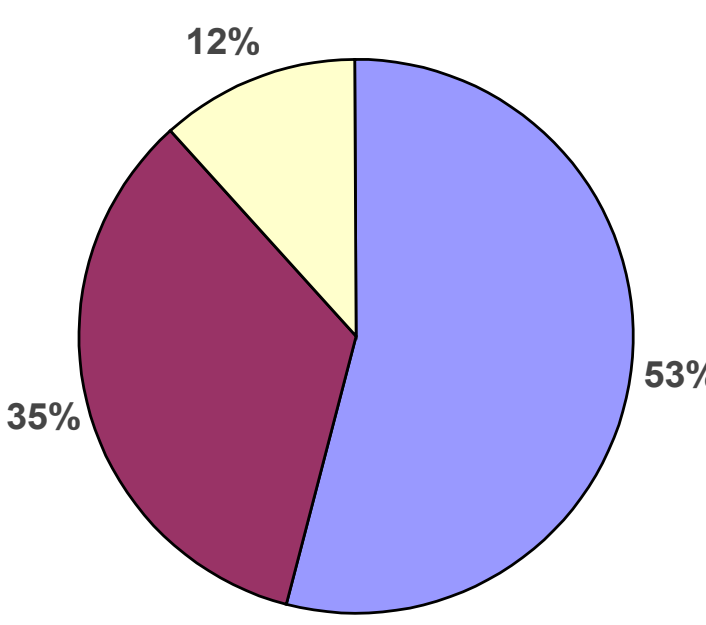

$\square$ Mann $(n=28)$

口Frau $(n=18)$

口unbestimmbar $(n=6)$

Abbildung 55: Geschlechtsverteilung der Individuen mit Sinus transversus

Es ist eine ähnliche Aufteilung wie schon beim Sinus sagittalis superior zu erkennen.

Bei der Untersuchung nach Erkrankungen des Sinus transversus, die schon beim Sinus sagittalis superior beschrieben wurden, stellt sich die in Abbildung 56 gezeigte Verteilung der Erkrankungen bei den Geschlechtern dar: 


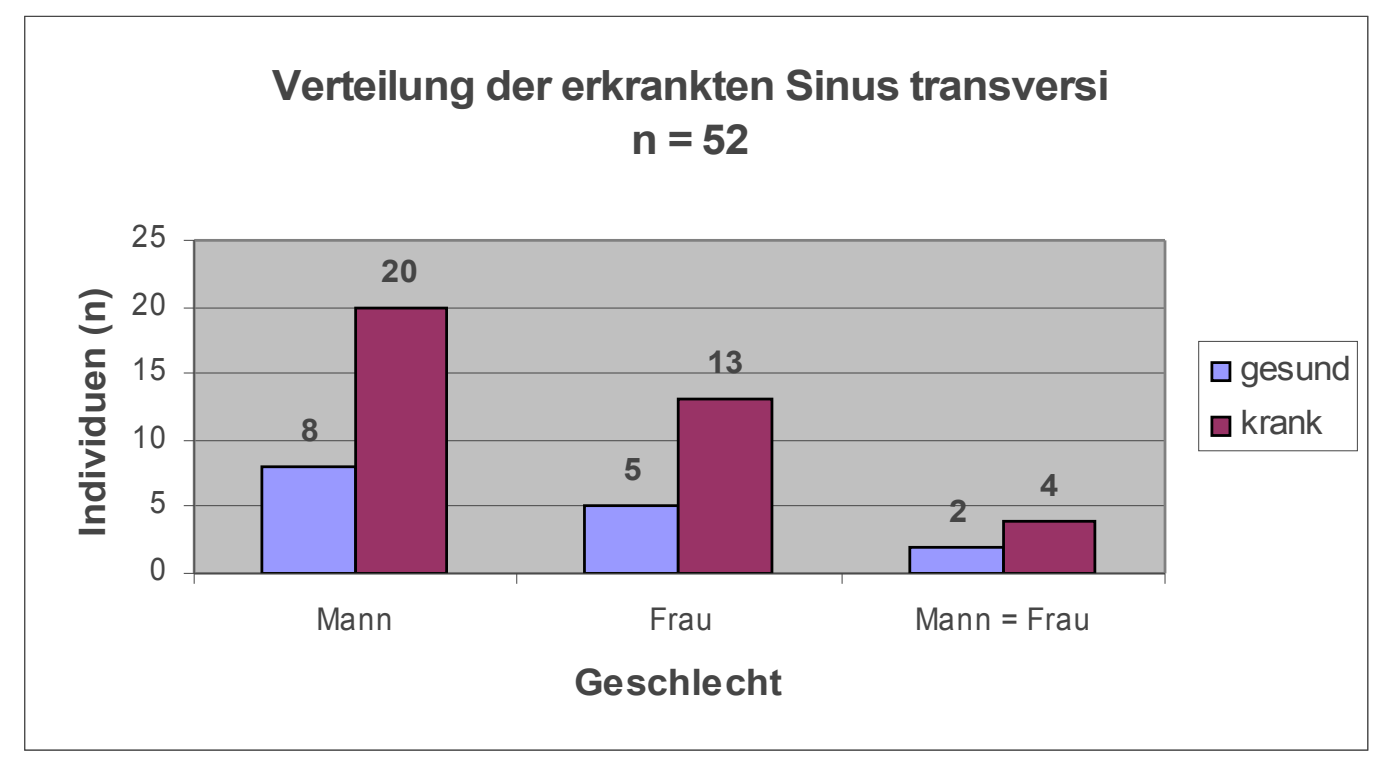

Abbildung 56: Geschlechtsspezifische Verteilung der erkrankten Sinus transversi

Auffällig ist hier, dass sich bei allen Geschlechtern eine annähernd gleiche Verteilung der Erkrankungshäufigkeiten ergibt. So sind 71 Prozent der Sinus transversi an männlichen Skleten krank, 72 Prozent bei weiblichen Individuen und 67 Prozent bei Schädeln mit nicht bestimmbarem Geschlecht.

Da nach verschieden Quellen eine Sinuserkrankung im Bereich des Sinus transversus mit einer Mastoiditis assoziert ist, liegt die Vermutung nahe, dass bei Personen mit den Zeichen einer Mastoiditis auch der korrespondierende Sinus transversus erkrankt sein sollte.

Die Beziehungen zwischen einer Mastoiditis und einer pathologischen Veränderung im Sinusbereich konnten an 40 Paaren untersucht werden. Für ein gewertetes Paar müssen jeweils das Mastoid sowie der Sinus transversus der entsprechenden Körperhälfte vorhanden und befundbar sein. Die Ergebnisse werden in Abbildung 57 gezeigt: 


\section{Zusammenhang zwischen krankhaft verändertem Sinus transversus und Mastoiditis

$$
\mathrm{n}=\mathbf{4 0}
$$
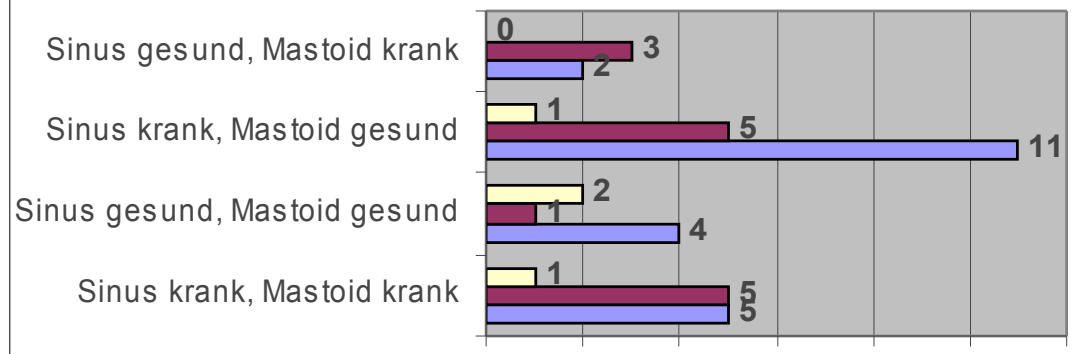

$\square$ Mann = Frau

\section{$\begin{array}{lllllll}0 & 2 & 4 & 6 & 8 & 10 & 12\end{array}$ \\ Individuen (n)}

Abbildung 57: Zusammenhang zwischen erkranktem Sinus transversus und Mastoiditis

Die erwarteten Häufungen von Paaren mit gesundem Sinus und gesundem Mastoid sowie krankem Sinus und krankem Mastoid lassen sich nicht feststellen. Diese Paarbildungen werden nur durch 45 Prozent der Gesamtpaare vertreten.

Die größte Gruppe mit 42 Prozent von der Gesamtmenge wird von Paaren gestellt, die einen kranken Sinus und ein gesundes Mastoid besitzen. Dagegen findet man Skelete mit zwei gesunden Strukturen nur zu 18 Prozent. Individuen, die einen kranken Sinus transversus und ein krankes Mastoid aufweisen sind mit 27 Prozent in der Gesamtmenge vertreten. Die kleinste Gruppe mit 13 Prozent wird von Paaren repräsentiert, bei denen der Sinus gesund aber das Mastoid krank ist.

Im folgenden Diagramm (Abbildung 58) ist diese Verteilung graphisch dargestellt: 


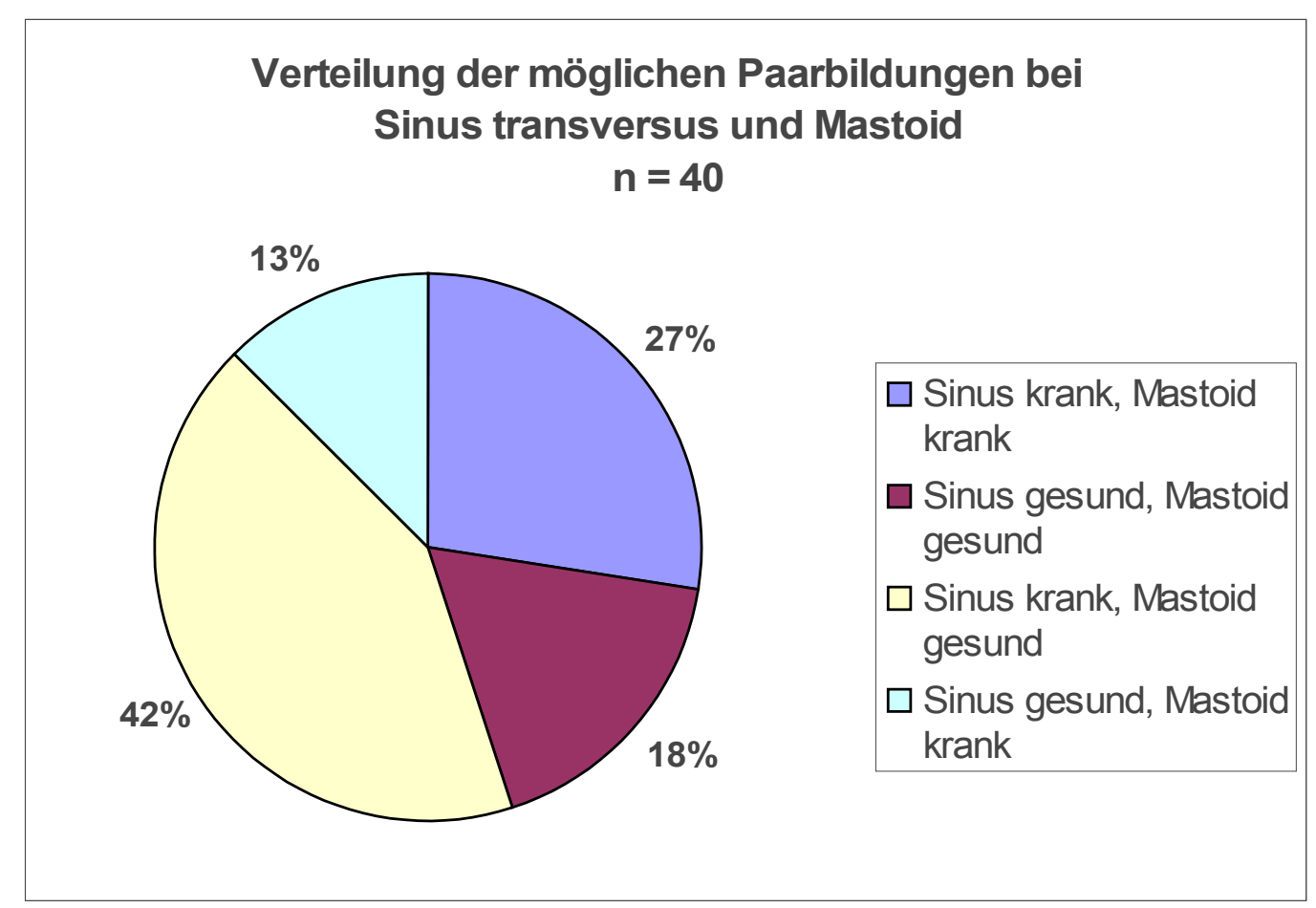

Abbildung 58: Verteilung der möglichen Paarbildungen bei Sinus transversus und Mastoid

\subsubsection{Ergebnisse Sinus sigmoideus}

Für Erkrankungen im Bereich des Sinus sigmoideus konnten 50 Sinus mit in die Bewertung einbezogen werden. Auch der Sinus sigmoideus ist beidseits im Bereich des Os temporale vorhanden. Es gelten die schon beim Sinus transversus beschriebenen Ein- und Ausschlusskriterien. In der Abbildung 59 wird die Geschlechtsverteilung der untersuchten Sinus sigmoidei dargestellt. 


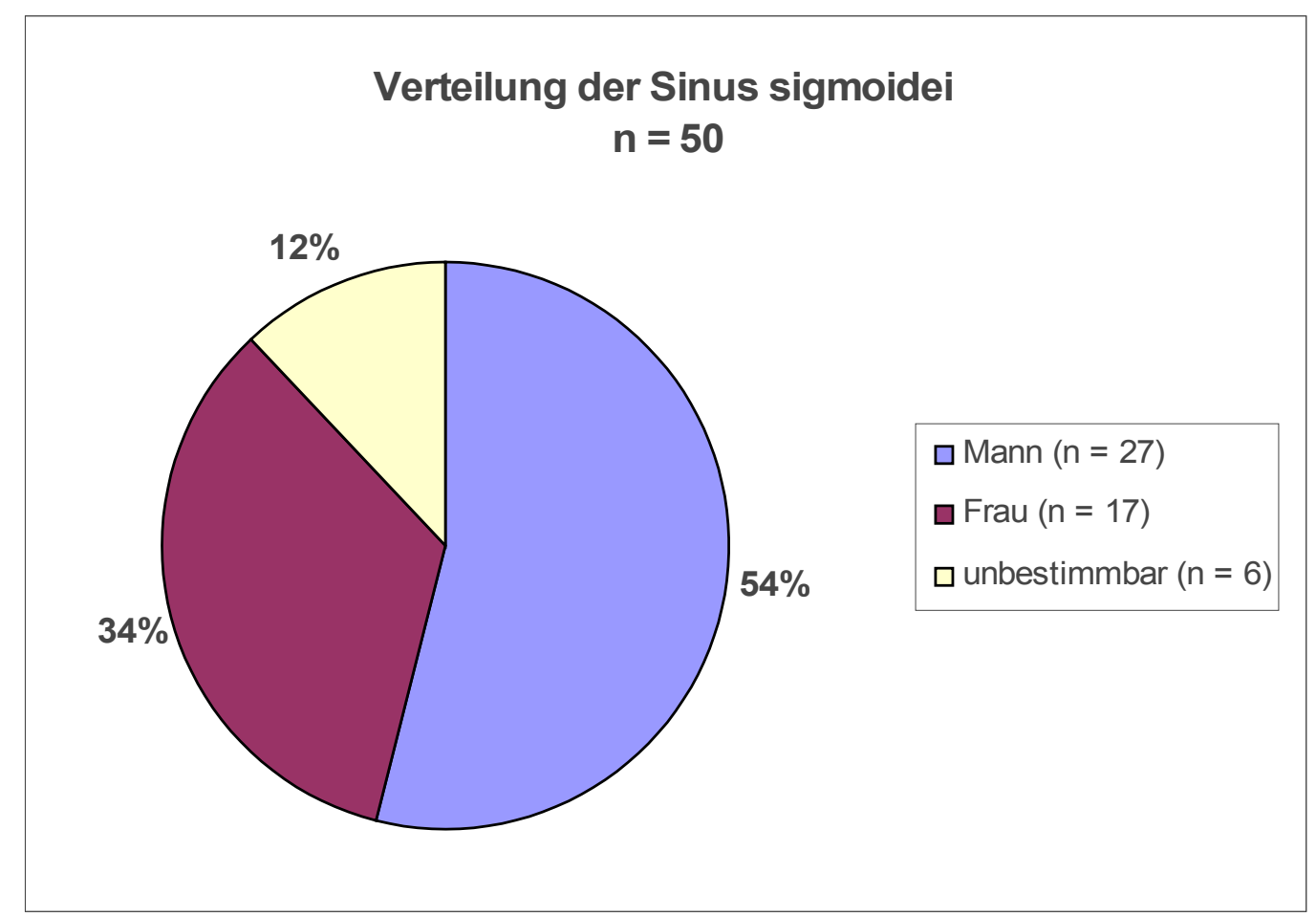

Abbildung 59: Geschlechtsverteilung der Individuen mit Sinus sigmoideus

Man findet eine fast mit dem Sinus transversus identische Verteilung.

Bei der Einteilung nach gesundem und kranken Sinus sigmoideus ergibt sich folgendes Bild (Abbildung 60):

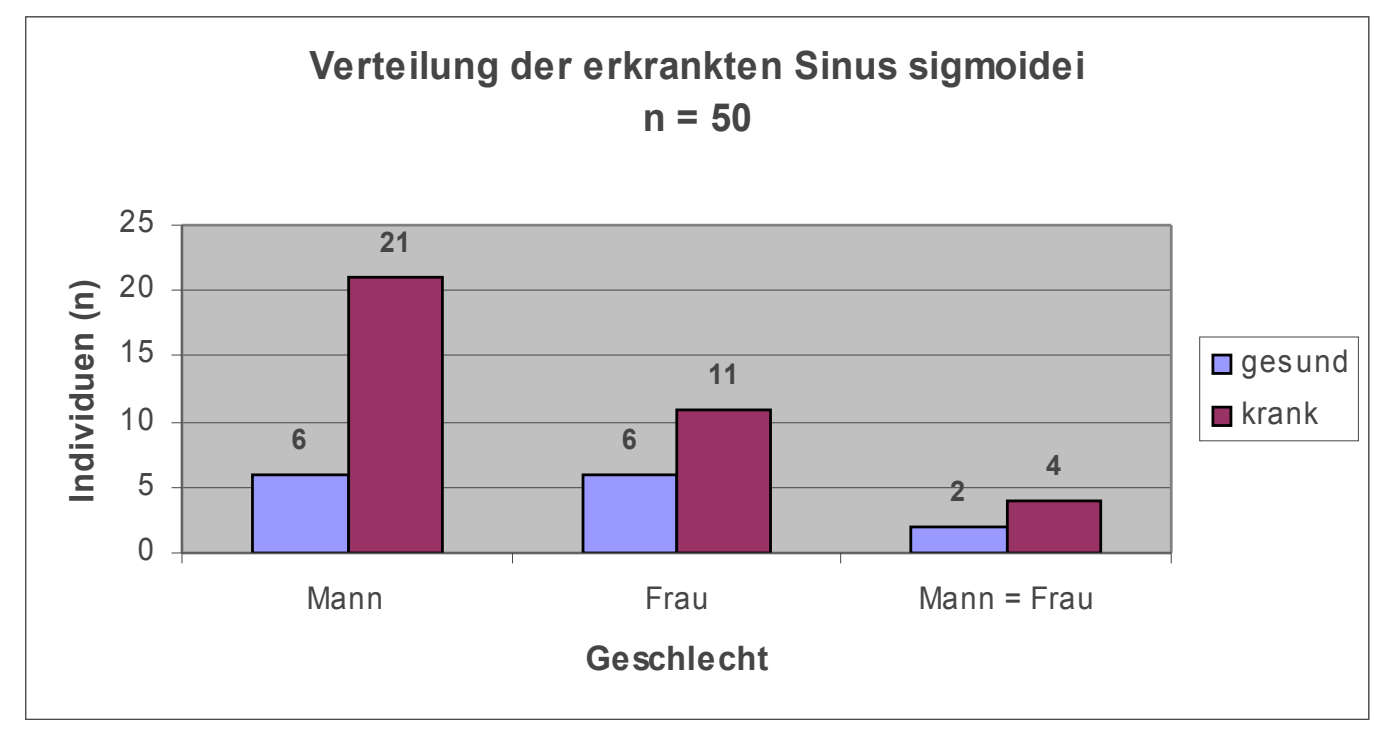

Abbildung 60: Geschlechtsspezifische Verteilung der erkrankten Sinus sigmoidei 
Männliche Individuen zeigen eine Erkrankungshäufigkeit von 78 Prozent. Bei Frauen stellt sich eine Häufigkeit von 65 Prozent dar. Individuen mit nicht zuordbarem Geschlecht sind zu 67 Prozent erkrankt.

Eine Schädigung des Sinus sigmoideus aufgrund seiner engen räumlichen Beziehung zum Mittelohr ist häufig mit einer Otitis media assoziiert. Bei der Untersuchung von Individuen mit einer Otitis media und dem korrespondierenden Sinus sigmoideus ergeben sich folgende Aufteilungen (Abbildung 61):

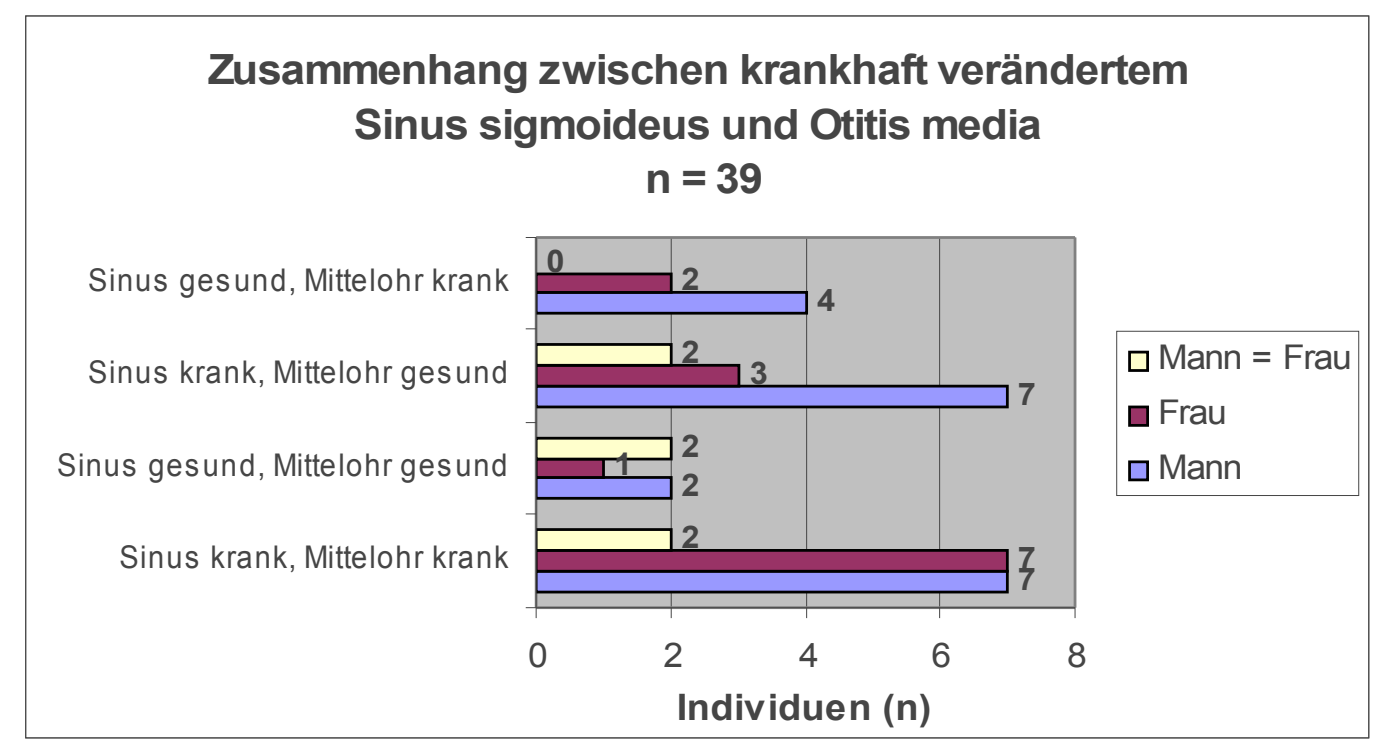

Abbildung 61: Zusammenhang zwischen Erkrankungen des Sinus sigmoideus und des Mittelohrs

Bei den Männern konnten 20 Individuen gefunden werden, bei denen der Sinus sigmoideus und das Mittelohr der gleichen Körperseite einer Befundung zugänglich sind. Von diesen 20 Individuen zeigen neun Personen Kombinationen, bei denen die beiden Strukturen gesund (zwei Paare) oder krank (sieben Paare) sind. Dies entspricht nur 45 Prozent der Gesamtmenge. Die größere Anzahl wird von Paaren gebildet, bei denen der Gesundheitszustand von Mittelohr und Sinus sigmoideus nicht gleich ist. Dabei zeigt die Mehrzahl (sieben Paare) der Männer einen kranken Sinus bei gesundem Mittelohr. Vier Paare weisen einen gesunden Sinus und ein krankes Mittelohr auf. 
Für Frauen bestätigt sich die Annahme, dass bei einer Mittelohrentzündung auch der Sinus sigmoideus krankhafte Veränderungen aufweist. Von insgesamt 13 Paaren können acht Paare übereinstimmende Zeichen an beiden Strukturen bieten (sieben Paare beide Strukturen krank, ein Paar beide Strukturen gesund). Dies entspricht 62 Prozent der Gesamtmenge. Drei Paare zeigen einen kranken Sinus bei gesundem Mittelohr. Bei zwei Paaren finden sich ein gesunder Sinus und ein krankes Mittelohr.

Bei Individuen der Gruppe Mann = Frau finden sich immer jeweils zwei Paare. Nur in der Gruppe Sinus gesund, Mittelohr krank lässt sich kein Paar einordnen. Insgesamt lässt sich für den Sinus sigmoideus folgende Verteilung (Abbildung 62) beobachten:

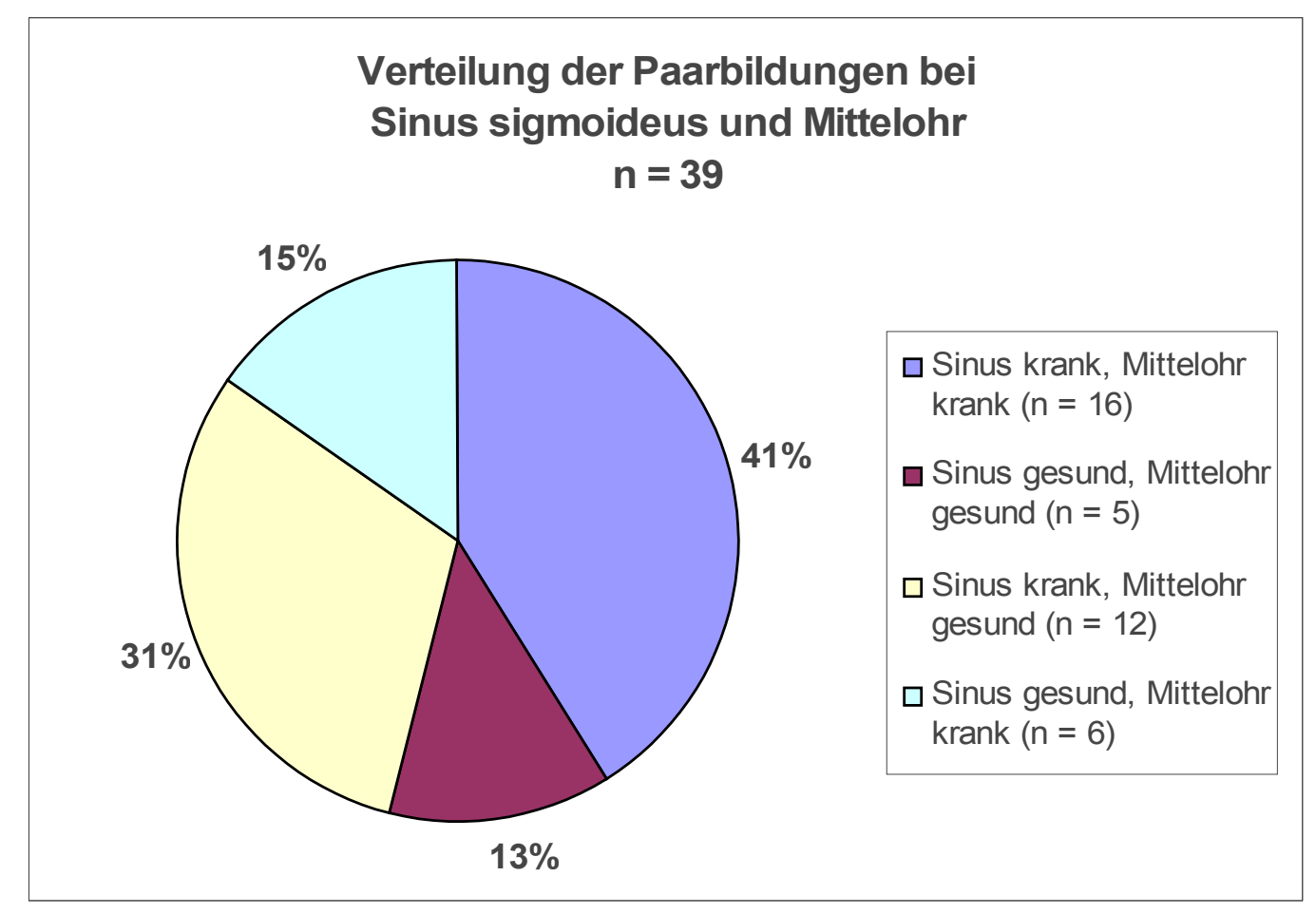

Abbildung 62: Verteilung der möglichen Paarbildungen bei Sinus sigmoideus und Mittelohr 
Hierbei zeigt sich, dass in der Mehrzahl der Fälle die oben genannte Vermutung zutrifft. Bei 54 Prozent der Gesamtpaare ist der Sinus sigmoideus und das korrespondierende Mittelohr jeweils mit dem gleichen Gesundheitszustand anzutreffen. Die Gruppe mit krankem Sinus und krankem Mittelohr macht dabei den größten Teil aus. Es kann vermutet werden, dassss bei einer Mittelohrentzündung durch hämatogene Infektionsausbreitung oder auch per continuitatem der sehr eng räumlich benachbarten Sinus sigmoideus ebenfalls Infektionszeichen ausbildet. Jedoch finden sich bei einer Otitis media nicht zwangsläufig auch ein erkrankter Sinus sigmoideus. Bei 15 Prozent der Proben sind bei sicherer Mittelohrentzündung keine entzündlichen Veränderungen im Sinusbereich zu erkennen. Die pathologischen Befunde im Bereich des Sinus sigmoideus sind also nicht nur auf Entzündungen im Mittelohr zurück zu führen. Bei 31 Prozent der Paare finden sich gesunde Mittelohren bei pathologischen Veränderungen im Bereich des Sinus sigmoideus.

\subsection{Pathologische Befunde in der Orbita}

\subsubsection{Anatomische Grundlagen}

Das Auge ist eines der wichtigsten Sinnesorgane des Menschen. Anders als bei vielen anderen Lebewesen liegt es daher zu einem großen Teil knöchern umhüllt im Schädel, um diese Aufgabe möglichst geschützt wahrnehmen zu können. Die Augenhöhle ist von der Periorbita ausgekleidet. Diese entspricht dem Periost.

Wie wir von der embryologischen Entwicklung des Auges wissen, sind die Netzhaut und der Nervus opticus ein Teil des Gehirns.

Da das Auge embryologisch ein Teil des Gehirns darstellt, ähnelt der Aufbau der Orbitawandschichten dem des Gehirns. Auch der Bulbus oculi ist von einer Art harten Hirnhaut, der Sklera, bedeckt. Diese Schicht kann, wie auch die Meningen bei endocraniellen Prozessen, am Knochen bei entzündlichen oder hämorrhagi- 
schen Reizungen über die Periorbita (Periost der Augenhöhle) pathologische Spuren hinterlassen. Im paläopathologischem Untersuchungsmaterial sind solche Zeichen häufig zu sehen.

\subsubsection{Erkrankungen der Orbita}

Die knöcherne Orbita ist bis auf den lateralen Anteil von Nasennebenhöhlen umgeben. Medial finden sich die Cellulae ethmoidales, kaudal der Sinus maxillaris, occipital der Sinus sphenoidales und rostral der Sinus frontalis.

Zwischen diesen pneumatischen Schädelräumen und der Orbita liegen teilweise nur sehr dünne knöcherne Lamellen. Es ist daher leicht verständlich, dass entzündliche Erkrankungen der Sinus paranasales auf die Orbita übergreifen können.

Orbitale Komplikationen eitriger Sinusititden sind bei fehlender antibiotischer Therapie klinisch häufig. Beispiele für eine klinisch bedeutende Komplikation sind die septische Thrombophlebitis und die Sinus-cavernosus-Thrombose als hämatogen streuende Erkrankung (UNSÖLD, GREEVEN 1997). Ein subperiostaler Abszess mit möglichem diffusem Eitereinbruch in die Orbita ist ein Beispiel für eine Infektion per continuitatem.

Die Knochenoberfläche ist beim gesunden Menschen glatt und nahezu frei von Gefäßimpressionen.

Bei Entzündungen des retrobulbären Raumes finden sich eine unregelmäßig geformte Oberfläche mit kleinen knöchernen Stippchen oder Einschmelzungen. Stippchen kann man zum Beispiel bei einer Orbitalphlegmone erwarten. Eine Orbitalphlegmone kann als Komplikation einer Sinusitis, eines Gesichtsfurunkel oder auch durch einen bakteriell infizierten Insektenstich verursacht werden. Einschmelzungen sind typisch für aktive Tuberkelgranulome. Fein poröse Auflagerungen und feine Gefäßimpressionen lassen einen hämorrhagischen Hintergrund der Läsion vermuten. 
Häufig am archäologischen Material sind auch Cribra orbitalia. Diese porösen Läsionen am Orbitadach können vor der histologischen Aufarbeitung als unspezifische Stressmarker angesehen werden. Im heutigen Sektionsgut findet man diese Veränderungen nur noch selten.

Auch im Falle von Anämie und einer dadurch gesteigerten Erythrozyten-Neubildung kann man Spuren davon am Orbitadach finden. Der Schädelknochen ist ein flacher Knochen und als solcher, ähnlich wie die Rippen oder die Wirbelkörper, zur Hämatopoese befähigt. Bei ausgeprägter und chronischer Anämie findet man ein verdicktes und fein poröses Orbitadach. Allerdings ist diese makroskopische Veränderung unspezifisch. Nur mit Hilfe einer mikroskopischen Untersuchung des Orbitadaches lässt sich die Diagnose einer Anämie durch eine typische Knochenstruktur sichern.

Die sichere Diagnose einer intravital entstandenen Läsion ist nur durch den Einsatz der Mikroskopie und Rasterelektronenmikroskopie möglich. An archäologischen Skeletfunden wird relativ häufig eine makroskopisch gestellte Diagnose "Cribra orbitalia“ durch die Ergebnisse der mikroskopischen Untersuchung revidiert. Die Bilder 11 und 12 im Bildanhang verdeutlichen einen Fall vom makroskopischen Verdacht zum rasterelektronischen Beweis der Verdachtsdiagnose.

\subsubsection{Ergebnisse Orbita}

Bei der Population aus Baunach sind 37 Individuen durch mindestens ein Orbitadach vertreten. Insgesamt sind 68 Orbitadächer vorhanden.

Die Verteilung nach Geschlechtern gestaltet sich folgendermaßen (Abbildung 63): 


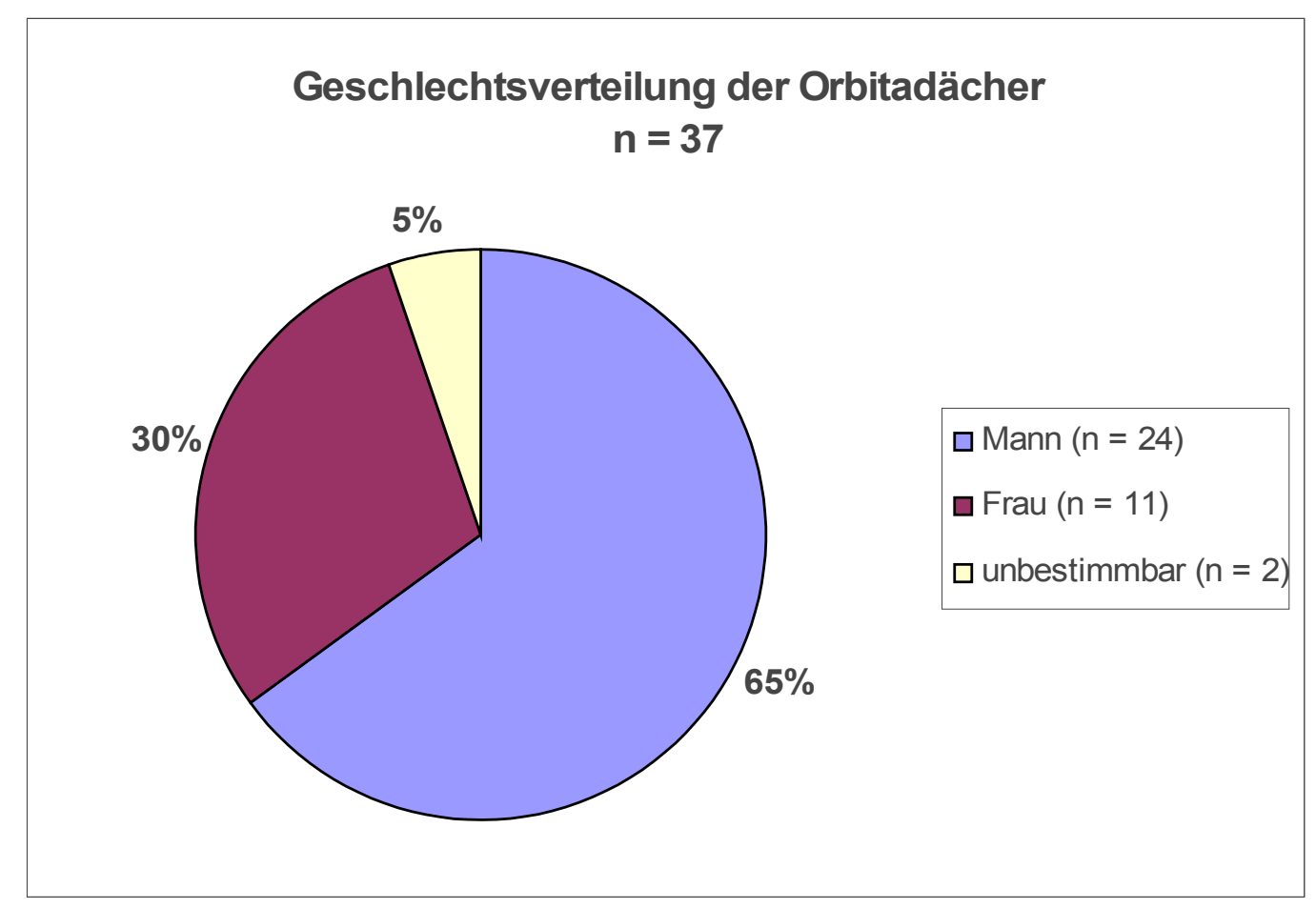

Abbildung 63: Geschlechtsverteilung der Individuen mit Orbita

Fast zwei Drittel der Stichprobe ( $\mathrm{n}=37$; 65 Prozent) sind Männer, fast ein Drittel sind Frauen $(\mathrm{n}=11 ; 30$ Prozent) und nur 5 Prozent $(\mathrm{n}=2)$ werden durch Individuen aus der Gruppe Mann $=$ Frau repräsentiert. Diese niedrige Zahl kommt dadurch zustande, dass bei erhaltener Orbita auch meistens auf ein Geschlecht geschlossen werden kann, da an diesem Schädelabschnitt relativ typische Geschlechtsmerkmale ab einem gewissen Alter ausgebildet sind. Fast alle Orbitadächer sind nicht normal glatt und zeigen Spuren pathologischer Prozesse. 


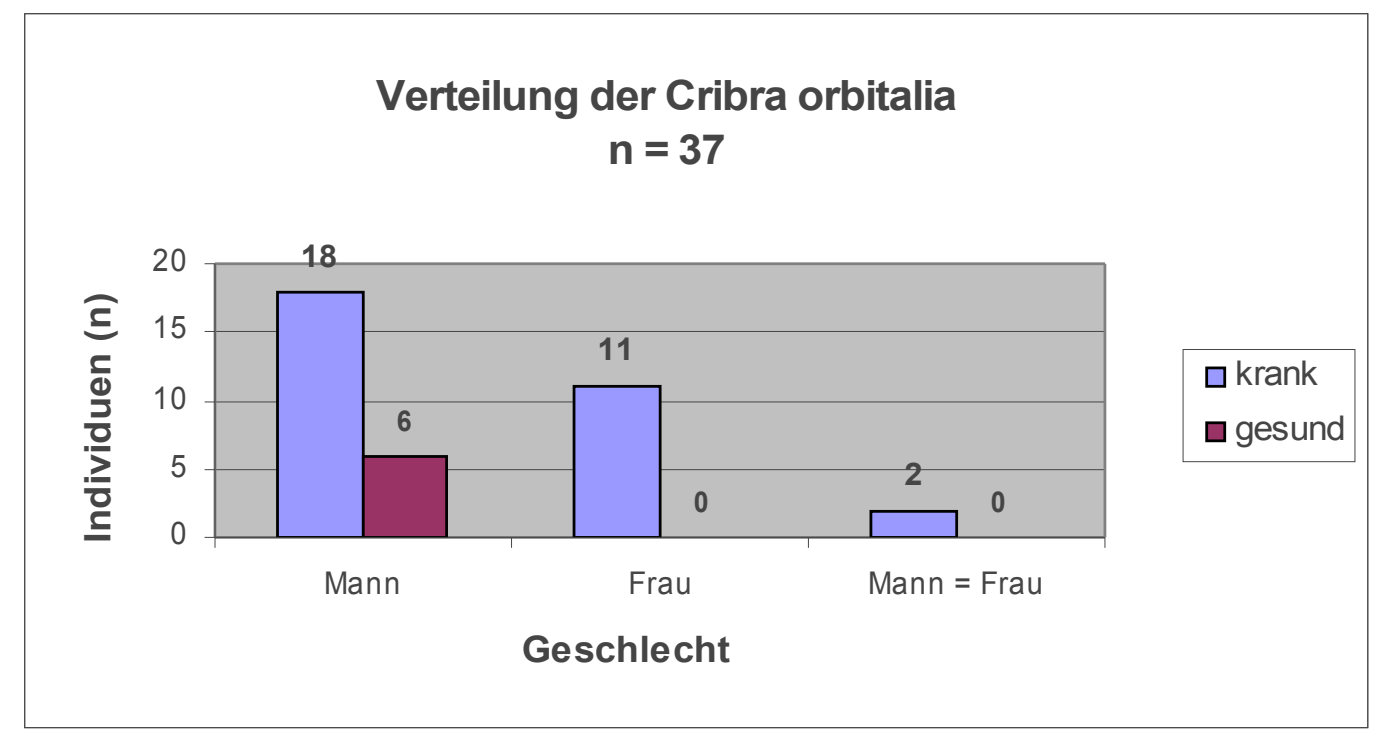

Abbildung 64: Geschlechtsspezifische Verteilung der erkrankten Orbitae

Es überwiegen die krankhaft veränderten Orbitadächer im Sinne einer Cribra orbitalia bei beiden Geschlechtern (Abbildung 64). Auffallend ist jedoch, dass es keine gesunden Orbitadächer bei den weiblichen Individuen gibt. Nach bisherigem Kenntnisstand ist die häufigste Ursache der Cribra eine durch Anämie verursachte Knochenmarksproliferation. Dieses bestätigt sich wohl auch in der Baunacher Population. Da alle weiblichen Individuen durch Menstruation und Geburten vermehrt Blut verlieren und somit ein größeres Anämierisiko besitzen, lässt sich diese Verteilung leicht erklären.

Jedoch kann diese Anämie bei der Baunacher Population nur einen Teil der Befunde erklären, da sich einige weibliche Individuen nicht in fertilen Lebensabschnitten befinden. Auffallend häufig finden sich auf Schädeln mit Cribra jedoch auch die Zeichen einer meningealen Tuberkulose. Eine chronische Entzündung als Ursache für die Cribra ist ebenso wahrscheinlich.

Bei weiterer Diagnostik kann man Unterschiede in der Morphologie der Cribraformen erkennen. Stark ausgeprägten Läsionen sind deutlich sichtbare eingeschmolzene Grübchen und mittel- bis grobporöse Streifen am Orbitadach. Kleinere Gefäßimpressionen und flache, scheinbar gut organisierte Neubildungen, die sich in der Regel mikroskopisch als vernarbte Läsionen $\mathrm{zu}$ erkennen geben, konnten nicht weiter diagnostiziert werden. 
Diese Kriterien erfüllen 11 Individuen (7 Männer $=64$ Prozent und 4 Frauen =36 Prozent). Auffällig ist, dass auf keinem Orbitadach beide morphologischen Ausprägungsvarianten zu sehen sind. Man findet bei vier Männern und drei Frauen einen porösen Streifen und bei drei Männern und einer Frau mindestens eine eingeschmolzene Grube (Abbildung 65).

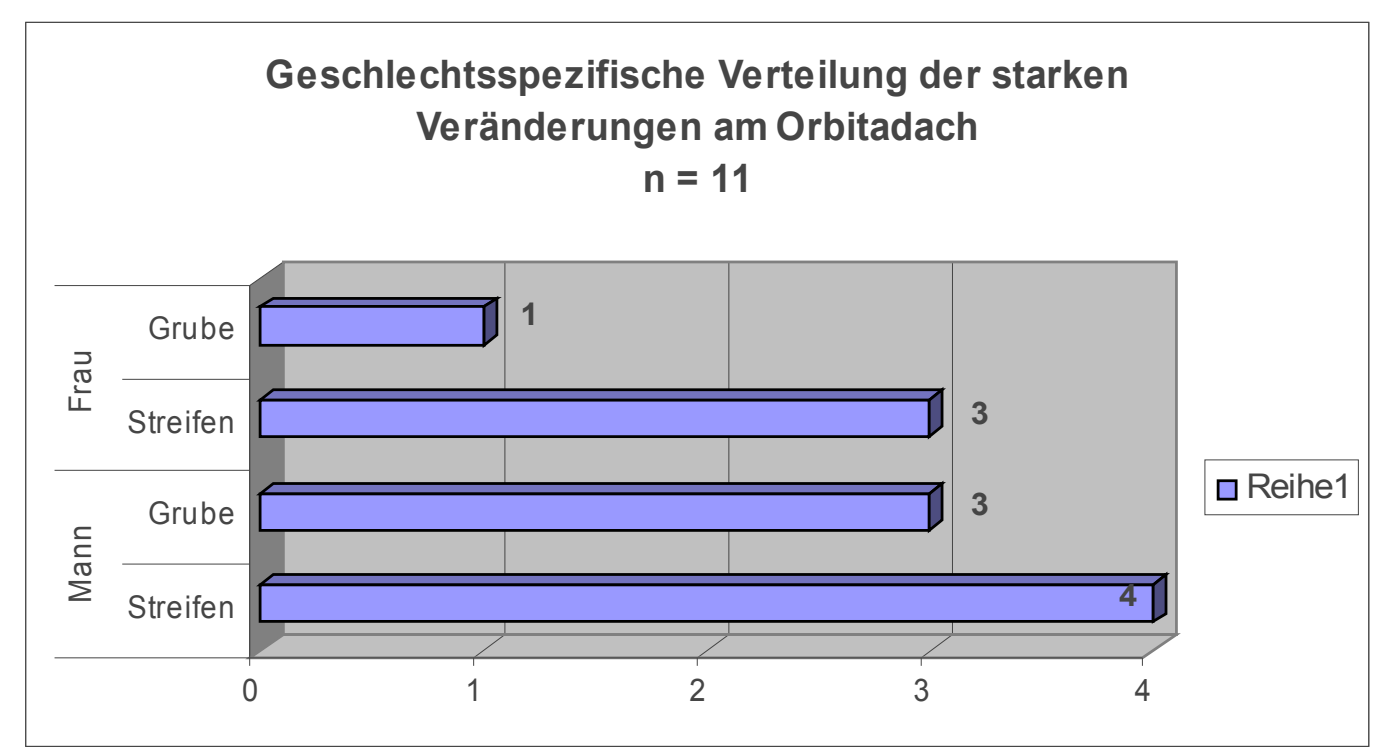

Abbildung 65: Geschlechtsspezifische Verteilung stark ausgeprägter Veränderungen am Orbitadach

In der aktuellen Literatur finden sich verschiedene Erklärungen für die Ausbildung einer Cribra (WAPLER 2004). In der Mehrzahl wird eine Anämie als Ursache angesehen. Die früher favorisierte Eisenmangelanämie als Hauptursache wurde in jüngster Zeit jedoch widerlegt (WALKER 2009). Ein Vitamin $B_{12}$ Mangel, ausgelöst durch Malabsorption oder Mangelernährung, wird aktuell als wichtigster Faktor für die Entstehung dieser morphologischen Auffälligkeit angesehen. Eine Malabsorption kann durch chronische Gastritis mit Mangel und Verlust von für die Resorption notwendigem intrinsic-Faktor verursacht werden. Häufige Nebenerkrankung bei einer chronischen TBC ist die unspezifische chronische Gastritis (GÜLZOW 1964). Man kann also vermuten, dass bei Personen mit Zeichen der TBC auch Cribra orbitalia häufiger zu finden sind. In der Baunacher Population zeigt sich eine starke Häufung der Cribra bei Individuen, welche auch 
die Zeichen einer chronischen intracraniellen Infektion bieten (Abbildung 66). Die Individuen zeigen in der Kombination „Cribra orbitalia“ und „endocranielle Infektion“ (chronische Leptomeningitis tuberculosa) eine große Übereinstimmung beider Merkmale. Bei der chronischen Infektion mit Tuberkeln findet man häufig eine chronische Anämie (ULRICI 1944) sowie eine chronische Gastritis als unspezifischen klinischen Hinweis. Der von WALKER (2009) favorisierte Vitamin $\mathrm{B}_{12}$-Mangel kann also theoretisch die Ursache der Cribra orbitalia sein. Aber auch entzündlich erscheinende Cribra sind häufig.

Jedoch kann ein durch gastrointestinale Infekte ausgelöster Vitamin $\mathrm{B}_{12}$-Mangel keine alleinige Erklärung der Cribra orbitalia sein. Wenn dem so wäre, würde im heutigen Sektionsgut ebenfalls eine Cribra häufig beschrieben werden. In einer Gesellschaft mit ausgeprägtem chronischen Alkoholgenuss und damit verbundener Magenschleimhautschädigung und chronisch atrophischer Gastritis findet man im klinischen Alltag sehr häufig einen Vitamin $\mathrm{B}_{12}$-Mangel. Theoretisch müssten nach WALKER also auch heute Cribra orbitalia häufig zu finden sein.

Die Erklärung von WAPLER mit mehreren möglichen Ursachen der Cribra ist als wahrscheinlicher anzunehmen.

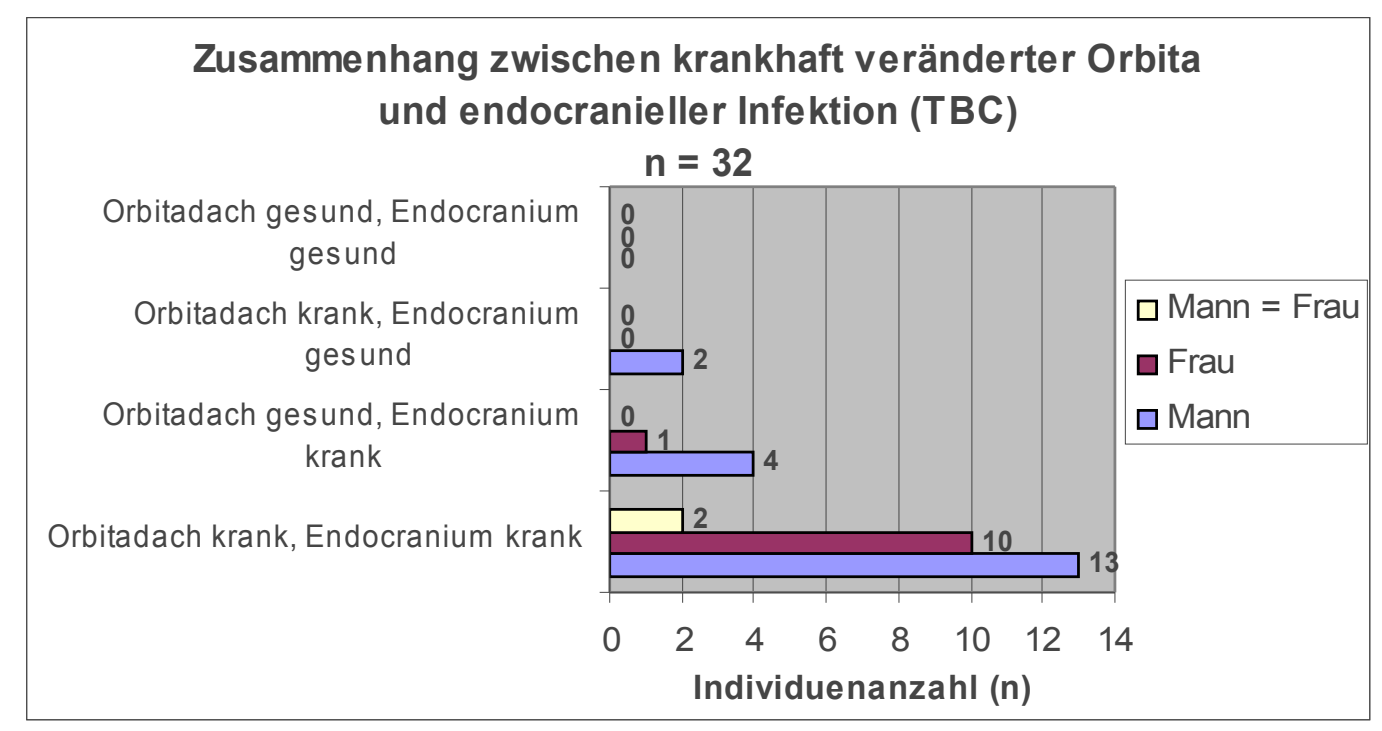

Abbildung 66: Zusammenhang zwischen Erkrankungen der Orbita und endocraniellen Infektionen 


\subsection{Pathologische Befunde am harten Gaumen}

\subsubsection{Anatomische Grundlagen}

Das Dach der Mundhöhle wird als Gaumen bezeichnet. Dieser gliedert sich in einen harten (knöchernen) und einen weichen Gaumen (Muskel- und Bindegewebeplatte). Am Skelet wird die Mundhöhle nur noch durch die knöchernen Abschnitte repräsentiert.

Der harte Gaumen wird von jeweils zwei paarigen Knochen (Os maxilla und Os palatina) gebildet, die sich über eine knöcherne Naht im Laufe des Lebens fest miteinander verbinden. Die knöchernen Strukturen im Bereich des harten Gaumens sind dünn und flach. Sie trennen den Raum der Mundhöhle (Cavitas oris) vom Raum der Kiefer- und Nasenhöhle (Sinus maxillaris et Cavum nasi). Wie die gesamte Mundhöhle sind auch diese knöchernen Begrenzungen von einer Schleimhaut bedeckt. Mit dem Knochen ist die Schleimhaut über straffe Bindegewebsfasern verbunden. Durch diesen ständigen Zug ist die Knochenoberfläche nicht glatt sondern zeigt viele kleine Höckerchen und Einkerbungen. Diese physiologisch unregelmäßige und fein poröse Oberfläche erschwert eine Befundung am archäologischen Material.

\subsubsection{Erkrankungen des harten Gaumens}

Die Schleimhaut der Mundhöhle steht als Ort des ersten Nahrungskontakts sowie beim Atmen und Sprechen ständig in Kontakt mit der Umwelt. Dadurch ist sie einer Vielzahl an exogenen und endogenen Einflüssen ausgesetzt. Bedingt durch diese möglichen Noxen entzündet sich die Schleimhaut bei insuffizienter Abwehr häufig. Ein unspezifischer Teil der Abwehr ist die Speichelproduktion. Dieses Produkt sero-muköser Drüsen ist reich an Enzymen, um Erreger schon im Mundraum abzuwehren. Zudem produziert die intakte Schleimhaut typische Immunglobuline, welche dem Körper schon bekannte Erreger binden und unschädlich machen. 
Beim Versagen dieser Schutzbarrieren entzündet sich zunächst die Schleimhaut. Bei länger dauernden Prozessen weitet sich der Prozess aber bis auf den Knochen aus. Dadurch wird das Periost gereizt und reagiert mit der Bildung von Faserknochen. Auch Gefäße sprießen ein und erhöhen damit die Porosität der Oberfläche. Bei hämorrhagischen Entzündungsreaktionen bilden sich bei der Organisation der Hämatome feinporöse Auflagerungen. Diese knöchernen Manifestationen lassen sich beim archäologischen Schädel untersuchen.

Die Ursachen für Stomatitis sind zahlreich. Erreger können aus der Umwelt aufgenommen (Bakterien in Nahrungsmitteln) oder eingeatmet (Viren, Bakterien) werden. Auch durch physikalische Noxen wie Wärme oder Kälte oder chemische Agenzien wird die Schleimhaut geschädigt und somit Eintrittspforte für Krankheitserreger. Häufig sind auch die von bakteriell infizierten Alveolen oder Zähnen ausgehenden Stomatitiden. Eine Mundschleimhautentzündung kann auch durch eine bestehende Sinusitis maxillaris und eine sich von dort per continuitatem ausbreitende Infektion verursacht werden (SEIFERT et al. 2000). Auch bei Mangelernährung und dabei im Speziellen ein Mangel an Vitamin C bilden sich Veränderungen am harten Gaumen aus. So manifestiert sich ein Skorbut bei Vitamin CMangel sehr häufig durch schlecht heilende Mundschleimhaut- und Zahnfleischgeschwüre (PASTINSZKY, RĂCZ 1965). Bei dieser Stomatitis gingivorrhagia findet man eine meist im frontalen Bereich beginnende typische Veränderung, die sich dann auf den restlichen Gaumenbereich ausdehnt. Um diese Erkrankung auszuprägen ist eine mehrere Monate dauernde Vitamin C - Avitaminose erforderlich (HEUSER 1971). Dies kann nach mehreren Wintermonaten in der Population Baunach ein durchaus zu erwägendes Problem gewesen sein. Bei dem zusätzlich anzunehmenden engen Kontakt mit Haustieren ist auch eine Stomatitis epidemica möglich. Dies ist eine meist von Paarhufern übertragene Viruskrankheit, die auch häufig mit entzündlichen Mundschleimhautmanifestationen einhergeht. 


\subsubsection{Ergebnisse harter Gaumen}

Für die Untersuchung des harten Gaumens konnten 39 Individuen herangezogen werden (Abbildung 67). Ein Individuum gilt als krank, wenn sich am harten Gaumen unspezifische Zeichen einer entzündlichen oder hämorrhagischen Erkrankung darstellen.

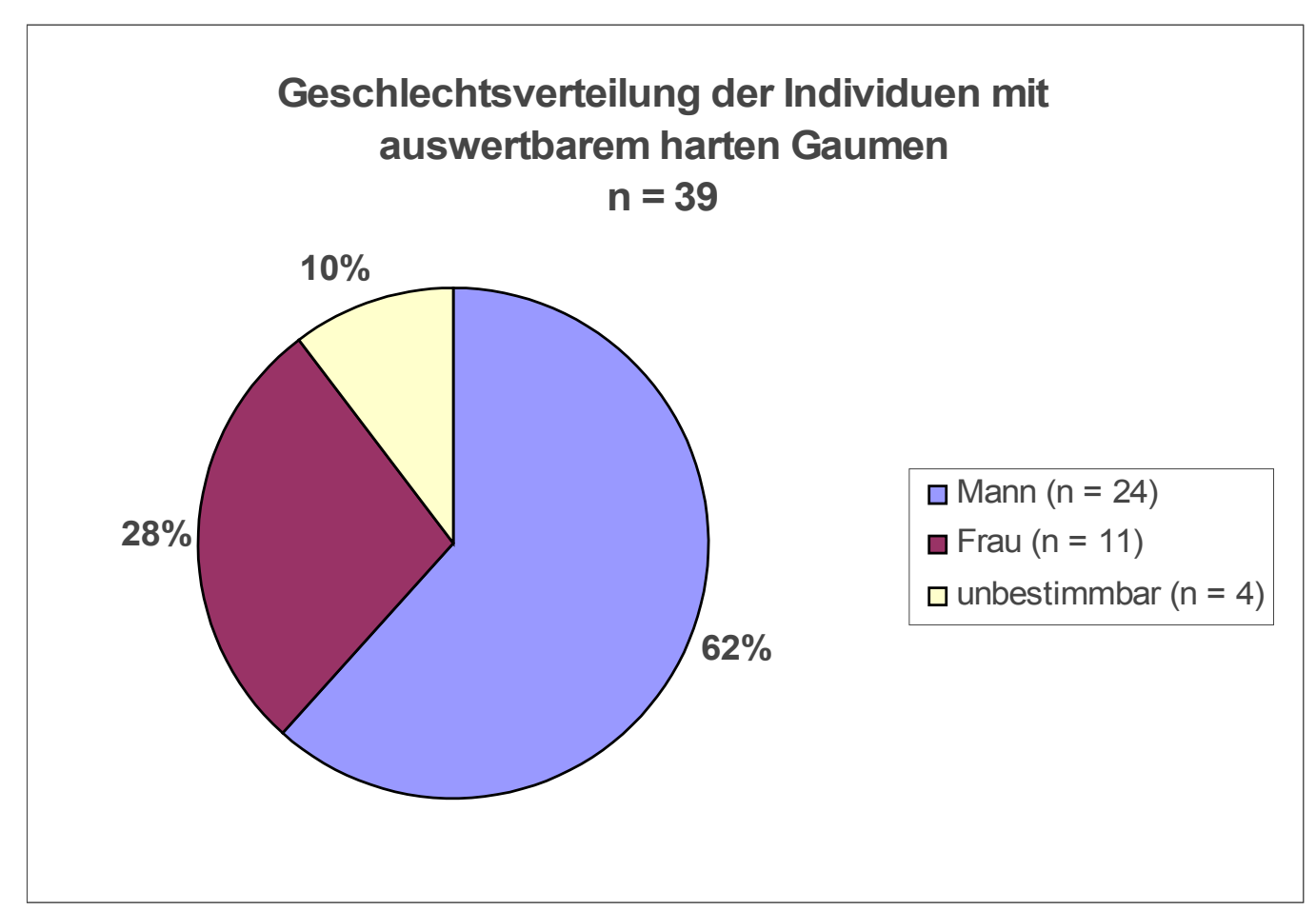

Abbildung 67: Geschlechtsverteilung der Individuen mit hartem Gaumen

Die Verteilung der Geschlechter fällt wie in den vorangegangenen Untersuchungen mit einer deutlichen Dominanz männlicher Skelete aus. 


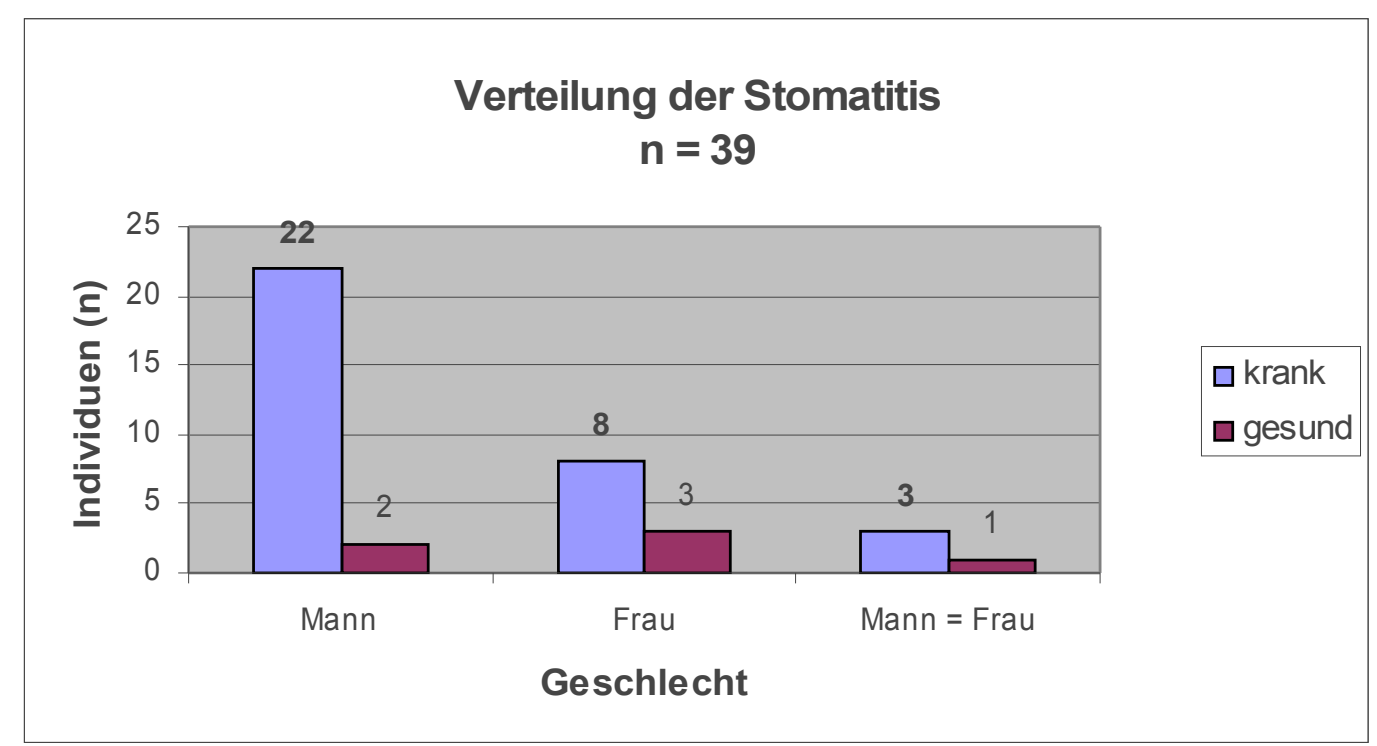

Abbildung 68: Geschlechtsspezifische Verteilung der Entzündungen des harten Gaumens

Ein Überwiegen von Individuen mit den morphologischen Zeichen einer Stomatitis wird deutlich (Abbildung 68). Dabei sind jedoch zwischen den Geschlechtern unterschiedliche Erkrankungshäufigkeiten festzustellen (Abbildung 69).

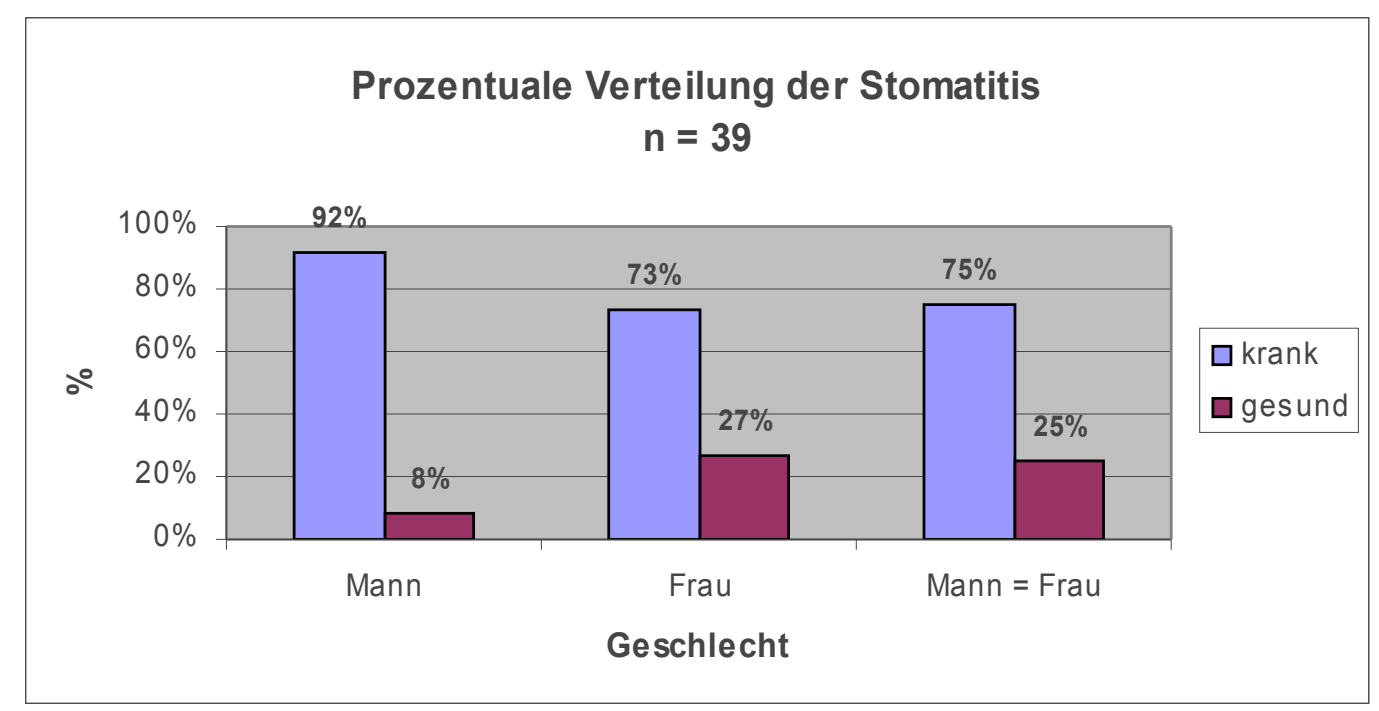

Abbildung 69: Geschlechtsdifferenzierte prozentuale Verteilung der Entzündungen des harten Gaumens 
Männer zeigen mit 92 Prozent weitaus öfter Läsionen am harten Gaumen, die für eine durchgemachte oder floride Stomatitis sprechen, als Frauen. Dagegen findet man bei Frauen und Kindern jeweils rund ein Viertel gesunde Individuen.

Häufig findet man bei der Untersuchung der harten Gaumen eine wulstig erhabene Knochenstruktur im Nahtbereich des harten Gaumens. Dies lässt häufig auf eine entzündungsbedingt veränderte Sutura palatina mediana schließen. In Folge dessen wuchert der Knochen durch einen chronischen Entzündungsreiz. Dieses Phänomen wird häufig mit den genetisch bedingten Torus palatinus verwechselt.

Das makroskopische Merkmal eines Torus palatinus findet sich in der Baunacher Population relativ häufig. Man findet dieses Merkmal in der Population Baunach jedoch nur bei Individuen, bei denen sich auch eine Stomatitis zeigt. Ein rein epigenetisch bedingter Torus palatinus kann nicht festgestellt werden. In Abbildung 70 wird die Verteilung der Individuen mit Torus palatinus dargestellt.

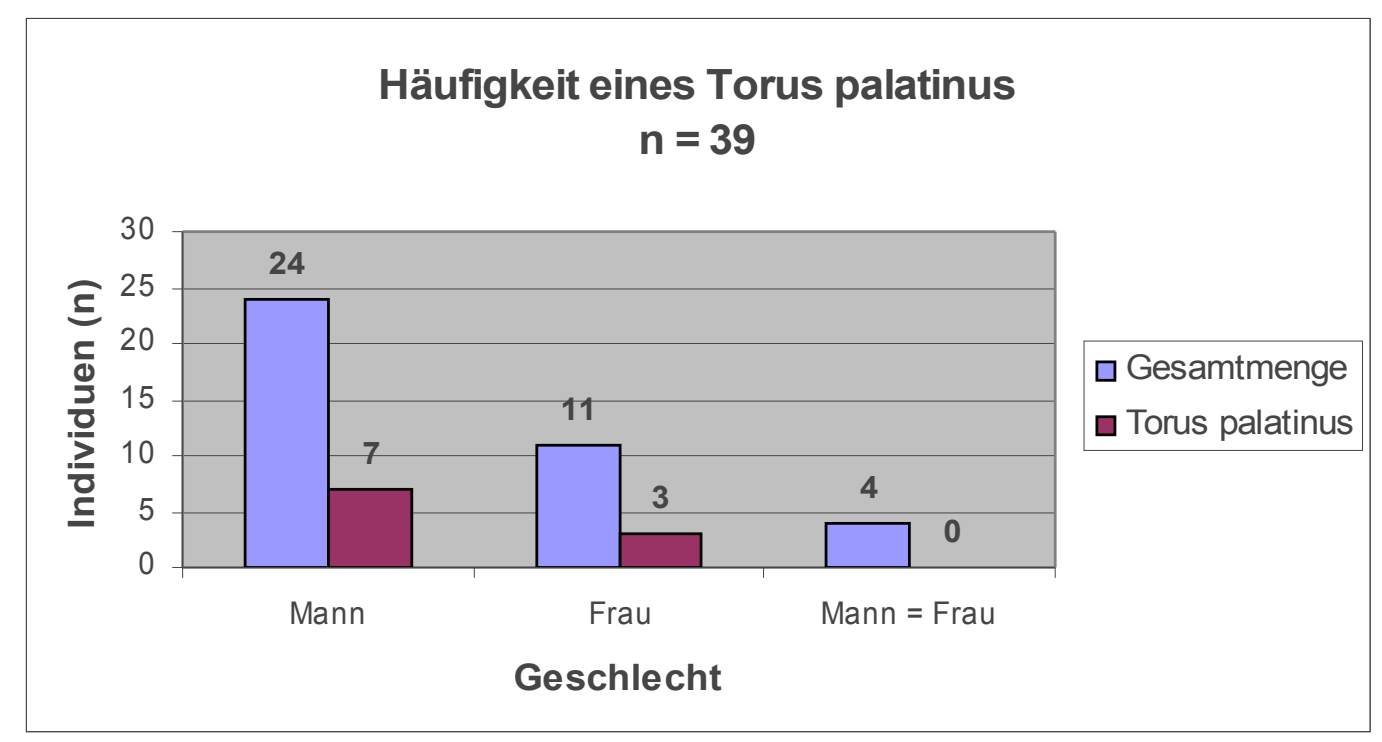

Abbildung 70: Geschlechtsspezifische Häufigkeit des Torus palatinus

Bei der prozentualen Auswertung ergibt sich für Männer und Frauen eine ähnliche Häufigkeit dieses wohl entzündlich bedingten Merkmals. Bei sieben der insgesamt 24 Männer (29 Prozent) sowie drei der elf Frauen (27 Prozent) ist ein Torus palatinus zu finden. Dagegen bietet in der Gruppe Mann $=$ Frau kein einziges 
Individuum dieses Merkmal. Sicher ist bei dem meist kindlichen Alters von Vertretern dieser unbestimmbaren Individuen und dem noch im Wachstum begriffenen Schädel ein Torus palatinus noch nicht ausgeprägt. 


\section{Diskussion}

Bei der Geschlechterverteilung der frühmittelalterlichen Population von Baunach zeigt sich mit 29 Männern und nur 15 Frauen ein deutlicher Männerüberschuß. Aufgrund des Mangels an sicher geschlechtsdiagnostizierbaren Individuen der Altersklassen Infans Ia \& Ib, Infans II sowie Juvenil werden dabei nur Individuen ab der Altersklasse Frühadult aufwärts in diese Berechnung einbezogen. Die Gründe für dieses Ungleichgewicht im Geschlechterverhältnis sind durch die paläopathologische Untersuchung nicht sicher zu bestimmen. Im Vergleich mit einer zeitlich vergleichbaren Untersuchung aus dem fünften bis siebten Jahrhundert aus dem süddeutschen Raum (GRESKY 2006: $\mathrm{n}=110$ mit 30 Prozent Männer, 37 Prozent Frauen; 33 Prozent Mann = Frau) ist der Männerüberschuß in Baunach auffällig.

Die Häufung männlicher Individuen in Baunach lässt sich durch mehrere Hypothesen erklären. Es könnten mehrere Individuen aus der Gruppe Mann = Frau weiblichen Geschlechts sein. Ein weiterer Grund ist möglicherweise der bei weiblichen Individuen grazilere Knochen, welcher durch Diagenese stärker zerstört werden kann und somit für die Geschlechtszuordnung wesentliche Knochenstrukturen in nicht verwertbarem Erhaltungszustand vorliegen. Diese Individuen zählten dann also irrtümlich in die Gruppe der nicht bestimmbaren Skelete.

Die Gründe für das gefundene Kinderdefizit sind ebenfalls spekulativ. Allerdings kann man räumlich getrennte Grablegungen nicht auszuschließen. Nur ein Teil des Gräberfeldes konnte bei der Grabung in Baunach als unzerstörter Grabungshorizont geborgen werden. Möglich ist eine Bestattung der Kleinkinder in der Siedlung beziehungsweise im Wohnhaus. Auch ein eventuell zu niedriger Grabhorizont bei Kindergräbern und damit verbundene Grabschädigung durch Überpflügen der Grabfelder in den folgenden Jahrhunderten kann für das Defizit verantwortlich sein. Sehr wahrscheinlich spielt auch die erhöhte Fragilität bei Kinderskeleten und die damit verbundene Erosion und Diagenese bei mehreren gefundenen leeren Kindergräbern eine entscheidende Rolle (DOLLHOPF 2002). In einem 
schnell wachsenden kindlichen Knochen ist der organische Anteil im Verhältnis zum ausmineralisierten Knochen des Erwachsenen sehr viel höher. Daraus resultiert eine höhere Geschwindigkeit des diagenetischen Knochenabbaus kindlicher Skelete im Boden (LEWIS 2000) und ein mögliches Kinderdefizit in der paläopathologischen Auswertung der Population Baunach.

Ein möglicher Grund für die gesteigerte Lebenserwartung männlicher Individuen ist das Fehlen von männlichen Kindern und Kleinkindern in dieser Stichprobe. Auch fehlen bei Männern die Schwangerschafts- und Geburtskomplikationen, welche die durchschnittliche Lebenserwartung der Frauen deutlich reduzierten.

An Hand der anthropologischen Altersbestimmungen der Baunacher Individuen zeigt die errechnete Lebenserwartung einen deutlichen geschlechtsspezifischen Unterschied. So fällt auf, dass ein Mann mit großer Wahrscheinlichkeit ein relativ hohes Lebensalter erreichte, sobald er die kritische Phase des frühadulten Lebensalters überlebt hatte.

Ein möglicher Grund für die hohe Sterbewahrscheinlichkeit der Männer im frühadulten Lebensabschnitt ist der Einsatz als Krieger. Trotz der zu damaliger Zeit schon lange zurückliegenden Völkerwanderung und der eingetreten Sesshaftigkeit der Menschen in der Zeit von 750 - 900 n. Chr. fanden kriegerische Auseinandersetzungen sehr wahrscheinlich relativ häufig statt und werden auch ihren Tribut in Form von Kriegerleben gefordert haben. Andererseits sind an keinem Schädel der Verstorbenen eindeutige traumatisch bedingte pathologische Befunde erhoben worden, die einen Hinweis auf Verletzungen durch Waffengewalt geben. Lediglich kleinere intravitale Corticalisverletzungen des Schädeldaches im Sinne einer Bagatellverletzung wurden nachgewiesen. Der Einsatz als Soldaten der männlichen Baunacher Individuen ist unwahrscheinlich.

In der Population Baunach stellen die Frauen im gebärfähigen Alter den größten Anteil der Verstorbenen dar. In der Regel ist die Altersphase zwischen 20-29 Jahren für Frauen mit den meisten Geburten belegt. Diese stellen ein hohes Risiko für Infektionen oder andere schwere Schwangerschaftskomplikationen dar, welche damals nicht selten den Tod der Mutter nach sich zogen. Aber auch im juvenilen Alter von 15 bis 20 Jahren sowie im spätadulten Alter von 30 bis 40 Jahre sind 
Schwangerschaften und Geburten sicher häufig für den Tod der Frauen verantwortlich gewesen. In diesen drei Altersklassen sind auch die meisten Baunacher Frauen verstorben (Abb. 10), so dass Fertilität und damit verbundene Geburten als ein Risikofaktor für einen frühen Tod im Frühmittelalter angesehen werden kann. Rund zwei Drittel der Frauen verstarben in diesen fertilen Altersklassen.

Weiterhin ist auffällig, dass der Anteil der im senilen Alter verstorbenen Frauen mit 6,6 Prozent nur etwa halb so groß wie bei den Männern (11 Prozent) ist. Daraus und aus der insgesamt mit zehn Jahren höheren Lebenserwartung der Männer zum Zeitpunkt der Geburt kann man schließen, dass entweder Männer insgesamt gesünder lebten beziehungsweise über günstigere Lebensbedingungen verfügten oder aber viele Frauen bei Schwangerschaften und den daraus entstehenden Komplikationen verstarben.

Eine erhöhte Sterblichkeit weiblicher Kinder aus sozialen Gründen, wie bei Kulturen aus dem asiatischen Raum bekannt, ist unwahrscheinlich.

Die paläopathologischen Untersuchung an den erhaltenen Schädeln der Individuen aus Baunach ergab eine Vielzahl auffälliger Befunde.

Sehr häufig finden sich Spuren chronisch entzündlicher Prozesse. Dies trifft sowohl für den Hirnschädel als auch für den Gesichtsschädel zu. Dabei zeigen sich sehr ähnliche Erkrankungshäufigkeiten bei Männern und Frauen im Bereich der Nasenhöhle und der Nasennebenhöhlen. Die Erkrankungshäufigkeiten in diesem Abschnitt des Gesichtsschädels sind sehr hoch (90-100 Prozent). In vergleichbaren Populationen ist diese Häufigkeit jedoch nicht ungewöhnlich (GRESKY 2006). So wird in der frühmittelalterlichen Population Harting (Oberpfalz) die Rhinitishäufigkeit erwachsener Individuen mit 95 Prozent angegeben.

Die mit 67 Prozent geringere Häufung der nachgewiesenen Rhinitis in der Gruppe geschlechtlich nicht einzuordnender Individuen ist möglicherweise durch das geringere Alter der Individuen dieser Gruppe bedingt. Wie schon zuvor erwähnt, stellen diese Kategorie hauptsächlich Vertreter der Gruppen Infans I bis Infans II. In diesem Alter organisiert sich ein kindlicher Knochen schneller als der eines Erwachsenen. So hinterlassen eventuell schon einmal durchgemachte und verheilte Nasenhöhleninfektionen bei Kindern, die zum Zeitpunkt des Todes nicht 
mehr an Rhinitis erkrankt waren seltener Spuren als bei Erwachsenen, bei denen sich der Knochen langsamer organisiert und ausheilt. Jedoch ist die knöcherne Manifestation einer Erkrankung schneller ausgebildet als bei adulten Individuen.

Eine andere Erklärung könnte die stärkere Belastung der Erwachsenen mit häufigerem Aufenthalt im Freien und damit steigender Infektionsrate sein. Auch könnte es möglich gewesen sein, dass Kinder einen hohen Stellenwert in der Gesellschaft inne hatten und durch eine bessere Ernährung auch ein leistungsfähigeres Immunsystem besaßen. Wie schriftliche Quellen aus dem Frühmittelalter berichten, besaßen Kinder jedoch keine herausragende Rolle in der Gesellschaft (ETTER 1982), so dass diese Überlegung abzulehnen ist. Durch den nur geringen Stichprobenumfang sind diese Ergebnisse allerdings nicht pauschalisierbar und stellen eventuell nur eine Tendenz dar.

Aus mehreren vorhergehenden Untersuchungen (SCHLOMM 2000, GRESKY 2006, ROUMELIS 2007) ist bekannt, dass Erkrankungen in den Nasennebenhöhlen als pneumatischer Teil des Gesichtsschädels sehr häufig auftreten. Dies ist unter anderem sicher auf hygienische und klimatische Verhältnisse (siehe 3.3.2) zurückzuführen. Eine sichere Unterscheidung in Eintrittspforte mit primären Entzündungsherd und nur fortgeleitete Entzündungsreaktion kann man bei zumeist mehreren weiteren entzündlichen Foci am Schädel jedoch nicht vornehmen.

In der Population Baunach haben 94 Prozent der Männer und 100 Prozent der Frauen eine floride oder in Organisation begriffene Sinusitis. Diese sehr große Häufigkeit einer Erkrankung erscheint im Vergleich mit rezenten mitteleuropäischen Populationen $\mathrm{zu}$ hoch. Jedoch weisen vergleichbare paläopathologische Untersuchungen einer frühmittelalterlichen Population in Mitteldeutschland (DETKEN 1991, ROUMELIS 2007, GRESKY 2006) mit jeweils 71 Prozent, 78 Prozent und 96 Prozent erkrankten Kieferhöhlen ähnliche Befunde auf. Da Infektionen der Nasennebenhöhlen häufig chronisch verlaufen und diese chronischen Infektionen eine kontinuierliche Erregersträuung in das respiratorische System verursachen, werden pulmonale Infekte die Folge solcher Sinusitiden gewesen sein. Jedoch ist auch eine chronische pulmonale Infektion als Ursache der starken Häufung von Erkrankungen der Nasennebenhöhlen denkbar. 
Es fällt auf, dass beide Geschlechter eine annähernd gleiche Verteilung bei dem Ausprägungsgrad einer Sinusitis maxillaris aufweisen und keine geschlechtsspezifische Häufigkeit zu erkennen ist. Die Verhältnisse bei der nicht geschlechtsbestimmbaren Gruppe lassen sich mit der Altersverteilung innerhalb dieser Gruppe erklären. Durch das geringere Alter und das schnellere Wachstum werden durchlebte Erkrankungen und dadurch verursachte pathologische Veränderungen offenbar schneller wieder integriert und somit erscheinen die Individuen makroskopisch gesund.

Männer und Frauen weisen mit 100 Prozent Erkrankungshäufigkeit den höchstmöglichen Wert für das Auftreten einer Sinusitis frontalis auf. Nur ein geschlechtlich nicht bestimmbares Individuum zeigt einen gesunden Sinus frontalis. Dies ist bei gehäuftem Auftreten einer Sinusitis maxillaris sowie häufigen intracraniellen Entzündungen nicht unwahrscheinlich. Allerdings gibt es die Möglichkeit einer zu stark pathologisch ausgerichteten Befundung des erhaltenen Skeletmaterials. Ohne Zerstörung ist bei komplett erhaltenem Cranium und vollständig verknöcherten Knochennähten eine vollständige Untersuchung aller intracraniell gelegenen Strukturen nicht möglich. Ein Individuum mit komplett erhaltenem unzerstörtem Cranium kann gesund oder krank sein. Eventuell sind Schädel gesunder Individuen von stabilerer knöcherner Struktur und damit widerstandsfähiger gegenüber Erosion und Diagenese. Somit könnten viele der Individuen mit komplett erhaltenem Schädel der Population Baunach gesunde Stirnhöhlen besitzen und somit die Statistik zu Gunsten der Gesundheit ändern. Durch die geringe Stichprobenzahl ist dieses Ergebnis jedoch nicht repräsentativ. Tendentiell muß bei der Vielzahl an weiteren Infektionszeichen im einsehbarem Bereich der Schädel aber von einer erkrankten Stirnhöhle ausgegangen werden.

Man findet vergleichbare Prozentzahlen der Erkrankungshäufigkeit auch bei der Sinusitis maxillaris. Dort ist der Stichprobenumfang mit $n=31$ (erkrankt $n=$ 29/30; 94 Prozent) jedoch fast doppelt so groß wie bei der Sinusitis frontalis (erkrankt $\mathrm{n}=16 / 17$; 94 Prozent). Auch diese Erkrankung kann durch weitere oral gelegene mögliche Infektionsquellen, wie Stomatitis, Karies oder Abszesse, begünstigt werden. Bei geringem Umfang der Stichprobenmenge der Stirnhöhlen $(\mathrm{n}=15)$ ist bei dieser großen Erkrankungshäufigkeit davon auszugehen, dass 
auch bei dieser kleinen Zahl an verwertbaren Stirnhöhlen fast jedes Individuum in der Population Baunach unter einer chronischen oder akut exacerbierten chronischen Sinusitis frontalis litt beziehungsweise diese zum Todeszeitpunkt gerade in Abheilung begriffen war.

Bei der Vielzahl an möglichen Komplikationen einer chronischen oder fortgeschrittenen Sinusitis ist diese Erkrankung sehr ernst zu nehmen. In Zeiten vor der Entdeckung der Antibiotika werden diese Infektionen mit möglicher Sepsis und den sich daraus ergebenden Komplikationen an lebenswichtigen Strukturen sogar eine wesentliche Todesursache für die damaligen Menschen dargestellt haben.

Bei den untersuchten Mittelohren findet man ebenfalls eine Häufung von entzündlichen Erkrankungen. Es zeigt sich erwartungsgemäß eine große Zahl an entzündlichen Veränderungen am Mittelohr in der Population von Baunach. Sicher ist dies eine mögliche Folge der häufigen Sinusitis. In der Population Baunach findet sich eine Otitis media mit einer Erkrankungshäufigkeit von über 70 Prozent bei Männern und Frauen. Da eine Mittelohrentzündung im wesentlichen als aufsteigender Infekt aus dem Nasen-Rachen-Raum auftritt, ist diese Krankheitshäufung nicht unerwartet. Auch bei anderen paläopathologisch untersuchten Populationen aus dem Gebiet Niederbayerns wurden ähnlich hohe Erkrankungshäufigkeiten gefunden (SCHLOMM 2000 mit 57 Prozent, GRESKY 2006 mit 84 Prozent). Der häufigste Infektionsweg einer Otitis media ist die tubogene Keimaszendierung aus dem Nasen- und Rachenraum. Infekte in diesem Bereich, wie zum Beispiel Sinusitiden oder Rhinitiden, sind also die Hauptursache für die eventuelle Folgeerkrankung des Mittelohrs. Bei der Gegenüberstellung von erkrankten Mittelohren mit ihren über die Tube korrespondierenden Nasennebenhöhlen erwartet man eine deutliche Häufung von Paaren, die in beiden Abschnitten entweder krank oder gesund sind. Diese Erwartungen werden bei der paläopathologischen Untersuchung für die Population Baunach voll bestätigt. Ein statistisch fassbarer Zusammenhang der Pathogenese zwischen der Otitis media und Sinusitis kann mit den Mittelohren aus Baunach aufgezeigt werden. Auch bei der als Komplikation einer Otitis media auftretenden Mastoiditis ist pathophysiologisch eine ähnliche Häufung gleicher Befundqualitäten beider Strukturen logisch. Dies wird in Baunach bei knapp zwei Dritteln der untersuchten Paare ebenfalls bestätigt. Viele der unter- 
suchten Individuen zeigen einen chronische Infektion in intravital mit Schleimhaut ausgekleideten Kompartimenten des Respirationstraktes im Bereich des Craniums (Nasennebenhöhlen). Aber auch mit knöchernen Einschmelzungen einhergehende eitrige Entzündungen sind nachzuweisen. In mehreren Fällen können der Übergang in chronisch eitrige Infektionen durch Fistelbildungen nachgewiesen werden. In der Population Baunach ist eine eitrige Mastoidititis mit intravitaler Abszessperforation an der Mastoidspitze bei mindestens einem Individuum nachweisbar (Bild 19 und 20). Eine ausgeprägte chronische Fistel zwischen Sinus maxillaris und Mundhöhle kann ebenfalls bei mindestens einer Person nachgewiesen werden (Bild 9 bis 12).

Mögliche Ursachen für die große Zahl von verschieden lokalisierten Infektionskrankheiten im Bereich des Gesichtsschädels sind eine starke Erregervirulenz, kreisende Infektionsketten oder auch eine gewisse Immuninsuffizienz, für welche die Ursachen wiederum vielfältig sind. Auch schlechte hygienische Verhältnisse haben sich sicher negativ auf die Erkrankungshäufigkeit und Krankheitsverläufe ausgewirkt. Chirurgische Therapieansätze finden sich bei den Baunacher Individuen nicht. Nur eine ungelenkte Perforation entlang anatomischer Strukturen konnte eine ausgeprägte eitrige Entzündung im Bereich des Gesichtsschädels entlasten. Eine Abszessperforation im Gesichtsschädel kann extracraniell und endocraniell zur fistelnden Drainage führen. Für den Fall der endocraniellen Abszessentlastung sind vielfältige Komplikationen möglich. Ein letaler Ausgang für diese Verläufe ist annehmen.

In der Baunacher Population lassen sich im endocraniellen Raum auch häufig auftretende charakteristische Veränderungen nachweisen. Diese sind zumeist unspezifisch und können in entzündlich oder hämorrhagisch bedingte Läsionen unterschieden werden.

Eine hämorrhagisch verursachter pathologischer Prozess wird in der Regel durch vermehrte Impressionen von Gefäßen gekennzeichnet. Zudem zeigt die knöcherne Oberfläche eine über die Norm ausgebildete poröse Beschaffenheit (SCHULTZ 2001). Zeichen eines chronischen Prozesses beziehungsweise eines in Abheilung 
begriffenen Prozesses sind plattenartige Neubildungen (SCHULTZ 1993). Häufig treten diese Veränderungen in Mischformen auf, wobei ein Ausprägungsmerkmal meist die Läsion dominiert.

Bei den gefundenen endocraniellen Infektionen sind bei knapp vier Fünftel der vorhandenen und untersuchbaren Laminae internae des endocraniellen Raums die Zeichen einer Leptomeningitis tuberculosa festzustellen. Es zeigen von 36 untersuchten Personen 26 die Zeichen einer tuberkulösen Meningitis. Somit ergibt sich ein denkbares Maximum von 72 Prozent. Dies ist eine erschreckend große Häufigkeit einer heute in Deutschland schon beinahe als ausgerottet geltenden Krankheit. Aber man kann nach den vorliegenden Ergebnissen davon ausgehen, dass in der Zeit von 750-900 n. Chr. eine fast vollständige Durchseuchung der Baunacher Bevölkerung mit dem TBC auslösenden Keim Mycobacterium tuberculosis bestanden hat. Da die Infektion nicht zwangsläufig zur manifesten Erkrankung führt, kommen solch große Häufigkeiten wie in Baunach zu Stande. Aber auch in anderen anatomisch-pathologischen Untersuchungen finden sich ähnliche Ergebnisse. So haben laut ULRICI (1944) nach Sektionsergebnissen von Naegeli, Burckhardt und Aschoff Ende des 19. Jahrhunderts und zu Beginn des 20. Jahrhunderts etwa 90-100 Prozent der Erwachsenen anatomische Merkmale früherer Tuberkuloseinfektionen. Aus diesen Untersuchungen weiß man von einer Durchseuchung von ca. 80 Prozent im Alter von 18-21 Jahren. Bis zum 25. Lebensjahr war dann von einer Durchseuchung von nahezu 100 Prozent auszugehen, wobei die Mehrzahl der Infektionen nach ULRICI (1944) nicht über das primäre Stadium der Tuberkulose hinaus reichte. In Baunach ist dies mit dem Auftreten einer meningealen Tuberkulose jedoch geschehen. Es ist davon auszugehen, dass die Tuberkulose in Baunach und anderen Populationen in allen Gesellschaftsschichten und Altersklassen vor Impfmöglichkeiten oder antibiotischer Therapieoption offenbar häufig anzutreffen war. Bei sicher nachgewiesenen durch Eutertuberkulose des Rindes hervorgerufenen humanen TBC-Erkrankungen sowie der möglichen TBC-Infektion von Haustieren (Rinder, Schweine, Ziegen, Kaninchen) durch infizierte Tierhalter (STEFFENHAGEN 1908) ist eine Erkrankung der Nutztiere in der dörflichen Population Baunach sehr wahrscheinlich. Da in diesen Fällen ein häufig langjährig blander Verlauf beschrieben worden ist, kann man dies als wei- 
teren Hinweis auf Nutztier-assoziierte TBC-Infektionen werten. Nach ULRICI (1944) ist auch bemerkenswert, dass die bovinen Typen der TBC-Erreger beim Menschen häufiger zu extrapulmonal auftretenden Tuberkulosen führen.

Die Tuberkelgranulome können bei suffizientem Immunsystem über viele Jahre inaktiv ruhen. Die akute Erkrankung muss also möglicherweise nie ausbrechen. Eine so große Zahl von Tuberkuloseerkrankten kann also auch ein Hinweis auf eine suffiziente Immunantwort der mit TBC infizierten Baunacher Individuen sein. In diesem Fall kann man dann nicht von Tuberkuloseerkrankten, sondern nur von TBC-infizierten Personen sprechen. Diese gesund erscheinenden Personen können jedoch bei schlechter Abwehrlage ohne erneuten Erregerkontakt schnell an akuter TBC erkranken. Auf jeden Fall ist von einer hohen Inzidenz von Tuberkulose in der Population von Baunach auszugehen.

Auch die Cribra orbitalia war in Baunach und anderen paläopathologischen Untersuchungen in unterschiedlichen Häufungen weit verbreitet. So beschreibt DETKEN (1991) in der von ihm untersuchten Population von Barbing eine Häufigkeit der Cribra orbitlia von 24 Prozent. GRESKY (2006) findet in der bajuwarischen Population Harting eine Häufigkeit von 96 Prozent. In Baunach ist eine Cribra orbitalia bei $\mathrm{n}=31 / 37 ; 84$ Prozent festzustellen. Die rein anämisch imponierende Cribra findet sich in Baunach nicht. Es finden sich histologisch Mischformen von entzündlichen und anämisch bedingter Cribra. Eine entzündungsfreie anämische Cribra orbitalia, wie sie häufig bei Untersuchungen großer vorchristlicher und präkolumbischer Siedlungen mit geschichtlich dokumentierten Krieg- und Hungerzeiten gefunden worden ist (SCHULTZ et al. 2001), liegt in Baunach nicht vor. Auch Rachitis oder Skorbut als weitere mögliche Ursache einer Cribra orbitalia lassen sich mikroskopisch nicht nachweisen. Die Genese dieser morphologischen Auffälligkeit der Cribra war bis vor Kurzem nach gängiger Meinung durch Eisenmangel-Anämie bedingt (EL-NAJJAR et al., 1976; WAPLER 2004). Neuere Untersuchungen gehen dagegen von anderen Voraussetzungen aus und favorisieren eine Anämie durch Vitamin $B_{12}$-Mangel (WALKER 2009). Dies muss jedoch aus den in Kapitel 3.10.3 geschilderten Gründen abgelehnt werden, da auch in der modernen Gesellschaft häufig ein Vitamin $\mathrm{B}_{12}$-Mangel vorliegt, im aktuellen Sektionsgut jedoch eine Cribra sehr selten auffällt. Da die Ursachen der Anämie viel- 
fältig sind, ist eine differenzierte und auch fokussierte Diagnostik des knöchernen Materials wichtig (SCHULTZ 1993, 2001). Häufig finden sich auch in direkter Nachbarschaft der Orbita entzündliche Herde, welche sicher auch eine periostale Reizung im Bereich der Orbita begünstigten. Da Entzündungen mit einer Hyperämie des Gewebes einhergehen und Cribra auch als vermutlichen Übergang der periorbitalen Venen zur Diploë angesehen werden (LANG 1979), ist eine Entzündung in der Orbita begünstigend für diese morphologische Auffälligkeit. Dies ist nur durch licht- und rasterelektronenmikroskopische Diagnostik weiter zu differenzieren.

Man kann jedoch sicher sagen, dass eine Cribra orbitalia einen Stressmarker bei schweren Infektionen darstellt. In der Population Baunach sind alle Orbitadächer mit eingeschmolzenen Gruben an Schädeln zu finden, die Zeichen einer endocraniellen TBC aufweisen. Die Beteiligung der Orbita und der Konjunktiven ist bei der Tuberkulose eine häufige Manifestation und Komplikation (FUCHS 1910). Die eingeschmolzenen Gruben können somit als orbitale Manifestation einer TBC verstanden werden. Somit wird in Baunach die entzündliche Komponente der Entstehung einer Cribra orbitalia nach WAPLER (2004) sehr häufig gewesen sein.

Da nur durch rasterelektronenmikroskopische und histologische Untersuchungen zwischen den möglichen Ursachen einer Cribra orbitalia oder eventueller Pseudopathologie unterschieden werden kann, sind verlässliche Studien nur durch eine gesteigerte Zahl an lichtmikroskopischen Untersuchungen von Knochendünnschliffen der Orbita (auch aus vergleichbarem Sektionsgut) zu erreichen. Aktuelle klinische und histopathologische Untersuchungen mit dokumentierten Krankheitsgeschichten können nicht nur bei der Cribra orbitalia den Kausalitätszusammenhang klären und somit die Aussagekraft paläopathologischer Untersuchungen steigern. 


\section{Ausblick}

Die Ergebnisse der Erkrankungshäufigkeiten in der karolingischen Population von Baunach lassen vermuten, dass eine "Bagatellerkrankung" der heutigen Zeit der "Normalzustand" in der Vergangenheit gewesen sein könnte. Die Relationen dieser Begrifflichkeit über die Jahrhunderte kann also auch durch die Paläopathologie beschrieben werden. Anhand der vorliegenden Skelete mit vielfältigen entzündlichen Befunden muss man von einer starken Häufung der bakteriell bedingten Infektionskrankheiten ausgehen. Im Bereich des Gesichtschädels finden sich überwiegend unspezifische Entzündungen im Bereich der Nasennebenhöhlen und somit des oberen Respirationstraktes. Eine Vielzahl an möglichen auslösenden und begünstigenden Faktoren ist denkbar.

Sehr häufig werden bei Individuen mit ausgeprägter Sinusitis auch stark ausgeprägte entzündliche Veränderungen im Bereich des Zahnhalteapparates sowie Karies gefunden. Da postmortal eine Differenzierung zwischen primär bestehenden oder sekundär erfolgten Infektionen in der Regel nicht möglich ist, kann hier nur die enge räumliche Nähe als begünstigender Faktor angeführt werden.

Die Menschen des Gräberfeldes Baunach zeigen aber nicht nur Krankheiten, die auf unspezifische Entzündungen zurückzuführen sind. Überraschend häufig sind auch spezifische Entzündungsherde im endocraniellen Raum zu finden. Man kann von einer ausgeprägten Durchseuchung der Bevölkerung mit Mycobakterium tuberculosis ausgehen.

In der heutigen Zeit ist die tuberkulöse Meningitis in Deutschland eher als selten einzustufen, wenn auch wieder mit leicht zunehmender Tendenz (ROBERTS, BUIKSTRA 2003). Anhand der Ergebnisse des Gräberfeldes Baunach sowie vieler vergleichbarer paläopathologischer Untersuchungen (KREUTZ 1997, SCHLOMM 2000, GRESKY 2006, ROUMELIS 2007) muss man allerdings von einer vollkommen anderen Situation in der Vergangenheit ausgehen. Alle genannten Autoren beschreiben eine endocranielle TBC-Häufigkeit zwischen zwölf (Population von Straubing, KREUTZ 1997) und 45 Prozent (Population von Barbing/Kreuzhof, SCHLOMM 2000). Es ist anzunehmen, dass diese chronische 
Infektionskrankheit mit möglichen langjährigen inaktivierten Erregern eine Erkrankung war, mit der jedes Individuum Kontakt hatte. Eine Durchseuchung der Bevölkerung muss somit angenommen werden. Ob diese Durchseuchung zeitlich und örtlich begrenzt verlief kann nur durch vergleichende Untersuchungen sicher belegt werden. In der karolingischen Zeit mit dünn besiedelten und ländlich geprägten Gebieten wurde bisher eine Vorherrschaft der extrapulmonalen Tuberkulosemanifestationen angenommen (REDECKER 1958). Dabei ist die Hauttuberkulose unter dem Sammelbegriff des „Aussatzes“ oder „Skrophulae“ am wahrscheinlichsten. Anhand der vorliegenden Ergebnisse aus Baunach wurden nun jedoch auch endocranielle Erregerherde für die Karolingerzeit nachgewiesen.

Noch in der Mitte des 20. Jahrhunderts stellte die Tuberkulose in Mitteleuropa eine weit verbreitete Krankheit dar. Die schlechten Lebensbedingungen in der von Mangel und Vertreibung geprägten Nachkriegszeit begünstigten diesen Zustand.

Nur eine strikte Prävention, Meldepflicht und Massenscreening (Röntgenzüge bzw. -busse für Thoraxaufnahmen) halfen, diesen Zustand mit Hilfe moderner Antibiotikatherapie einzudämmen. Auch in der Tierhaltung fand sich der TBCErreger und konnte nur durch intensive Prävention mittels Impfung und strengen Hygienerichtlinien sowie gesetzlich festgeschriebener Fleischbeschau eingedämmt werden. Diese Strategie brachte den Erfolg, dass man Ende des 20. Jahrhunderts die Krankheit in Deutschland fast als besiegt betrachtete.

Die paläopathologischen Ergebnisse legen nahe, dass diese Bakterienart und die von Ihr verursachte Infektion den Menschen sowie seine Haustiere seit Tausenden von Jahren begleitet haben (LÖFFLER 1958, HERSHKOVITZ et al. 2008). Im Fall des Gräberfeldes Baunach muss man von vielen infizierten Individuen ausgehen. Da allerdings sehr viele Skelete auch im fortgeschrittenen Lebensalter Zeichen einer chronischen beziehungsweise akut exazerbierten TBC-Infektion zeigen, ist eine relativ suffiziente Immunreaktion auf diese Erkrankung wahrscheinlich. Ein großer Anteil der Bevölkerung lebte viele Jahre lang nach der Erstinfektion mit möglicherweise durch Haustiere übertragene Mykobakterien völlig symptomfrei (WEBER 1907). Untersuchungen aus der Ära nach dem Bekanntwerden der durch Mykobakterien verursachten Tuberkulose beschreiben das große Spek- 
trum der durch Haustiere infizierten Tuberkulosepatienten sowie deren klinischer Verlauf. Klinische Untersuchungen aus der Hochphase der Tuberkulose um 1900 belegen eine Vielzahl an durch Nutztierkontakt bedingten Tuberkuloseerkrankungen. Diese belegen aber auch, dass sich die humanen und die tierischen Tuberkuloseformen („Perlsucht“) morphologisch, kulturell sowie in Ihrem pathogenen Verhalten unterscheiden (KOSSEL 1905). Der klinische Verlauf gestaltete sich nicht zwangsläufig fulminant nach Infektionen mit dem bovinen Tuberkuloseerreger.

Ein vom heutigen Verlauf abweichendes Krankheitsbild mit gemäßigterem Verlauf ist bei der großen Häufung von TBC-Zeichen bei fast drei Vierteln der Individuen aus Baunach (26/36) möglich. Theoretisch denkbar, aber wohl nicht wahrscheinlich, ist aber auch eine Siedlung für TBC-Kranke, also "Aussätzige“.

Das Immunsystem eines Erwachsenen im Frühmittelalter musste eine bakterielle Entzündung zwangsläufig beherrschen, da er ansonsten ohne medikamentöse antibiotische Unterstützung nicht das adulte Lebensalter erreicht hätte. Dies deutet wieder auf einen überwiegend suffizienten Ernährungszustand sowie auf mögliche Behandlung der bakteriell bedingten Erkrankungen zu Lebzeiten hin. Antiinfektiöse Wirkstoffe sind in der Naturheilkunde schon lange bekannt (vgl. Lehrbücher Pharmazie; z. Bsp. IRION 1942). Ob und welche Substanzen zum Einsatz kamen, ist mit Hilfe der benutzten Methoden nicht zu klären. Sicher können weitere archäologische und historische Untersuchungen vergleichbarer Populationen in dieser Frage weiter helfen, wenn auch eine begleitende paläopathologische Untersuchung sowie enge interdisziplinäre Zusammenarbeit bei der Auswertung und Beurteilung der Ergebnisse erfolgen.

Da sich in dem vorliegenden Material sehr viele entzündliche Spuren am Schädel finden, ist sicher auch das Postcranium von entzündlichen Veränderungen betroffen. Spuren chronisch entzündlicher Veränderungen an den Langknochen sind zu erwarten. 
Es ist davon auszugehen, dass Entzündungen mit konsekutiver Sepsis bei fulminantem Verlauf und Bakteriämie eine der häufigsten Todesursachen für den Menschen in der frühmittelalterlichen Population Baunach waren. Sichere Spuren maligner tumoröser Veränderungen als eine der häufigsten Todesursachen in der heutigen Zeit wurden am Schädel der Baunacher Individuen nicht nachgewiesen.

Die Differenzierung auffälliger Läsionen in intravital entstandene Spuren pathologischer Prozesse und postmortal diagenetisch bedingter Läsionen ist für die genaue Befundung wesentlich. Bei vielfältig möglichen postmortalen Beschädigungsmöglichkeiten (SCHULTZ 1997) ist dafür die Untersuchung im Rasterelektronenmikroskop sowie Lichtmikroskop notwendig. 


\section{Zusammenfassung}

Aus der frühmittelalterlichen Population Baunach wurden 52 Schädel in dieser Untersuchung bearbeitet werden. Es liegt ein deutlicher Männerüberschuss vor (Männer 56 Prozent, Frauen 29 Prozent, nicht bestimmbare Individuen 15 Prozent). Bei der paläopathologischen Bearbeitung wurden makroskopische, endoskopische sowie licht- und rasterelektronenmikroskopische Untersuchungstechniken herangezogen.

Eine hohe Zahl entzündlicher Veränderungen im Bereich des Gesichts und Hirnschädels kennzeichnen diese Population. Es muss von einer ausgeprägten Häufung chronischer Infektionen der Nasennebenhöhlen ausgegangen werden, da 94 Prozent der Männer und sogar 100 Prozent der Frauen mindestens eine erkrankte Kieferhöhle zeigen. Da eine Entzündung der Nasennebenhöhlen unbehandelt häufig in ein chronisches Stadium übertritt, liegen somit im Bereich des Craniums Infektionsreservoire vor. Die endoskopische Untersuchung der Mittelohren belegt, dass eine Infektion der Nasennebenhöhlen eine Voraussetzung für Mittelohrentzündungen darstellt. In der Population Baunach ist kein Mittelohr erkrankt, wenn die korrespondierenden Nasennebenhöhlen gesund sind. Von den untersuchten Baunacher Individuen zeigen drei Viertel eine Otitis media. Diese gilt ätiologisch als Voraussetzung für die Ausbildung einer Mastoiditis, welche in Baunach ebenfalls mit 80 Prozent bei den Frauen und 52 Prozent bei den Männern zu finden ist. Auch abszedierende Befunde mit Einschmelzungen im Bereich der Mastoidspitze zur Abszessentlastung im Sinne einer heute sehr selten zu findenden Bezold'schen Mastoiditis können in der Population Baunach nachgewiesen werden.

Auch im Endocranialraum lässt sich eine große Häufigkeit von entzündlichen Erkrankungen nachweisen. Eine meningeale Reizung kommt bei Männern mit 80 Prozent und bei Frauen sowie Individuen mit nicht bestimmbarem Geschlecht mit 90 Prozent sehr häufig vor. Aufgrund der morphologischen Merkmale muss der dringende Verdacht auf eine Leptomeningitis tuberculosa bei 85 Prozent der Männer und 73 Prozent der Frauen als Ursache der meningealen Reizungen geäußert 
werden. Somit wäre mit dem denkbaren Maximum von 72 Prozent der Gesamtzahl aller an meningealen Reizungen leidenden Baunacher eine eindrucksvolle Erkrankungshäufigkeit bezüglich einer extrapulmonal gestreuten Tuberkulose festzustellen. Die Möglichkeit einer Siedlung für Kranke oder “Aussätzige“ muss in Betracht gezogen werden, da sich in örtlich vergleichbaren anderen frühmittelalterlichen Populationen eine geringere Häufigkeit an TBC findet.

Orbitadächer, an denen eine Cribra orbitalia nachgewiesen wurde, lassen durch die topographisch nahe Beziehung zum Endocranium eine meningeale Reizung begünstigende Voraussetzung vermuten. Von 32 untersuchten Paaren zeigen 25 Paare, also 78 Prozent, sowohl im Endocranium als auch am Orbitadach pathologische Befunde. Da eine tuberkulös bedingte meningeale Reizung als chronische Erkrankung anzusehen ist, kann die ebenfalls ausgeprägte Häufung einer Cribra orbitalia sowohl als entzündliche Begleitreaktion wie auch als bei chronischer Anämie auftretende kompensatorische Knochenmarkshypertrophie interpretiert werden. Diese Befunde wurden lichtmikroskopisch bestätigt. Eine rein entzündliche Cribra wie auch eine rein anämisch verursachte Cribra finden sich nicht. Es sind jeweils Mischformen in den Präparaten dargestellt. Auch Skorbut oder Rachitis als Ursache einer Cribra wurden nicht nachgewiesen.

Die zahlreichen ausgeprägten Entzündungen mit zum Teil intracraniell gelegenen putriden Fistelbildungen werden ohne entsprechende Behandlung häufig den Tod des Individuums nach sich gezogen haben. Somit sind bei der errechneten durchschnittlichen Lebenserwartung von nur 35,1 Jahren bei Geburt sicher die Infektionskrankheiten als wesentlicher Faktor für einen frühen Tod zu nennen. Da sich bei Männern bei Geburt eine Lebenserwartung von 43,6 Jahren errechnet, Frauen dagegen nur mit einer Lebenserwartung von 33,0 Jahren geboren werden, muss ebenfalls eine große vitale Bedrohung durch Schwangerschaften und die damit verbundenen Komplikationen angenommen werden.

In der Baunacher Population finden sich keine lebensgefährlichen Traumata am Schädel. Entzündliche Veränderungen am äußeren Schädeldach sind jedoch bei Männern mit 68 Prozent $(n=17 / 25)$ recht häufig. Bei männlichen Individuen sind auch Hinweise auf kleinere Wunden am Schädeldach häufig. Diese können eine 
Eintrittspforte für die resultierende Kopfschwartenentzündung dargestellt haben. Auch wenige weibliche $(n=2 / 9)$ und unbestimmbare Individuen $(n=1 / 4)$ zeigen Spuren im Sinne einer Kopfschwartenentzündung. Bei Fehlen von traumatischen Spuren am Schädeldach kann hier jedoch ein Befall mit Parasiten (Pediculus humanus capitis, Kopflaus) als Ursache dieser Erkrankung angenommen werden. Indirekt kann somit bei nichtvorhandenen traumatischen Spuren an weiblichen und kindlichen Schädeln auf ein weitestgehend gewaltfreies dörfliches Sozialleben geschlossen werden. 


\section{Anhang: Bild 1 - 30}

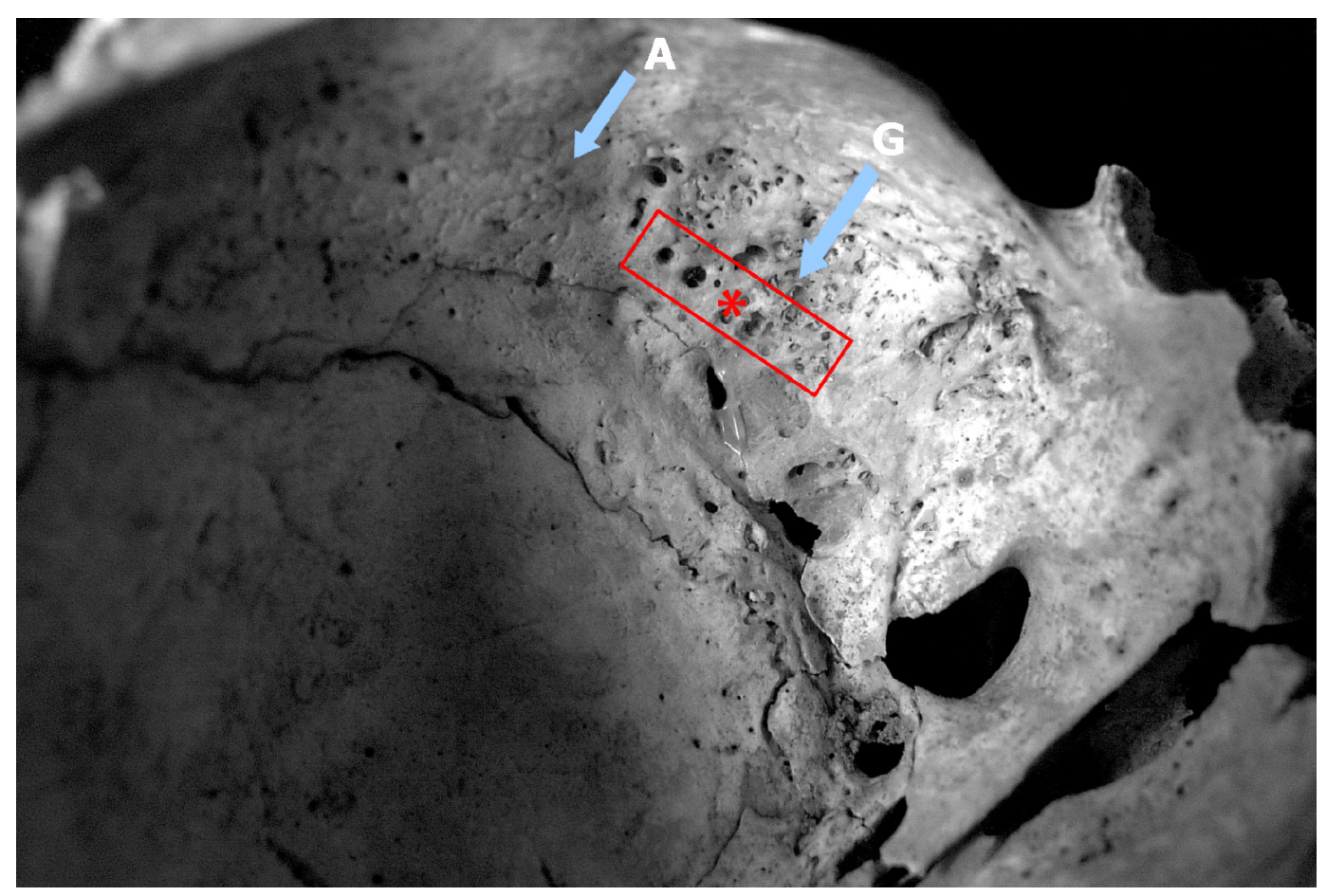

Bild 1: Bau 1, weiblich, 60-65 Jahre, Ala major ossis sphenoidalis links

Pfefferkorngroße konfluierende Grübchen (G), netzartige Gefäßimpressionen in fein porösen Auflagerungen (A).

$\rightarrow$ chronisch entzündlicher Prozess einer meningealen Reizung, Verdacht auf endocranielle TBC

* Areal der Probenentnahme für Histologie, siehe Bild 2 


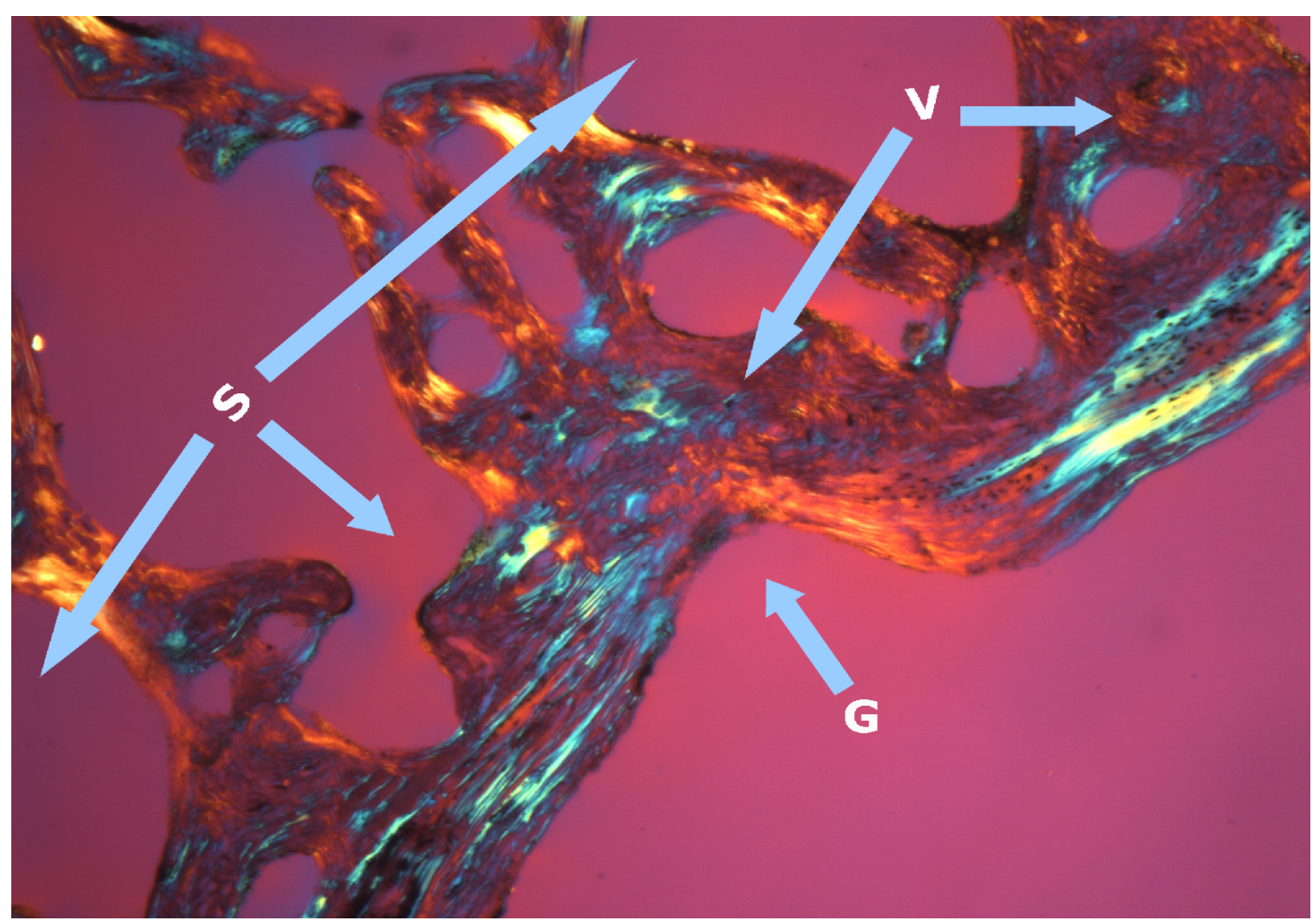

Bild 2: Bau 1, Ala major ossis sphenoidalis links

Betrachtung mit dem Mikroskop im polarisierten Durchlicht unter Verwendung

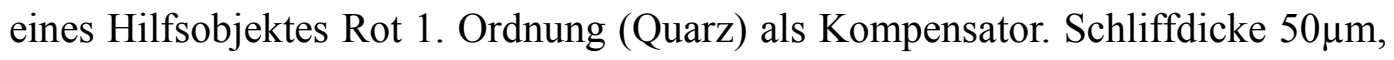
Vergrößerung $25 \mathrm{x}$

Langsam progredienter osteolytischer Prozess mit Howship'schen Lakunen. Wohl nicht hämorrhagisch, eher entzündliche Genese. In Spongiosa regional große Zellen (S) erkennbar, teilweise mit verdickten Knochenbälkchen im Sinne von Vernarbungen (V). Eigentliche Corticalis reduziert, aber sekundär durch reaktive Knochenneubildung scheinbar verdickt und mit Gefäßimpressionsmulden bedeckt.

$\rightarrow$ nicht sehr aggressiver, von Hirnhaut ausgehender Prozess, Verdacht auf TBC 


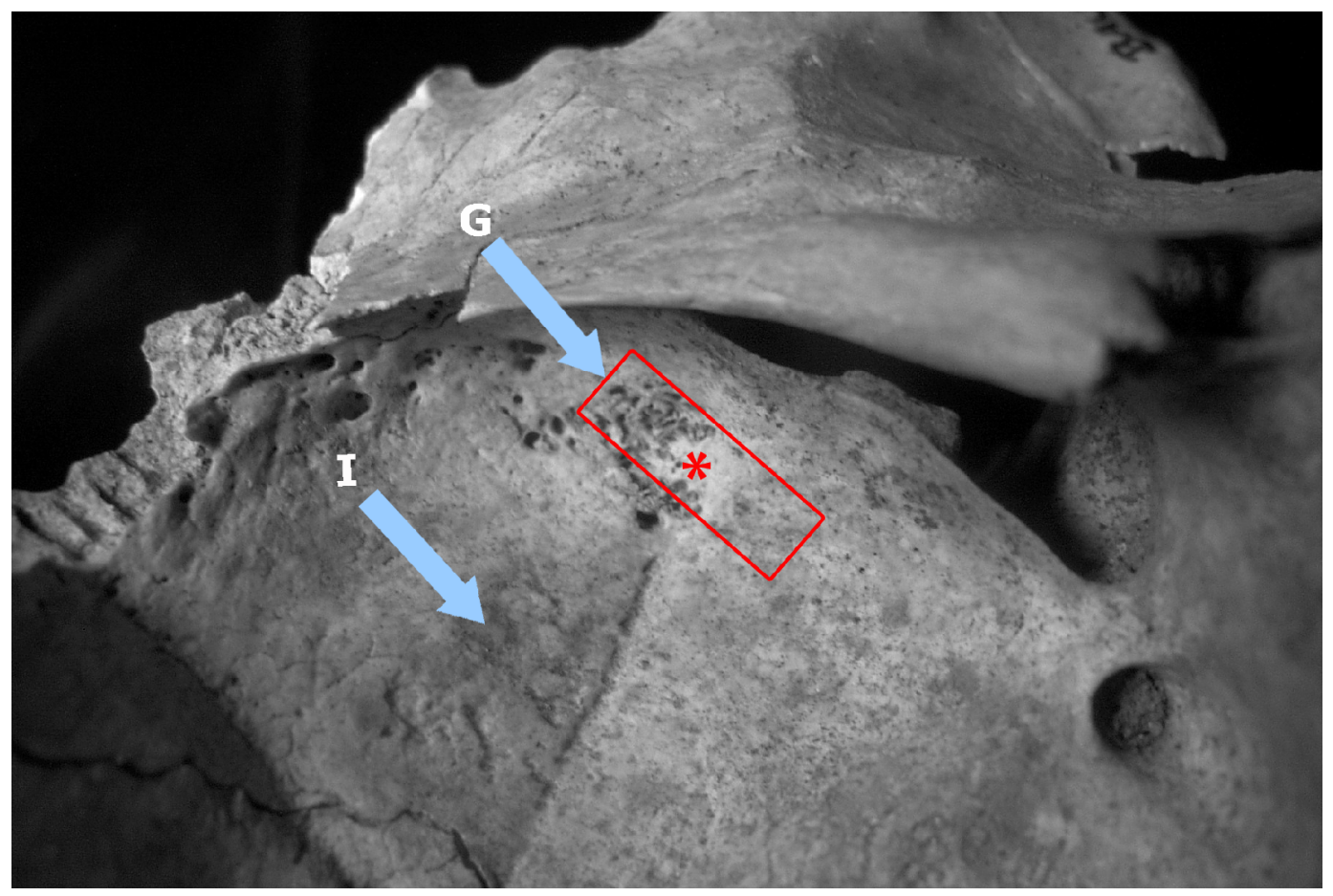

Bild 3: Bau 6, männlich, 35-49 Jahre, Ala major ossis sphenoidalis links

Konfluierende Grübchen (G) mit vermehrt ausgebildeten irregulären Gefäßimpressionen (I) sowie akut entzündliche und partiell lytisch erscheinende Läsion in Bildmitte

$\rightarrow$ chronisch entzündlicher Prozess mit akuter Exacerbation, Verdacht auf endocranielle TBC

* Areal der Probenentnahme für Histologie, siehe Bild 4 


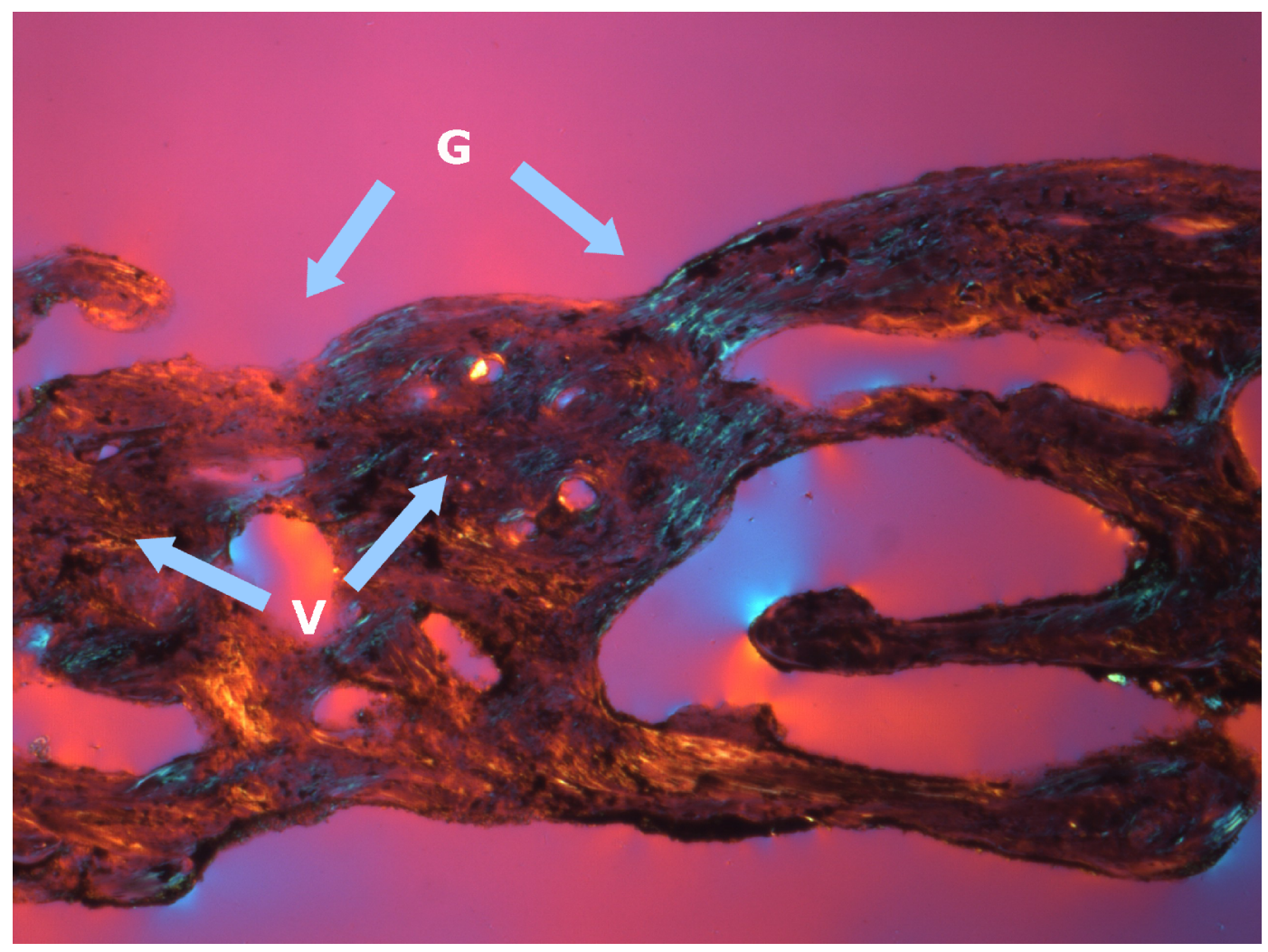

Bild 4: Bau 6, Ala major ossis sphenoidalis links

Betrachtung mit dem Mikroskop im polarisierten Licht unter Verwendung eines Hilfsobjektes Rot 1. Ordnung (Quarz) als Kompensator. Schliffdicke $50 \mu \mathrm{m}$, Vergrößerung $25 \mathrm{x}$

Teilweise reaktiv verdickte Corticalis bei osteolytischem Prozess mit Howship'schen Lakunen. Wenige druckatrophische Grübchen (G). Stellenweise Corticalis nicht vorhanden. In Spongiosa zum Teil größere Areale mit vernarbten und verdickten Spongiosabälkchen (V).

$\rightarrow$ nicht sehr aggressiver, von Hirnhaut ausgehender chronischer Prozess, Verdacht auf endocranielle TBC 


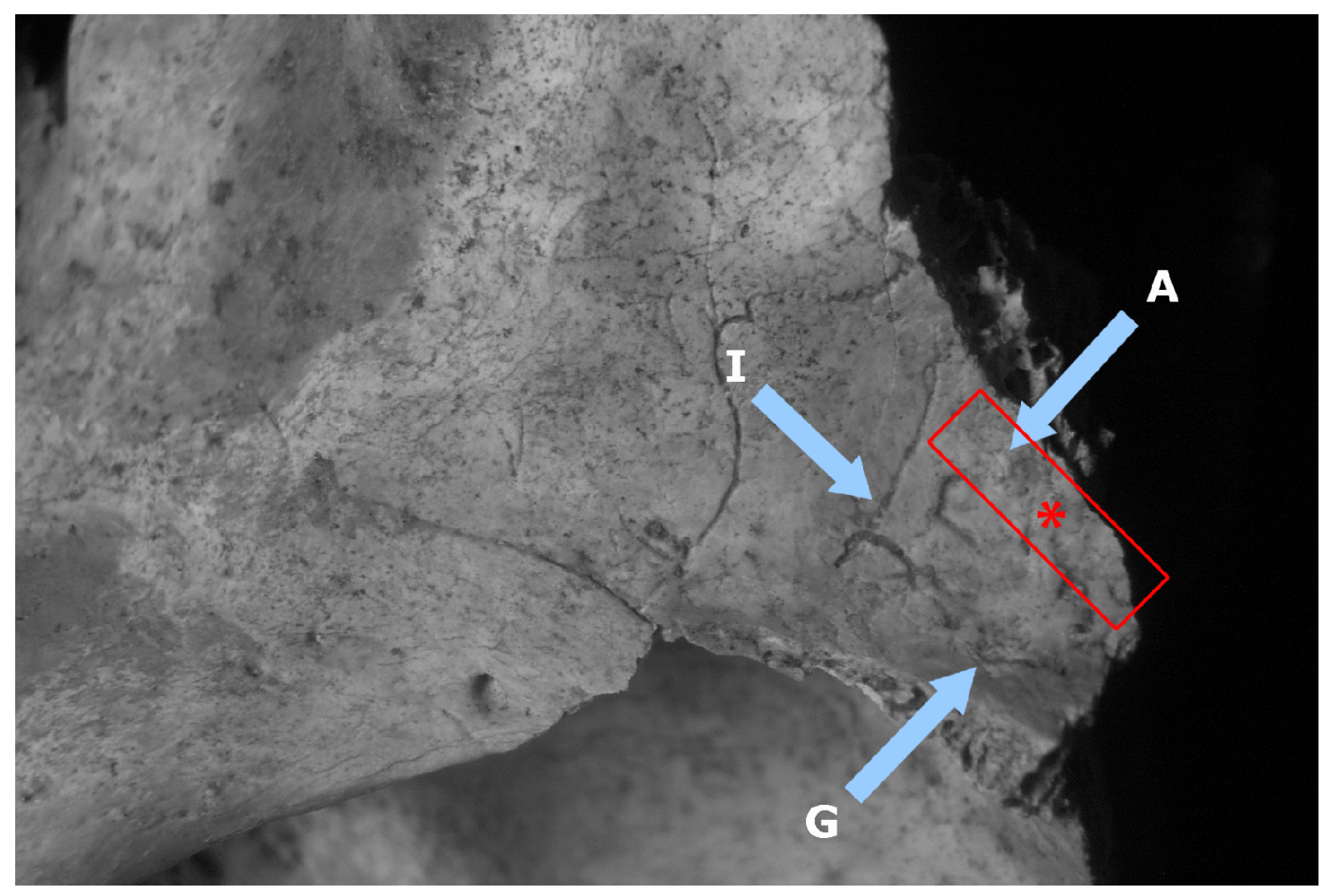

Bild 5: Bau 6, männlich, 35-49 Jahre Os frontale, Fossa cranii anterior links

Konfluierende Grübchen $(G)$ mit glatter Oberfläche und Gefäßimpressionen (I) in Peripherie.

$\rightarrow$ in Abheilung begriffener meningealer Prozess, Verdacht auf druckatrophische Impressionen verursacht durch Tuberkelgranulome

* Areal der Probenentnahme für Histologie, siehe Bild 6 


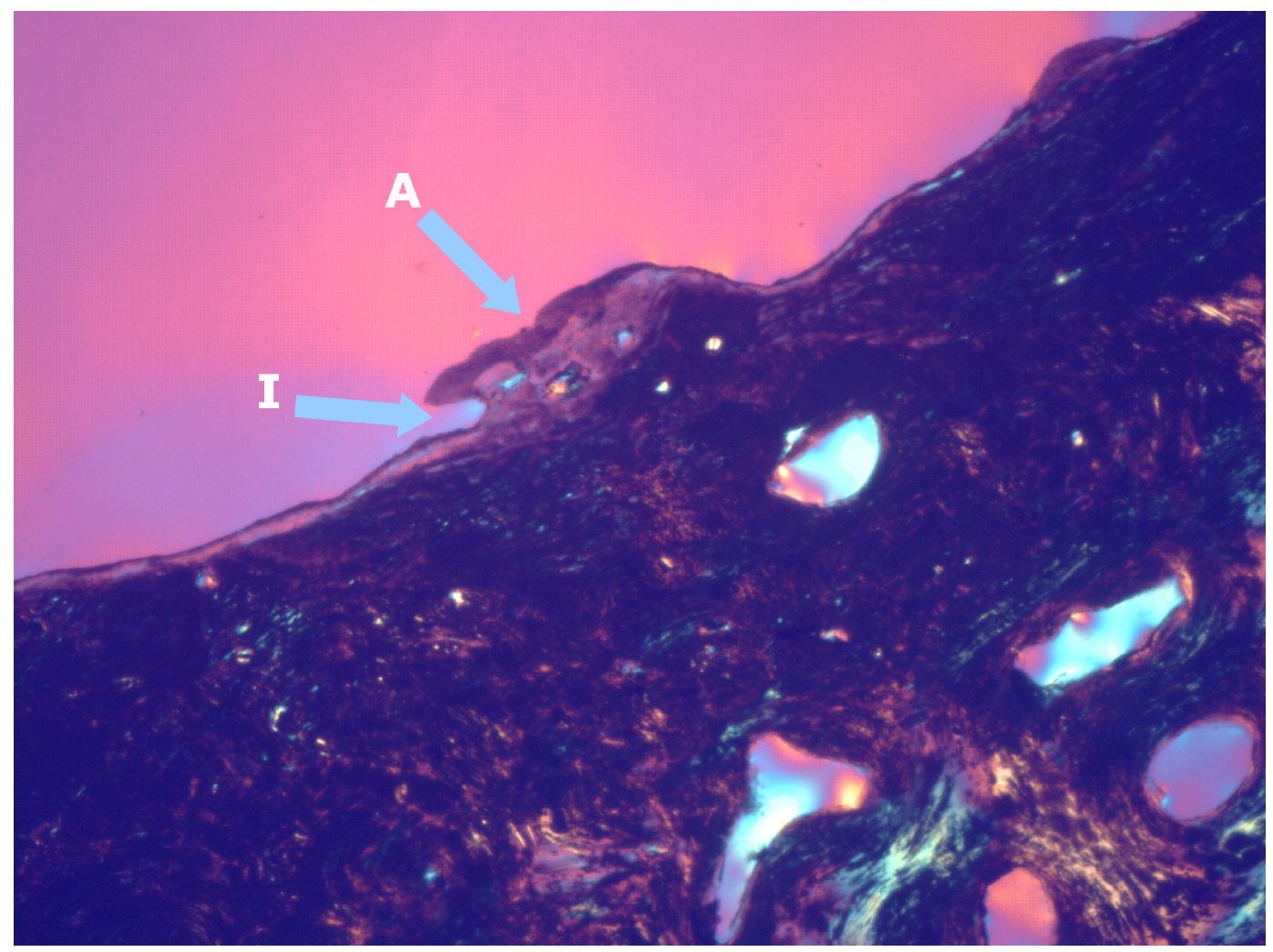

Bild 6: Bau 6, Os frontale, Fossa cranii anterior links

Betrachtung mit dem Mikroskop im polarisierten Durchlicht unter Verwendung eines Hilfsobjektes Rot 1. Ordnung (Quarz) als Kompensator. Schliffdicke 25 $\mu \mathrm{m}$, Vergrößerung $25 \mathrm{x}$

Auf Corticalis sichtbare reaktive Neubildung. Auch Gefäßimpressionen (I) erkennbar. Zum Teil neugebildeter Faserknochen als Oberflächenauflagerungen (A). Keine Auffälligkeiten innerhalb der Spongiosa.

$\rightarrow$ meningeale Reaktion mit Entzündungszeichen 


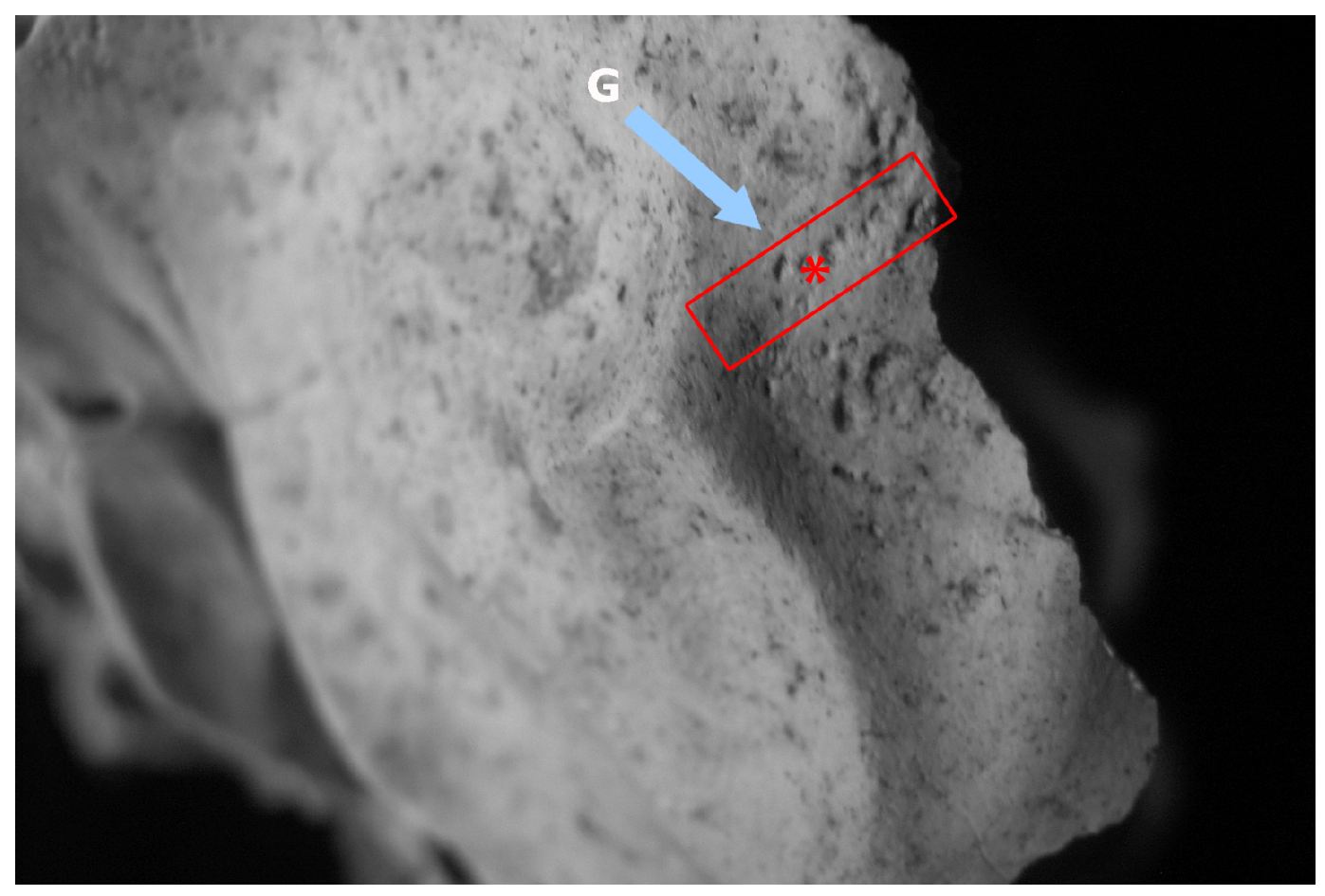

Bild 7: Bau 19, männlich, 50-55 Jahre Os sphenoidale, Fossa cranii anterior rechts

Verstärkte Impressiones digitatae als Zeichen für vermehrten Hindruck. Konfluierende reizlose Grübchen $(\mathrm{G})$ mit fein poröser Peripherie.

$\rightarrow$ chronischer meningealer Prozess, reizlose inaktive druckatrophische Granulomimpressionen

* Areal der Probenentnahme für Histologie, siehe Bild 8 


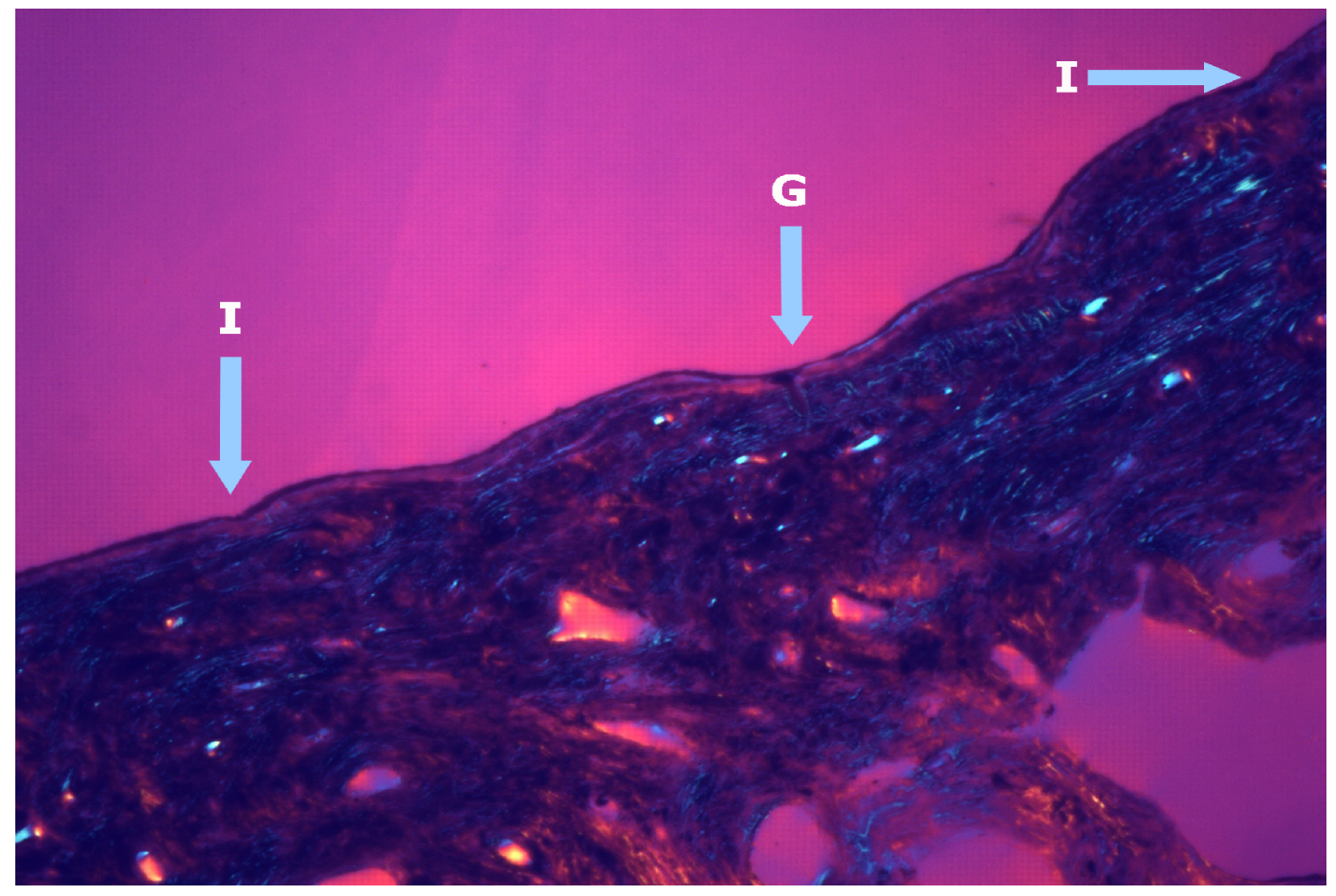

Bild 8: Bau 19, Os sphenoidale, Fossa cranii anterior rechts

Betrachtung mit dem Mikroskop im polarisierten Licht unter Verwendung eines Hilfsobjektes Rot 1. Ordnung (Quarz) als Kompensator. Schliffdicke $25 \mu \mathrm{m}$, Vergrößerung $25 \mathrm{x}$

Auf Corticalis mehrere Gefäßimpressionen (I) und Grübchen (G) mit glatter reizloser Oberfläche sichtbar. Partiell ausgedünnte Corticalis. Keine akuten Entzündungszeichen. Spongiosa regelrecht und ohne Vernarbungen.

$\rightarrow$ chron. Entzündlich-hämorrhagischer Prozess (inaktive Leptomeningitis tuberkulosa mit Tuberkelgranulomimpressionen) 


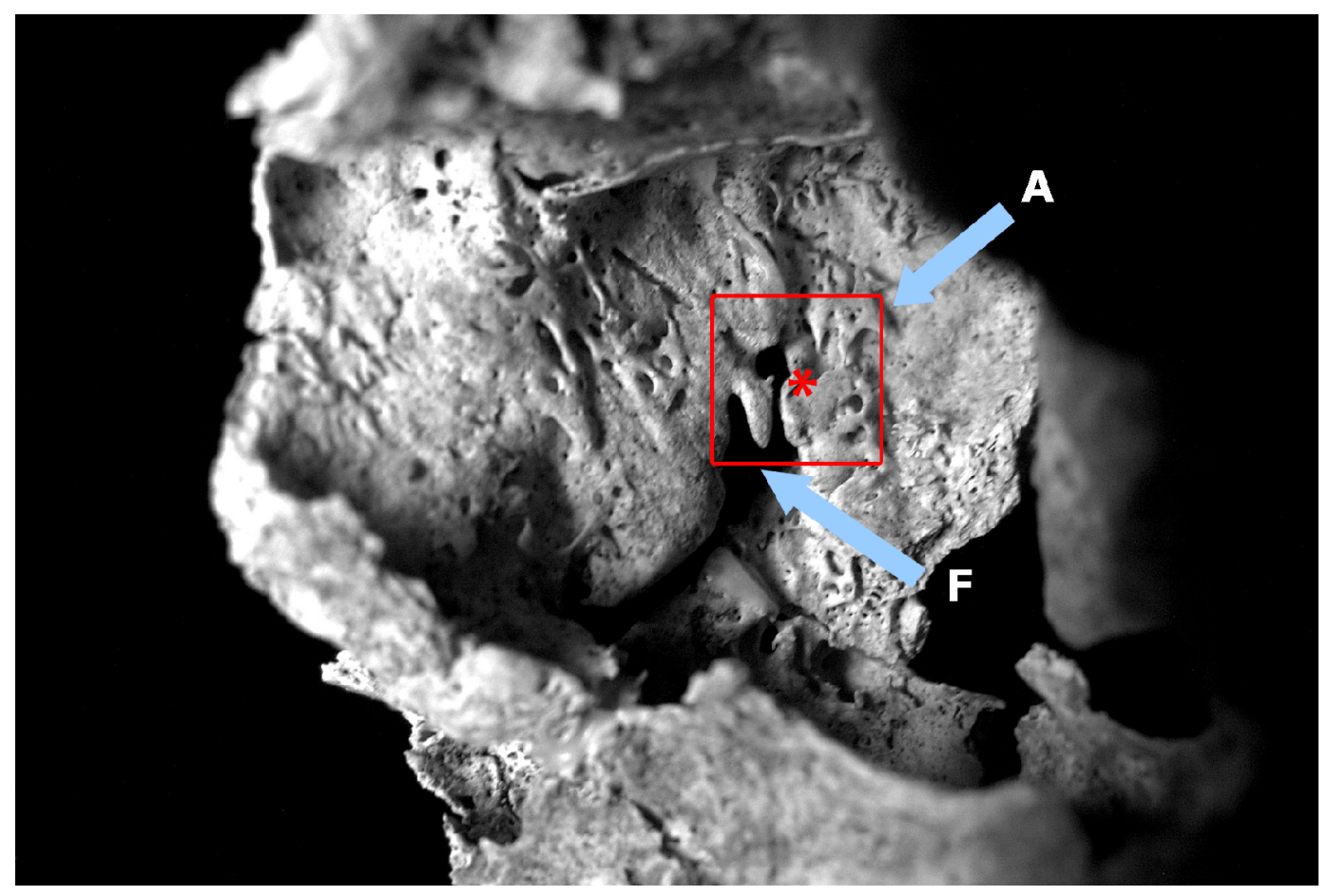

Bild 9: Bau 9, männlich, 60-69 Jahre, Sinus maxillaris links

Sinusboden massiv durch chronisch eitrige Sinusitis maxillaris verändert. Eingeschmolzener Fistelgang (F) zur Mundhöhle. Frische entzündliche Auflagerungen (A) bei chronischer Infektion.

$\rightarrow$ intravital in Mundhöhle perforierender Abszess bei eitriger Sinusitis maxillaris

* Areal für endoskopische Untersuchung, siehe Bild 10 


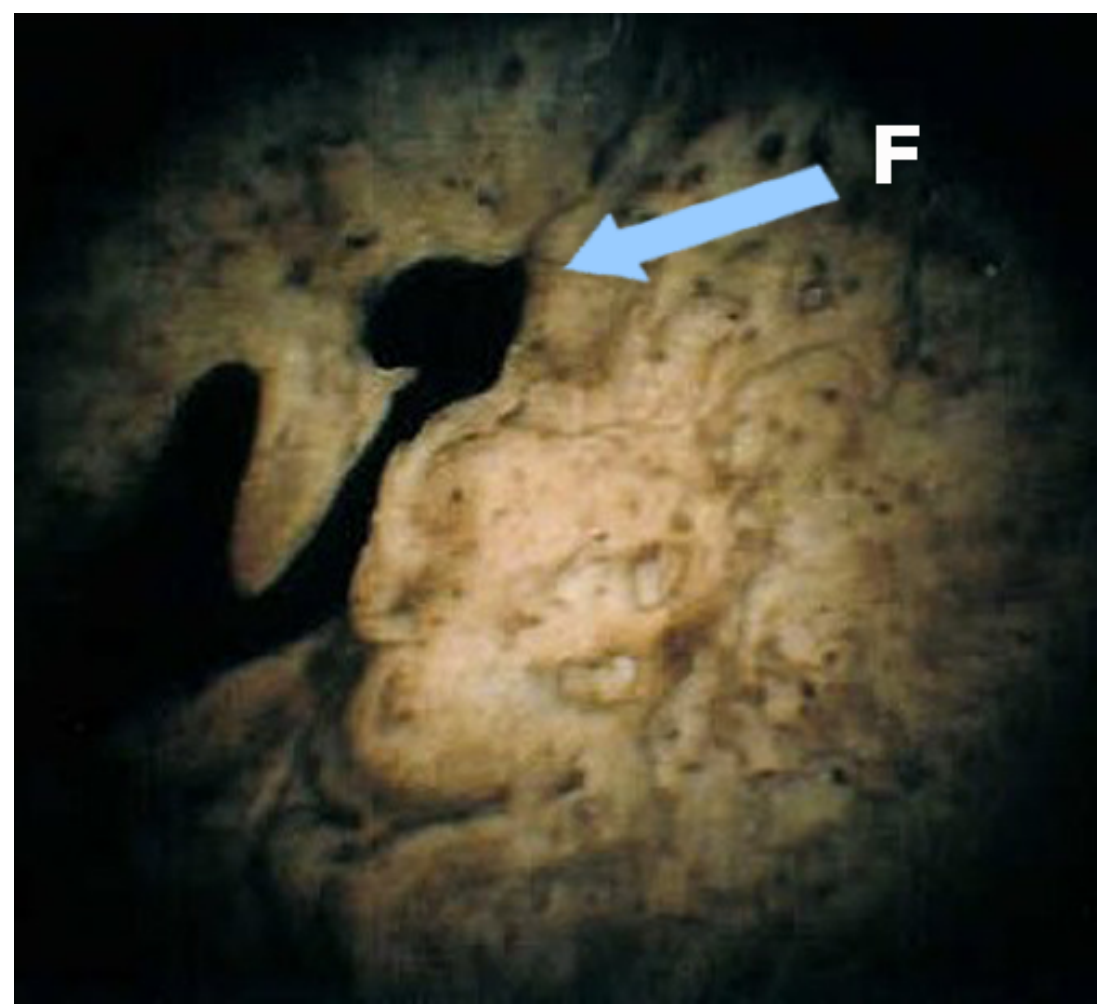

Bild 10: Bau 9, Boden des Sinus maxillaris links (Endoskop)

Boden des Sinus maxillaris mit intravitaler Perforation im Sinne eines Fistelganges (F) zur Mundhöhle. Chronische eitrige Infektion des Sinus maxillaris mit Entlastung des Abszesses in die Mundhöhle. Kein Hinweis auf chirurgische Behandlung des Kieferhöhlenabszesses. 


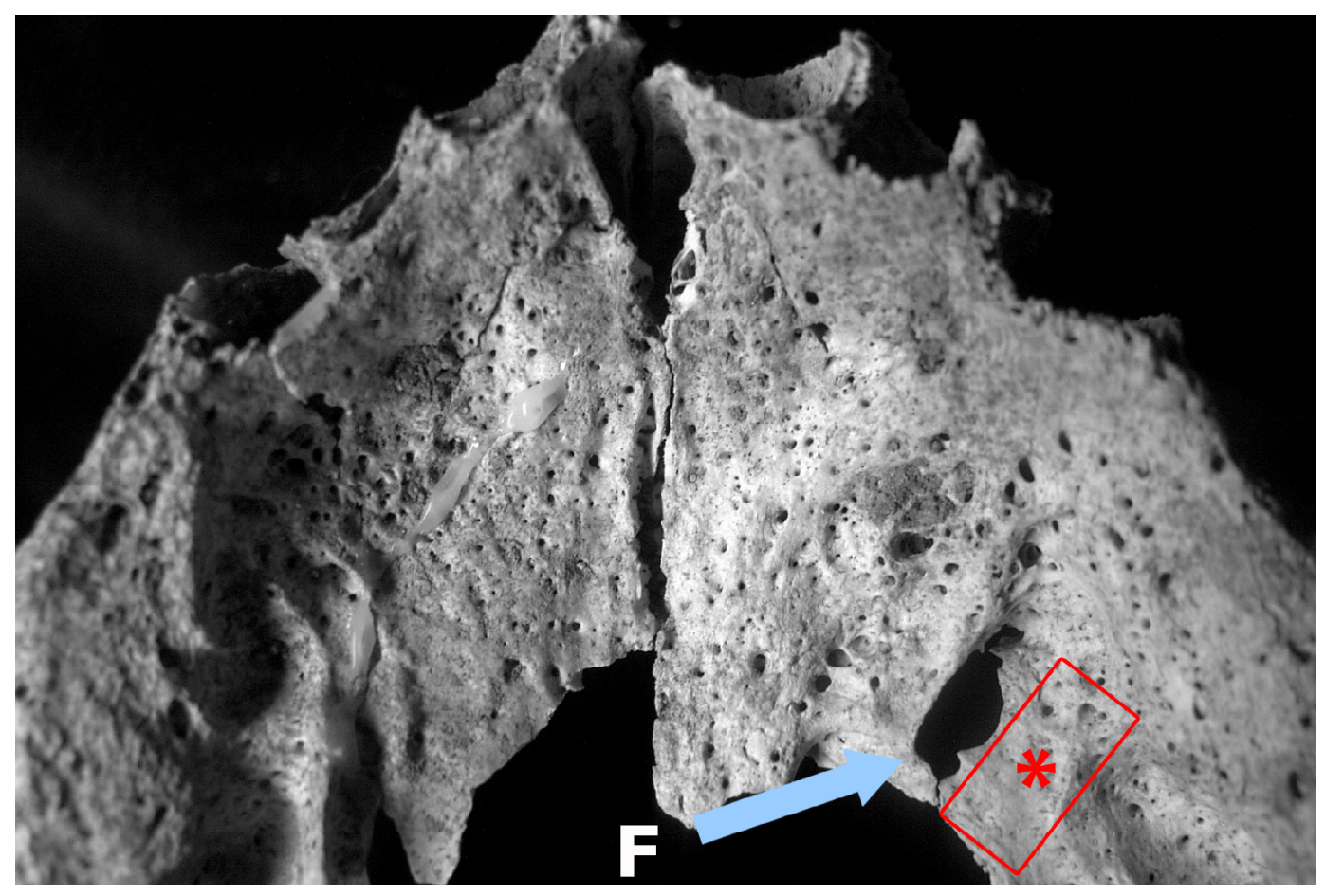

Bild 11: Bau 9, harter Gaumen mit Fistelgang aus Sinus maxillaris links

Durch knöchernes Mundhöhlendach perforierte Sinusitis maxillaris mit Fistelgang (F).

* Areal der Probenentnahme für Histologie, siehe Bild 12 


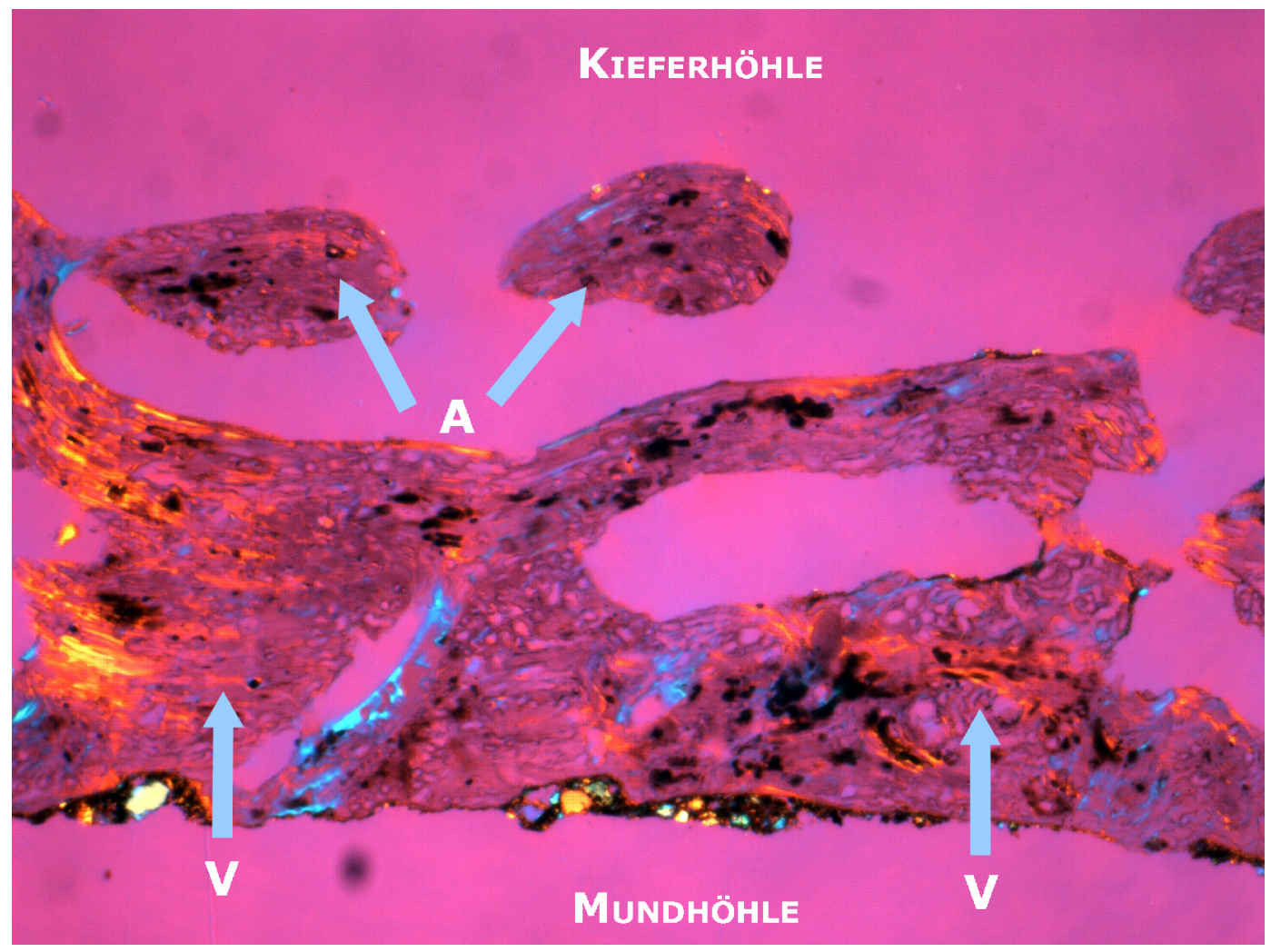

Bild 12: Bau 9 Sinus maxillaris links, harter Gaumen

Betrachtung mit dem Mikroskop im polarisierten Durchlicht unter Verwendung eines Hilfsobjektes Rot 1. Ordnung (Quarz) als Kompensator. Schliffdicke 50 $\mu \mathrm{m}$, Vergrößerung $25 \mathrm{x}$

Struktur von Corticalis und Spongiosa aufgehoben. Ausgeprägte Vernarbungen (V) und Auflagerungen (A) am Boden der Kieferhöhle.

$\rightarrow$ Chronisch entzündlicher Prozess. Putride abszedierte Sinusitis maxillaris. 


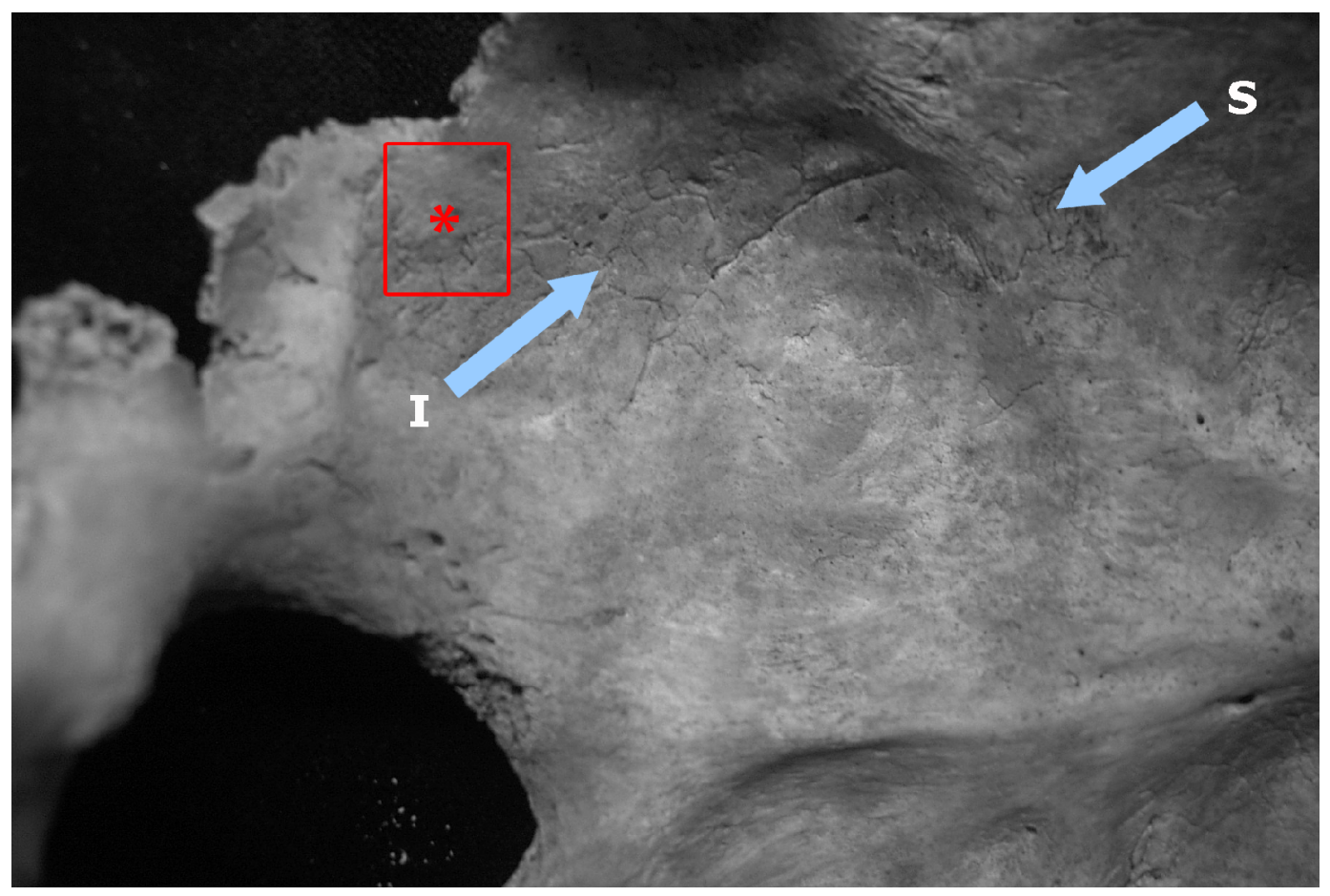

Bild 13: Bau 35-2, Os occipitale, Fossa cranii posterior rechts

Irreguläre netzartige Gefäßimpressionen in plattenartigen Neubildungen, Symptomatik makroskopisch ausgehend vom Rand des Sinus transversus (S). Deutliche Gefäßimpressionen (I) und Impressiones digitatae als Zeichen für verstärkten Hirndruck.

$\rightarrow$ chronische meningeale Reizung und Perisinusitis

* Areal der Probenentnahme für Histologie, siehe Bild 14 


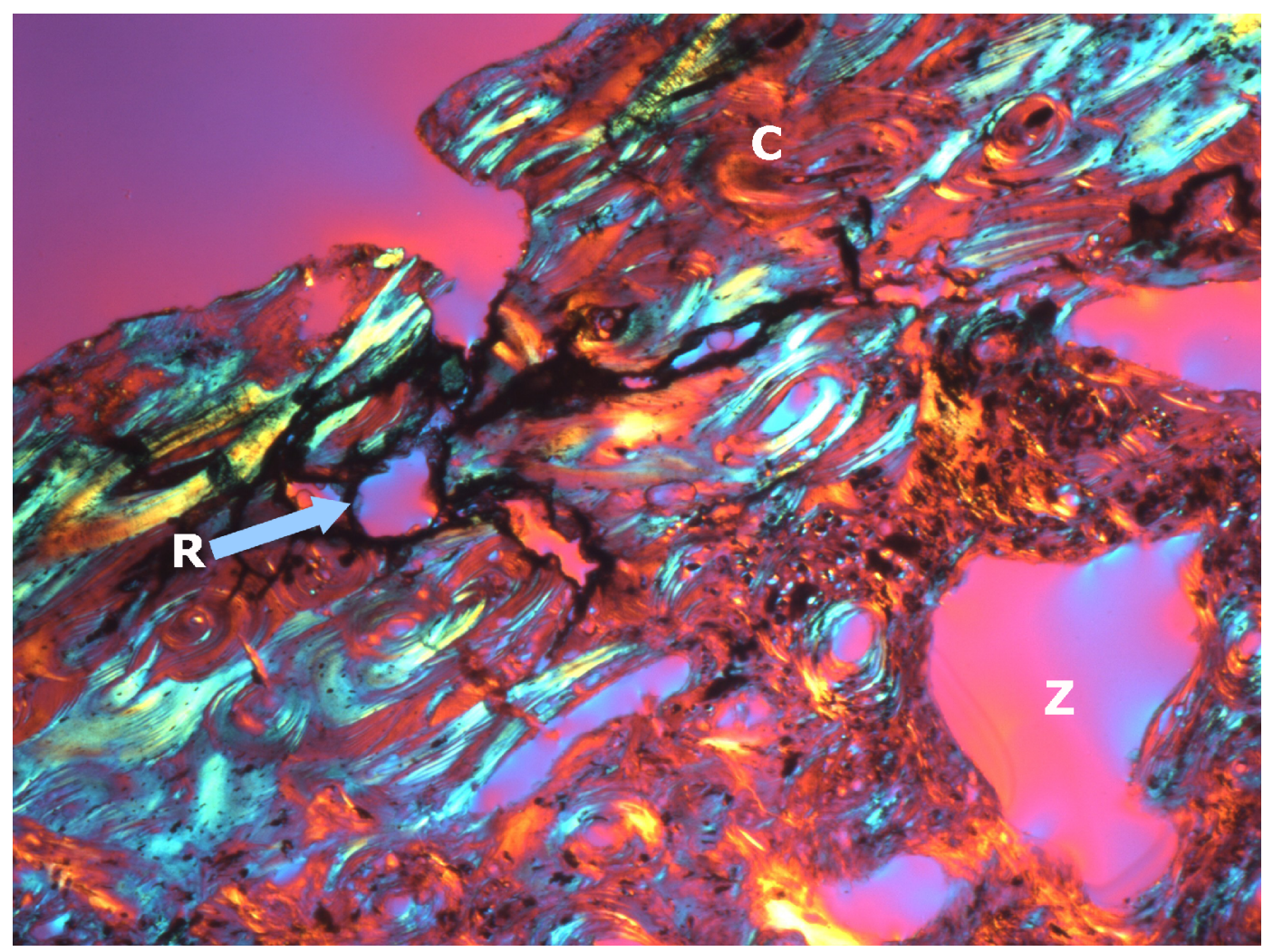

Bild 14: Bau 35-2, Os occipitale, Fossa cranii posterior rechts

Betrachtung mit dem Mikroskop im polarisierten Durchlicht unter Verwendung eines Hilfsobjektes Rot 1. Ordnung (Quarz) als Kompensator. Schliffdicke 25 $\mu$ m, Vergrößerung $25 \mathrm{x}$

Knochen stellenweise stark verdünnt. Markraum mit deutlich vergrößerten Spongiosazellen $(Z)$, diese allerdings zahlenmäßig reduziert (an manchen Stellen verschwunden und dafür sklerosierter Knochen). Narbig verdickte und unregelmäBige Corticalis (C). Plumpe und vernarbte Spongiosabälkchen. Überall Resorptionszonen (R) sichtbar.

$\rightarrow$ chronische meningeale Reizung, partiell abgeheilt und vernarbt aber auch akut entzündliche Areale 


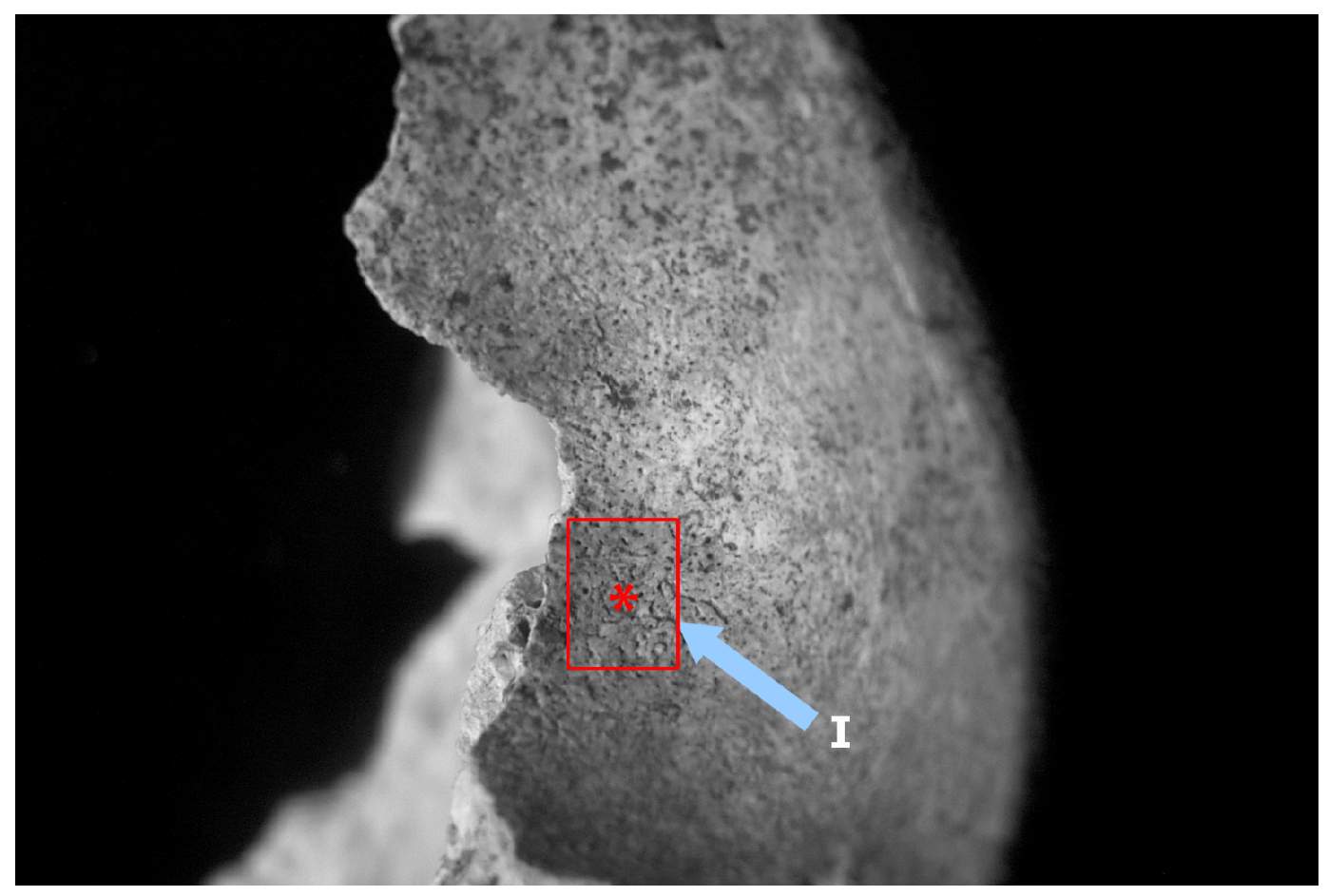

Bild 15: Bau 6, männlich, 35-49 Jahre, Orbitadach links

Fein poröse Oberfläche mit trabekulär konfluierenden Neubildungen, netzartige Gefäßimpressionen (I)

$\rightarrow$ Cribra orbitalia

* Areal der Probenentnahme für Rasterelektronenmikroskopie, siehe Bild 16 


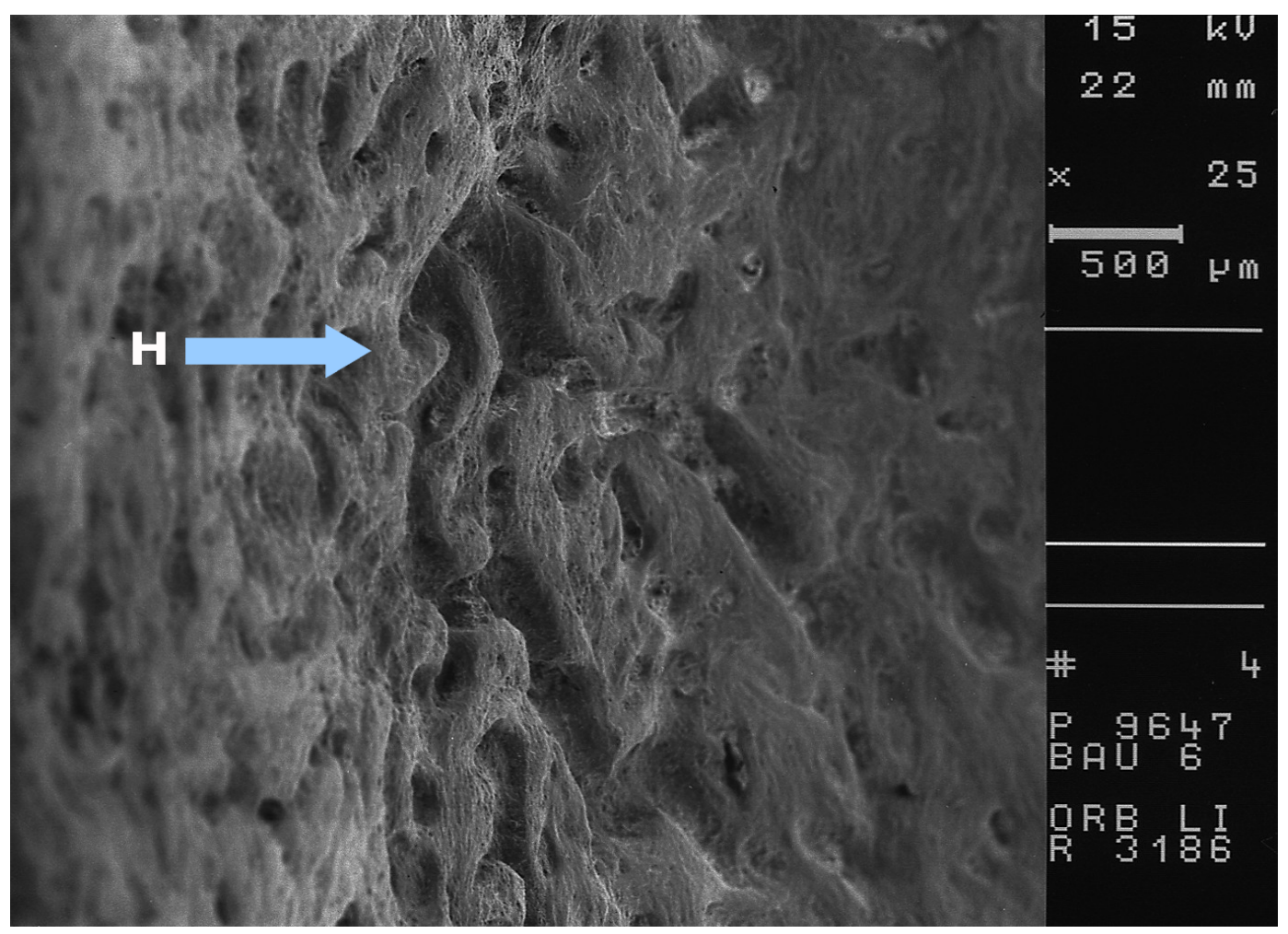

Bild 16: Bau 6, Orbitadach links

Rasterelektronenmikroskopische Aufnahme (REM), Vergrößerung 25x

Intravitale Läsion, Hyperplasie $(\mathrm{H})$ des blutbildenden Knochenmarks als regenerative Reaktion bei chronischer Anämie. Keine sicheren inflammatorische Zeichen. 


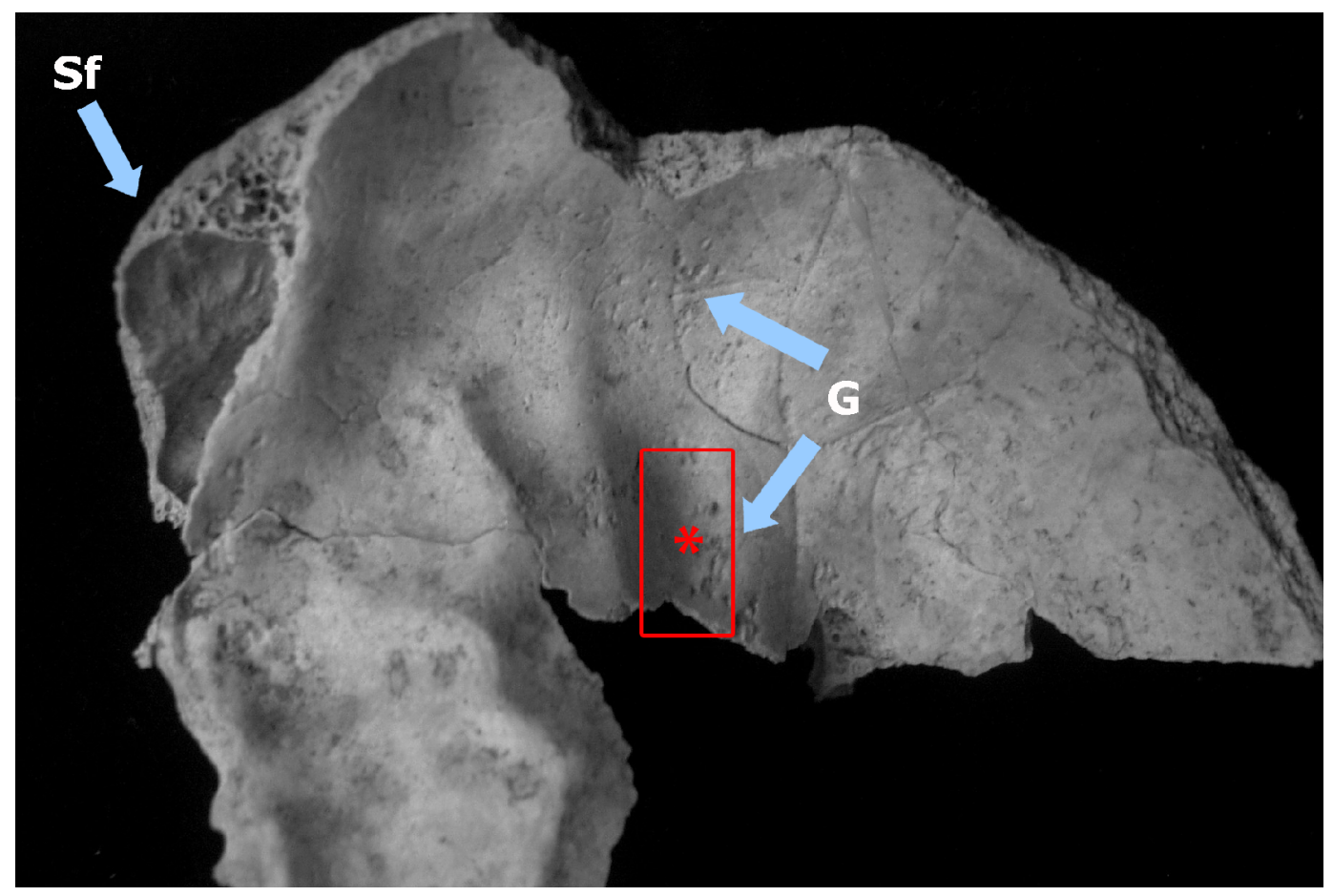

Bild 17: Bau 55, weiblich, 25-30 Jahre Os frontale, Fossa cranii anterior rechts

Teilweise konfluierende mohn- bis pfefferkorngroße Grübchen $(G)$ mit vermehrten Gefäßimpressionen in der Peripherie, verstärkte Impressiones digitatae Nebenbefundlich Sinusitis frontalis (Sf) rechts.

* Areal der Probenentnahme für Rasterelektronenmikroskopie, siehe Bilder 18 a-d 


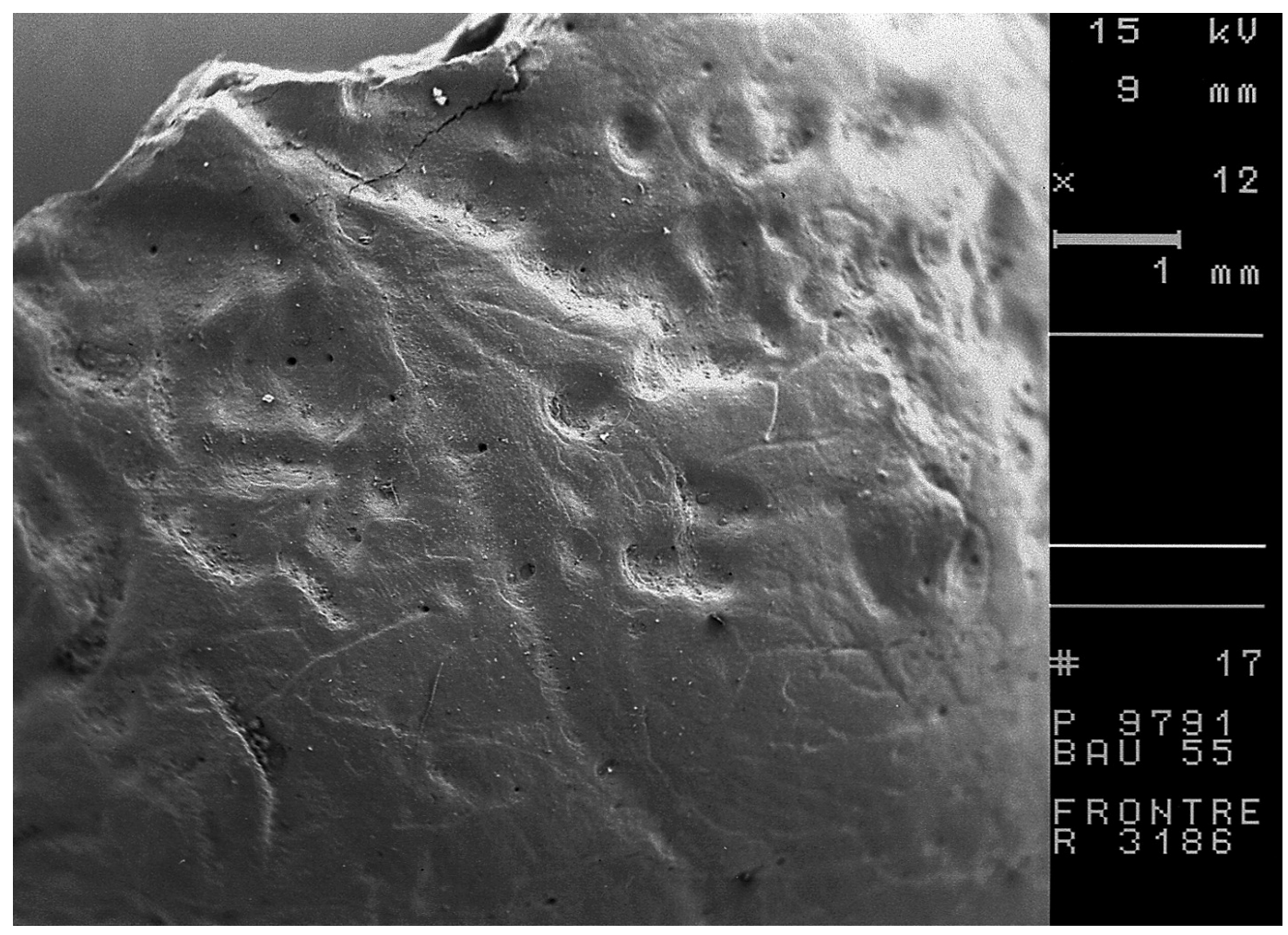

Bild 18a: Bau 55, Os frontale, Fossa cranii anterior rechts

Rasterelektronenmikroskopische Aufnahme (REM), Vergrößerung 12 x

Intravitale drucktrophische Impressionen mit vermehrten Gefäßimpressionen. Keine akut entzündlichen Veränderungen.

$\rightarrow$ Verdacht auf inaktive Leptomeningitis tuberculosa 


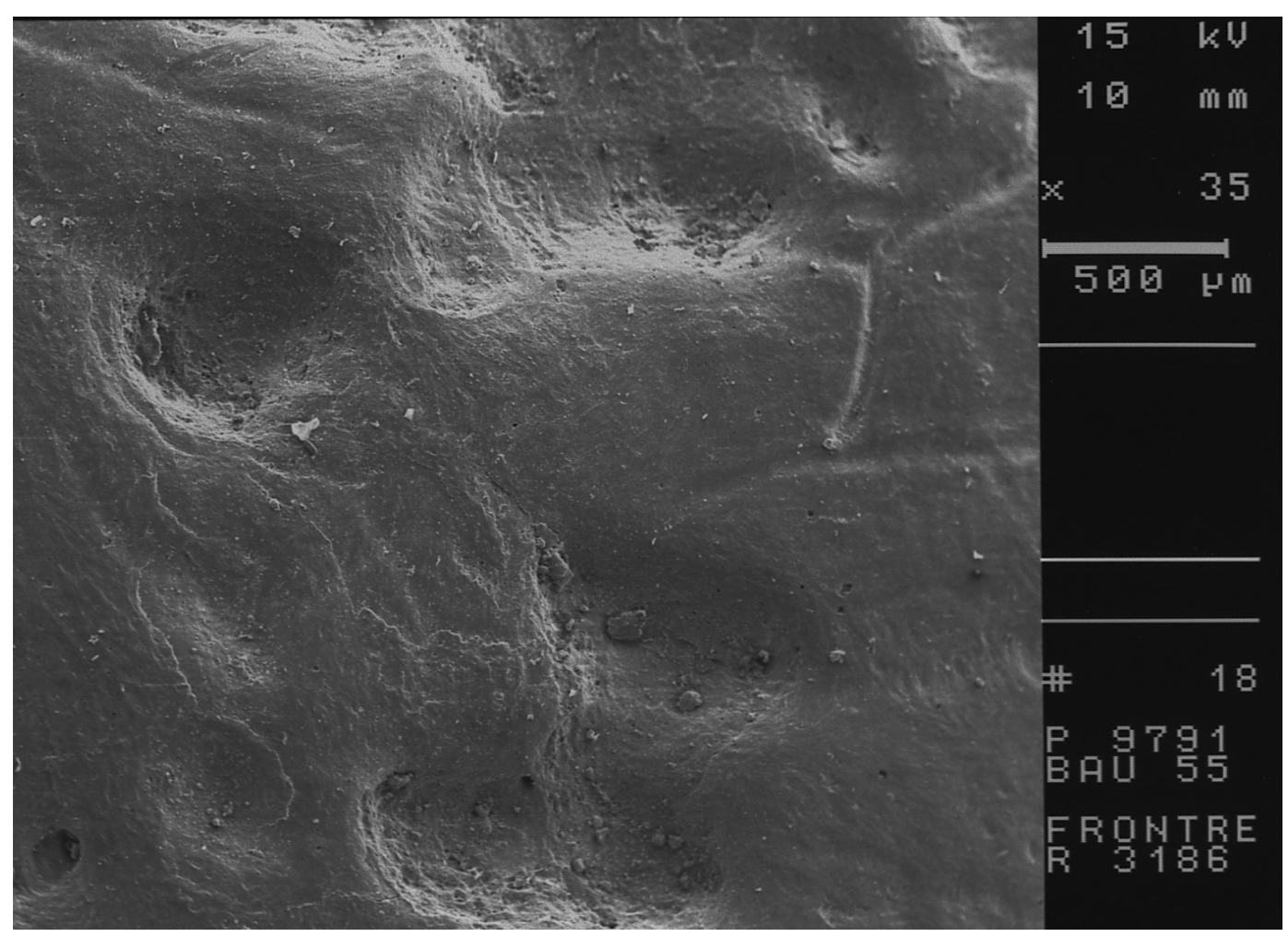

Bild 18b: Bau 55, Os frontale, Fossa cranii anterior rechts

Rasterelektronenmikroskopische Aufnahme (REM), Vergrößerung 35 x

Intravitale drucktrophische Impressionen mit vermehrten Gefäßimpressionen. Keine akut entzündlichen Veränderungen. Vermehrte irreguläre Gefäßimpressionen bei chronisch entzündlichem Prozess. 


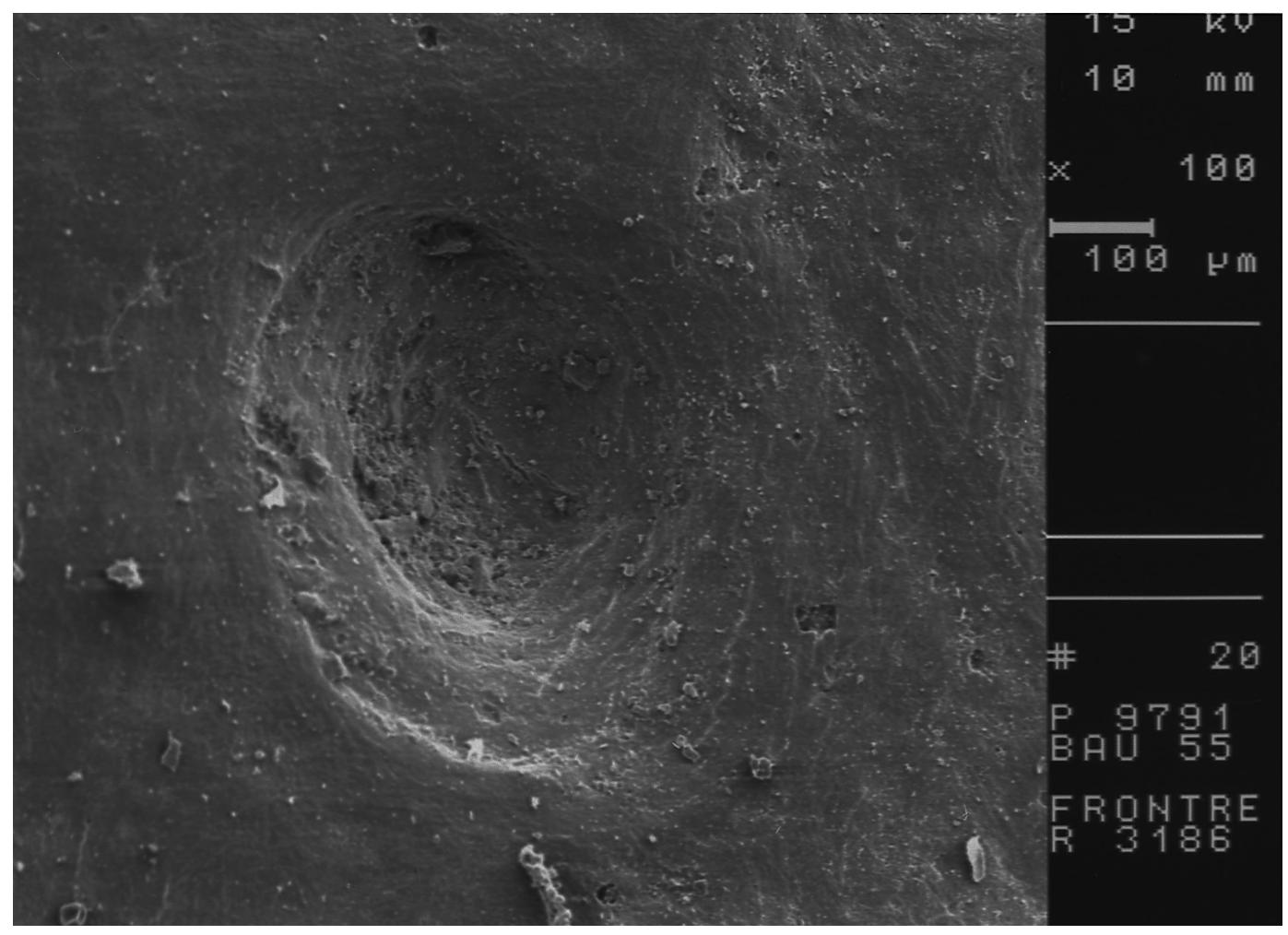

Bild 18c: Bau 55, Os frontale, Fossa cranii anterior rechts

Rasterelektronenmikroskopische Aufnahme (REM), Vergrößerung 100 x

Intravitale Impressionen, am ehesten durch Tuberkel-Granulome bei inaktiver Leptomeningitis tuberculosa. Keine akut entzündlichen Veränderungen. 


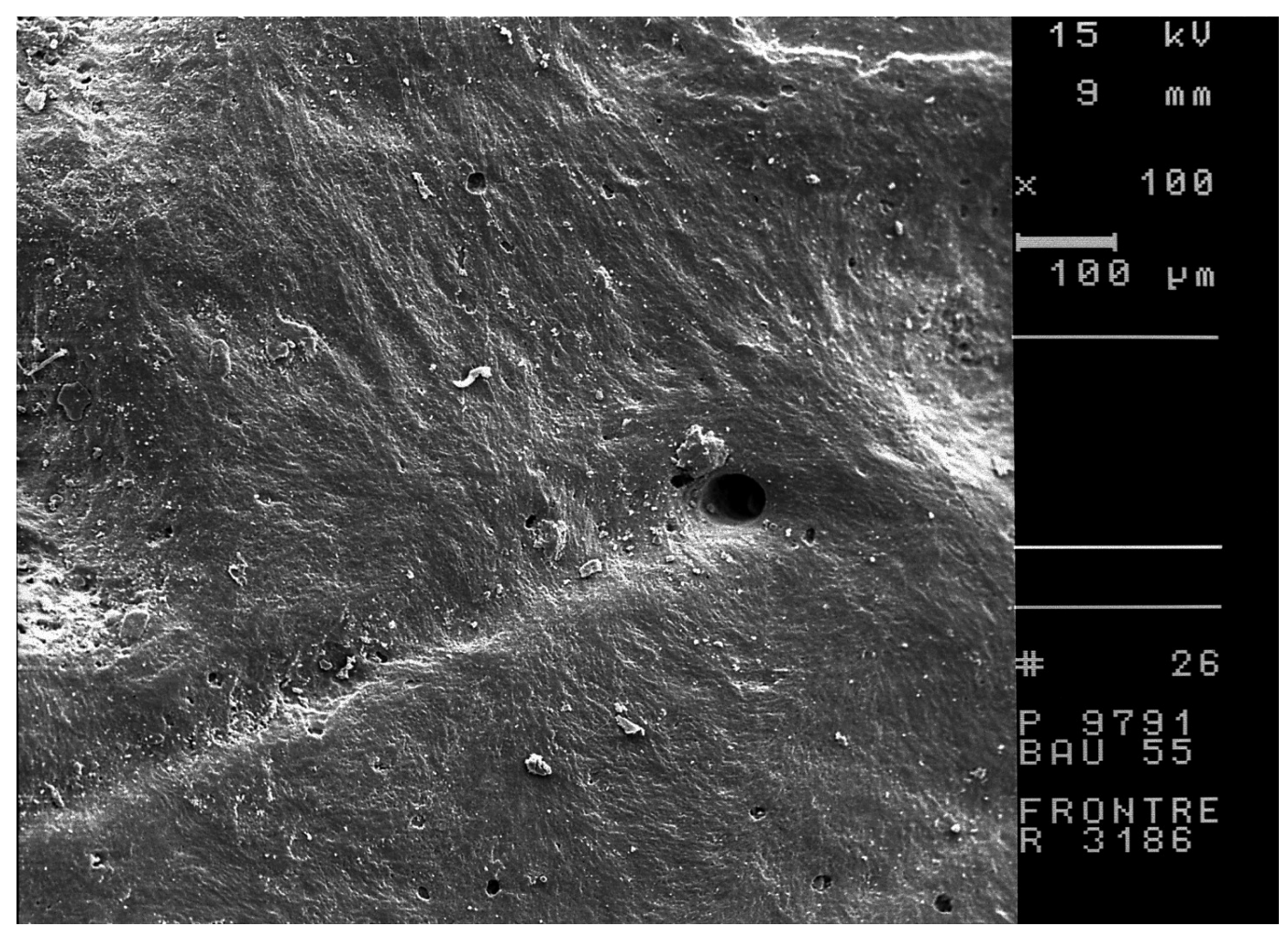

Bild 18d: Bau 55, Os frontale, Fossa cranii anterior rechts

Rasterelektronenmikroskopische Aufnahme (REM), Vergrößerung 100 x

Deutliche intravitale Gefäßimpression aus reizlosem Gefäßforamen als Hinweis auf hyperämisches Areal einer chronischen Entzündung bei Leptomeningitis tuberculosa 


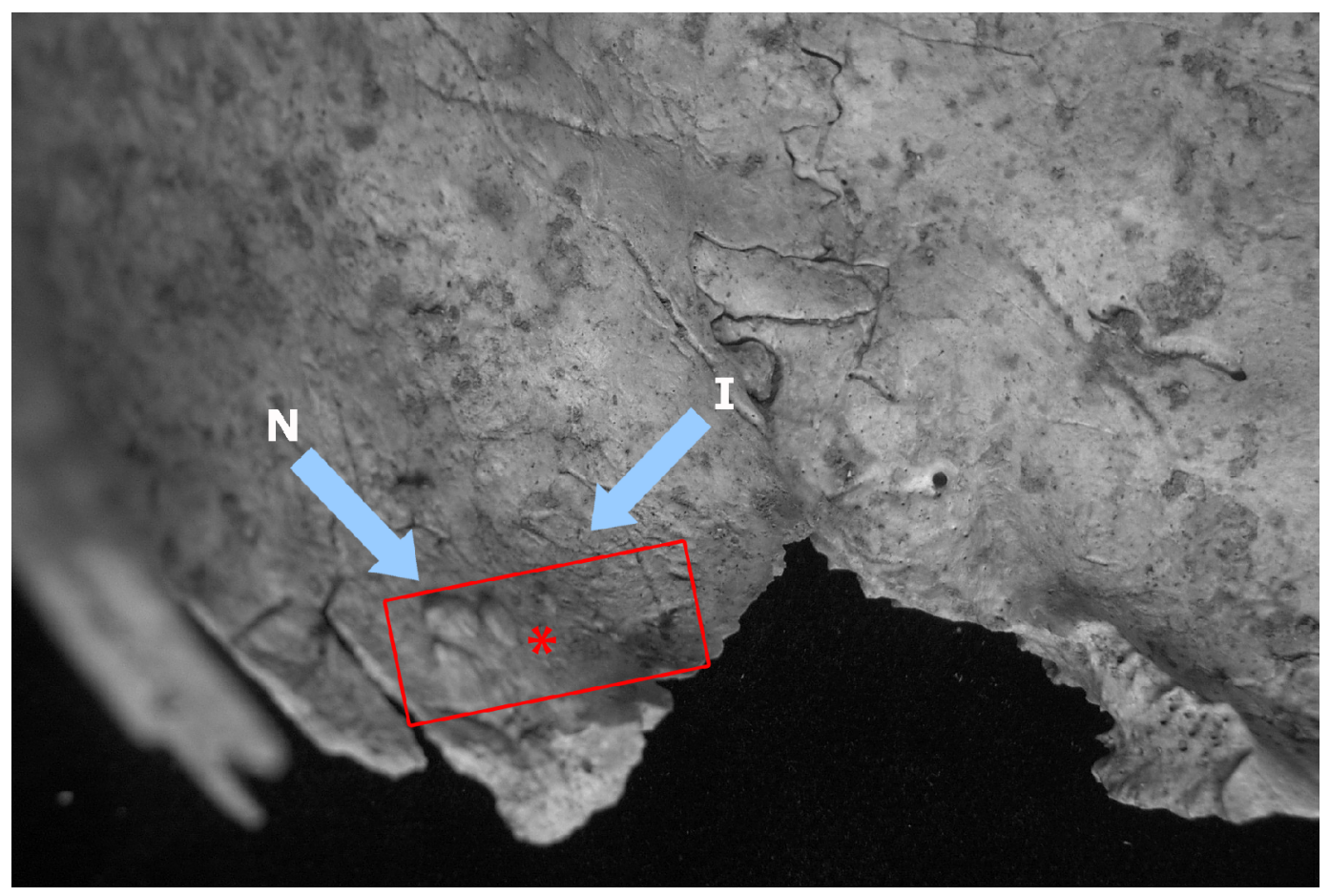

Bild 19: Bau 55, weiblich, 25-30 Jahre Os occipitale, Fossa cranii posterior links

Konfluierende Grübchen mit pfefferkorngroßen Neubildungen $(\mathrm{N})$ und vermehrten Gefäßimpressionen (I).

$\rightarrow$ chronisch entzündlich - hämorrhogischer Prozess, intracranielle TBC

* Areal der Probenentnahme für Rasterelektronenmikroskopie, siehe Bilder 20 a-b 


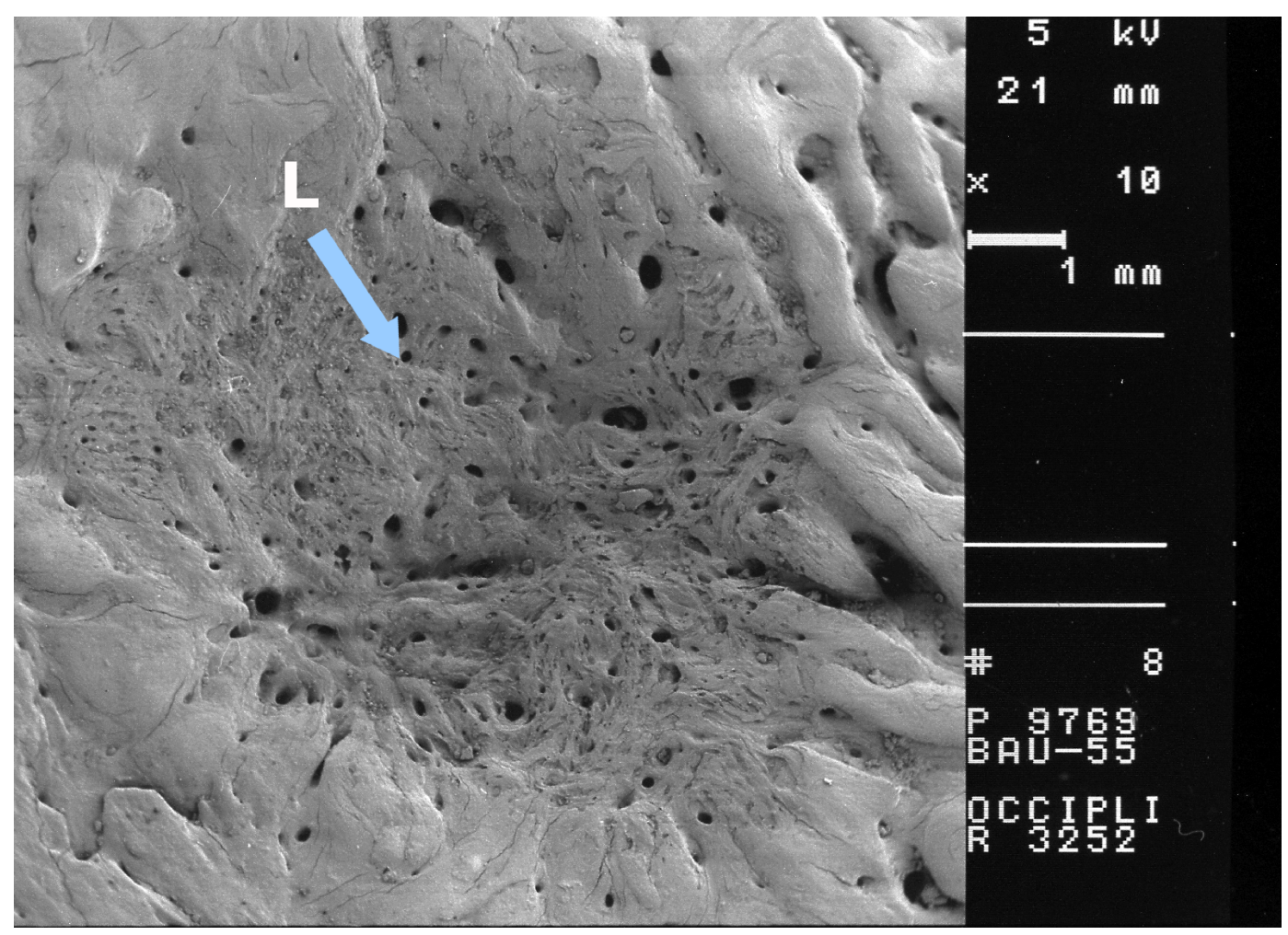

Bild 20a: Bau 55, Os occipitale, Fossa cranii posterior links

Rasterelektronenmikroskopische Aufnahme (REM), Vergrößerung 10 x,

Intravitale Lyse und periphere Neubildungen als Zeichen für chronisch entzündlichen meningealen Prozess.

$\rightarrow$ Verdacht auf Leptomeningitis tuberculosa 


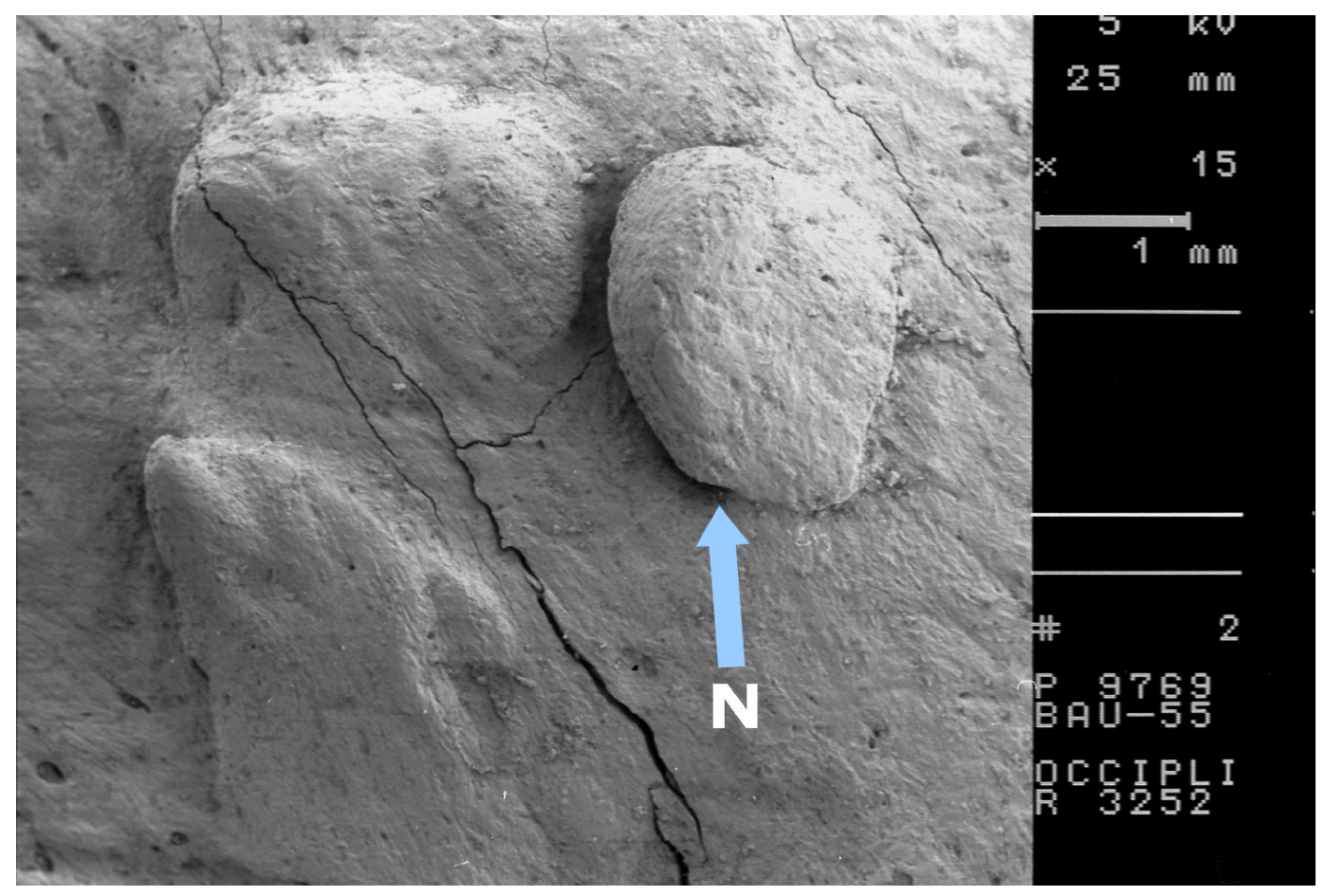

Bild 20b: Bau 55, Os occipitale, Fossa cranii posterior links

Rasterelektronenmikroskopische Aufnahme (REM), Vergrößerung $15 \mathrm{x}$,

Reizlose und in Organisation befindliche Auflagerungen als Hinweis auf abgelaufene entzündliche meningeale Reizung. 


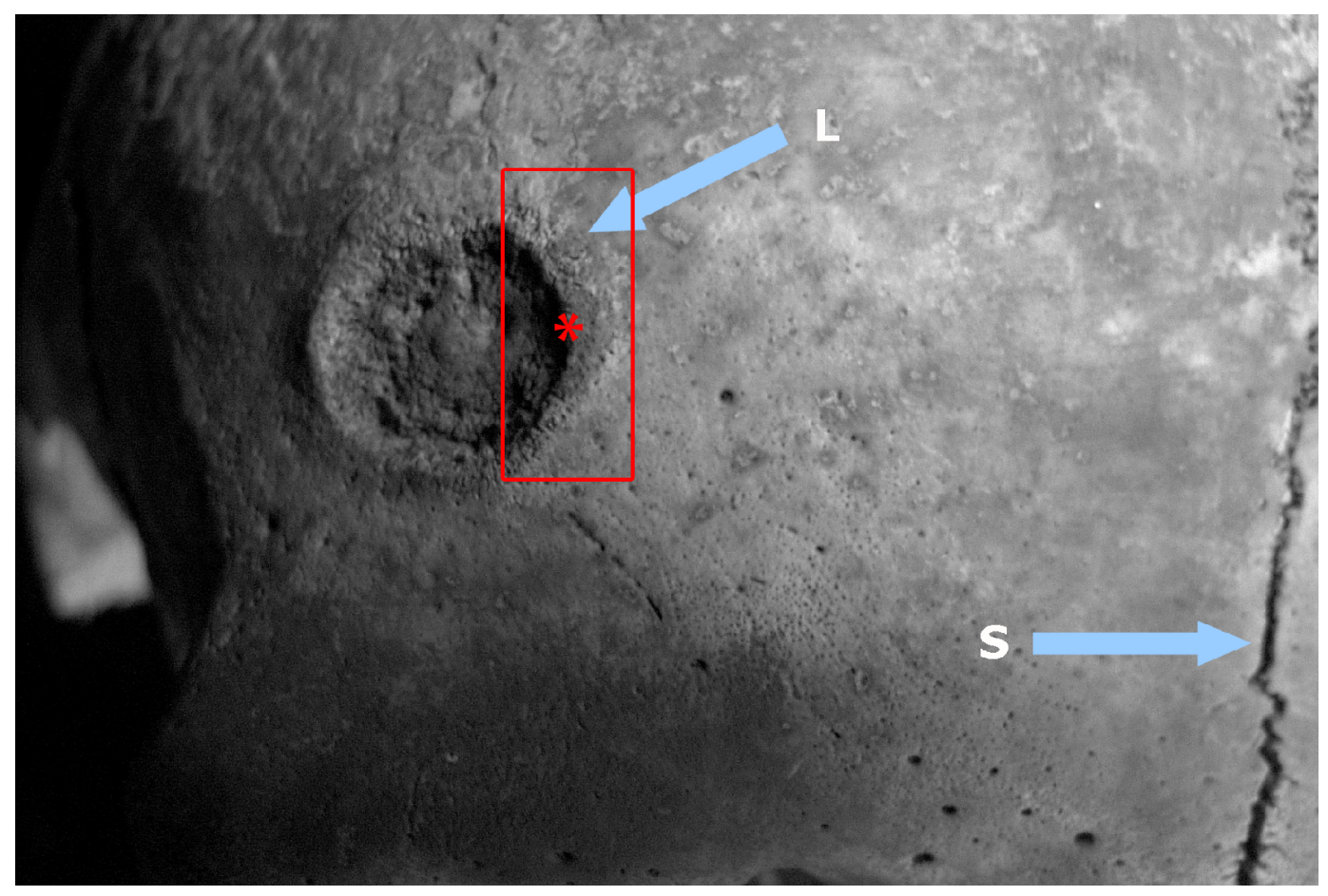

Bild 21: Bau 68, männlich, 20-21 Jahre Os frontale rechts ,Lamina externa

Lytische Läsion mit Randwulst ca. 1,5 cm Durchmesser.

Peripherie der Läsion (L) unauffällig. Nebenbefundlich Sutura metopica (S) als epigenetisches Merkmal ohne Krankheitswert.

* Areal der Probenentnahme für Rasterelektronenmikroskopie, siehe Bild 22 a-d 


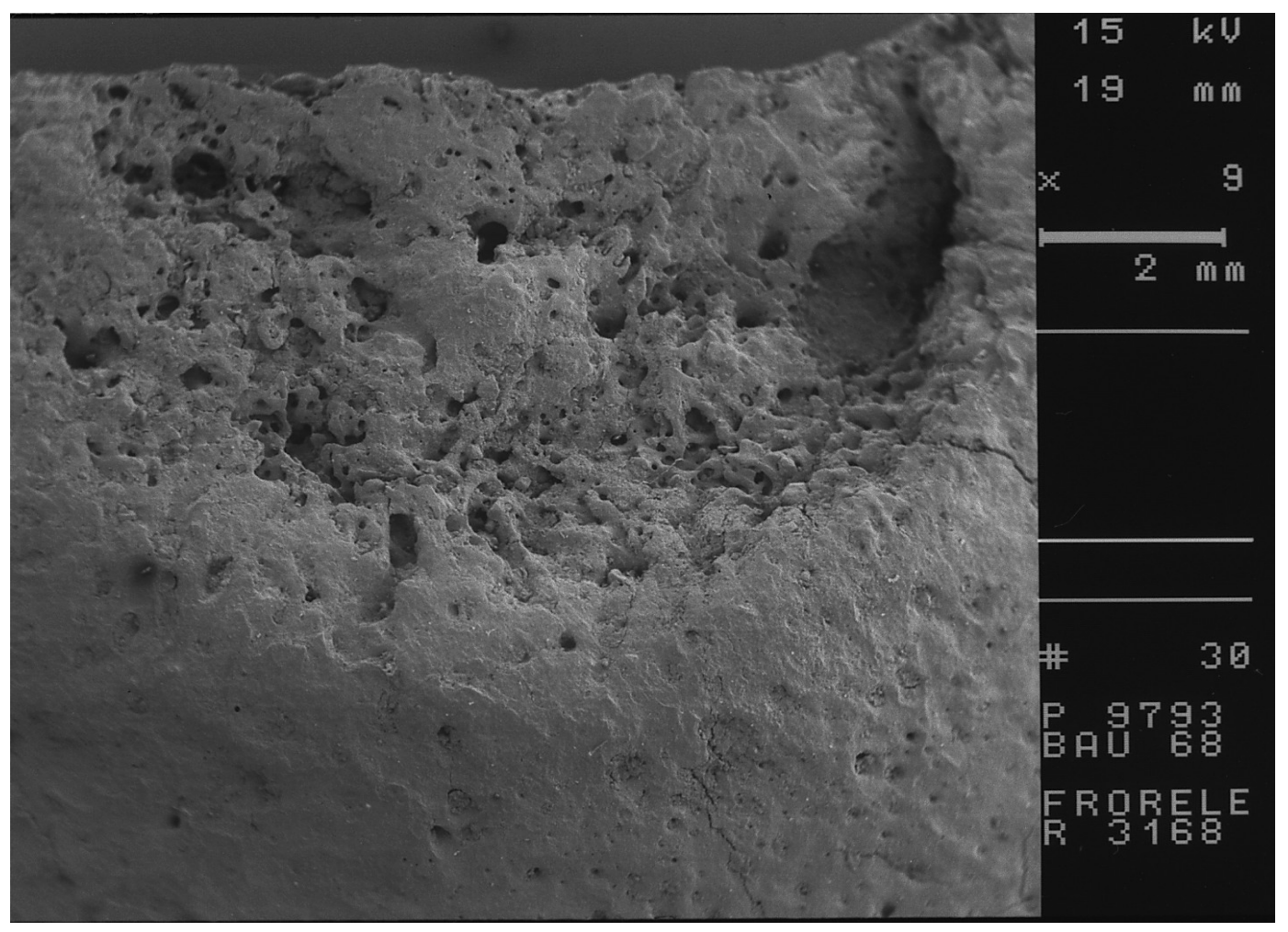

Bild 22a: Bau 68, Os frontale rechts

Rasterelektronenmikroskopische Aufnahme (REM), Vergrößerung $9 \mathrm{x}$

Intravitale Lyse, Prozess bis tief in Diploë reichend.

$\rightarrow$ Metastase? Histiozytosis X? 


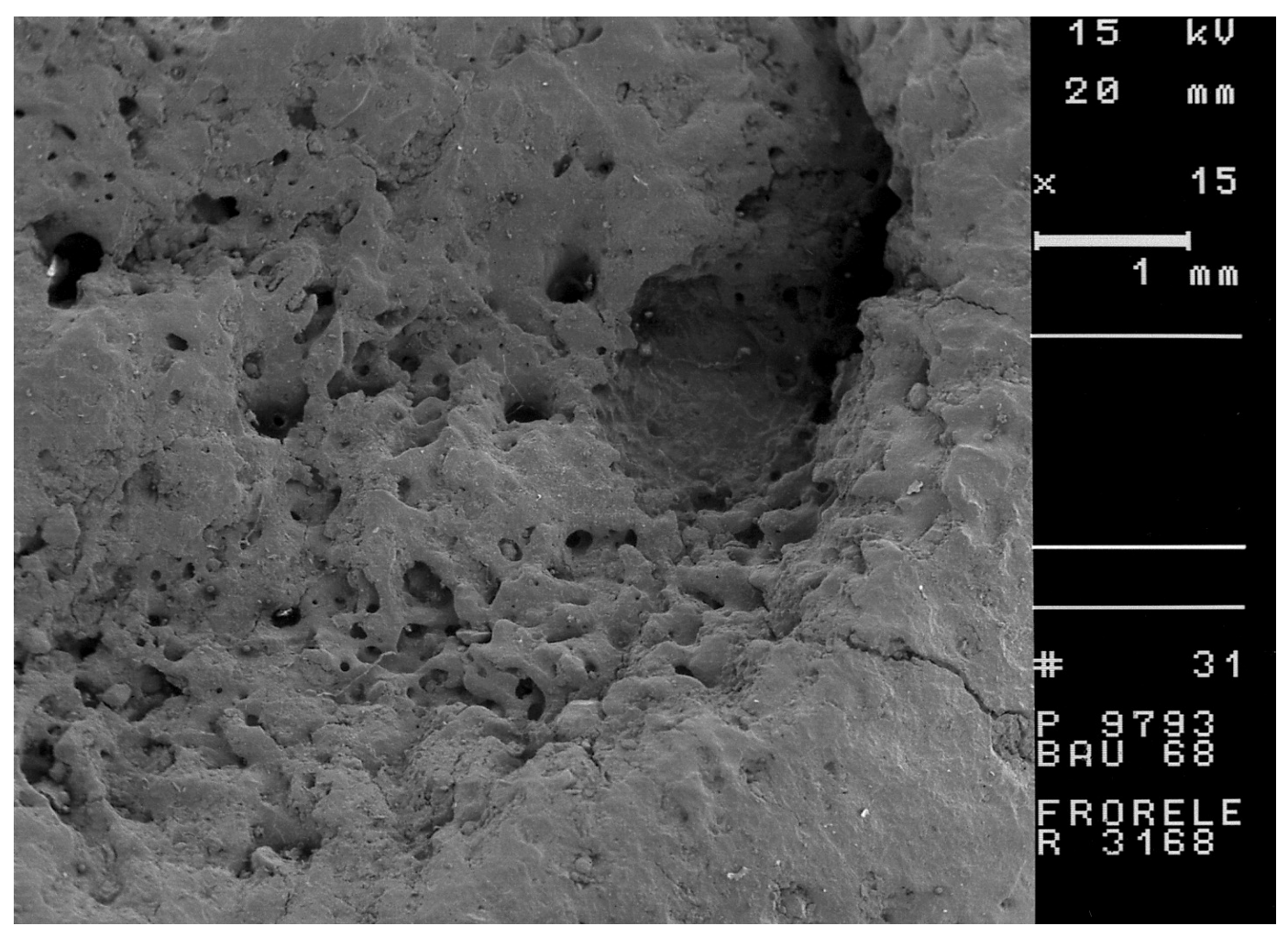

Bild 22b: Bau 68, Os frontale rechts

Rasterelektronenmikroskopische Aufnahme (REM), Vergrößerung 15 x

Intravitale Unterminierung der Corticalis. Gut vaskularisierter Prozess. 


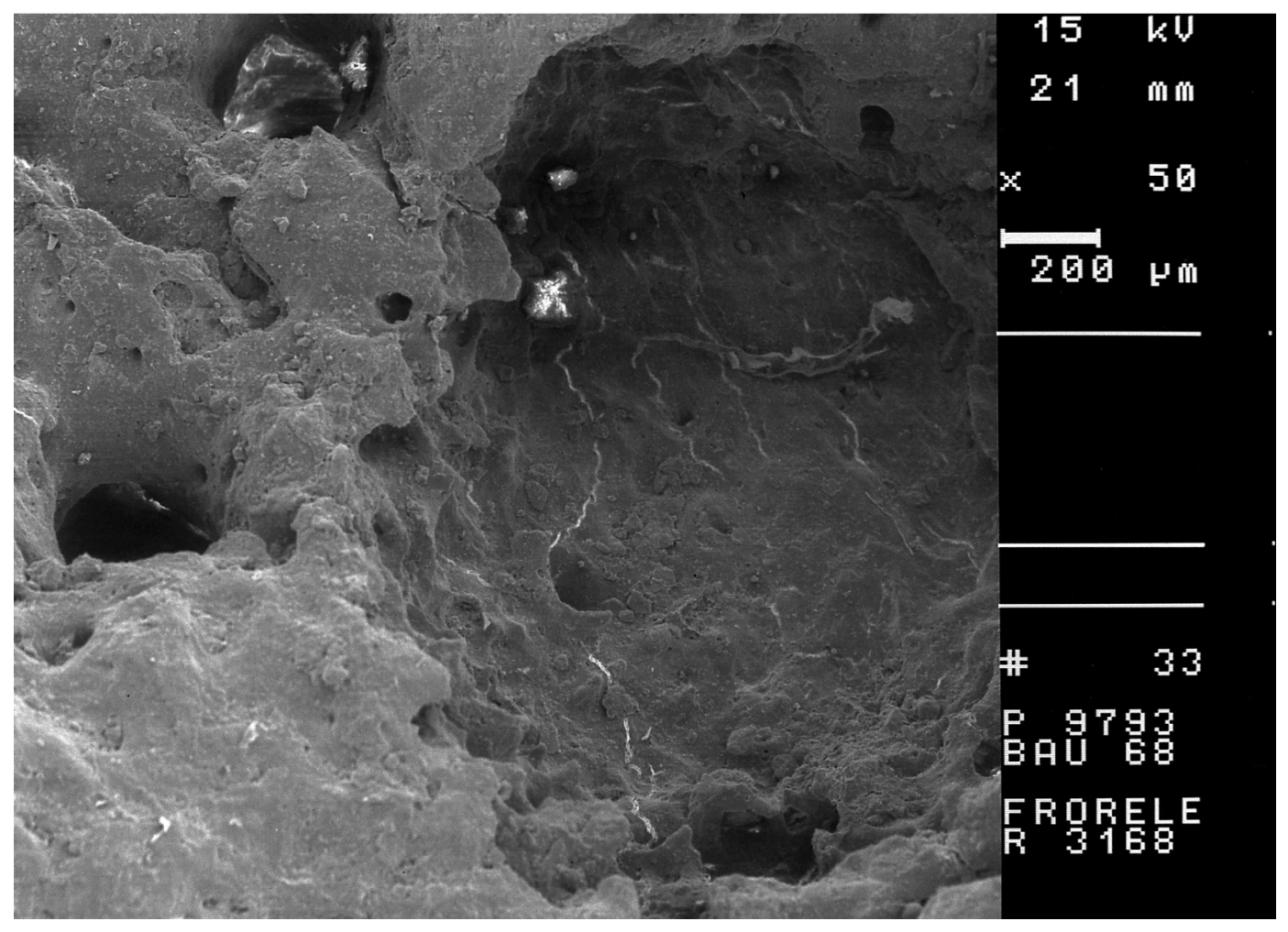

Bild 22c: Bau 68, Os frontale rechts

Rasterelektronenmikroskopische Aufnahme (REM), Vergrößerungen $50 \mathrm{x}$

Intravitale Destruktion der originären Corticalis und Spongiosa durch osteolytischen Prozess. Vereinzelte regenerative Neubildungen als Hinweis auf chronischen Vorgang. 


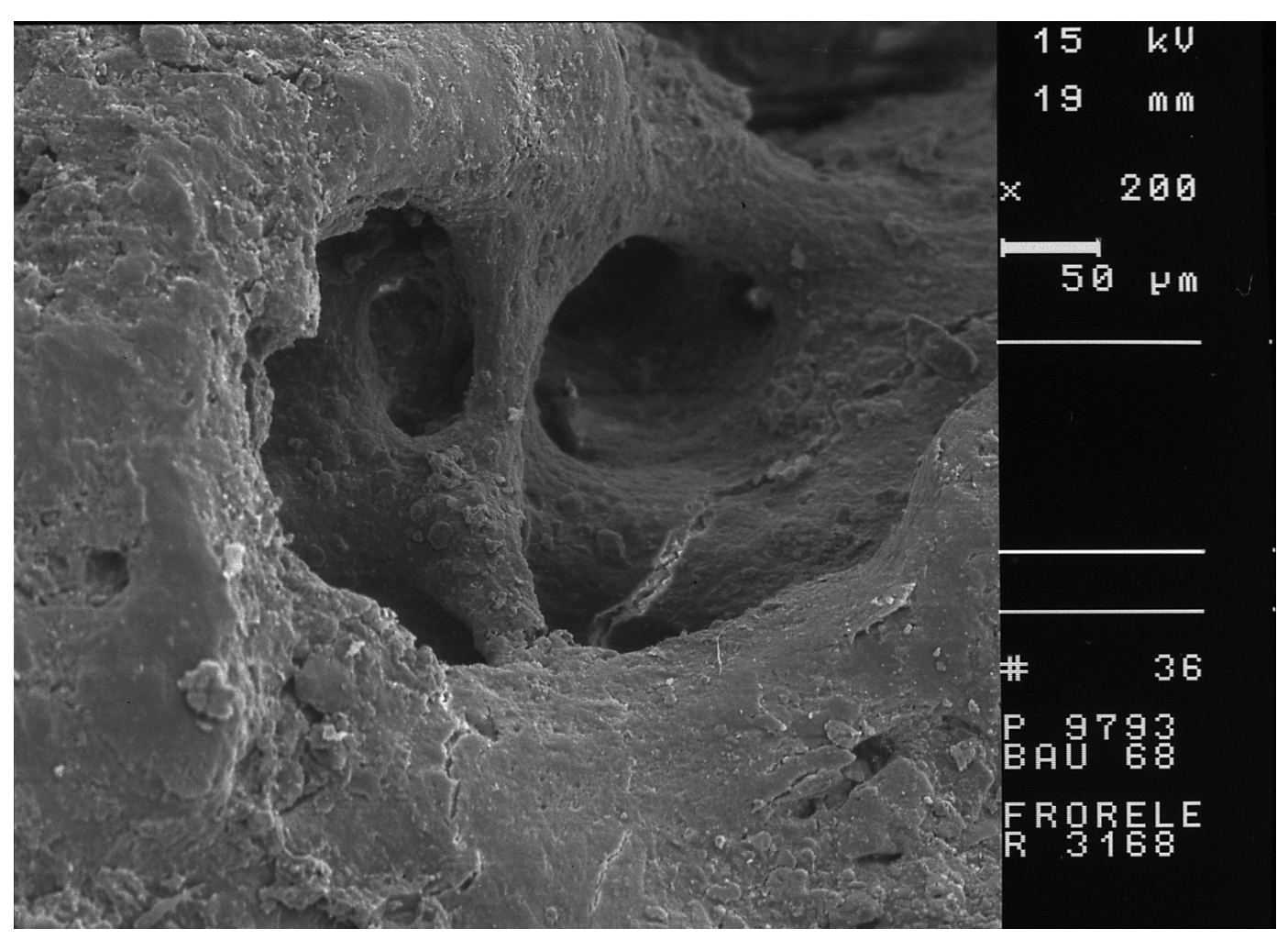

Bild 22d: Bau 68, Os frontale rechts

Rasterelektronenmikroskopische Aufnahme (REM), Vergrößerungen 200 x

Varikös imponierende Gefäßforamina als Hinweis auf gut vaskularisierten Prozess. 


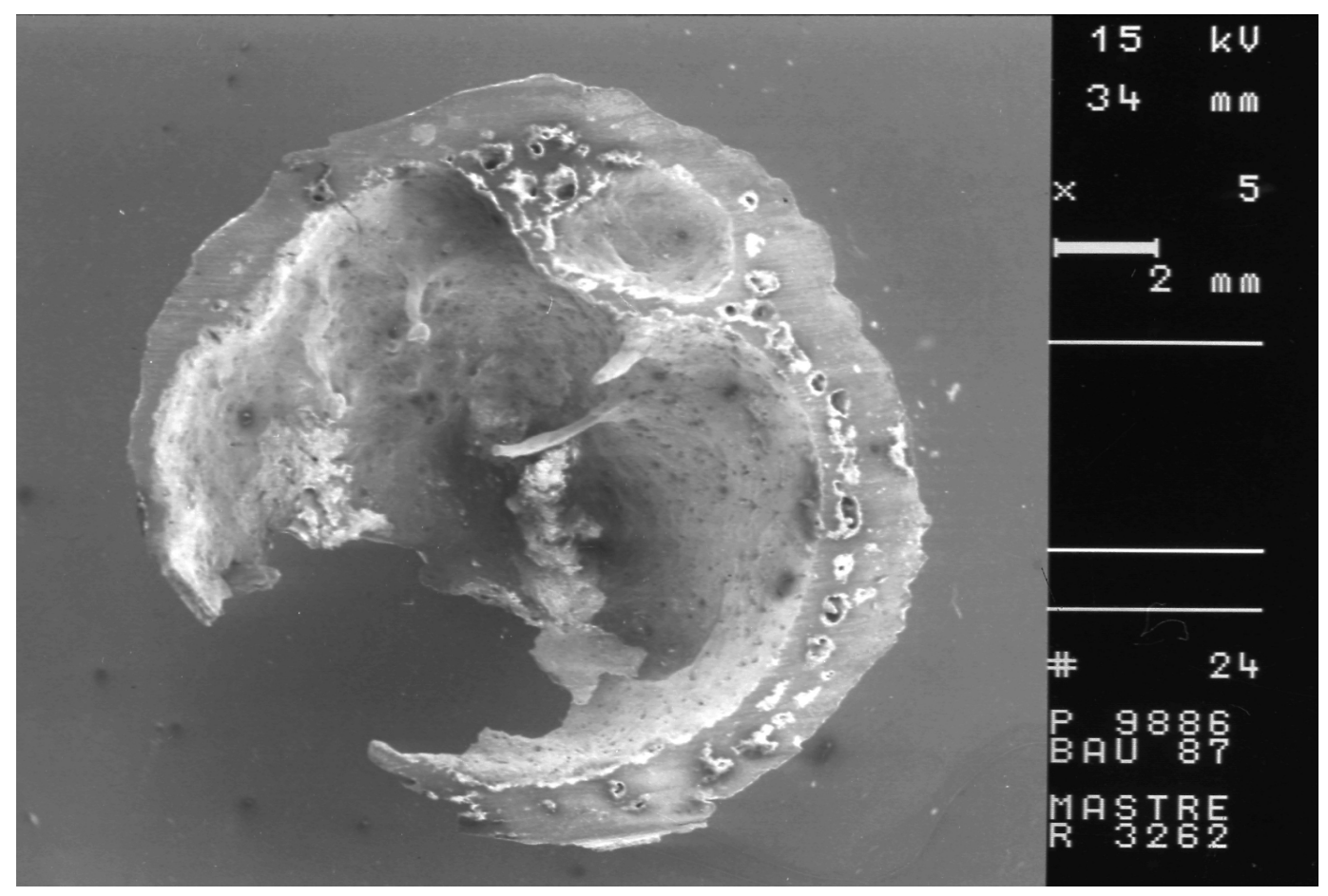

Bild 23: Bau 87, männlich = weiblich, 45-55 Jahre Processus mastoideus rechts, Querschnitt, Übersicht

Rasterelektronenmikroskopische Aufnahme (REM), Vergrößerung 5 x

Ausgeprägte eitrige Mastoiditis mit postmortal vergrößerter Öffnung an Spitze des Warzenfortsatzes bei intravitaler Perforation. Aufgehobene Struktur der Cellulae mastoideae. Am Boden sichtbare intravitale Fistelperforation im Sinne eines Bezold-Abszesses bei Mastoiditis. 


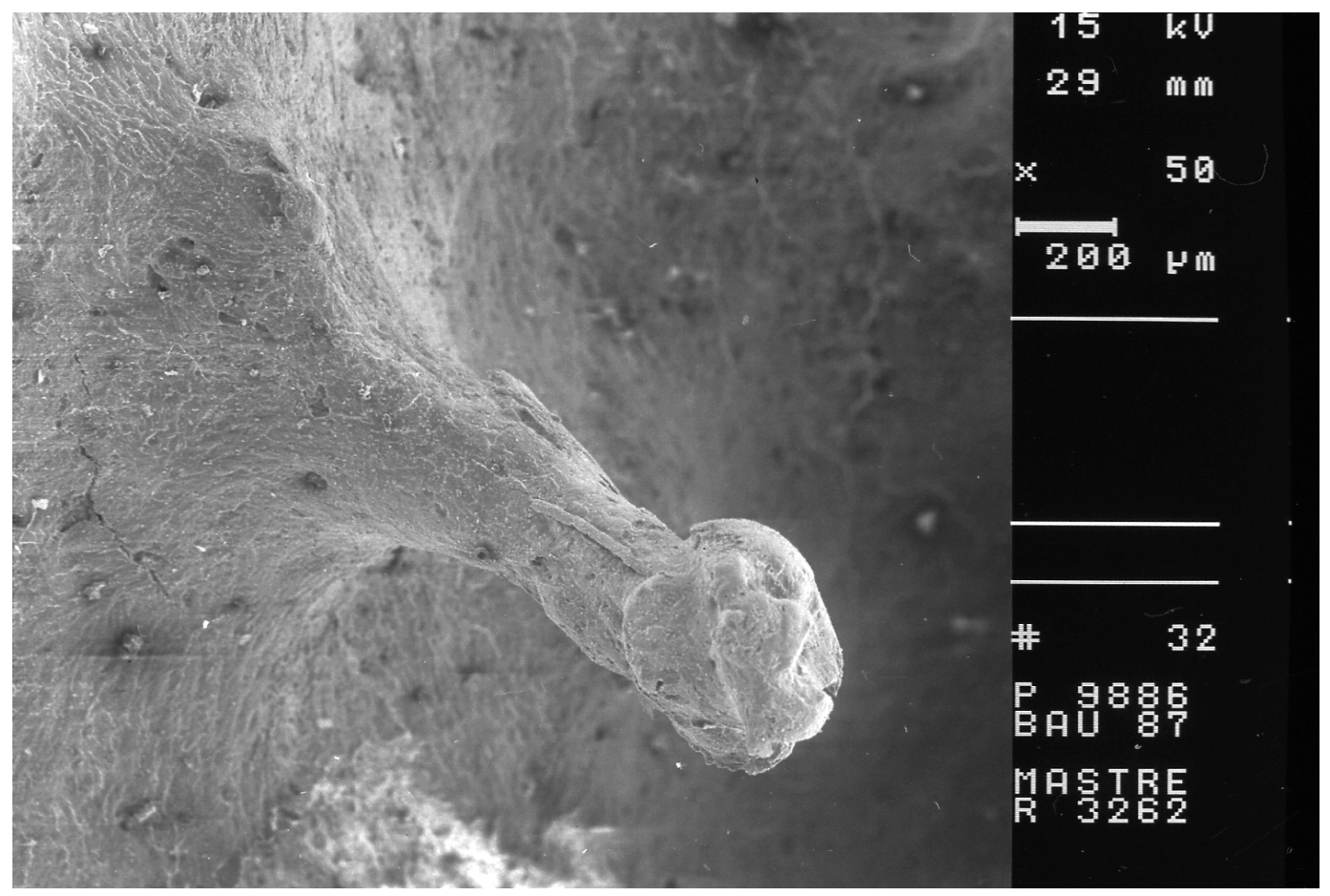

Bild 24a: Bau 87, Processus mastoideus rechts

Rasterelektronenmikroskopische Aufnahme (REM), Vergrößerungen $50 \mathrm{x}$

Narbiges Residuum nach intravitaler Einschmelzung der Cellulae mastoideae bei eitriger Mastoiditis. 


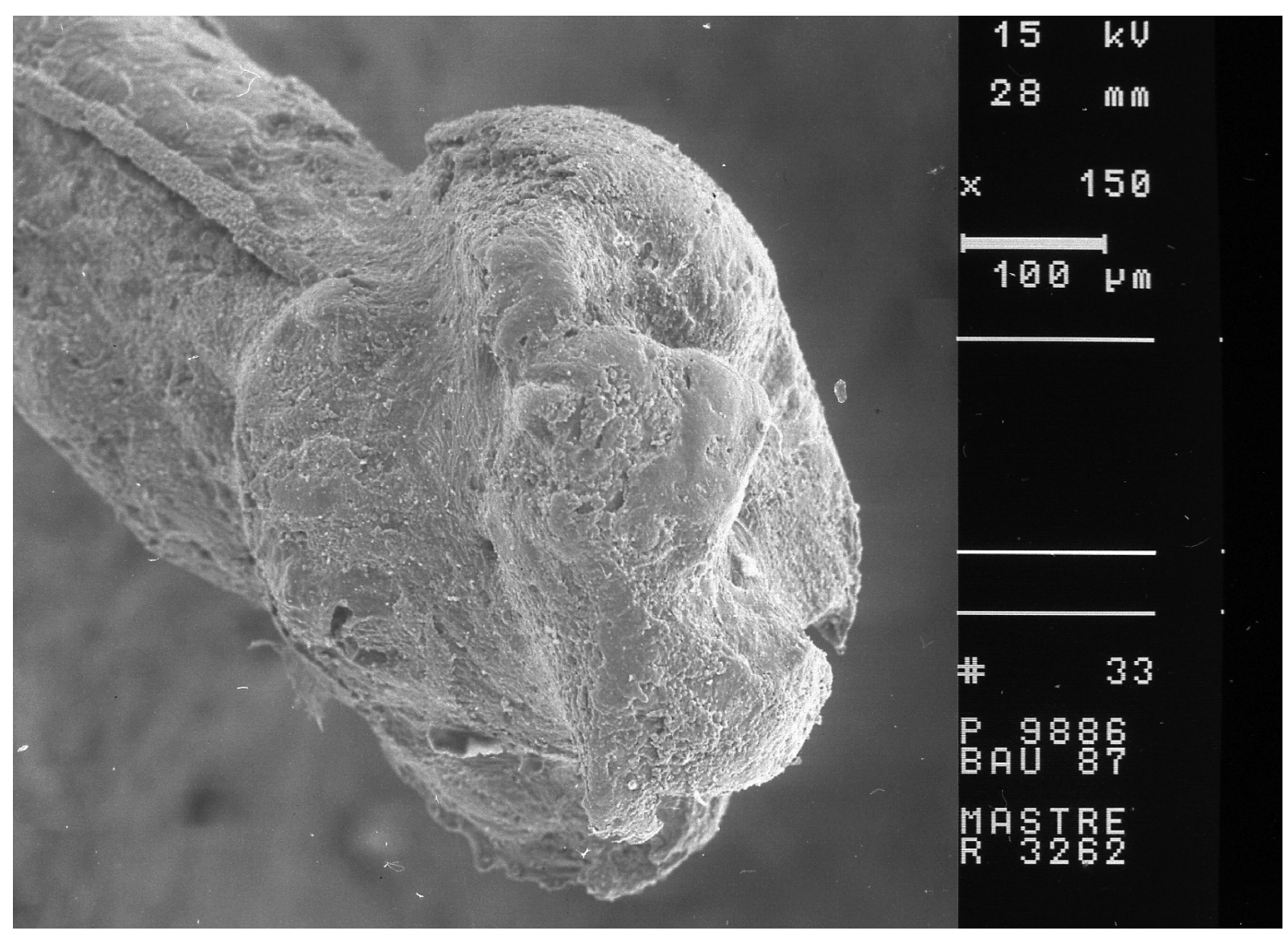

Bild 24b: Bau 87, Processus mastoideus rechts

Rasterelektronenmikroskopische Aufnahme (REM), Vergrößerungen $150 \mathrm{x}$

Gefäßimpression auf vernarbter Oberfläche nach Einschmelzung der Septen als Hinweis auf chronischen Prozess. 


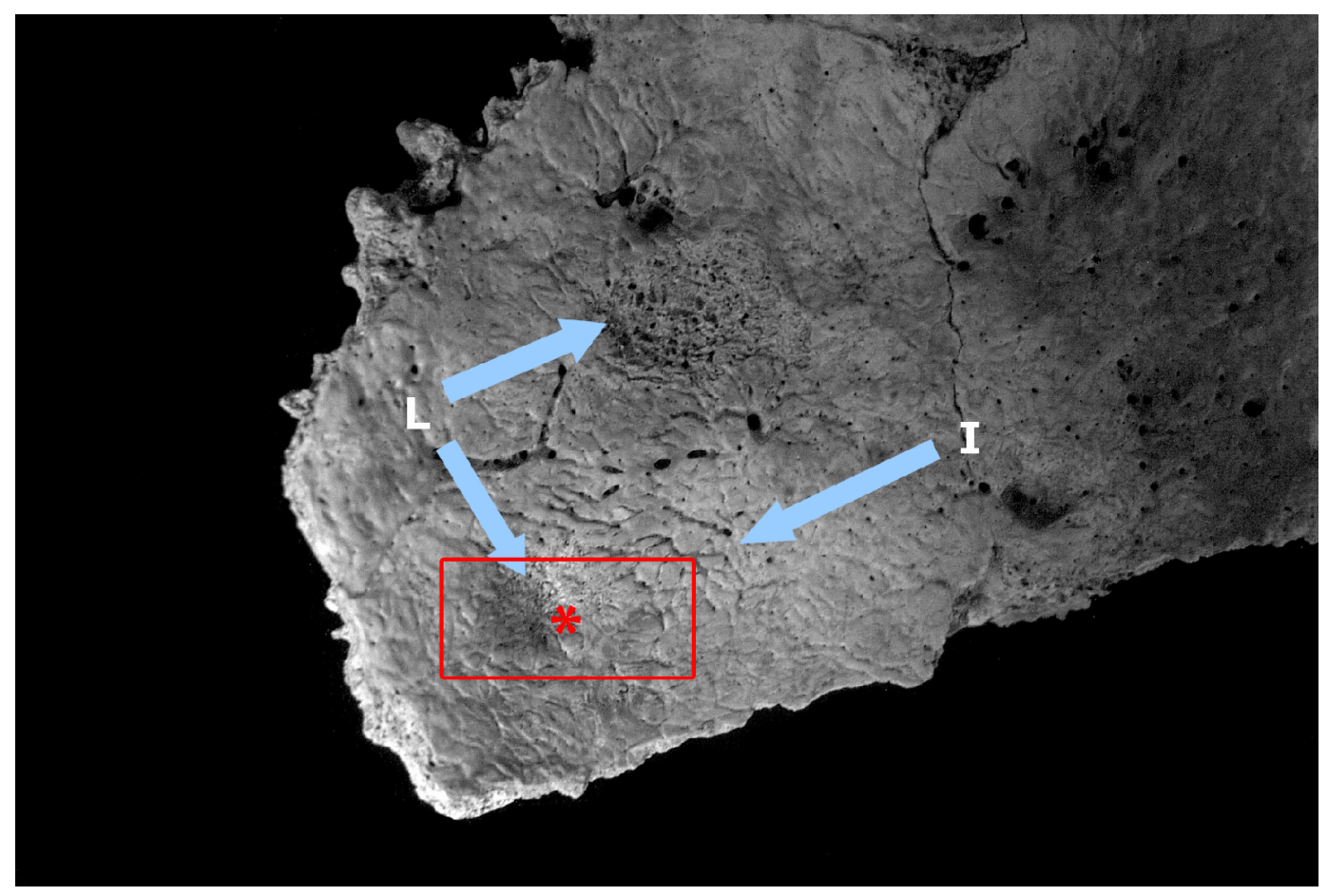

Bild 25: Bau 87, männlich = weiblich, 45-55 Jahre Os occipitale links,

Squama occipitalis, Lamina interna

Ausgeprägte Gefäßimpressionen (I) in plattenartigen Neubildungen neben partiell akut osteolytischen Arealen (L) in Fossa cranii posterior. Vereinzelte reizlose pfefferkorngroße Grübchen

$\rightarrow$ chronische meningeale Reizung, Verdacht auf akut exacerbierte intracranielle $\mathrm{TBC}$

* Areal der Probenentnahme für Rasterelektronenmikroskopie, siehe Bilder 26 a-b 


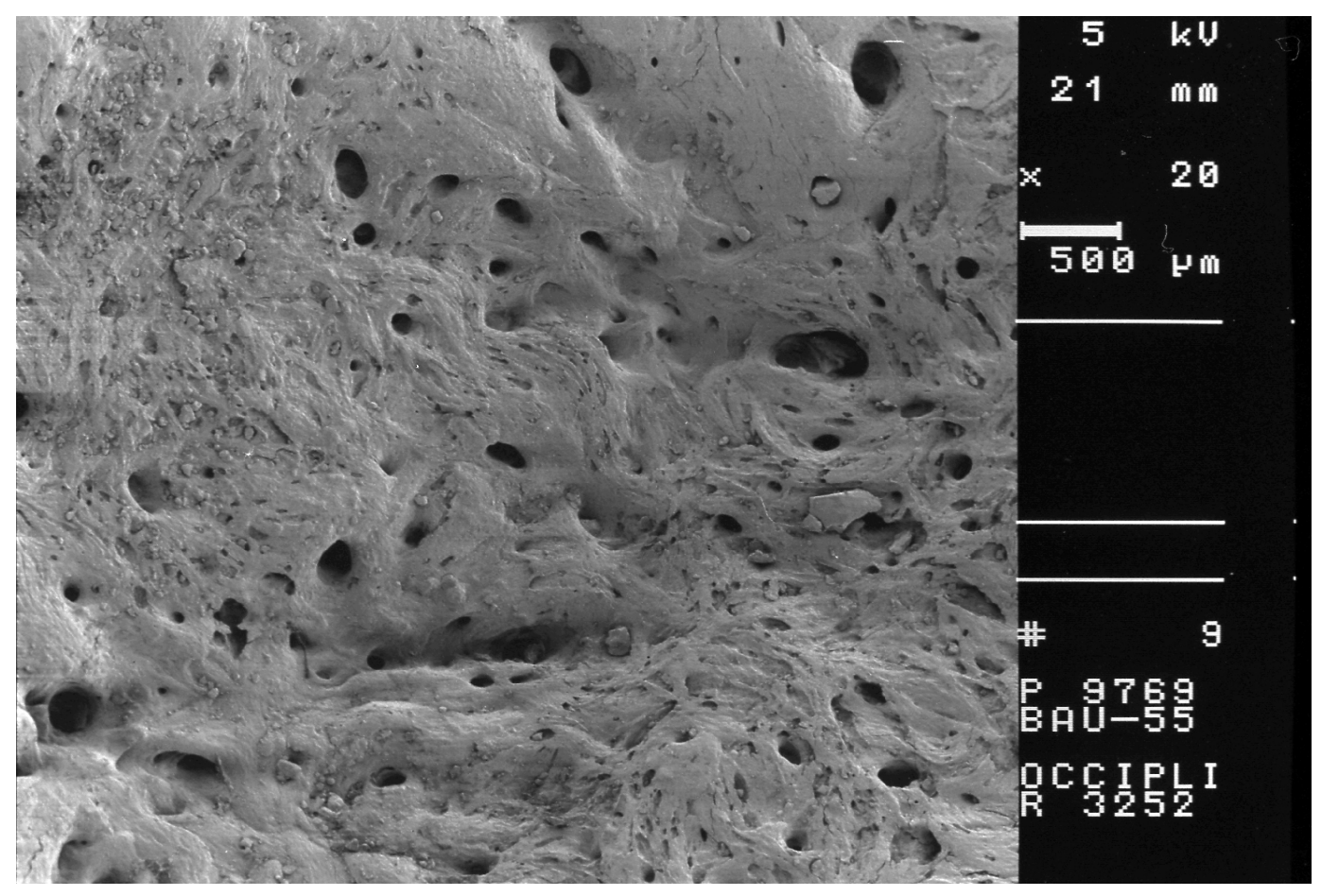

Bild 26a: Bau 87, Os occipitale links, Squama occipitalis, Lamina interna

Rasterelektronenmikroskopische Aufnahme (REM), Vergrößerungen 20 x

Intravitaler osteolytischer Prozess in Nachbarschaft einer chronischen meningealen Reizung.

$\rightarrow$ akut exacerbierter chronisch meningealer Prozess, Verdacht auf Leptomeningitis tuberculosa 


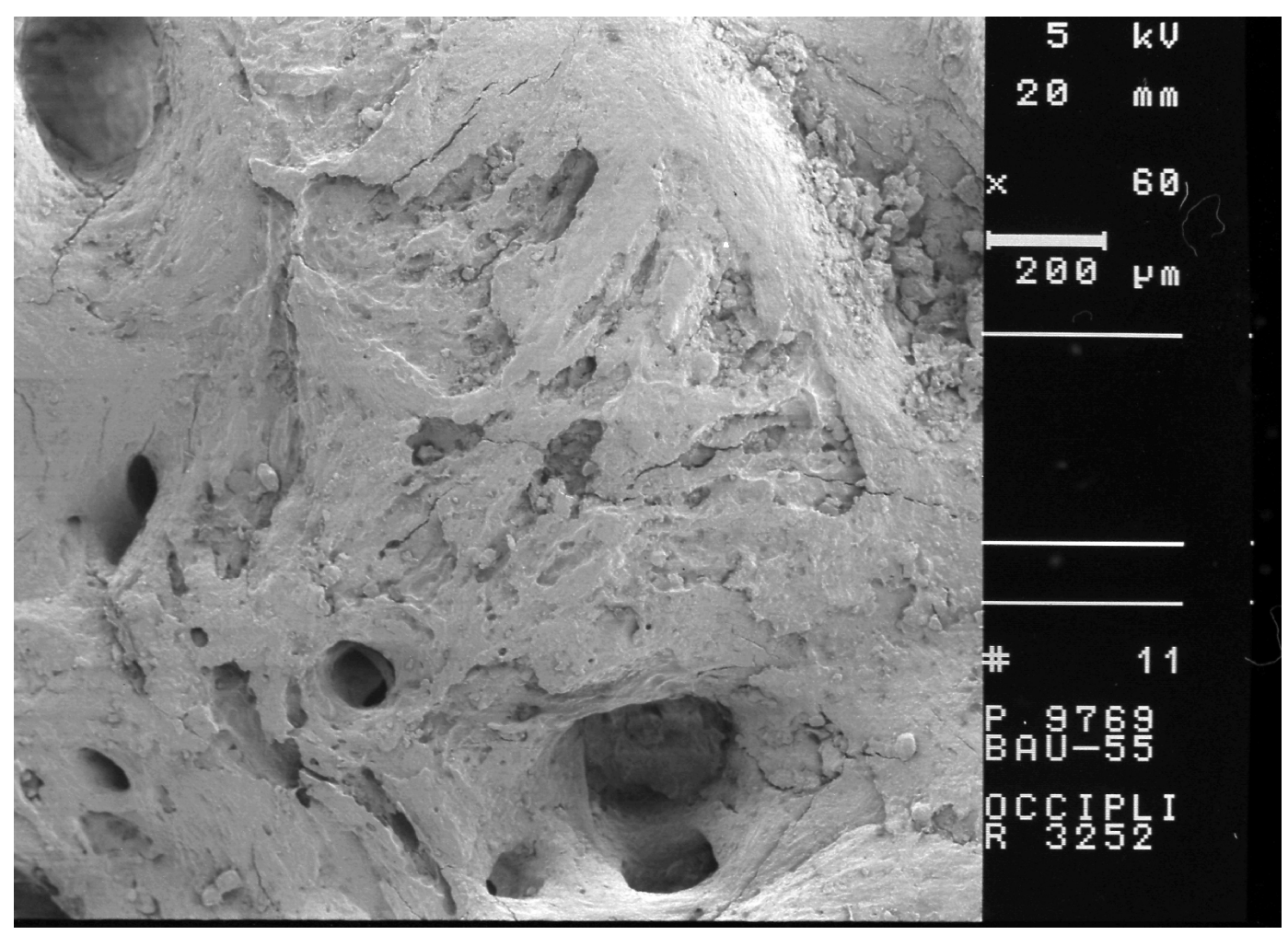

Bild 26b: Bau 87, Os occipitale links, Squama occipitalis, Lamina interna

Rasterelektronenmikroskopische Aufnahme (REM), Vergrößerung 60 x

Vereinzelt in Organisation befindliche Auflagerungen als Hinweis auf chronische meningeale Reizung, gut vaskularisierter Prozess. 


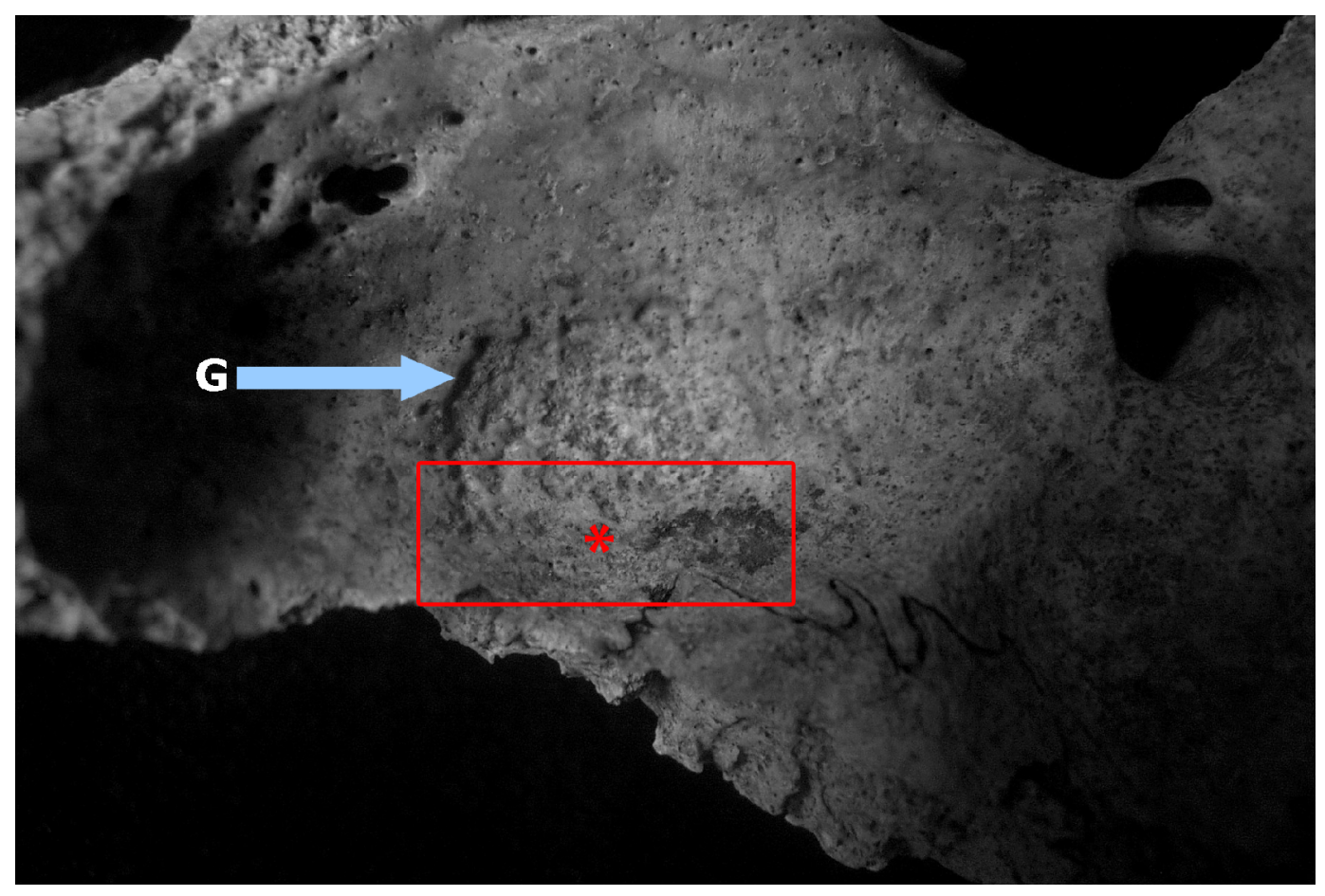

Bild 27: Bau 93, männlich = weiblich, 23-25 Jahre Fossa cranii media, Ala major ossis sphenoidalis links

Konfluierende mohn- bis pfefferkorngroße Grübchen $(\mathrm{G})$ mit poröser Oberfläche. Chronische meningeale Reizung, Verdacht auf Leptomeningitis tuberculosa

* Areal der Probenentnahme für Rasterelektronenmikroskopie, siehe Bilder 28 a-d 


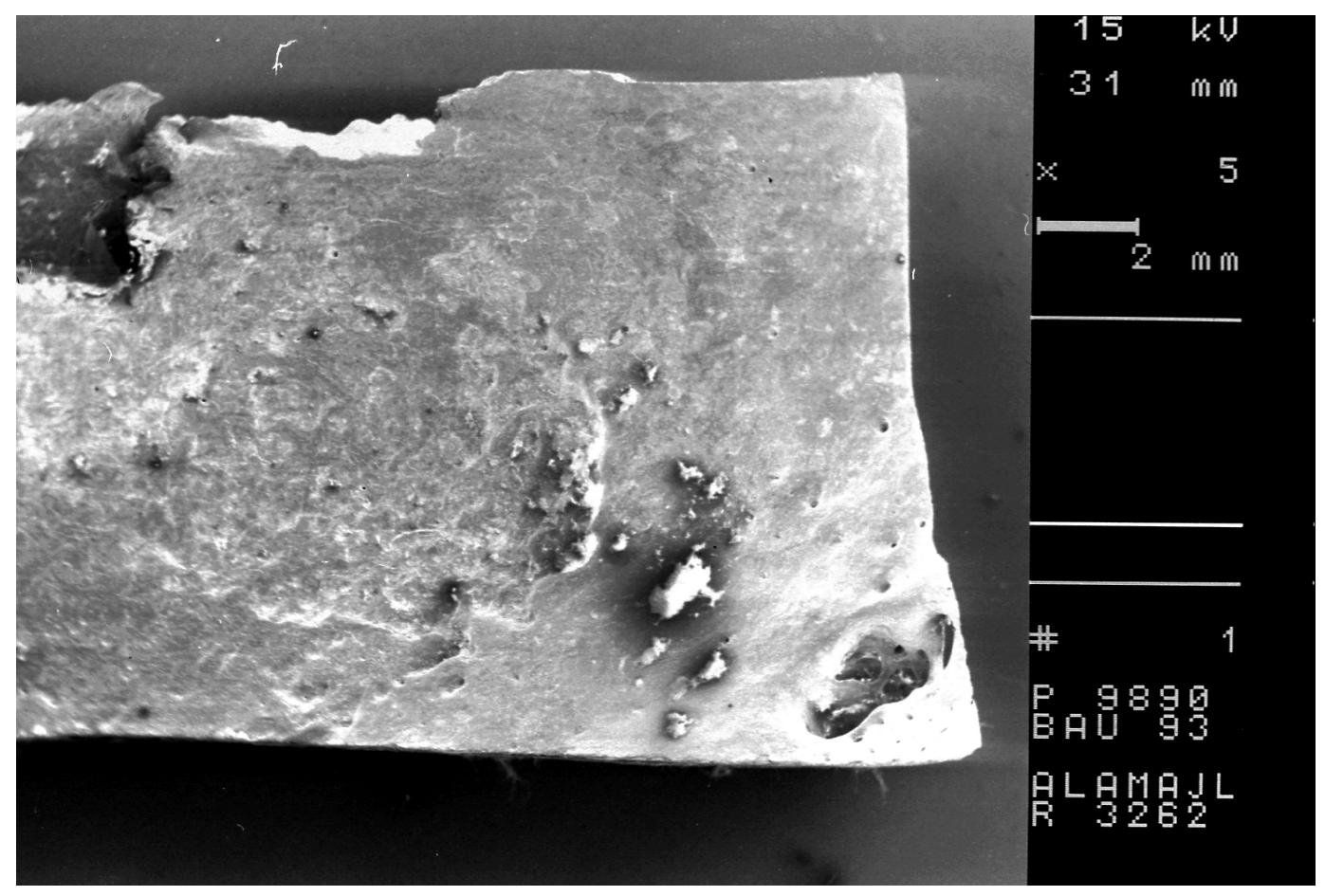

Bild 28a: Bau 93 Fossa cranii media, Ala major ossis sphenoidalis links

Rasterelektronenmikroskopische Aufnahme (REM), Vergrößerungen $5 \mathrm{x}$

Intravitaler chronischer Entzündungsprozess. Vereinzelt postmortal erodierte Knochensubstanz am Probenrand.

$\rightarrow$ chronische Leptomeningitis tuberculosa 


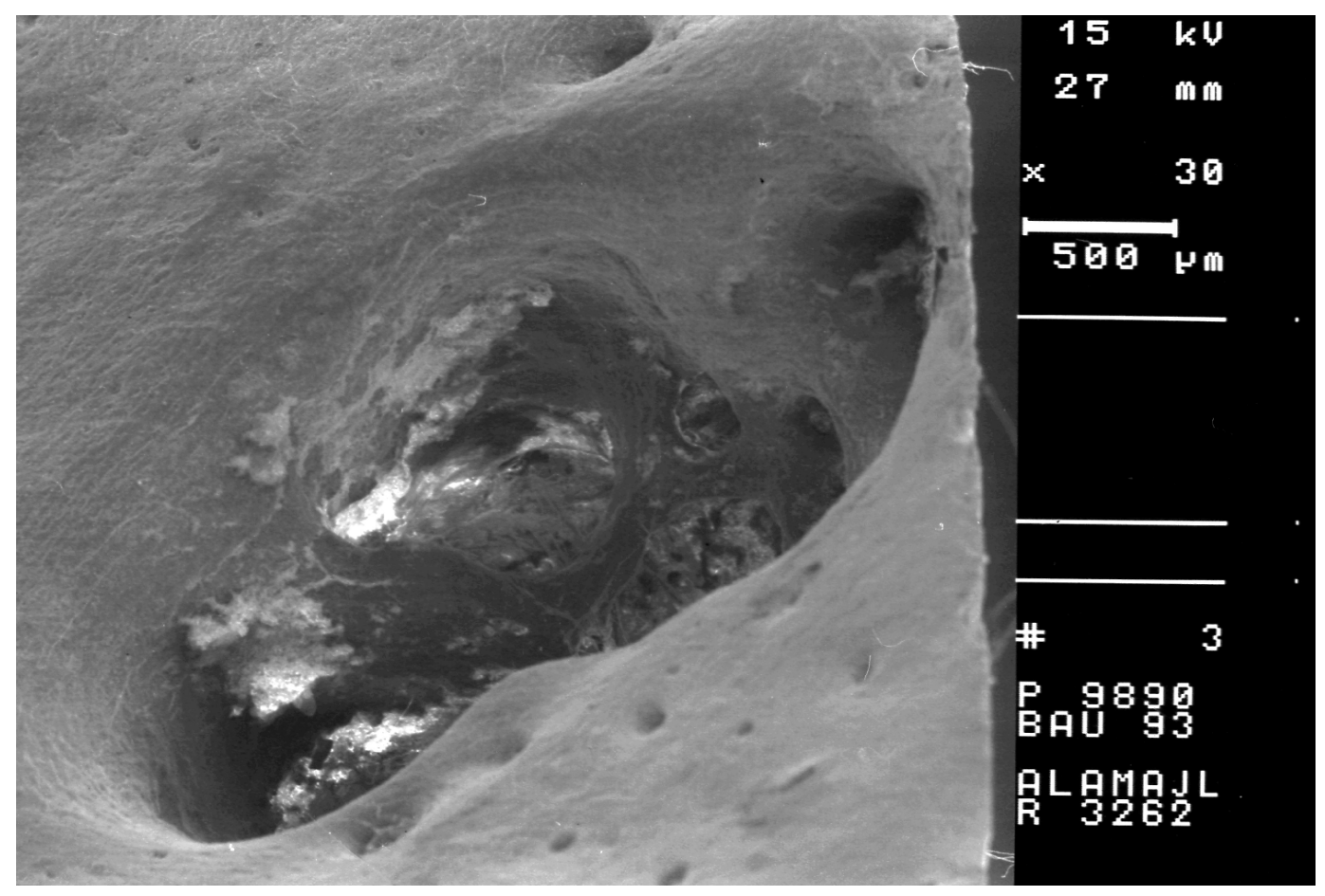

Bild 28b: Bau 93 Fossa cranii media, Ala major ossis sphenoidalis links

Rasterelektronenmikroskopische Aufnahme (REM), Vergrößerung $30 \mathrm{x}$

Gefäßforamen und einsehbare Impressionswandungen ohne postmortal erodierte Oberfläche bei intravital enstandener Läsion. 


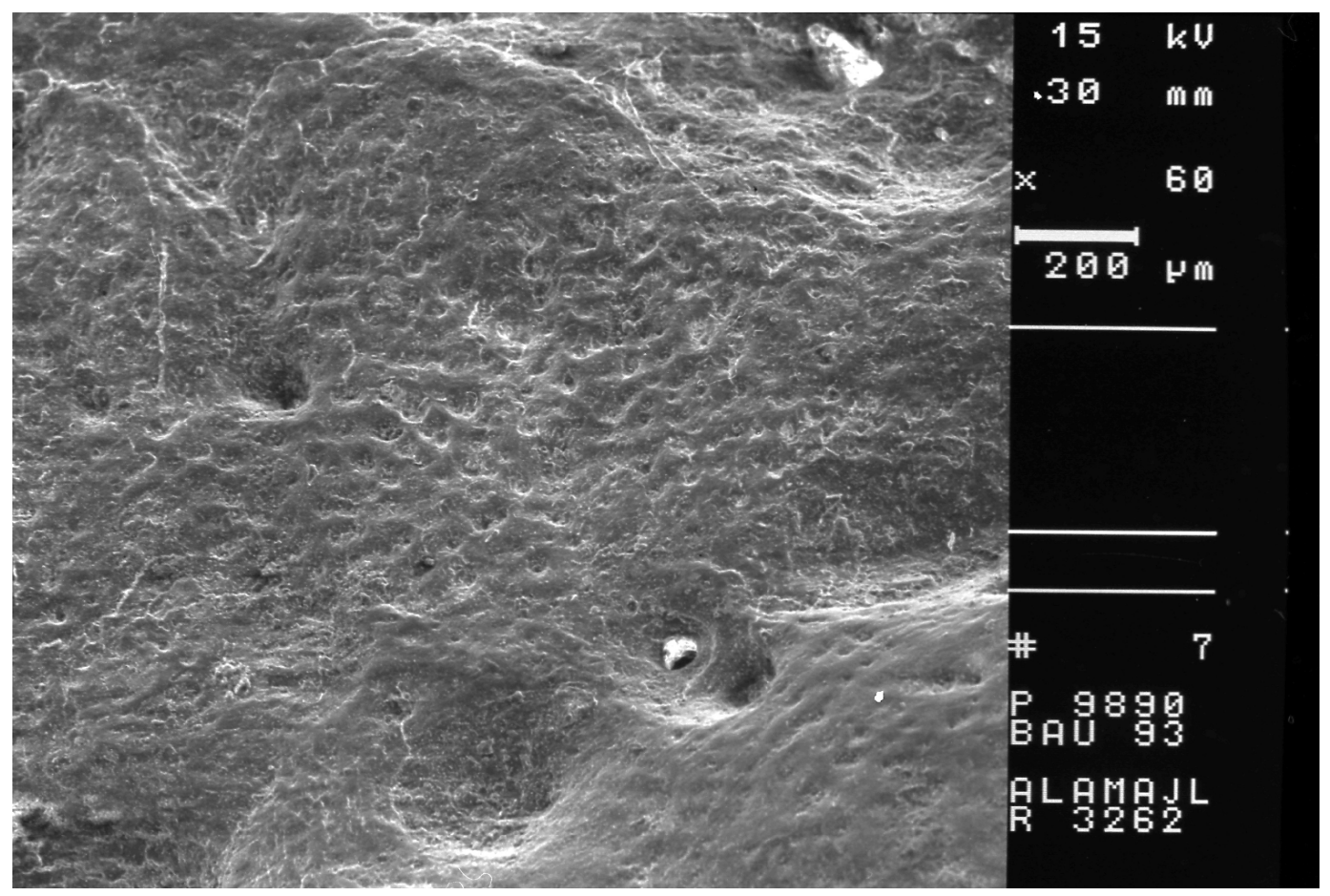

Bild 28c: Bau 93 Fossa cranii media,Ala major ossis sphenoidalis links

Rasterelektronenmikroskopische Aufnahme (REM), Vergrößerung $60 \mathrm{x}$

Tuberkelartige Gefäßimpressionen mit integrierten Auflagerungen in der Peripherie bei chronischer meningealer Reizung. 


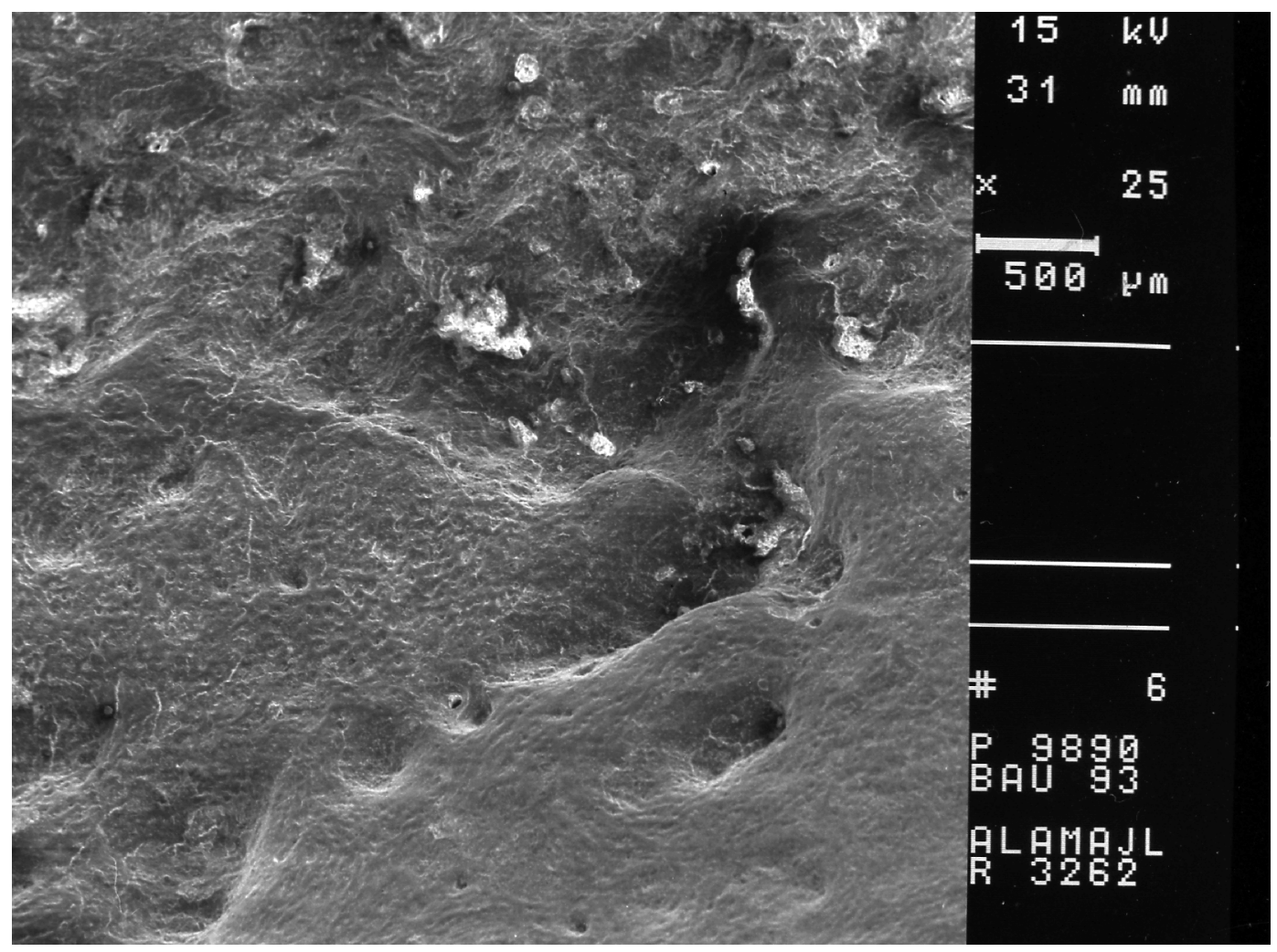

Bild 28d: Bau 93 Fossa cranii media, Ala major ossis sphenoidalis links

Rasterelektronenmikroskopische Aufnahme (REM), Vergrößerung 25 x

Läsion mit intravital veränderter Oberfläche im unteren Bildrand, postmortale Erosion in der oberen Bildhälfte. 


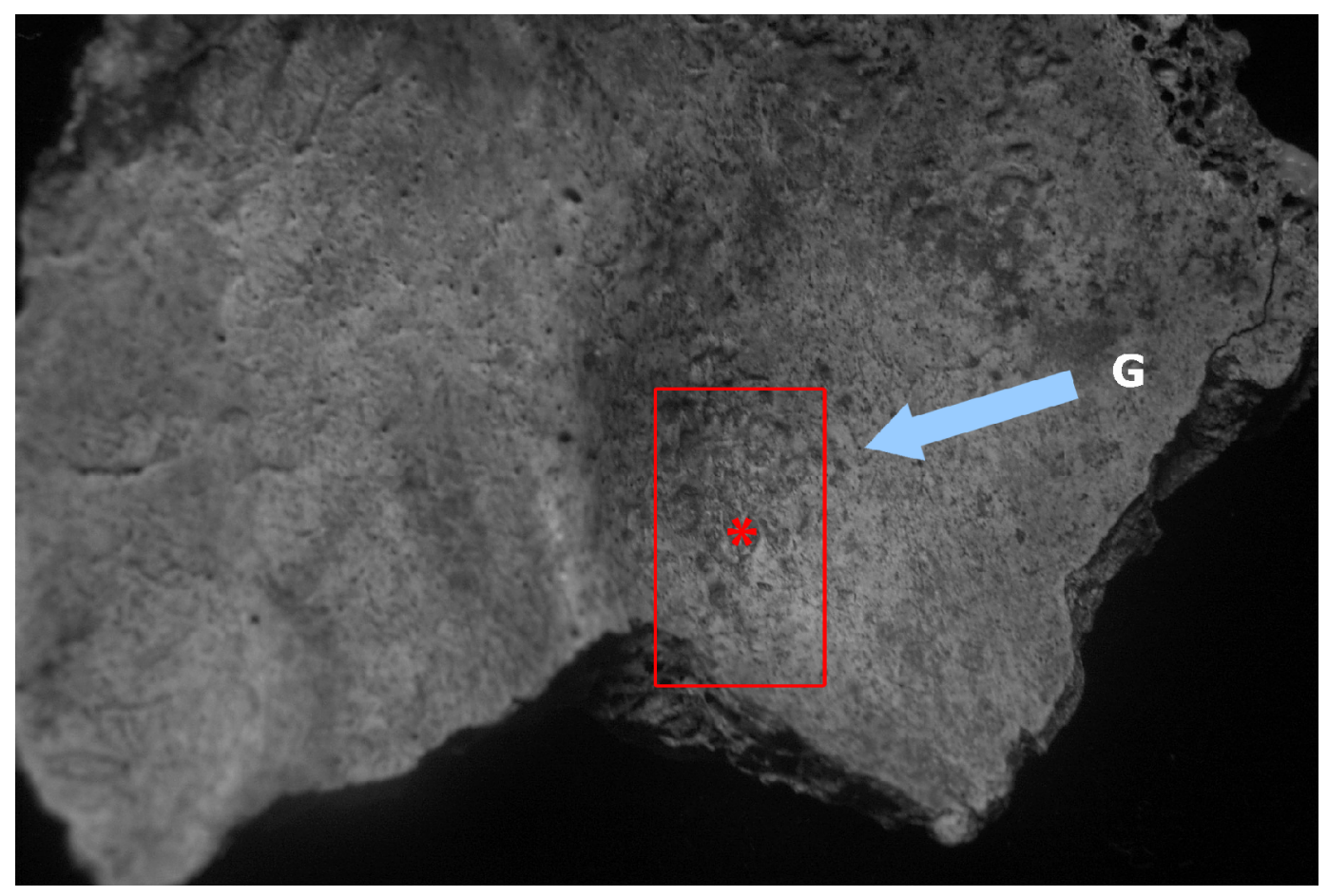

Bild 29: Bau 93, männlich = weiblich, 23-25 Jahre Os occipitale links,

\section{Fossa cranii posterior}

Konfluierende mohn- bis pfefferkorngroße Grübchen $(\mathrm{G})$ mit porösem Boden und vermehrten Gefäßimpressionen in der Peripherie.

$\rightarrow$ chronische meningeale Reizung und druckatrophische Vertiefungen

* Areal der Probenentnahme für Rasterelektronenmikroskopie, siehe Bilder 30 a-c 


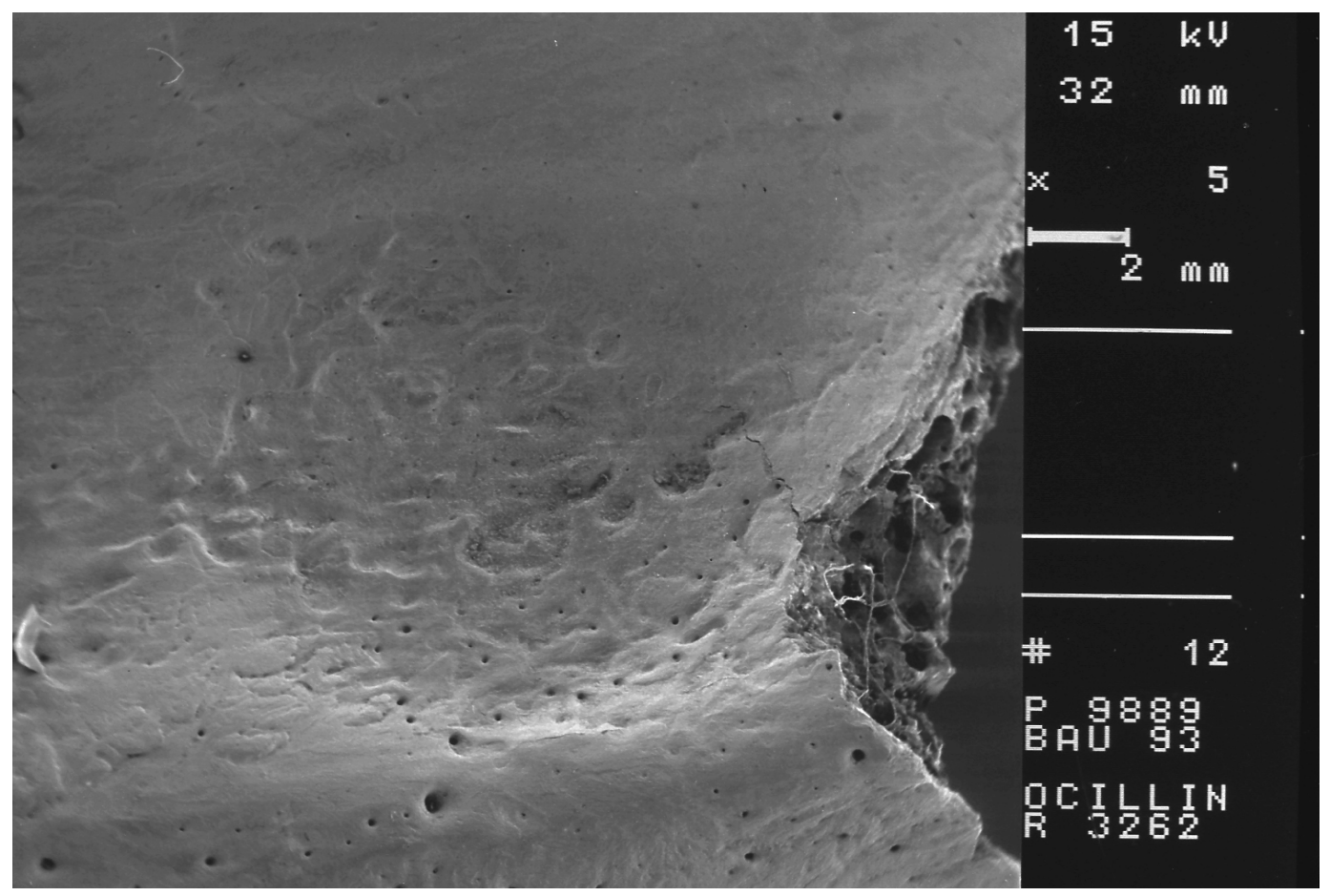

Bild 30a: Bau 93 Os occipitale links, Fossa crani posterior

Rasterelektronenmikroskopische Aufnahme (REM), Vergrößerungen $5 \mathrm{x}$

Intravitale Läsion mit integrierten Auflagerungen als Zeichen eines abgelaufenen Entzündungsprozesses.

$\rightarrow$ Verdacht auf chronischen meningealen Prozess, inaktive Leptomeningitis tuberculosa? 


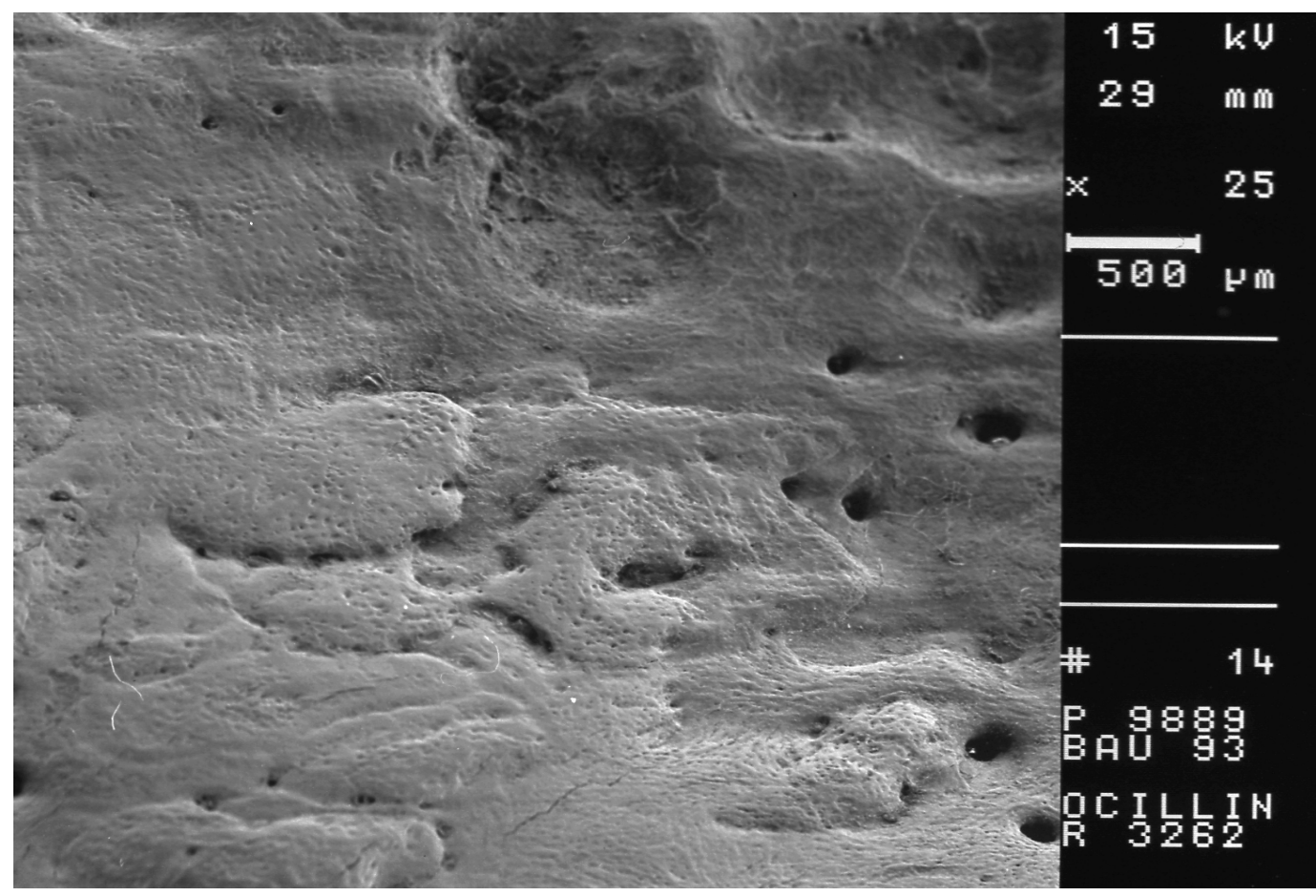

Bild 30b: Bau 93 Os occipitale links, Fossa crani posterior

Rasterelektronenmikroskopische Aufnahme (REM), Vergrößerung $25 \mathrm{x}$

Tuberkelartige Impressionen mit reizlosen Auflagerungen in der Perepherie. Auflagerungen in Organisation befindlich als Hinweis auf eine in Abheilung begriffene Läsion. 


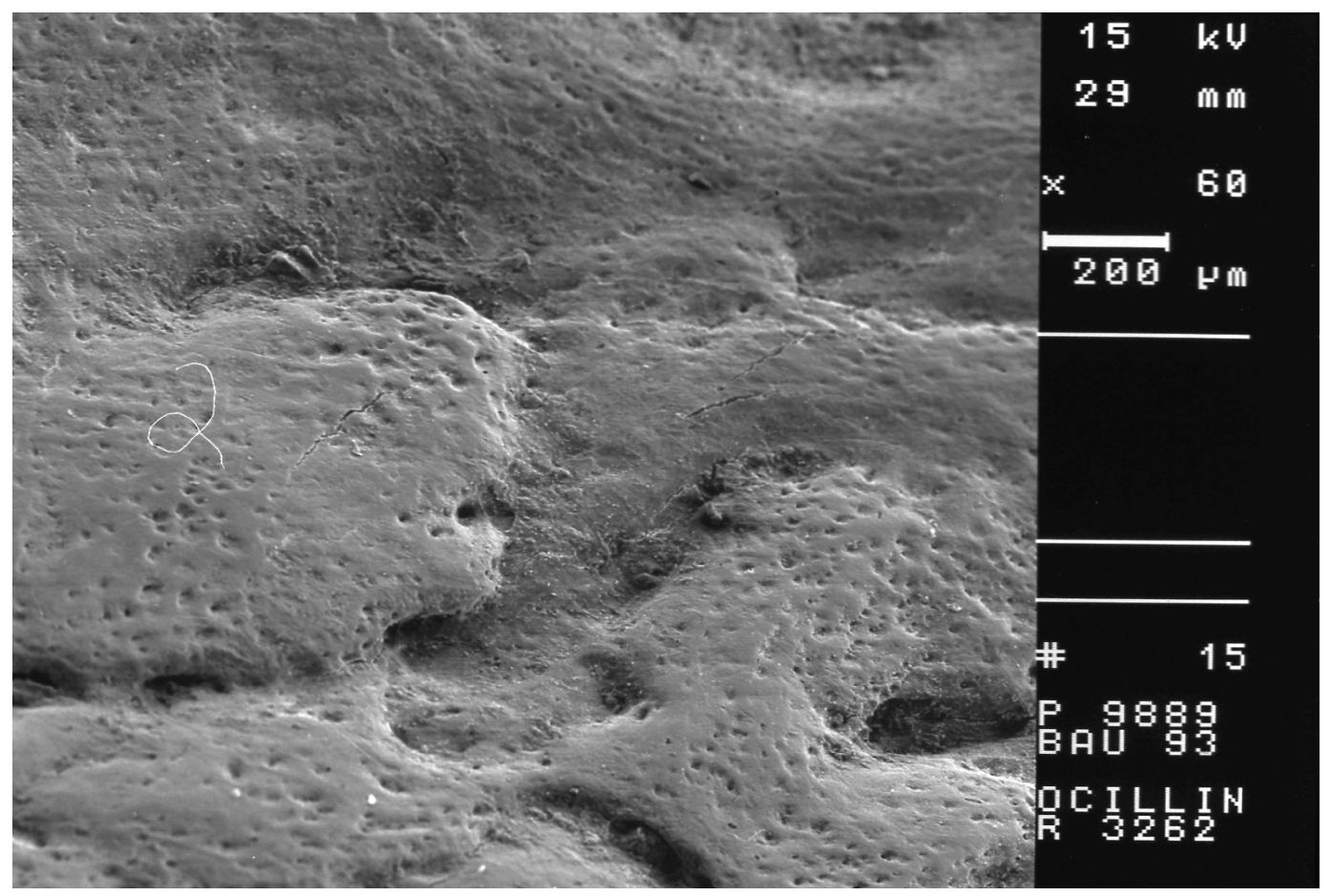

Bild 30c: Bau 93 Os occipitale links, Fossa crani posterior

Rasterelektronenmikroskopische Aufnahme (REM), Vergrößerung $60 \mathrm{x}$

Wohl mehrschichtige Auflagerungen mit reizloser Integration als Hinweis auf inaktive chronische meningeale Reizung. 


\section{Literaturverzeichnis}

Aschoff L: Pathologische Anatomie; Lehrbuch für Studierende und Ärzte. 4.Auflage, 2. Band: Spezielle pathologische Anatomie. Verlag Gustav Fischer, Jena 1919

Beck H, Steuer H: Haus und Hof in ur- und frühgeschichtlicher Zeit. Vandenhoeck \& Ruprecht Verlag, Göttingen 1997

Bergmann E v, Bruns P v, Mikulisz J v: Handbuch der praktischen Chirurgie. Fünfte umgearbeitete Auflage in 6 Bänden. 1. Band Chirurgie des Kopfes. Verlag von Ferdinand Enke, Stuttgart 1921, S. 11

Bodechtel G: Differentialdiagnose neurologischer Krankheitsbilder. Zweite, neubearbeitete und erweiterte Auflage. Georg Thieme Verlag, Stuttgart 1963, S.271; 342-343

Brandt T, Dichgans J, Diener H C: Therapie und Verlauf neurologischer Erkrankungen. Vierte, überarbeitete und erweiterte Auflage. Verlag W. Kohlhammer, Stuttgart 2003 S. 441

Braun J, Renz-Polster H: Basislehrbuch Innere Medizin. 2. Auflage, Verlag Urban \& Fischer, München, Jena 1999

Dastugue J: Die Paläopathologie. Illustrierte Geschichte der Medizin. Deutsche Ausgabe: Andreas \& Andreas. Verlagsanstalt Vaduz, 1978. Genehmigte Sonderausgabe für Karl Müller Verlag, Erlangen 1978 
Dermatologie in Praxis und Klinik. Band 2: Spezielle Dermatologie. Hrsg. v. Korting GW, Georg Thieme Verlag, Stuttgart 1980, S.18;224

Detken S: Krankhafte Veränderungen an den menschlichen Skeleten aus dem frühmittelalterlichen Reihengräberfeld Barbing-Kreuzhof. Med. Diss. Göttingen 1991

Deutsches Ärzteblatt, Jahrgang 105, Heft 7, 15. Februar 2008, S.289: Mycobacterium weltweit auf dem Vormarsch

Dollhopf KD (2002): Erwachsenenrepräsentanz oder Erosionsverlust? Gedanken zu einem Dogma der Paläodemographie des frühen Mittelalters. Germania $\underline{80}$ : 285-304

El-Najjar M, Ryan D, Turner C, Lozoff B (1976): The etiology of porotic hyperostosis among the prehistoric an historic Anasazi Indians of Southwestern United States. Am J Phys Anthropol 44:477-487

Etter HF(1982): Zur Stellung von Frau und Kind im Frühmittelalter. Eine archäologisch-anthropologische Studie. Z Schweiz Archäol Kunstgesch 39:48-57

Ferembach D, Schwidetzky I, Stloukal M (1979): Empfehlungen für die Altersund Geschlechtsdiagnose am Skelett. Homo 30:1-32

Fuchs, E: Lehrbuch der Augenheilkunde. Zwölfte vermehrte Auflage. Franz Deuticke. Leipzig und Wien 1910, S. 194-196 
Gresky J: Ätiologie und Epidemiologie der Erkrankungen des Craniums bei der Population des bajuwarischen Gräberfeldes von Harting (Oberpfalz). Ein Beitrag zur Rekonstruktion des Gesundheitsstatus frühmittelalterlicher Populationen. Med. Diss. Göttingen 2006

Gresky J, Roumelis N, Schulz C, Schultz M: Diachrone Untersuchungen zur Epidemiologie der Erkrankungen des Mittelohres in mitteleuropäischen Populationen unter geschlechtsspezifischen Aspekten. In: H Greil \& C Scheffler (eds.) Vorträge in Paläopathologie des 5. Kongress der Gesellschaft für Anthropologie (GfA) "Anthropologie der Geschlechter", 17.-20.September 2003 in Potsdam, Universitätsverlag Potsdam, Abstract 46

Gülzow M: Lehrbuch der Inneren Medizin. Infektionskrankheiten, Tuberkulose, Morbus Boeck, Erkrankungen der Verdauungsorgane, Krankheiten der Nieren, der ableitenden Harnwege und der männlichen Geschlechtsorgane, Blutkrankheiten. 2., überarbeitete Ausgabe. Hrsg. A. Sundermann, VEB Gustav Fischer Verlag, Jena 1964, S.419

Helms J, Jahrsdorfer R A: Kopf- und Hals-Chirurgie in 3 Bänden. 2., völlig neubearbeitete Auflage. Zentralherausgeber H. H. Naumann Band 2: Ohr. Herausgegeben von J. Helms, R. A. Jahrsdorfer. Georg Thieme Verlag Stuttgart New York 1996

Hensch M: Wo sie in Bunahu ihre Toten begruben - Der karolingische Ortsfriedshof von Baunach. Das Archäologische Jahr in Bayern 1998. Theiss, Stuttgart 1998

Herausgeberaufsatz (1952): Die Chemotherapie der Tuberkulose. Gesamte Inn Med Jahrgang 7 (Heft 18), S.812 
Hershkovitz I, Donoghue HD, Minnikin DE, Besra G S, Lee OY-C, et al. (2008): Detection and Molecular Characterization of 9000-Year-Old Mycobacterium tuberculosis from a Neolithic Settlement in the Eastern Mediterranean. PLoS ONE 3(10): e3426. doi:10.1371/journal.pone.0003426

Heuser H: Klinik der Zahn-, Mund- und Kieferkrankheiten. Ein Grundriß mit Berücksichtigung der Grenzgebiete. Verlag Johann Ambrosius Barth, Leipzig 1971, S. $178-181$

Huebschmann P: Pathologische Anatomie der Tuberkulose. In: Die Tuberkulose und ihre Grenzgebiete in Einzeldarstellungen. Beihefte zu den Beiträgen zur Klinik der Tuberkulose und spezifischen Tuberkuloseforschung, Band 5; hrsg. v. Ulrici H, Brauer L; Julius Springer Verlag, Berlin 1928, S. 452

Irion H: Lehrgang für Drogistenfachschulen in 4 Bänden. Band 2, 3. erweiterte Auflage. Botanik- Drogenkunde, Verlagsgesellschafft Rudolf Müller, EberswaldeBerlin-Leipzig 1942

Jürgensen T v: Lehrbuch der speziellen Pathologie und Therapie, Zweite, verbesserte und vermehrte Auflage, Verlag von Veit \& Comp., Leipzig 1889, S.119; 453460

Kastenbauer E R, Tardy jr. M E: Kopf- und Hals-Chirurgie in 3 Bänden. 2., völlig neubearbeitete Auflage. Zentralherausgeber H. H. Naumann, Band 1: Gesicht, Nase, Gesichtsschädel, Teil II Herausgegeben von E. R. Kastenbauer und M. E. Tardy jr. Georg Thieme Verlag Stuttgart New York 1995

Klose H, Grundmann G: Spezielle Chirurgie, Chirurgie des Kopfes, Walter De Gruyter \& Co., Berlin 1953, S. 204-208 
Kölbl S: Das Kinderdefizit im frühen Mittelalter - Realität oder Hypothese? Zur Deutung demographischer Strukturen in Gräberfeldern. Diss. nat. wiss. Tübingen 2004

Kollath W: Der Vollwert der Nahrung und seine Bestimmung durch Wachstum und Zellersatz. Zeitschrift für die gesamte Innere Medizin und ihre Grenzgebiete, Jahrgang 2, Heft 1/2 (1947), Arbeitsgruppe Medizinischer Verlage, Georg Thieme, Leipzig 1947 S.32

Kossel H: Vergleichende Untersuchungen über Tuberkelbazillen verschiedener Herkunft. Tuberkulose-Arbeiten aus dem kaiserlichen Gesundheitsamte. 3. Heft. Verlag von Julius Springer, Berlin 1905, S. 1-53

Kreutz K: Ätiologie und Epidemiologie von Erkrankungen des Kindesalters bei der bajuwarischen Population von Straubing (Niederbayern). (Beiträge zur Paläopathologie) Herausgegeben von M Schultz, Cuvillier Verlag, Göttingen 1997

Lang J: Praktische Anatomie. Ein Lehr- und Hilfsbuch der anatomischen Grundlagen ärztlichen Handelns. Kopf I. Band, Teil 1b Gehirn- und Augenschädel Springer Verlag Berlin, Heidelberg, New York 1979, S.440

Larsen CS: Bioarcheology. Interpreting the Behavior From the Human Skeleton. (Cambridge Studies in Biological Anthropology 21) Cambridge University Press, Cambridge 1997

Leonhardt H: Taschenlehrbuch der gesamten Anatomie, Band 3: Histologie, Zytologie und Mikroanatomie des Menschen, 8., überarbeitete und erweiterte Auflage, Georg Thieme Verlag Stuttgart, New York 1990, S.398-399 
Lewis M: Non Adult Paleopathology: Current Status and Future Potential. In: Human Osteology in Archeology an Forensic Science. Published by Cox M, Mays S. Greenwich Medical Media Ltd., London 2000, S. 40-42

Lindig W: Tuberkulose.In: Lehrbuch der Inneren Medizin, Band 1. Infektionskrankheiten, Tuberkulose, Morbus Boeck, Erkrankungen der Verdauungsorgane, Erkrankungen der Nieren, der ableitenden Harnwege und der männlichen Geschlechtskrankheiten, Blutkrankheiten. 2., überarbeitete Auflage. VEB Gustav Fischer Verlag Jena, 1964, S. 240-251

Lippert H: Lehrbuch Anatomie. 4., völlig überarbeitete Auflage. Verlag Urban \& Schwarzenberg, München, Wien, Baltimore 1996, S.456

Löffler W: Geschichte der Tuberkulose. In: Handbuch der Tuberkulose in fünf Bänden. Herausgegeben von Hein J, Kleinschmidt H, Uehlinger E. Band I: Allgemeine Grundlagen. Georg Thieme Verlag Stuttgart 1958, S. 1-24

Marx H: Kurzes Handbuch der Ohrenheilkunde. Verlag von Gustav Fischer, Jena 1939, S.10, S.229-234

Miksits H, Hahn H: Basiswissen Medizinische Mikrobiologie und Infektiologie. 2. Auflage Springer Verlag Berlin, Heidelberg, New York 1999, S. 141

Naumann HH, Scherer H: Differentialdiagnostik in der Hals-Nasen-Ohren-Heilkunde. Georg Thieme Verlag, Stuttgart, New York 1998 
Nussinovitch M, Yoeli R, Elishkevitz K, Varsano I (2004): Acute mastoiditis in children: epidemiologic, clinical, microbiologic andtherapeutic aspects over past years. Clin Pediatr (Phila) $\underline{43}(3): 261-7$

Ortner D J: Identification of Pathological Conditions in Human Skeletal Remains. $2^{\text {nd }}$ Edition. Academic Press, New York 2003

Padberg B (1992): Empirische Zugänge zu einer Epidemiologie des Mittelalters.Zeitschrift für Wissenschaftsgeschichte, $\underline{76}$ (2):164-78

Pastinszky I, Răcz I: Hautveränderungen bei Inneren Krankheiten. VEB Verlag Volk und Gesundheit, Berlin, Jena 1965, S. 546

Preyer S (2004): Die akute Mittelohrentzündung und ihre lebensbedrohlichen Komplikationen. Z Arztl., Fortbild., Qualitatssich.; $\underline{98(4): 259-263}$

Quandt J: Die zerebralen Durchblutungsstörungen des Erwachsenenalters. Ihre Grundlagen und Klinik. Zweite, völlig überarbeitete Auflage. Verlag Volk und Gesundheit, Berlin 1969, S. $778-779$

Rassner G: Dermatologie. Lehrbuch und Atlas. 6., aktualisierte Auflage. Verlag Urban \& Fischer München-Jena 2000, S. 73-75; $92-93$

Redecker F: Epidemiologie und Statistik der Tuberkulose. In: Handbuch der Tuberkulose in fünf Bänden. Herausgegeben von Hein $J$, Kleinschmidt $H$, Uehlinger E. Band I: Allgemeine Grundlagen. Georg Thieme Verlag Stuttgart 1958, S. 413 
Roberts C A, Buikstra J E: The Bioarchaeology Of Tuberculosis. A Global Viev on a Reemerging Disease. University Press of Florida, Gainesville 2003, S. 262263

Roumelis N: The Palaeopathology of Kirchberg. Evidence of Deficiency, Inflammatory and Tumorous Disease in a Medieval Rural Population in Hessia, Germany. Doctoral Thesis in Osteoarchaeolgy at Stockholm Uniersity, Sweden 2007 S. 140, S. $142-145$

Rüster D: Alte Chirurgie-Legende und Wirklichkeit. 1. Auflage, Verlag Volk und Gesundheit, Berlin 1984, S. 9-31

Schlomm T: Spuren pathologischer Prozesse an den menschlichen Schädeln aus dem frühmittelalterlichen Reihengräberfeld Barbing-Kreuzhof unter besonderer Berücksichtigung der unspezifischen und tuberkulösen Entzündungen im Bereich der Meningen und der pneumatischen Schädelräume. Med. Diss. Göttingen 2000

Schuermann H: Krankheiten der Mundschleimhaut und der Lippen. Verlag Urban und Schwarzenberg, München und Berlin 1958, S. 545

Schultz M: Umwelt und Krankheit des vor- und frühgeschichtlichen Menschen. In: Kindlers Enzyklopädie; herausgegeben von Loakker N, Wendt H; Kindler-Verlag, München 1982

Schultz M: Die mikroskopische Untersuchung prähistorischer Skeletfunde. Anwendung und Aussagemöglichkeiten der differentialdiagnostischen Untersuchung in der Paläopathologie. Tagungsberichte zum Paläopathologischen Sympo- 
sium in Liestal (Baselland) 21.-23. Juni 1984, Band 1, hrsg. vom Amt der Museen und Archäologie BL, Liestal und dem Anthropologischen Forschungsinstitut Aesch; Aesch 1986

Schultz, M : Methoden der Licht- und Elektronenmikroskopie. In: Knussmann, R. (ed.): Anthropologie. Handbuch der vergleichenden Biologie des Menschen 1, 1. G. Fischer Verlag, Stuttgart 1988, S. $698-730$

Schultz, M : Paläopathologische Diagnostik. In: Knussmann, R. (ed.): Anthropologie: Handbuch der vergleichenden Biologie des Menschen. Band I, 1 Wesen und Methoden der Anthropologie. G. Fischer Verlag, Stuttgart 1988, S. 480-496

Schultz M: Spuren unspezifischer Entzündungen an prähistorischen und historischen Schädeln. Ein Beitrag zur Paläopathologie. Med. Habilitationsschrift Georg-August-Universität zu Göttingen, Göttingen 1987

Schultz M (1989): Ergebnisse osteologischer Untersuchungen an mittelalterlichen Kinderskeleten unter besonderer Berücksichtigung anatolischer Populationen. Anthropol Anz 47:39-50

Schultz M: Spuren unspezifischer Entzündungen an prähistorischen und historischen Schädeln. Ein Beitrag zur Paläopathologie. In: Kufmann B (ed.), Anthropologische Beiträge 4A und 4B. Aesch und Basel: Anthropologischen Forschungsinstitut Aesch und Anthropologische Gesellschaft Basel 1993, S. 1-84

Schultz M: Ergebnisse mikroskopischer Untersuchungen an archäologischen Skeletfunden. Ein Beitrag zur Paläopathologie. Beiträge zur Archäozoologie und prähistorischen Anthropologie. In: Kokabi M, Wahl J, editors. Forschungen und Berichte zur Vor- und Frühgeschichte in Baden-Württemberg 1994 53:107-116 
Schultz M: Microscopic structure of bone. In: Haglund WD, Sorg MH, editors. Forensic taphonomy. The postmortem fate of human remains. CRC Press LCC Boca Raton 1997, S. 187-199; 201-204

Schultz M : The role of tuberculosis in infancy and childhood in prehistoric and historic populations. In Gy Palfî, O Dutour, J Deák and I Hutás (eds): Tuberculosis past and present. Szeged: Golden Book Publisher Ltd. And Tuberculosis Foundation 1999, S. 501-507

Schultz M (2001): Paleohistopathology of Bone: A New Approach to the Study of Ancient Diseases. Yearbook Phys Anthropol 44:106-147

Schultz M: Light microscopic analysis in skeletal paleopathology. In: Ortner DJ (ed): Identification of pathological conditions in human skeletal remains. Elsevier Science Amsterdam, Boston, London 2003, S. 73-108

Schultz M, Drommer R: Möglichkeiten der Präparateherstellung aus dem Gesichtsschädelbereich für die Makroskopische und mikroskopische Untersuchung unter Verwendung neuer Kunststofftechniken. In: Hoppe W (ed.): Experimentelle Mund-Kiefer-Gesichtschirurgie: Mikroskopische Eingriffe. Thieme Verlag, Stuttgart 1983, S. 95-97

Schultz M, Larsen CS, Kreutz K: Disease in Spanish Florida. Microscopy of Porotic Hyperostosis and Cribra Orbitalia. In: Larsen CS (ed.) Bioarchaeology of Spanish Florida: The Impact of Colonialism. University Press of Florida, Gainesville 2001, S.207-225 
Seifert G: Doerr, Seifert, Uelinger: Spezielle pathologische Anatomie, Oralpathologie 3, Mundhöhle, angrenzendes Weichteil- und Knochengewebe, Band 1/III, 2. Auflage. Springer-Verlag Berlin, Heidelberg, New York, Barcelona, Budapest, Hongkong, London, Mailand, Paris, Singapur, Tokio 2000, S.217

Simon C, Stille W: Antibiotikatherapie in Klinik und Praxis. 10. Auflage, Schattauer Verlag, Stuttgart, New York 2000, S. 423

Sjvøld T: Geschlechtsdiagnose am Skelett; in: Handbuch der vergleichenden Biologie des Menschen, Band 1; hrsg. von Knussmann R; Fischer Verlag, Stuttgart $1988,444-479$

Sobotta J: Atlas der Anatomie des Menschen. 20. Auflage. Herausgegeben von Putz R und Pabst R; Band 1 Kopf, Hals, obere Extremität. Urban \& Schwarzenberg, München 1993

Steinberg C: Lehrbuch der Allgemeinen und der speziellen Pathologischen Anatomie. 2. Auflage, Verlag von F.C.W. Vogel, Berlin 1933

Steffenhagen K (1908): Über die Beziehungen der menschlichen Tuberkulose zu denen der Perlsucht des Rindes. Zusammenfassende Übersicht auf Grund der Tuberkulosearbeiten aus dem Kaiserlichen Gesundheitsamt. Aus dem Kaiserlichen Gesundheitsamt. Berl Klin Wochenschr, 33:1-8

Stochdorph O: Nervensystem. In: Lehrbuch der Allgemeinen Pathologie und der Pathologischen Anatomie. 33. Auflage, hrsg. v. Eder M, Gedigk P. Springer Verlag, Berlin 1990 S. 501-534 
Szilvássy J: Altersdiagnose am Skelett, in: Handbuch der vergleichenden Biologie des Menschen, Band 1; hrsg. von Knussmann R, Fischer Verlag, Stuttgart 1988, $421-443$

Taute M: Weitere Untersuchungen über Tuberkelbazillen verschiedener Herkunft mit besonderer Berücksichtigung der primären Darm- und Mesenterialtuberkulose. Tuberkulose-Arbeiten aus dem Kaiserlichen Gesundheitsamte. 7. Heft. Verlag von Julius Springer. Berlin 1907 S. 15-76

Templin O: Die Kinderskelete von Bettingen im Kanton Basel-Stadt (Schweiz). Eine palaäopathologische Untersuchung. Med. Diss. Göttingen 1993

Ulrici H: Klinik der Lungentuberkulose. Dritte, verbesserte Auflage der Diagnostik und Therapie der Lungen- und Kehlkopftuberkulose. Springer-Verlag, Berlin 1944 S. 5, S. 49

Unsöld R, Greeven G: Entzündliche Orbitaerkrankungen. Klinik, Radiologie, Differentialdiagnose. Springer-Verlag, Berlin, Heidelberg 1997 S. 109-114

Vincent V, Gutierrez Perez MC: The Agent of Tubeculosis. In: Palfi G, Dutou O, Deak J and Hutas I (eds.), Tuberculosis: Past and present. Golden Book Publishers and Tuberculosis Foundation, Budapest Szeget 1999, S. 139-143

Walker P (2009): The Causes of Porotic Hyperostosis and Cribra Orbitalia: A Reappraisal of the Iron-Deficiency-Anaemia Hypothesis. Am J Phys Anthropol 139: $109-125$ 
Walter E: 1200-jähriges Baunach. Heimatbeilage zum Oberfränkischen Schulanzeiger. Bayreuth, Oktober 2002 Nr. 297

Wapler U (2004): Is Cribra Orbitalia synonymous with anemia? Analysis and interpretation of cranial pathology in Sudan. Am J Phys Anthropol 123:333-339

Weber A: Untersuchungen über das Vorkommen von Tuberkelbazillen in Drüsen und Tonsillen von Kindern, welche sich bei der Obduktion als frei von Tuberkulose erwiesen hatten. Tuberkulose-Arbeiten aus dem kaiserlichen Gesundheitsamte. 7. Heft. Verlag von Julius Springer, Berlin 1907 S.102-105

Wells C: Diagnose 5000 Jahre später. Krankheit und Heilkunst in der Frühzeit des Menschen. Gustav Lübbe Verlag, Bergisch-Gladbach 1967, S.13-20

Werner JA, Lippert BM: HNO-Heilkunde; Farbatlas zur Befunderhebung, Differentialdiagnostik und Therapie. Schattauer Verlag, Stuttgart, New York 2003

Wittmaack K: Über die normale und pathologische Pneumatisation des Schläfenbeines. Gustav Fischer, Jena 1918

Younis RT (2002): Intracranial complications of sinusitis: a 15-year review of 39 cases. Ear Nose Throat Journal 81(9): 636-44

Ziegler E: Lehrbuch der allgemeinen Pathologie und der pathologischen Anatomie in zwei Bänden, neunte neu bearbeitete Auflage, Erster Band Allgemeine Pathologie, Verlag von Gustav Fischer, Jena 1898 a, S.684 
Ziegler E: Lehrbuch der allgemeinen Pathologie und der pathologischen Anatomie in zwei Bänden, neunte neu bearbeitete Auflage, Zweiter Band specielle pathologische Anatomie. Verlag von Gustav Fischer, Jena 1898 b, S. 624-628 


\section{Abbildungsverzeichnis}

Abbildung 1: Formblatt zur pathologischen Auswertung des Cranium.................10

Abbildung 2: Geschlechtsverteilung in der gesamten Population von

Baunach (7. - 9. Jhd.).......................................................... 13

Abbildung 3: Geschlechtsverteilung der Baunacher Individuen mit erhaltenem

Schädel (7. - 9. Jhd.)................................................................ 13

Abbildung 4: Sterbehäufigkeit der Individuen mit Schädel (7. - 9. Jhd.)..............14

Abbildung 5: Absterbekurve der Individuen mit Schädel (7. - 9. Jhd.)................15

Abbildung 6: Sterbewahrscheinlichkeit der Individuen mit Schädel

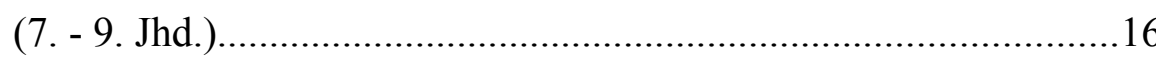

Abbildung 7: Relativer Anteil der gestorbenen Männer (7. - 9. Jhd.)....................17

Abbildung 8: Absterbekurve der Männer (7. - 9. Jhd.)........................................ 17

Abbildung 9: Sterbewahrscheinlichkeit der Männer (7. - 9. Jhd.)........................18

Abbildung 10: Realtiver Anteil der gestorbenen Frauen (7. - 9. Jhd.)..................19

Abbildung 11: Absterbekurve der Frauen (7. - 9. Jhd.).........................................19

Abbildung 12: Sterbewahrscheinlichkeit der Frauen (7. - 9. Jhd.).......................20

Abbildung 13: Relativer Anteil der unbestimmbaren Individuen (7. - 9. Jhd.) ..21

Abbildung 14: Absterbekurve der unbestimmbaren Individuen (7. - 9. Jhd.) .......22

Abbildung 15: Sterbewahrscheinlichkeit der unbestimmbaren Individuen

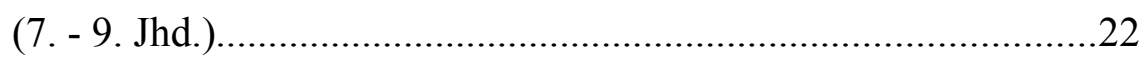

Abbildung 16: Begünstigende Faktoren und Wechselwirkungen eines

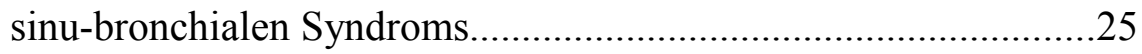

Abbildung 17: Geschlechtsverteilung der Nasenhöhlen....................................27

Abbildung 18: Geschlechtsspezifische Verteilung der

Nasenhöhlenentzündungen.

Abbildung 19: Geschlechtsspezifische Erkrankungshäufigkeit der

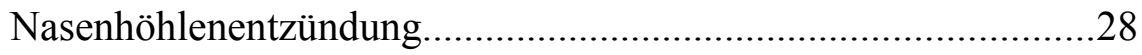

Abbildung 20: Übersicht topographische Beziehung der Nasenhöhle .................30 
Abbildung 21: Beziehungen des Zahnhalteapparates des Oberkiefers zur

Kieferhöhle beim Erwachsenen 32

Abbildung 22: Mögliche Richtungen der Infektionsausbreitung bei Sinusitis......34

Abbildung 23: Geschlechtsverteilung der auswertbaren Kieferhöhlen. 35

Abbildung 24: Geschlechtsspezifische Verteilung der

Kieferhöhlenentzündungen.

Abbildung 25: Einteilung nach Schweregrad der Kieferhöhlenentzündung... 38

Abbildung 26: Endocranielle Komplikationsmöglichkeiten bei

Sinusitis frontalis

Abbildung 27: Geschlechtsverteilung der Individuen mit auswertbaren

Stirnhöhlen.

Abbildung 28: geschlechtsspezifische Verteilung der Stirnhöhlenentzündung.....40

Abbildung 29: Einteilung nach Schweregrad der Stirnhöhlenentzündung.

Abbildung 30: Prozentuale Schweregradeinteilung bei Stirnhöhlenentzündung...42

Abbildung 31: Schematische Darstellung möglicher Infektionswege einer

Otitis media

Abbildung 32: Mögliche intracranielle Ausbreitungsrichtungen bei

Otitis media. .46

Abbildung 33: Geschlechtsverteilung der Mittelohren .47

Abbildung 34: Geschlechtsspezifische Verteilung der Mittelohrentzündung........48

Abbildung 35: Prozentuale Verteilung der Mittelohrentzündung. .48

Abbildung 36: Mögliche Abszessentlastungsrichtungen bei Mastoiditis. 50

Abbildung 37: Geschlechtsverteilung der auswertbaren Mastoide .52

Abbildung 38: Geschlechtsspezifische Verteilung der

Warzenfortsatzentzündung..... .53

Abbildung 39: Prozentuale Verteilung der Warzenfortsatzentzündung. .53

Abbildung 40: Pneumatisationsart des Warzenfortsatzes.

Abbildung 41: Beziehungen zwischen der Erkrankung des Mittelohres und des Mastoids. 
Abbildung 42: Zusammenhang zwischen Mittelohrentzündung und

Kieferhöhlenentzündung.

Abbildung 43: Schematisierter Frontalschnitt durch Schädeldach und

Hirnhäute am Ansatz der Falx cerebri 59

Abbildung 44: Geschlechtsverteilung der Individuen mit Schädelgruben.

Abbildung 45: Geschlechtsspezifische Verteilung der tuberkulösen Meningitis...64

Abbildung 46: Geschlechtsdifferenzierte prozentuale Verteilung der tuberkulösen Meningitis.

Abbildung 47: Verteilung der Fälle mit Spuren meningealer Reaktionen

in den Schädelgruben .66

Abbildung 48: Prozentuale Verteilung der meningealen Reaktionen. 68

Abbildung 49: Geschlechtsspezifische Verteilung der Individuen mit

Schädeldach.

Abbildung 50: Geschlechtsspezifische Verteilung

Kopfschwartenentzündung Lamina externa.. 72

Abbildung 51: Läsionen im Bereich der Lamina externa differenziert

nach Geschlecht.

Abbildung 52: Geschlechtsverteilung der Individuen mit

Sinus sagittalis superior

Abbildung 53: Geschlechtsspezifische Verteilung der erkrankten

Sinus sagittalis superiores.

Abbildung 54: Geschlechtsspezifische Verteilung der betroffenen Regionen des erkrankten Sinus sagittalis superior.

Abbildung 55: Geschlechtsverteilung der Individuen mit Sinus transversus.

Abbildung 56: Geschlechtsspezifische Verteilung der erkrankten

Sinus transversi.

Abbildung 57: Zusammenhang zwischen erkranktem Sinus transversus und

Mastoiditis.

Abbildung 58: Verteilung der möglichen Paarbildungen bei Sinus transversus

und Mastoid. 
Abbildung 59: Geschlechtsverteilung der Individuen mit Sinus sigmoideus........86

Abbildung 60: Geschlechtsspezifische Verteilung der erkrankten

Sinus sigmoidei.. .86

Abbildung 61: Zusammenhang zwischen Erkrankungen des

Sinus sigmoideus und des Mittelohrs

Abbildung 62: Verteilung der möglichen Paarbildungen bei

Sinus sigmoideus und Mittelohr . .88

Abbildung 63: Geschlechtsverteilung der Individuen mit Orbita. .92

Abbildung 64: Geschlechtsspezifische Verteilung der erkrankten Orbitae..

Abbildung 65: Geschlechtsspezifische Verteilung stark ausgeprägter

Veränderungen am Orbitadach.

Abbildung 66: Zusammenhang zwischen Erkrankungen der Orbita und endocraniellen Infektionen. . .95

Abbildung 67: Geschlechtsverteilung der Individuen mit hartem Gaumen . .98

Abbildung 68: Geschlechtsspezifische Verteilung der Entzündungen des harten Gaumens

Abbildung 69: Geschlechtsdifferenzierte prozentuale Verteilung der

Entzündungen des harten Gaumens .99

Abbildung 70: Geschlechtsspezifische Häufigkeit des Torus palatinus. 100 


\section{Tabellenverzeichnis}

Tabelle 1: Lebensalter Klasseneinteilung.......................................................6

Tabelle 2: Individuen Baunach mit Cranium....................................................... 9

Tabelle 3: Komplikationen der Otitis media.......................................................45

Tabelle 4: Ursachen und klinische Beispiele einer Sinusvenenthrombose (SVT). 77 


\section{Danksagung}

Bedanken möchte ich mich in erster Linie bei meinem Doktorvater Prof. Dr. med.

Dr. phil. Michael Schultz, Leiter der Arbeitsgruppe Paläopathologie im Zentrum Anatomie der Universität Göttingen, für das Bereitstellen des Materials sowie die wissenschaftliche Ausbildung und Betreuung.

Danken möchte ich auch der gesamten Arbeitsgruppe Paläopathologie in Göttingen, insbesondere Herrn Dr. N. Roumelis, PhD sowie Herrn RNDr. J. Nowacek, für anregende Gespräche und die freundschaftliche Zusammenarbeit bei der Bearbeitung des Materials im Labor und auf paläopathologischen Kampagnen.

Ebenso gilt mein Dank Fr. I. Hettwer-Steeger und Herrn M. Brandt für die Fertigstellung der mikroskopischen Präparate.Weiterhin danke ich Dr. med. J. Henne und Dr. med. L. Jonetz-Mentzel für das Korrekturlesen sowie kritische Anmerkungen. 


\section{Lebenslauf}

Am 8.10.1978 wurde ich als erstes Kind der Bauingeneurin Christine Schulz und des Elektrikermeisters Rüdiger Schulz in Sondershausen geboren.

Eingeschult wurde ich 1985 in der 21. POS in Dessau. Die weitere Grundschulzeit verbrachte ich von 1986 bis 1991 an der POS „Anton Saefkow“ in Sondershausen. Die Schulzeit schloss ich 1997 am „Geschwister Scholl“ Gymnasium in Sondershausen ab, welches ich von 1991 an besuchte.

Anschließend leistete ich meinen Zivildienst am Kyffhäuser Kreiskrankenhaus in Sondershausen.

1998 begann ich das Medizinstudium an der Georg-August-Universität Göttingen. Dort legte ich mein Physikum im Jahr 2000 ab. Nach dem ersten Staatsexamen im Jahr 2001 begann ich meine Promotion bei Herrn Prof. Dr. med. Dr. phil. M. Schultz im Zentrum Anatomie. Dort arbeitete ich von 2002 bis 2003 als wissenschaftlicher Mitarbeiter in der Arbeitsgruppe Paläopathologie. Von 2003 bis 2004 war ich im Katholischen Krankenhaus in Weende auf der internistischen Intensivstation als studentische Pflegehilfskraft angestellt.

Im Rahmen meiner paläopathologischen Dissertation arbeitete ich 2003/04 bei mehreren Grabungskampagnen, unter anderem in der Slowakei und in der Türkei. Nach abgeschlossenem zweiten Staatsexamen im Jahr 2004 wechselte ich an die Friedrich-Schiller-Universität Jena.

Nach bestandenem dritten Staatsexamen und Erlangen der Approbation im Jahr 2005 befinde ich mich in der Ausbildung zum Allgemein- und Viszeralchirurgen im Helios Kreiskrankenhaus Gotha. 